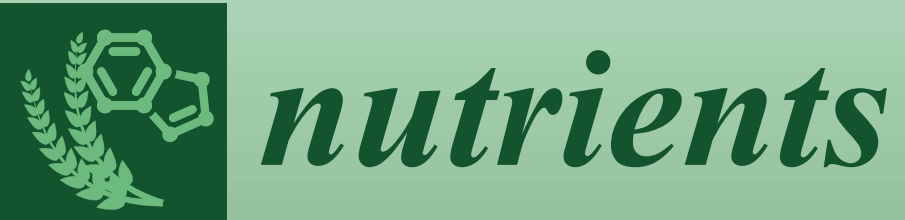

\title{
Nutritional Intake and the Risk for Non-Alcoholic Fatty Liver Disease (NAFLD)
}

Edited by Ina Bergheim and Jörn M. Schattenberg Printed Edition of the Special Issue Published in Nutrients 
Nutritional Intake and the Risk for Non-Alcoholic Fatty Liver Disease (NAFLD) 



\section{Nutritional Intake and the Risk for Non-Alcoholic Fatty Liver Disease (NAFLD)}

Special Issue Editors

Ina Bergheim

Jörn M. Schattenberg 
Ina Bergheim

University of Vienna

Austria
Jörn M. Schattenberg

University Medical Center of the

Johannes Gutenberg University

Germany

Editorial Office

MDPI

St. Alban-Anlage 66

4052 Basel, Switzerland

This is a reprint of articles from the Special Issue published online in the open access journal Nutrients (ISSN 2072-6643) in 2018 (available at: https://www.mdpi.com/journal/nutrients/special_issues / Fatty_Liver_Disease)

For citation purposes, cite each article independently as indicated on the article page online and as indicated below:

LastName, A.A.; LastName, B.B.; LastName, C.C. Article Title. Journal Name Year, Article Number, Page Range.

ISBN 978-3-03897-598-4 (Pbk)

ISBN 978-3-03897-599-1 (PDF)

(C) 2019 by the authors. Articles in this book are Open Access and distributed under the Creative Commons Attribution (CC BY) license, which allows users to download, copy and build upon published articles, as long as the author and publisher are properly credited, which ensures maximum dissemination and a wider impact of our publications.

The book as a whole is distributed by MDPI under the terms and conditions of the Creative Commons license CC BY-NC-ND. 


\section{Contents}

About the Special Issue Editors $\ldots \ldots \ldots \ldots \ldots \ldots \ldots$ vii

Preface to "Nutritional Intake and the Risk for Non-Alcoholic Fatty Liver Disease (NAFLD)" ix

Hiroyuki Niwa, Katsumi Iizuka, Takehiro Kato, Wudelehu Wu, Hiromi Tsuchida, Ken Takao, Yukio Horikawa and Jun Takeda

ChREBP Rather Than SHP Regulates Hepatic VLDL Secretion

Reprinted from: Nutrients 2018, 10, 321, doi:10.3390/nu10030321 . . . . . . . . . . . . . . 1

Mahmud Mahamid, Naim Mahroum, Nicola Luigi Bragazzi, Kasem Shalaata, Yarden Yavne, Mohammad Adawi, Howard Amital and Abdulla Watad

Folate and B12 Levels Correlate with Histological Severity in NASH Patients

Reprinted from: Nutrients 2018, 10, 440, doi:10.3390/nu10040440 . . . . . . . . . . . . . . . 13

Shilpa Tiwari-Heckler, Hongying Gan-Schreier, Wolfgang Stremmel, Walee Chamulitrat and Anita Pathil

Circulating Phospholipid Patterns in NAFLD Patients Associated with a Combination of Metabolic Risk Factors

Reprinted from: Nutrients 2018, 10, 649, doi:10.3390/nu10050649 . . . . . . . . . . . . . . .

Daisuke Uchida, Akinobu Takaki, Takuya Adachi and Hiroyuki Okada

Beneficial and Paradoxical Roles of Anti-Oxidative Nutritional Support for Non-Alcoholic Fatty Liver Disease

Reprinted from: Nutrients 2018, 10, 977, doi:10.3390/nu10080977 . . . . . . . . . . . . . . .

Derek Tobin, Merethe Brevik-Andersen, Yan Qin, Jacqueline K. Innes and Philip C. Calder Evaluation of a High Concentrate Omega-3 for Correcting the Omega-3 Fatty Acid Nutritional Deficiency in Non-Alcoholic Fatty Liver Disease (CONDIN)

Reprinted from: Nutrients 2018, 10, 1126, doi:10.3390/nu10081126 . . . . . . . . . . . . . . . .

Jean-Pascal De Bandt, Prasanthi Jegatheesan and Naouel Tennoune-El-Hafaia

Muscle Loss in Chronic Liver Diseases: The Example of Nonalcoholic Liver Disease

Reprinted from: Nutrients 2018, 10, 1195, doi:10.3390/nu10091195 . . . . . . . . . . . . . .

Andras Franko, Dietrich Merkel, Marketa Kovarova, Miriam Hoene, Benjamin A. Jaghutriz, Martin Heni, Alfred Königsrainer, Cyrus Papan, Stefan Lehr, Hans-Ulrich Häring and Andreas Peter

Dissociation of Fatty Liver and Insulin Resistance in I148M PNPLA3 Carriers: Differences in Diacylglycerol (DAG) FA18:1 Lipid Species as a Possible Explanation

Reprinted from: Nutrients 2018, 10, 1314, doi:10.3390/nu10091314 . . . . . . . . . . . . . . . .

Janin Henkel, Eugenia Alfine, Juliana Saín, Korinna Jöhrens, Daniela Weber, José P. Castro, Jeannette König, Christin Stuhlmann, Madita Vahrenbrink, Wenke Jonas, André Kleinridders and Gerhard P. Püschel Soybean Oil-Derived Poly-Unsaturated Fatty Acids Enhance Liver Damage in NAFLD Induced by Dietary Cholesterol

Reprinted from: Nutrients 2018, 10, 1326, doi:10.3390/nu10091326 . . . . . . . . . . . . . 86

Anika Nier, Annette Brandt, Ina Barbara Conzelmann, Yelda Özel and Ina Bergheim

Non-Alcoholic Fatty Liver Disease in Overweight Children: Role of Fructose Intake and Dietary Pattern

Reprinted from: Nutrients 2018, 10, 1329, doi:10.3390/nu10091329 . . . . . . . . . . . . . 103 
Marcin Krawczyk, Dominika Maciejewska, Karina Ryterska, Maja Czerwińka-Rogowska, Dominika Jamioł-Milc, Karolina Skonieczna-Żydecka, Piotr Milkiewicz,

Joanna Raszeja-Wyszomirska and Ewa Stachowska

Gut Permeability Might be Improved by Dietary Fiber in Individuals with Nonalcoholic Fatty

Liver Disease (NAFLD) Undergoing Weight Reduction

Reprinted from: Nutrients 2018, 10, 1793, doi:10.3390/nu10111793 . . . . . . . . . . . . . . . . . 121 


\section{About the Special Issue Editors}

Ina Bergheim, PhD, is a nutrition scientist with a strong background in nutritional medicine. After obtaining a PhD at the University of Hohenheim, Stuttgart, Germany, she pursued her scientific career as a PostDoc at the Robert-Bosch-Hospital, Stuttgart, Germany, working on the molecular mechanisms underlying in the development of gallstone disease in humans, followed by a PostDoc at the University of Louisville, Kentucky, USA. Ever since she has focused her research on the molecular mechanisms involved in the development of metabolic liver diseases and the nutrition-based prevention and therapy of these diseases. In 2012 she was appointed as full professor, first at the Friedrich-Schiller-University, Jena, Germany, and since 2016 at University of Vienna, Vienna, Austria, Department of Nutritional Sciences, research flied Molecular Nutritional Science.

Jörn M. Schattenberg, M.D., is an Assistant Professor of Medicine at the University Medical Center Mainz, Germany. He received his medical degree after studying at the University of Mainz (Germany) and at the Tulane University in New Orleans (US). He completed his postdoctoral training at the Albert-Einstein College of Medicine, New York, where he focused on delineating signaling pathways involved in the pathophysiology of acute and chronic liver injury with a special focus on metabolic liver disease and NASH. Afterwards he trained in gastroenterology and hepatology and currently serves for in- and out-patients at the University Medical Center in Mainz. He is board-certified in internal medicine and gastroenterology \& hepatology and infectious disease. His current research efforts focus on the optimization of lifestyle interventions for the treatment of NASH, the development of non-invasive diagnostics to stage liver disease and phase II/III clinical trials. 



\section{Preface to "Nutritional Intake and the Risk for Non-Alcoholic Fatty Liver Disease (NAFLD)"}

Non-alcoholic fatty liver disease (NAFLD) is by now one of the most prevalent liver diseases world-wide. General over-nutrition and alterations of the dietary pattern like the consumption of a fat-, cholesterol-, and/or sugar-rich diet are discussed to be critical in the development of NAFLD. While a lot of research efforts have been undertaken throughout the last decades to decipher the molecular mechanisms underlying the development and progression of NAFLD, many aspects are still not fully understood and universally accepted therapies or life-style interventions are not available. Indeed, it has also been suggested that a general reduction of caloric intake especially when combined with an increase of physical activity may lead to an improvement of liver health in NAFLD patients over time when being followed. Yet, results of some epidemiological and experimental studies suggest that dietary composition e.g., partitioning and source of macronutrients may also largely impact disease development and progression and that targeting dietary composition through modulating fat and/ or sugar intake may have beneficial effects on liver status in settings of NAFLD. Also, in focusing on the combination of diet and exercise, not all patients that achieved weight loss resolved their liver disease. Furthermore, in more recent years the intake of pre- and probiotics but also specific micronutrients or secondary plant compounds are also discussed in the prevention and therapy of this disease. However, our understanding of the interaction of nutritional intake, dietary pattern and the development but also prevention and cure of NAFLD is still very limited.

Providing a better understanding of the effects of diet and herein especially of specific macroand micronutrients as well as pre- and probiotics and secondary plant compounds in the context of the development of NAFLD and its progression will help to develop novel prevention and therapeutic strategies for this metabolic liver disease. Therefore, this Special Issue book through including original research and scientific perspectives on the relationship between NAFLD and dietary constituents that aims to provide an in-depth insight in 1) mechanisms involved in the development of the disease and 2) novel prevention strategies to target disease onset and progression. Mechanistic insights defining the contribution of certain nutritional factors to the occurrence and management of NAFLD will improve our understanding of the disease and eventually lead to the development of universally accepted prevention and therapeutic strategies.

Ina Bergheim, Jörn M. Schattenberg Special Issue Editors 



\title{
ChREBP Rather Than SHP Regulates Hepatic VLDL Secretion
}

\author{
Hiroyuki Niwa ${ }^{1}\left(\mathbb{D}\right.$, Katsumi Iizuka ${ }^{1,2, *} \mathbb{1}$, Takehiro Kato ${ }^{1}$, Wudelehu $\mathrm{Wu}^{1}{ }^{1}$, Hiromi Tsuchida ${ }^{1}$, \\ Ken Takao ${ }^{1}$, Yukio Horikawa ${ }^{1}$ and Jun Takeda ${ }^{1}$ \\ 1 Department of Diabetes and Endocrinology, Graduate School of Medicine, Gifu University, Gifu 501-1194, \\ Japan; hiroyu760202@yahoo.co.jp (H.N.); bado_aberu@yahoo.co.jp (T.K.); wudelehu100@126.com (W.W.); \\ Gif095@gifu-u.ac.jp (H.T.); lamgerrpard@yahoo.co.jp (K.T.); yhorikaw@gifu-u.ac.jp (Y.H.); \\ jtakeda@gifu-u.ac.jp (J.T.) \\ 2 Gifu University Hospital Center for Nutritional Support and Infection Control, Gifu 501-1194, Japan \\ * Correspondence: kiizuka@gifu-u.ac.jp; Tel.: +81-58-230-6564; Fax: +81-58-230-6376
}

Received: 6 February 2018; Accepted: 5 March 2018; Published: 7 March 2018

\begin{abstract}
The regulation of hepatic very-low-density lipoprotein (VLDL) secretion plays an important role in the pathogenesis of dyslipidemia and fatty liver diseases. VLDL is controlled by hepatic microsomal triglyceride transfer protein (MTTP). Mttp is regulated by carbohydrate response element binding protein (ChREBP) and small heterodimer partner (SHP). However, it is unclear whether both coordinately regulate Mttp expression and VLDL secretion. Here, adenoviral overexpression of ChREBP and SHP in rat primary hepatocytes induced and suppressed Mttp mRNA, respectively. However, Mttp induction by ChREBP was much more potent than suppression by SHP. Promoter assays of Mttp and the liver type pyruvate kinase gene revealed that SHP and ChREBP did not affect the transcriptional activity of each other. Mttp mRNA and protein levels of Shp ${ }^{-1-}$ mice were similar to those of wild-types; however, those of $\mathrm{Chrebp}^{-/-} \mathrm{Shp}^{-/-}$and $\mathrm{Chrebp}^{-/-}$mice were significantly much lower. Consistent with this, the VLDL particle number and VLDL secretion rates in $\mathrm{Shp}^{-/-}$mice were similar to wild-types but were much lower in Chrebp ${ }^{-/-}$and Chrebp ${ }^{-/-} \mathrm{Shp}^{-/-}$ mice. These findings suggest that ChREBP, rather than SHP, regulates VLDL secretion under normal conditions and that ChREBP and SHP do not affect the transcriptional activities of each other.
\end{abstract}

Keywords: carbohydrate response element binding protein; small heterodimer partner; microsomal triglyceride transfer protein; very-low-density lipoprotein

\section{Introduction}

Nonalcoholic fatty liver disease (NAFLD) is the most common chronic liver disease in the world [1,2]. High fat diets and high carbohydrate diets are responsible in the development of NAFLD [3-5]. High carbohydrate diets cause fatty liver by increasing hepatic de novo lipogenesis [3]. High fat diets increase hepatic fat content [4]. Therefore, lifestyle modifications, including hypocaloric diets, are critical for the prevention and treatment of NAFLD. Non-alcoholic fatty liver disease (NAFLD) is associated with hepatic insulin resistance and hepatic fibrosis and can lead to the development of diabetes mellitus, dyslipidemia, non-alcoholic steatohepatitis, and hepatocellular carcinoma [1,2]. Hepatic lipid accumulation is regulated by the free fatty acid (FFA) supply from adipose tissue, and de novo lipogenesis from the secretion of glucose, acyl CoA oxidation, and very-low-density lipoprotein (VLDL) in the liver [6]. Visceral adipose tissue is the primary source of hepatic fat in adults, contributing $59 \%$ of the triglycerides found in the liver [6]. Glucose and insulin signals regulate hepatic de novo lipogenesis through the carbohydrate response element binding protein (ChREBP) and sterol response element binding protein 1c (SREBP1c), respectively [7]. Moreover, altered VLDL secretion also contributes to the pathogenesis of NAFLD [8,9]. VLDL secretion is 
controlled by microsomal triglyceride transfer protein (MTTP) [8,9]. MTTP is essential for the assembly and secretion of apolipoprotein B-containing lipoproteins. In humans and mice, Mttp deficiency causes hypolipidemia and fatty liver [8,9]. Thus, Mttp is involved in the pathogenesis of NAFLD.

Mttp regulation depends on a few highly-conserved cis-elements in its promoter. The Mttp promoter sequence contains critical positive (hepatic nuclear factor [HNF]-1, HNF-4, direct repeat 1 , and FOX) and negative regulatory sterol and insulin response elements $[8,9]$. Small heterodimer partner (SHP, also known as NR0B2) is a unique nuclear receptor (NR) that contains the dimerization and ligand-binding domain found in other family members but lacks the conserved DNA-binding domain [10]. We previously reported that human SHP genetic variations appear to cause mild obesity and type 2 diabetes mellitus [11,12]. As a co-repressor, SHP represses the activities of HNF- $4 \alpha$ and the retinoid $X$ receptor liver receptor homolog-1 (LRH-1) by interacting with these factors $[13,14]$. However, it is not certain whether SHP affects the VLDL secretion rate $[15,16]$.

As with the effects of nutritional conditions on Mttp expression, high fat diets and high carbohydrate diets induce Mttp expression, resulting in hyperlipidemia [17,18]. High fat diets increase hepatic Mttp expression through decreased binding of sterol regulatory element-binding proteins to the Mttp promoter [17]. High sucrose and high fructose consumption also increase Mttp expression [18]; however, the mechanisms of this are unclear. Recently, we reported that Mttp mRNA levels and VLDL secretion rates were lower in the livers of Carbohydrate Response Element Binding Protein [ChREBP] knockout $\left(\mathrm{Chrebp}^{-/-}\right)$mice [19]. ChREBP is a glucose-activated transcription factor that regulates hepatic de novo lipogenesis in the liver [20-23]. ChREBP transcriptional activities are regulated by phosphorylation/dephosphorylation, nuclear translocation, and conformational changes [20-23]. It was recently proposed that ChREBP transcriptional activity is regulated through interactions with nuclear factors, such as farnesoid X receptor (FXR) and HNF4a [24-28]. Moreover, computer analysis revealed that ChREBP contains a nuclear receptor binding motif [29]. Considering that SHP represses the activities of many transcription factors by interacting with these factors [10], we speculated that SHP might also affect $M t t p$ transcription in cooperation with ChREBP.

ChREBP and SHP control the regulation of Mttp expression. Using rat hepatocytes and knockout mice, we therefore evaluated the following: (1) whether ChREBP and SHP affect the transcriptional activities of each other; (2) whether ChREBP and SHP coordinately affect Mttp expression and thereby VLDL secretion; and (3) if ChREBP or SHP regulate VLDL secretion more potently. An appreciation of the roles of ChREBP and SHP in regulating Mttp and VLDL secretion will be beneficial for understanding the role of nutritional signals in the development of NAFLD and dyslipidemia.

\section{Materials and Methods}

\subsection{Establishment of Chrebp ${ }^{-/-}$Shp ${ }^{-/-}$Double Knockout (DKO) Mice}

Animal experiments were carried out in accordance with the National Institute of Health Guide for the Care and Use of Laboratory Animals (NIH Publications No. 8023, revised 1978). All animal care was approved by the animal care committee of the University of Gifu (No. 27-30, approval date: 4 June 2015). Mice were housed at $23^{\circ} \mathrm{C}$ on a 12 -h light/dark cycle. Chrebp ${ }^{-/-}$mice were backcrossed for at least 10 generations onto the C57BL/6J background $[19,30]$. Shp ${ }^{-/-}$mice were purchased from Lexicon Genetics Inc. (The Woodlands, TX, USA). Shp ${ }^{+/-}$mice were backcrossed for at least 12 generations onto the C57BL/6J background. Male mice were used for all studies. Chrebp ${ }^{-1-} \mathrm{Shp}^{-/-}$ (DKO) mice were intercrossed with $\mathrm{Chrebp}^{-/-}$and $\mathrm{Shp}^{-/-}$mice.

Mice had free access to water and were fed an autoclaved CE-2 diet (CLEA Japan, Tokyo, Japan). Wild-type (WT), Chrebp ${ }^{-/-}, \mathrm{Shp}^{-/-}$, and DKO mice were housed separately with a total of three mice per cage. Body weight was measured weekly between 7 and 21 weeks of age. Mice were sacrificed at 21 weeks of age by cervical dislocation. All tissue samples were immediately placed into liquid nitrogen and stored at $-80^{\circ} \mathrm{C}$ until further analysis for hepatic triacylglycerol and cholesterol contents and for quantitative polymerase chain reaction (PCR). 


\subsection{Liver Triglyceride and Cholesterol Content and Plasma Profile Measurements}

Liver lipids were extracted using the Bligh and Dyer method [31]; they were measured using triglyceride (Wako Pure Chemicals, Osaka, Japan) and cholesterol E-tests (Wako). Blood plasma was collected from the retro-orbital venous plexus, following ad libitum feeding or after a 6-h fast. Blood glucose and beta-hydroxybutyrate $(\beta-\mathrm{OHB})$ levels were measured using a FreeStyle Freedom monitoring system (Nipro, Osaka, Japan). Plasma insulin, FFA, fibroblast growth factor 21 (FGF21), triglyceride, and total cholesterol levels were determined using commercial assay kits, as follows: mouse insulin enzyme-linked immunosorbent assay (ELISA) (H type) (Shibayagi, Gunma, Japan), NEFA C-test (Wako Pure Chemicals, Tokyo, Japan), mouse/rat Fgf21 ELISA (R\&D Systems, Minneapolis, MN, USA), triglyceride E-test (Wako), and the cholesterol E-test (Wako), respectively.

\subsection{RNA Isolation and Quantitative Real-Time PCR}

Total RNA isolation, cDNA synthesis, and real-time PCR analysis were performed as previously described [13]. Real-time PCR primers for mouse/rat Chrebp, liver type pyruvate kinase (Pklr), Fgf21, Mttp, and RNA polymerase II (Pol2) have been previously reported [31-33]. All amplifications were performed in triplicate. The relative amounts of mRNA were calculated using the comparative CT method. Pol2 expression was used as an internal control.

\subsection{VLDL Secretion Test and MTTP Protein Contents}

VLDL secretion tests were performed as previously reported [19]. Briefly, $500 \mathrm{mg} / \mathrm{kg}$ body weight tyloxapol was administered intraperitoneally to $5 \mathrm{~h}$ fasted mice. Blood sampling was performed at the indicated times. The triglyceride (TG) content in lipoprotein fractions and the VLDL particle numbers were analyzed using gel-permeation high-performance liquid chromatography (LipoSEARCH ${ }^{\circledR}$ ) at Skylight Biotech Inc. (Akita, Japan) [34]. Serum samples obtained from six mice after $6 \mathrm{~h}$ fasting were pooled and measured. Liver MTTP protein contents were measured by an MTTP ELISA kit (Cloud-Clone Corp Inc., Houston, TX, USA).

\subsection{Adenoviral Delivery into Rat Primary Hepatocytes}

Rat primary hepatocytes were isolated as previously described [32,33]. Isolated hepatocytes were suspended in DMEM, supplemented with 10\% fetal calf serum, $100 \mathrm{nM}$ insulin, $100 \mathrm{nM}$ dexamethasone, $10 \mathrm{nM}$ triiodothronine (T3), and $100 \mu \mathrm{g} / \mathrm{mL}$ penicillin/streptomycin. Cells were seeded in 6-well plates or $10-\mathrm{cm}$ dishes and grown in a humidified atmosphere of $5 \% \mathrm{CO}_{2}$ and $95 \%$ air at $37^{\circ} \mathrm{C}$. After the cells had been incubated for $4 \mathrm{~h}$, the medium was replaced with DMEM containing $10 \mathrm{nM}$ T3. Adenoviruses harboring dominant active rat Chrebp lacking 1-196 a.a. (Ad-daChREBP) and mouse SHP full length (Ad-SHP) were constructed according to the manufacturer's protocol (Invitrogen, Carlsbad, CA, USA). After $4 \mathrm{~h}$ infection with Ad-daChREBP and/or Ad-SHP, rat hepatocytes were incubated for $20 \mathrm{~h}$. Mttp mRNA levels were detected by real-time PCR.

\subsection{Transfections and Luciferase Reporter Assay}

Reporter plasmids pGL3 $3 \times$ LPK ChoRE, pcDNA-daChrebp, and pcDNA-empty have been previously reported [32,33]. pcDNA-SHP and pcDNA-FXR were constructed using the pcDNA ${ }^{\mathrm{TM}} 3.2-\mathrm{DEST}$ Mammalian Expression Vector (Invitrogen) according to the manufacturer's protocol (Invitrogen). pGL3-Mttp (-211 bp) was constructed as follows: mouse Mttp promoter regions ( $-211 \mathrm{bp}$ to $+77 \mathrm{bp}$ ) amplified by PrimSTAR MAX DNA Polymerase (Takara Bio, Kusatsu, Japan) were inserted into the pGL3 basic vector (Promega, Madison, WI, USA). Then, $1.0 \mu \mathrm{g}$ of pGL3 MTTP (-211 bp), $0.1 \mu \mathrm{g}$ pRL-TK vector, pcDNA-empty + pcDNA-daChREBP + pcDNA-SHP (total $1.0 \mu \mathrm{g}$ ), and $3 \mu \mathrm{L}$ of Lipofectamine 2000 (Invitrogen) were transfected into primary rat hepatocytes. After incubation for $24 \mathrm{~h}$, cells were collected and used in luciferase assays. 


\subsection{Statistical Analysis}

All values are presented as means \pm standard deviations. Data were analyzed using Tukey's test. A $p$-value $<0.05$ was considered to be statistically significant.

\section{Results}

\subsection{Adenoviral Overexpression of ChREBP and SHP Respectively Increased and Decreased Mttp Expression}

Adenoviral ChREBP caused an increase in Mttp and Pklr mRNA expression, while SHP suppressed only Mttp expression in primary rat hepatocytes (Figure 1A,B). Moreover, overexpressing SHP by more than 70-fold (above physiological levels) was found to suppress ChREBP-mediated Mttp induction (Figure 1A). In contrast, SHP overexpression failed to suppress ChREBP-mediated Pklr induction (Figure 1B). This suggested that ChREBP induced Mttp expression more potently than SHP suppressed it.

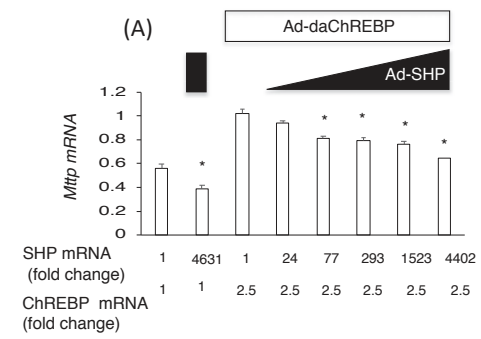

(C)

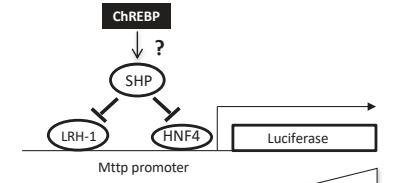

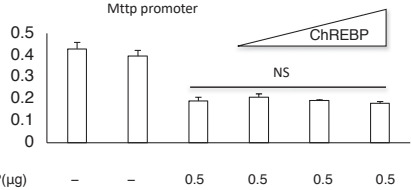

$\begin{array}{lllllll}\operatorname{pcDNASHP}(\mu g) & - & - & 0.5 & 0.5 & 0.5 & 0.5 \\ \operatorname{pcDNA}- & & & & & & \end{array}$

(B)

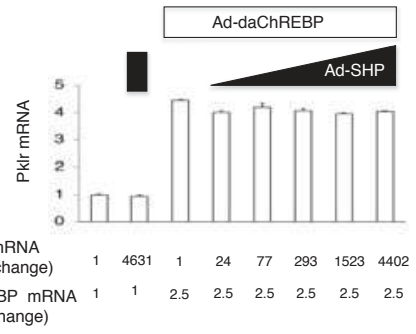

(D)

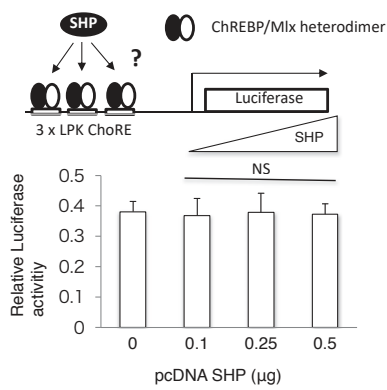

Figure 1. The coordinated effects of carbohydrate response element binding protein (ChREBP) and small heterodimer partner (SHP) on Mttp expression. (A) and (B) The effects of SHP on ChREBP-mediated Mttp (A) and Pklr (B) mRNA induction. After $4 \mathrm{~h}$ infection with Ad-daChREBP and/or Ad-SHP in hepatocytes, cells were incubated for $20 \mathrm{~h}$. Mttp mRNA levels were detected by real-time Polymerase chain reaction method. Open symbols and closed symbols indicated adenoviral overexpression of daChREBP and SHP, respectively. The $x$-axis indicates Shp and Chrebp mRNA levels (fold change). Adenoviral overexpression of ChREBP caused a 2.5-fold increase in Chrebp mRNA levels. Adenoviral SHP expression caused an indicated fold increase in Shp expresssion. Pol2 expression was used as an internal control. $n=4$ per group. ${ }^{*} p<0.05$; (C) Reporter assay using pGL3-Mttp ( $\left.-211 \mathrm{bp}\right)$. The indicated amounts of pcDNA-daChREBP and pcDNA-SHP were transfected with pGL3-Mttp $(-211 \mathrm{bp})$ and pRL-TK vectors and Lipofectamine 2000 reagent into primary rat hepatocytes. After $24 \mathrm{~h}$ incubation, cells were collected for the dual luciferase assay. Luciferase activity was normalized to Renilla luciferase activity. N.S., not significant. $n=6$ per group; (D) Reporter assay using pGL3 $3 \times$ LPK ChoRE. The indicated amount of pcDNA-SHP was cotransfected with pGL3-3 $\times$ LPK ChoRE, PRL-TK, pcDNA daChREBP, and Lipofectamine 2000 into primary rat hepatocytes. After $24 \mathrm{~h}$ incubation, cells were collected for the dual luciferase assay. Luciferase activity was normalized to Renilla luciferase activity. NS, not significant vs control. $n=6$ per group. 
SHP is known to suppress HNF4/HNF1/LRH-1-mediated Mttp expression [13,35]. Accordingly, transfection of the PGL3 basic vector containing 211 bp of the Mttp promoter region (pcDNA-SHP), including the HNF4/HNF1/LRH-1 binding site, successfully suppressed PGL3-Mttp (-211 bp) luciferase activity. However, co-transfection of pcDNA-daChREBP did not reverse this effect (Figure 1C). In contrast, the transfection of the pcDNA SHP vector could not suppress pGL3 Pklr luciferase activities induced by pcDNA-daChREBP (Figure 1D). This suggested that SHP and ChREBP did not affect the transcriptional activities of each other

\subsection{Chrebp Shp DKO Mice Resembled Chrebp ${ }^{-/-}$Mice}

We next assessed the effects of ChREBP and SHP on the mouse metabolic phenotype in vivo. Chrebp $^{-/-}$mice displayed characteristically higher liver weights, lower white adipose tissue weights, higher plasma FFA, FGF-21, and $\beta$-OHB levels, and elevated liver glycogen contents compared with those of WT mice. Chrebp ${ }^{-/-} \mathrm{Shp}^{-/-}$double knockout (DKO) mice also displayed similar characteristics (Table 1).

Table 1. Phenotypic comparison between WT, $\mathrm{ChREBP}^{-/-}, \mathrm{SHP}^{-/-}, \mathrm{ChREBP}^{-/-} \mathrm{SHP}^{-/-}$(DKO) mice.

\begin{tabular}{|c|c|c|c|c|}
\hline & WT & ChREBP $^{-1-}$ & $\mathrm{SHP}^{-1-}$ & DKO \\
\hline Liver $(\% \mathrm{BW})$ & $5.02 \pm 0.08$ & $5.37 \pm 0.27 *$ & $5.01 \pm 0.14$ & $5.52 \pm 0.24 *$ \\
\hline Mesenteric fat (\%BW) & $1.16 \pm 0.13$ & $0.77 \pm 0.16 *$ & $1.02 \pm 0.28$ & $0.55 \pm 0.11 *$ \\
\hline Brown adipose tissue (\%BW) & $0.30 \pm 0.07$ & $0.27 \pm 0.10$ & $0.36 \pm 0.12$ & $0.49 \pm 0.15$ \\
\hline Plasma glucose $(\mathrm{mg} / \mathrm{dL})$ & $132.3 \pm 17.3$ & $128.4 \pm 13.2$ & $118.7 \pm 21.5$ & $117.2 \pm 11.5$ \\
\hline HOMA-R & $0.28 \pm 0.08$ & $0.32 \pm 0.12$ & $0.27 \pm 0.15$ & $0.19 \pm 0.03$ \\
\hline Plasma triglyceride (mg/dL) & $66.1 \pm 10.1$ & $56.2 \pm 11.5$ & $65.2 \pm 12.6$ & $67.3 \pm 19.8$ \\
\hline Plasma FGF21 (pg/mL) & $802.1 \pm 365.8$ & $150.2 \pm 51.1 *$ & $584.1 \pm 268.6$ & $124.0 \pm 61.8 *$ \\
\hline Plasma $\beta$-hydroxybutyrate (mM) & $2.05 \pm 0.24$ & $1.32 \pm 0.11$ & $2.37 \pm 0.40$ & $1.49 \pm 0.22$ \\
\hline Liver glycogen content (mg/g liver) & $40.5 \pm 12.4$ & $80.8 \pm 20.2 *$ & $33.4 \pm 10.5$ & $78.0 \pm 22.7 *$ \\
\hline
\end{tabular}

BW, body weight; EP, epidydimal fat weight; VS, visceral fat weight; BAT, brown adipose tissue weights; FGF21, fibroblast growth factor $21 .{ }^{*} p<0.05$ vs. WT.

Chrebp and Shp mRNA levels were unaffected by Shp and Chrebp deletions, respectively (Figure 2A,B). Consistent with these phenotypes, the expression levels of ChREBP target genes, such as Pklr and Fgf21, were significantly lower in $\mathrm{Chrebp}^{-/-}$and DKO mice (Figure 2C,D). In contrast, the expression of Cyp7a1, a SHP target gene, was significantly higher in Shp ${ }^{-/-}$and DKO mice but was unchanged in Chrebp ${ }^{-/-}$mice (Figure 2E). Many phenotypes seen in DKO mice were similar to those of Chrebp ${ }^{-/-}$mice, suggesting that SHP had little effect on ChREBP target gene expression. 
(A)

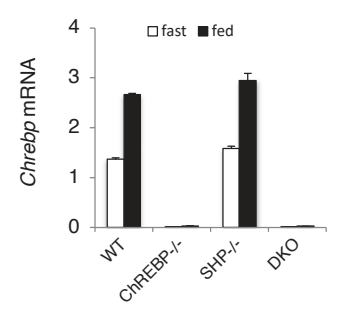

(D)

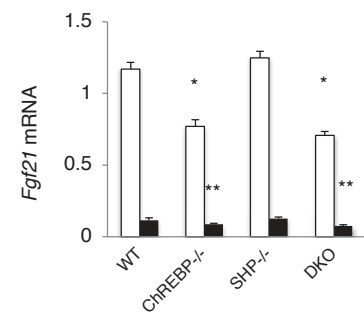

(B)

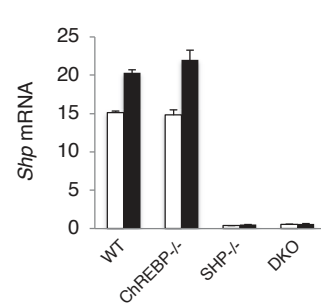

(C)

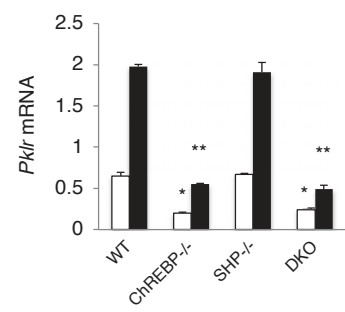

(E)

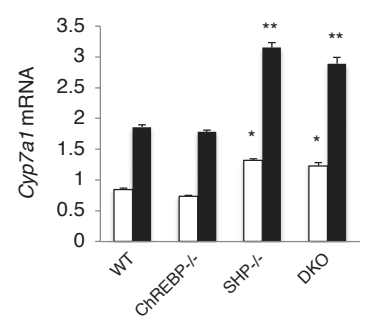

Figure 2. ChREBP and SHP target mRNA levels in wild-type, Chrebp ${ }^{-/-}$, Shp ${ }^{-/-}$, and Chrebp ${ }^{-1-}$ $\mathrm{Shp}^{-1-}$ mice. Carbohydrate response element binding protein (Chrebp) (A); small heterodimer partner (Shp) (B); liver type pyruvate kinase (Pklr) (C); fibroblast growth factor 21 (Fgf21) (D); and Cholesterol 7 alpha-hydroxylase (Cyp7a1); (E) mRNA expression analysis in the livers of wild-type, Chrebp ${ }^{-1-}$, $\mathrm{Shp}^{-1-}$, and $\mathrm{Chrebp}^{-1-} \mathrm{Shp}^{-1-}$ (DKO) mice. Open squares indicate fasted conditions; closed squares indicate fed conditions. Pol2 expression was used as an internal control. Each mRNA levels were corrected with mouse RNA polymerase 2 mRNA. ${ }^{*} p<0.05$ vs. WT (fast), $n=4 .{ }^{* *} p<0.05$ vs. WT (fed), $n=4$.

\subsection{ChREBP and SHP Respectively Positively and Negatively Controlled VLDL Secretion through Mttp Regulation}

We evaluated the effects of Chrebp and Shp deletion on Mttp expression. Hepatic Mttp mRNA levels were much lower in Chrebp ${ }^{-/-}$and DKO mice than in WT mice; however, those in Shp ${ }^{-/-}$ mice were only slightly (only 1.15 times) higher than in WT mice (Figure 3A). Consistent with mRNA levels, MTTP protein levels were significantly much lower in $\mathrm{Chrebp}^{-/-}$and DKO mice than in WT mice (Figure 3B). Moreover, VLDL TG contents of $\mathrm{Chrebp}^{-/-}$and DKO mice were lower than those in WT and Shp ${ }^{-/-}$mice, while VLDL secretion rates in Chrebp ${ }^{-1-}$ and DKO mice were approximately 0.6 times lower than in WT mice (Figure 3C). In contrast, liver VLDL secretion rates in Shp ${ }^{-/}$mice were similar to those in WT mice (Figure 3C). In support of this, the VLDL TG contents and VLDL particle numbers in $\mathrm{Chrebp}^{-1-}$ mice and DKO mice were much lower those in WT mice, while those in Shp $\mathrm{p}^{-/-}$mice were similar to those in WT mice (Figure 3D,E). Therefore, under normal conditions, the effect of ChREBP on Mttp expression was potent but the effect of SHP was physiologically much weaker or lacking. 
(A)

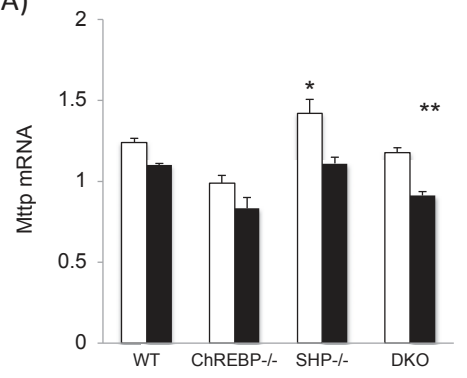

(B)

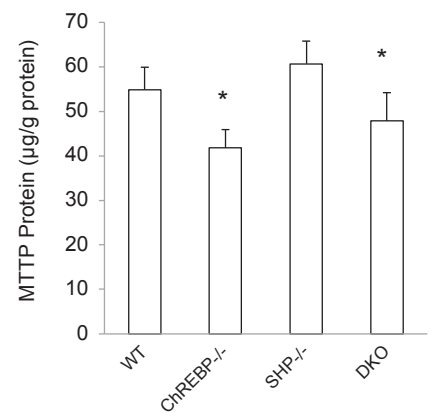

(E)
(C)

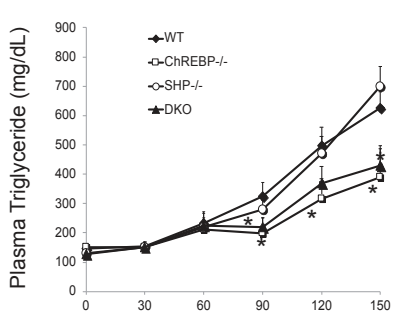

(D)

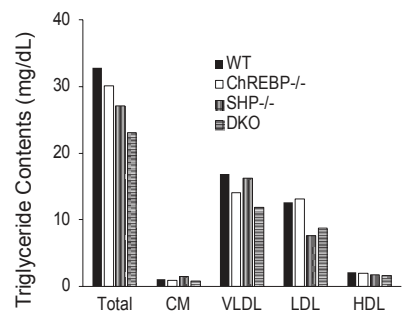

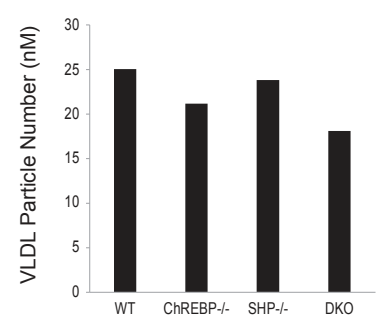

Figure 3. Shp deletion failed to recover decreased Mttp expression, and thereby, VLDL secretion rates mediated by Chrebp deletion. (A) Hepatic Mttp mRNA levels in the livers of wild-type (WT), $\mathrm{Chrebp}^{-/-}, \mathrm{Shp}^{-/-}$, and DKO mice. Mttp mRNA levels were detected by real-time PCR. Pol2 expression was used as an internal control. ${ }^{*} p<0.05$ vs. WT (fast), $n=4 .{ }^{* *} p<0.05$ vs. WT (fed), $n=4$. Data are represented as means $\pm \mathrm{SD}$; (B) Hepatic MTTP protein expression in the livers of WT, $\mathrm{Chrebp}^{-1-}, \mathrm{Shp}^{-1-}$, and DKO mice. MTTP protein levels were measured by ELISA and corrected for total protein levels. ${ }^{*} p<0.05$ vs. WT, $n=5-7$. Data are represented as means \pm SD; (C) VLDL secretion rates were measured as previously reported [17]. ${ }^{*} p<0.05$ vs. WT, $n=5-7$. Data are represented as means \pm SD; TG contents in lipoprotein fraction (D) and VLDL particle numbers (E) were analyzed using gel-permeation high-performance liquid chromatography. Data are representative of six samples pooled.

\section{Discussion}

In this study, we evaluated whether ChREBP and SHP could coordinately affect VLDL secretion via Mttp expression. ChREBP and SHP reciprocally affected Mttp mRNA levels; however, the potency of Mttp suppression by SHP was much lower than that of Mttp induction by ChREBP. Therefore, ChREBP and SHP did not affect the transcriptional activity of each other. Mttp mRNA and protein levels in $\mathrm{Chrebp}^{-/-}$and DKO mice were much lower than those in WT mice; however, those in Shp ${ }^{-/-}$ mice were similar to WT. In agreement with this, VLDL secretion rates of DKO and Chrebp ${ }^{-1-}$ mice were much lower than those of Shp ${ }^{-/-}$mice, which were the same as those of WT mice. Together, these findings suggest that ChREBP, rather than SHP, regulates Mttp expression under normal conditions.

SHP predominantly functions as a transcriptional repressor of gene expression that binds directly to nuclear receptors, such as LRH-1, HNF $4 \alpha$, estrogen receptors, estrogen receptor-related receptors, liver $\mathrm{X}$ receptors, peroxisome proliferator-activated receptors, glucocorticoid receptor, thyroid hormone receptor $\beta$, retinoic acid receptor $\alpha, F X R$, pregnane $X$ receptor, constitutive androstane receptor, androgen receptor, nerve growth factor IB, and common heterodimerization partner retinoid $X$ receptors [10]. SHP was previously shown to suppress MTTP expression by binding to HNF4 $\alpha /$ LRH-1 sites in the Mttp promoter [10,13,35]. 
Recent reports have proposed a new ChREBP regulatory mechanism (ChREBP-nuclear receptor interaction), in which nuclear factors interact with ChREBP to modify ChREBP transcriptional activity [25-28]. Computer analysis previously revealed that ChREBP contains an LxQLLT sequence that matches the NR binding motif [29]. The rat LxQLLT sequence is localized to a proline-rich region (540-545 a.a.) of ChREBP [29]. SHP binds and represses the transcriptional activities of target genes by utilizing two functional LXXLL-related motifs located in the ligand-binding domain [10]. Some studies have also suggested that the interactions between ChREBP and nuclear receptors, such as FXR and HNF4, play physiological roles in regulating Pklr expression [25-28]. HNF4 and FXR positively and negatively regulate ChREBP transcriptional activity, respectively. However, our data revealed that SHP overexpression did not affect Pklr mRNA or luciferase activity of the PGL3 3xPKLR ChoRE vector. Moreover, SHP deletion did not affect the mRNA levels of ChREBP target genes ( $P k l r$ and Fgf21) in mice. Consistent with this, we found that ChREBP interacted with SHP in the mammalian two-hybrid system, and interactions with SHP did not interfere with the binding between FXR and ChREBP in the mammalian two-hybrid system (data not shown). This indicated that SHP might not affect the interplay between ChREBP and nuclear factors such as FXR and HNF4, and therefore, that it does not modulate ChREBP transcriptional activity.

Reporter assays for the Mttp promoter $(-211 /+81 \mathrm{bp})$ in the present study revealed that SHP suppressed hepatic Mttp expression, but that ChREBP overexpression did not affect luciferase activity controlled by the Mttp promoter ( $-211 /+81 \mathrm{bp}$ ). This supports the notion that ChREBP does not affect the role of SHP as a corepressor. Taken together with the fact that a Chrebp deletion did not affect SHP target gene (Cyp7a1) mRNA levels, this suggested that ChREBP does not modulate SHP transcriptional activity. Thus, ChREBP and SHP do not affect the transcriptional activities of each other.

To evaluate ChREBP binding to Mttp promoter regions, we searched for putative ChoRE motifs [36-38] in the promoter, exons, and introns. However, we did not detect ChoREs in Mttp. Considering that ChREBP regulates the expression of many genes [21,30,32,33,36-39], we propose that it might indirectly induce Mttp expression through the activation of other transcription factors. However, further investigation is needed to identify the mechanism underlying the ChREBP induction of Mttp expression.

The VLDL secretory pathway is regulated by MTTP, a rate-limiting enzyme. We previously reported that ChREBP positively regulates VLDL secretion [19]. Although SHP controls Mttp expression by modulating HNF4 and LRH-1 transcriptional activity, its effect on VLDL secretion was controversial $[15,16]$. Some studies reported that VLDL secretion in $\mathrm{Shp}^{-/-}$mice on ob/ob or C57BL/ 6 backgrounds increased relative to WT mice [16], while others showed that VLDL secretion in $\mathrm{Sh}^{-/-}$mice fed a Western diet was similar to that in Shp ${ }^{-/-}$mice fed a normal diet [15]. Our data were consistent with the latter. They also confirmed that ChREBP regulates VLDL secretion, but that SHP deletion did not affect VLDL secretion in either WT or Chrebp ${ }^{-/-}$mice. Our analyses of MTTP protein levels and VLDL particle numbers also supported these data. Taken together with the fact that SHP overexpression by more than 70 times was needed to suppress ChREBP-mediated Mttp induction, these findings suggest that ChREBP plays important roles in VLDL secretion, while those of SHP are much smaller or negligible.

Nutritional conditions affect the development of NAFLD and dyslipidemia. High carbohydrate diets and high fat diets increase Mttp expression [9]. High carbohydrate diets increase de novo lipogenesis and Mttp expression [9,18]. As we previously reported, Chrebp deletions prevent hepatic lipid accumulation following consumption of a high carbohydrate diet $[30,40]$. The prevention from high carbohydrate diet induced fatty liver was due to a decrease in de novo lipogenesis, decreased free fatty acid supply from adipose tissue, and appetite loss from the high sucrose and high fructose diet [30,40-43]. Decreased Mttp expression might deteriorate NAFLD in ChREBP ${ }^{-/-}$mice. As ChREBP ${ }^{-/-}$mice could not be fed a high carbohydrate diet such as the high sucrose diet [30,41,42], we could not perform an experiment using the high carbohydrate diet. In contrast, a high fat diet increased the FFA supply from dietary fat and induced Mttp expression [17]. The High fat diet 
suppressed ChREBP transcriptional activity [44]. Moreover, Chrebp deletion did not prevent from high fat diet induced fatty liver owing to decreased fatty acid oxidation [19,43]. As Shp suppress PPAR alpha [45], Shp deletion prevented hepatic lipid accumulation through increased fatty acid oxidation [15]. Consistent with our data, Mttp expression was not affected by Shp deletion. Therefore, we analyzed the effects of ChREBP and SHP deletion on Mttp expression and VLDL secretion under a normal diet condition. In this study, ChREBP and SHP independently regulated glucose and lipid metabolism. The difference in target genes between ChREBP and SHP might contribute to the different effects of ChREBP and SHP on preventing the development of fatty liver induced by high fat diets in Chrebp $^{-1-}$ and Shp ${ }^{-/-}$mice (Figure 4).

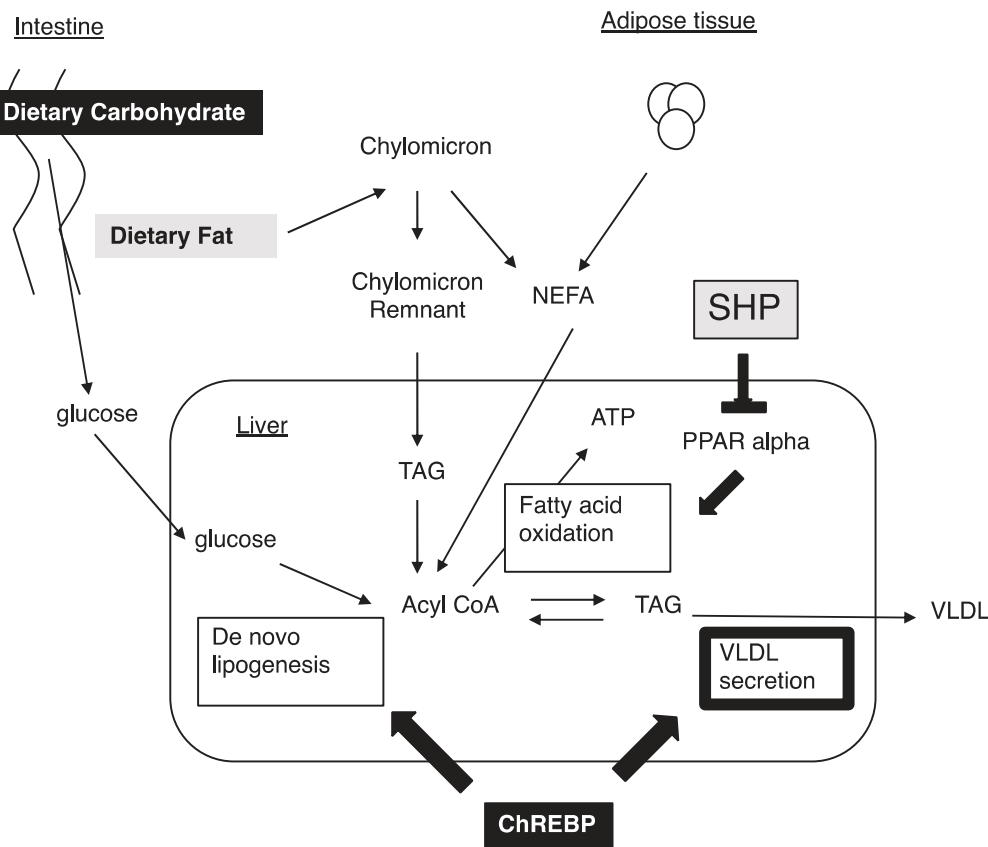

Figure 4. ChREBP and SHP independently regulate hepatic lipid metabolism. High carbohydrate diets increase de novo lipogenesis and VLDL secretion through ChREBP activation. Therefore, Chrebp deletion prevents high carbohydrate diet induced fatty liver [30,40,42,43]. In contrast, SHP suppresses PPAR alpha and an Shp deletion prevents high fat diet induced fatty liver [15,45]. In this study, we clarified that ChREBP, rather than SHP, regulates VLDL secretion through Mttp expression (bold line). Thus, ChREBP and SHP independently regulate hepatic lipid metabolism.

\section{Conclusions}

ChREBP and SHP were shown to modulate hepatic Mttp expression, but the capacity of Mttp suppression by SHP was much lower than that of Mttp induction by ChREBP. Moreover, ChREBP and SHP did not affect the transcriptional activities of each other. Unlike many transcription factors, ChREBP was not regulated by SHP. Finally, ChREBP, rather than SHP, was shown to regulate hepatic VLDL secretion.

Acknowledgments: We thank Sarah Williams, from Edanz Group (www.edanzediting.com) for editing a draft of this manuscript. This work was supported in part by a Grant-in-Aid for Scientific Research from the Japan Society for the Promotion of Science (Iizuka, K.: No. 17K00850, 26500005, Takeda, J.: No. 17K19902), research grants from MSD and Novartis Pharma. (Iizuka, K. and Takeda, J.). 
Author Contributions: K.I. conceived and designed the experiments; H.N., T.K., W.W., H.T. and K.T. performed the experiments; H.N. analyzed the data; Y.H. gave a support in the literature review; K.I. and J.T. wrote and revised the paper. All the authors approved the final version of the manuscript.

Conflicts of Interest: The authors declare no conflicts of interest.

\section{References}

1. Fabbrini, E.; Magkos, F. Hepatic Steatosis as a Marker of Metabolic Dysfunction. Nutrients 2015, 7, 4995-5019. [CrossRef] [PubMed]

2. Fabbrini, E.; Sullivan, S.; Klein, S. Obesity and nonalcoholic fatty liver disease: Biochemical, metabolic, and clinical implications. Hepatology 2010, 51, 679-689. [CrossRef] [PubMed]

3. Agius, L. High-carbohydrate diets induce hepatic insulin resistance to protect the liver from substrate overload. Biochem. Pharmacol. 2013, 85, 306-312. [CrossRef] [PubMed]

4. Westerbacka, J.; Lammi, K.; Häkkinen, A.M.; Rissanen, A.; Salminen, I.; Aro, A.; Yki-Järvinen, H. Dietary fat content modifies liver fat in overweight nondiabetic subjects. J. Clin. Endocrinol. Metab. 2005, 90, 2804-2809. [CrossRef] [PubMed]

5. Yu, J.; Marsh, S.; Hu, J.; Feng, W.; Wu, C. The Pathogenesis of Nonalcoholic Fatty Liver Disease: Interplay between Diet, Gut Microbiota, and Genetic Background. Gastroenterol. Res. Pract. 2016, 2016, 2862173. [CrossRef] [PubMed]

6. Donnelly, K.L.; Smith, C.I.; Schwarzenberg, S.J.; Jessurun, J.; Boldt, M.D.; Parks, E.J. Sources of fatty acids stored in liver and secreted via lipoproteins in patients with nonalcoholic fatty liver disease. J. Clin. Investig. 2005, 115, 1343-1351. [CrossRef] [PubMed]

7. Browning, J.D.; Horton, J.D. Molecular mediators of hepatic steatosis and liver injury. J. Clin. Investig. 2004, 114, 147-152. [CrossRef] [PubMed]

8. Cuchel, M.; Rader, D.J. Microsomal transfer protein inhibition in humans. Curr. Opin. Lipidol. 2013, 24, 246-250. [CrossRef] [PubMed]

9. Hussain, M.M.; Nijstad, N.; Franceschini, L. Regulation of microsomal triglyceride transfer protein. Clin. Lipidol. 2011, 6, 293-303. [CrossRef] [PubMed]

10. Zhang, Y.; Hagedorn, C.H.; Wang, L. Role of nuclear receptor SHP in metabolism and cancer. Biochim. Biophys. Acta 2011, 1812, 893-908. [CrossRef] [PubMed]

11. Nishigori, H.; Tomura, H.; Tonooka, N.; Kanamori, M.; Yamada, S.; Sho, K.; Inoue, I.; Kikuchi, N.; Onigata, K.; Kojima, I.; et al. Mutations in the small heterodimer partner gene are associated with mild obesity in Japanese subjects. Proc. Natl. Acad. Sci. USA 2001, 98, 575-580. [CrossRef] [PubMed]

12. Enya, M.; Horikawa, Y.; Kuroda, E.; Yonemaru, K.; Tonooka, N.; Tomura, H.; Oda, N.; Yokoi, N.; Yamagata, K.; Shihara, N.; et al. Mutations in the small heterodimer partner gene increase morbidity risk in Japanese type 2 diabetes patients. Hum. Mutat. 2008, 29, E271-E277. [CrossRef] [PubMed]

13. Lee, Y.K.; Dell, H.; Dowhan, D.H.; Hadzopoulou-Cladaras, M.; Moore, D.D. The orphan nuclear receptor SHP inhibits hepatocyte nuclear factor 4 and retinoid $\mathrm{X}$ receptor transactivation: Two mechanisms for repression. Mol. Cell. Biol. 2000, 20, 187-195. [CrossRef] [PubMed]

14. Goodwin, B.; Jones, S.A.; Price, R.R.; Watson, M.A.; McKee, D.D.; Moore, L.B.; Galardi, C.; Wilson, J.G.; Lewis, M.C.; Roth, M.E.; et al. A regulatory cascade of the nuclear receptors FXR, SHP-1, and LRH-1 represses bile acid biosynthesis. Mol. Cell 2000, 6, 517-526. [CrossRef]

15. Park, Y.J.; Kim, S.C.; Kim, J.; Anakk, S.; Lee, J.M.; Tseng, H.T.; Yechoor, V.; Park, J.; Choi, J.S.; Jang, H.C.; et al. Dissociation of diabetes and obesity in mice lacking orphan nuclear receptor small heterodimer partner. J. Lipid Res. 2011, 52, 2234-2244. [CrossRef] [PubMed]

16. Huang, J.; Iqbal, J.; Saha, P.K.; Liu, J.; Chan, L.; Hussain, M.M.; Moore, D.D.; Wang, L. Molecular characterization of the role of orphan receptor small heterodimerpartner in development of fatty liver. Hepatology 2007, 46, 147-157. [CrossRef] [PubMed]

17. Lin, M.C.; Arbeeny, C.; Bergquist, K.; Kienzle, B.; Gordon, D.A.; Wetterau, J.R. Cloning and regulation of hamster microsomal triglyceride transfer protein. The regulation is independent from that of other hepatic and intestinal proteins which participate in the transport of fatty acids and triglycerides. J. Biol. Chem. 1994, 269, 29138-29145. [PubMed] 
18. Mock, K.; Lateef, S.; Benedito, V.A.; Tou, J.C. High-fructose corn syrup-55 consumption alters hepatic lipid metabolism and promotes triglyceride accumulation. J. Nutr. Biochem. 2017, 39, 32-39. [CrossRef] [PubMed]

19. Wu, W.; Tsuchida, H.; Kato, T.; Niwa, H.; Horikawa, Y.; Takeda, J.; Iizuka, K. Fat and carbohydrate in western diet contribute differently to hepatic lipid accumulation. Biochem. Biophys. Res. Commun. 2015, 461, 681-686. [CrossRef] [PubMed]

20. Uyeda, K.; Repa, J.J. Carbohydrate response element binding protein, ChREBP, a transcription factor coupling hepatic glucose utilization and lipid synthesis. Cell Metab. 2006, 4, 107-110. [CrossRef] [PubMed]

21. Iizuka, K. The transcription factor carbohydrate-response element-binding protein (ChREBP): A possible link between metabolic disease and cancer. Biochim. Biophys. Acta 2017, 1863, 474-485. [CrossRef] [PubMed]

22. Abdul-Wahed, A.; Guilmeau, S.; Postic, C. Sweet Sixteenth for ChREBP: Established Roles and Future Goals. Cell Metab. 2017, 26, 324-341. [CrossRef] [PubMed]

23. Herman, M.A.; Samuel, V.T. The Sweet Path to Metabolic Demise: Fructose and Lipid Synthesis. Trends Endocrinol. Metab. 2016, 27, 719-730. [CrossRef] [PubMed]

24. Poupeau, A.; Postic, C. Cross-regulation of hepatic glucose metabolism via ChREBP and nuclear receptors. Biochim. Biophys. Acta 2011, 1812, 995-1006. [CrossRef] [PubMed]

25. Caron, S.; Huaman Samanez, C.; Dehondt, H.; Ploton, M.; Briand, O.; Lien, F.; Dorchies, E.; Dumont, J.; Postic, C.; Cariou, B.; et al. Farnesoid X receptor inhibits the transcriptional activity of carbohydrate response element binding protein in human hepatocytes. Mol. Cell. Biol. 2013, 33, 2202-2211. [CrossRef] [PubMed]

26. Meng, J.; Feng, M.; Dong, W.; Zhu, Y.; Li, Y.; Zhang, P.; Wu, L.; Li, M.; Lu, Y.; Chen, H.; et al. Identification of HNF- $4 \alpha$ as a key transcription factor to promote ChREBP expression in response to glucose. Sci. Rep. 2016, 6, 23944. [CrossRef] [PubMed]

27. Trabelsi, M.S.; Daoudi, M.; Prawitt, J.; Ducastel, S.; Touche, V.; Sayin, S.I.; Perino, A.; Brighton, C.A.; Sebti, Y.; Kluza, J.; et al. Farnesoid X receptor inhibits glucagon-like peptide-1 production by enteroendocrine L cells. Nat. Commun. 2015, 6, 7629. [CrossRef] [PubMed]

28. Burke, S.J.; Collier, J.J.; Scott, D.K. cAMP opposes the glucose-mediated induction of the L-PK gene by preventing the recruitment of a complex containing ChREBP, HNF4alpha, and CBP. FASEB J. 2009, 23, 2855-2865. [CrossRef] [PubMed]

29. McFerrin, L.G.; Atchley, W.R. A novel N-terminal domain may dictate the glucose response of Mondo proteins. PLoS ONE 2012, 7, e34803. [CrossRef] [PubMed]

30. Iizuka, K.; Bruick, R.K.; Liang, G.; Horton, J.D.; Uyeda, K. Deficiency of carbohydrate response element-binding protein (ChREBP) reduces lipogenesis as well as glycolysis. Proc. Natl. Acad. Sci. USA 2004, 101, 7281-7286. [CrossRef] [PubMed]

31. Bligh, E.G.; Dyer, W.J. A rapid method of total lipid extraction and purification. Can. J. Biochem. Physiol. 1959, 37, 911-917. [CrossRef] [PubMed]

32. Iizuka, K.; Horikawa, Y. Regulation of lipogenesis via BHLHB2/DEC1 and ChREBP feedback looping. Biochem. Biophys. Res. Commun. 2008, 374, 95-100. [CrossRef] [PubMed]

33. Iizuka, K.; Takeda, J.; Horikawa, Y. Glucose induces FGF21 mRNA expression through ChREBP activation in rat hepatocytes. FEBS Lett. 2009, 583, 2882-2886. [CrossRef] [PubMed]

34. Okazaki, M.; Yamashita, S. Recent Advances in Analytical Methods on Lipoprotein Subclasses: Caluculation of Particle Numbers from Lipid Levels by Gel Permeation HPLC Using "Spherical Particle Model". J. Oleo Sci. 2016, 65, 265-282. [CrossRef] [PubMed]

35. Hirokane, H.; Nakahara, M.; Tachibana, S.; Shimizu, M.; Sato, R. Bile acid reduces the secretion of very low density lipoprotein by repressing microsomal triglyceridetransfer protein gene expression mediated by hepatocyte nuclear factor-4. J. Biol. Chem. 2004, 279, 45685-45692. [CrossRef] [PubMed]

36. Jeong, Y.S.; Kim, D.; Lee, Y.S.; Kim, H.J.; Han, J.Y.; Im, S.S.; Chong, H.K.; Kwon, J.K.; Cho, Y.H.; Kim, W.K.; et al. Integrated expression profiling and genome-wide analysis of ChREBP targets reveals the dual role for ChREBP in glucose-regulated gene expression. PLoS ONE 2011, 6, e22544. [CrossRef] [PubMed]

37. Poungvarin, N.; Chang, B.; Imamura, M.; Chen, J.; Moolsuwan, K.; Sae-Lee, C.; Li, W.; Chan, L. Genome-Wide Analysis of ChREBP Binding Sites on Male Mouse Liver and White Adipose Chromatin. Endocrinology 2015, 156, 1982-1994. [CrossRef] [PubMed]

38. Ma, L.; Robinson, L.N.; Towle, H.C. ChREBP*Mlx is the principal mediator of glucose-induced gene expression in the liver. J. Biol. Chem. 2006, 281, 28721-28730. [CrossRef] [PubMed] 
39. Iizuka, K. The Role of Carbohydrate Response Element Binding Protein in Intestinal and Hepatic Fructose Metabolism. Nutrients 2017, 9, 181. [CrossRef] [PubMed]

40. Iizuka, K.; Miller, B.; Uyeda, K. Deficiency of carbohydrate-activated transcription factor ChREBP prevents obesity and improves plasma glucose control in leptin-deficient (ob/ob) mice. Am. J. Physiol. Endocrinol. Metab. 2006, 291, E358-E364. [CrossRef] [PubMed]

41. Kim, M.; Astapova, I.I.; Flier, S.N.; Hannou, S.A.; Doridot, L.; Sargsyan, A.; Kou, H.H.; Fowler, A.J.; Liang, G.; Herman, M.A. Intestinal, but not hepatic, ChREBP is required for fructose tolerance. JCI Insight 2017, 2, e96703. [CrossRef] [PubMed]

42. Linden, A.G.; Li, S.; Choi, H.Y.; Fang, F.; Fukasawa, M.; Uyeda, K.; Hammer, R.E.; Horton, J.D.; Engelking, L.J.; Liang, G. Interplay between ChREBP and SREBP-1c Coordinates Postprandial Glycolysis and Lipogenesis in Livers of Mice. J. Lipid Res. 2018, 59, 475-487. [CrossRef] [PubMed]

43. Burgess, S.C.; lizuka, K.; Jeoung, N.H.; Harris, R.A.; Kashiwaya, Y.; Veech, R.L.; Kitazume, T.; Uyeda, K. Carbohydrate-response element-binding protein deletion alters substrate utilization producing an energy-deficient liver. J. Biol. Chem. 2008, 283, 1670-1678. [CrossRef] [PubMed]

44. Kim, J.K.; Lee, K.S.; Chang, H.Y.; Lee, W.K.; Lee, J.I. Progression of diet induced nonalcoholic steatohepatitis is accompanied by increased expression of kruppel-like-factor 10 in mice. J. Transl. Med. 2014, 12, 186. [CrossRef] [PubMed]

45. Kassam, A.; Capone, J.P.; Rachubinski, R.A. The short heterodimer partner receptor differentially modulates peroxisome proliferator-activated receptor alpha-mediated transcription from the peroxisome proliferator-response elements of the genes encoding the peroxisomal beta-oxidation enzymes acyl-CoA oxidase and hydratase-dehydrogenase. Mol. Cell. Endocrinol. 2001, 176, 49-56. [PubMed]

(c) 2018 by the authors. Licensee MDPI, Basel, Switzerland. This article is an open access article distributed under the terms and conditions of the Creative Commons Attribution (CC BY) license (http:/ / creativecommons.org/licenses/by/4.0/). 
Article

\title{
Folate and B12 Levels Correlate with Histological Severity in NASH Patients
}

\author{
Mahmud Mahamid ${ }^{1,2,+}$, Naim Mahroum ${ }^{3,+}$, Nicola Luigi Bragazzi ${ }^{4}$, Kasem Shalaata ${ }^{2,5}$, \\ Yarden Yavne ${ }^{3}$, Mohammad Adawi ${ }^{6}$ (D) ${ }^{\text {, Howard Amital }}{ }^{3}$ and Abdulla Watad ${ }^{3, *}$ (D) \\ 1 Endoscopy Unit, Nazareth Hospital EMMS, 16100 Nazareth, Israel; mahmudmahamid@yahoo.com \\ 2 Azrieli Faculty of Medicine, Bar-Ilan University, 13195 Safed, Israel; sh.kasem@hotmail.com \\ 3 Department of Medicine 'B', Sheba Medical Center, Tel-Hashomer, Israel, Sackler Faculty of Medicine, \\ Tel-Aviv University, 52621 Tel-Aviv, Israel; naim.mahroum@gmail.com (N.M.); \\ yavneyarden@gmail.com (Y.Y.); Howard.Amital@sheba.health.gov.il (H.A.) \\ 4 School of Public Health, Department of Health Sciences, University of Genoa, 16132 Genoa, Italy; \\ robertobragazzi@gmail.com \\ 5 Internal Medicine Department, Nazareth Hospital EMMS, 16100 Nazareth, Israel \\ 6 Ziv and Padeh Medical centers, 13195 Safed, Israel; Limak@012.net.il \\ * Correspondence: watad.abdulla@gmail.com; Tel.: +972-3-5302-652; Fax: +972-3-5304-796 \\ + These authors contributed equally to this work.
}

Received: 1 March 2018; Accepted: 30 March 2018; Published: 2 April 2018

\begin{abstract}
Background: The correlation between abnormal vitamin serum levels and chronic liver disease has been previously described in literature. However, the association between the severity of folate serum levels (B9), vitamin B12 and nonalcoholic steatohepatitis (NASH) has not been widely evaluated. Therefore, the aim of this study was to investigate the existence of such a correlation in a cohort of NASH patients. Methods: All patients aged 18 years and older who were diagnosed with biopsy-proven NASH at the EMMS hospital in Nazareth during the years 2015-2017 were enrolled in this study. Data regarding demographic, clinical and laboratory parameters was collected. Patients with other liver diseases were excluded. Results: Eighty-three NASH patients were enrolled during the study period. The mean age was $41 \pm 11$ years and the majority of patients were male. Mean values of folate and B12 were $9.85 \pm 10.90 \mathrm{ng} / \mathrm{mL}$ and $387.53 \pm 205.50 \mathrm{pg} / \mathrm{mL}$, respectively. Half of the patients were presented with a grade 1 steatosis (43.4\%), a grade 2 fibrosis $(50.6 \%)$ and a grade 3 activity score $(55.4 \%)$. The fibrosis grade was significantly correlated with low folate levels on multivariate analysis $(p$-value $<0.01)$. Similarly, low B12 levels were significantly associated with a higher fibrosis grade and NASH activity ( $p$-value $<0.001$ and $p$-value $<0.05$ respectively). Conclusion: Our study demonstrated a statistically significant correlation between low levels of folate and vitamin B12 with the histological severity of NASH. These findings could have diagnostic and therapeutic implications for patient management and follow-up.
\end{abstract}

Keywords: nonalcoholic fatty liver disease; NAFLD; nonalcoholic steatohepatitis; NASH; vitamin B9; folate; vitamin B12

\section{Introduction}

Nonalcoholic fatty liver disease (NAFLD) is defined as the presence of liver steatosis, as demonstrated on imaging or histology, in patients who ingest less than $20 \mathrm{mg}$ of alcohol per day $[1,2]$. It is the most prevalent liver disease worldwide, with an estimated global prevalence of $25.24 \%$ (95\% confidence interval (CI) 22.10-28.65), of which the highest rates are found in the Middle East and South America [3,4]. Different metabolic co-morbidities are often associated with NAFLD, including central obesity, insulin resistance, type 2 diabetes mellitus, hyperlipidemia, hypertension, and metabolic syndrome $[4,5]$. 
NAFLD is a wide spectrum of disorders ranging from a simple form of steatosis to a progressive form known as nonalcoholic steatohepatitis (NASH), which is characterized by hepatocellular injury and inflammation [6]. Furthermore, NAFLD is considered to be an important cause of cryptogenic cirrhosis [7]. According to a recently published meta-analysis, the mean annual rate of fibrosis progression in NASH is 0.09 (95\% CI 0.06-0.12), whilst the overall fibrosis progression proportion is $40.76 \%$ (95\% CI 34.69-47.13) [4]. Treatment of NASH continues to be challenging for physicians as there is no approved effective pharmacotherapy for this disorder and life style modification remains the mainstay of therapy for these patients $[2,8,9]$.

Vitamin deficiency/hypovitaminosis is a general term for a wide-spread condition which has reached epidemic proportions in developing and developed countries alike, mainly due to lifestyle and dietary habits. Although sub-clinical hypovitaminosis can occur in well-nourished subjects, it is most often the result of limited dietary intake or vitamin malabsorption $[9,10]$. Several studies have shown that chronic liver diseases are associated with lower levels of vitamins such as vitamin D, E, B6, and A [11-14]. Moreover, a trial therapeutic combination of vitamins E and A as a treatment for NASH was found to minimize oxidative stress damage and to curb cirrhosis formation processes [15].

Despite the indications in the literature regarding the association between chronic liver diseases and hypovitaminosis, little is known about a specific correlation between hypovitaminosis and NASH severity. Thus, in this study we sought to investigate potential correlations between folate and B12 serum levels with NASH severity, as defined in terms of the fibrosis grade and activity, in a geographical area characterized by a significant epidemiological burden of NASH. We chose to focus on these vitamins because the relationship between their serum levels and fibrosis severity has been scarcely reported. More specifically, we expected to find a negative correlation, in analogy with what was found for other vitamins.

\section{Material and Methods}

\subsection{Ethical Approval}

The current study received ethical approval from the local hospital ethical committee and was conducted according to the Helsinki declaration and its subsequent amendments. The data was coded in order to preserve the anonymity of the patients. Informed consent was waived because of the non-interventional design of the study.

\subsection{Patients Selection}

All patients diagnosed with biopsy-proven NASH at the EMMS Nazareth Hospital (located in Nazareth, Israel) between the years 2015 to 2017 were considered potentially eligible and enrolled in the study. Furthermore, patients were included in the present study if (i) age $>18$ years old; and (ii) they had no history of alcohol abuse (their weekly ethanol consumption was less than $210 \mathrm{gm}$ ).

Patients with other hepatic pathology or autoimmune phenotypes (such as alcoholic liver disease, drug-induced liver injury, autoimmune hepatitis, viral hepatitis, cholestatic liver disease and metabolic/genetic liver disease) were excluded using specific clinical, laboratory, radiological and/or histological criteria/tests (serology of viral hepatitis A, B, and C, autoimmune markers including ANA, anti-LKM, anti-smooth mussels protein electrophoresis, immune electrophoresis, metabolic markers such as serum ceruloplasmin, 24-h urine collection for copper, ferritin, iron, transferrin saturation, TSH, HbA1c and alpha-1 antitrypsin).

Extracted data included socio-demographic variables (such as age and gender), body mass index (BMI), fat volume, histopathological biopsy results, serum levels of alanine transaminase (ALT), aspartate transaminase (AST), low-density lipoprotein cholesterol (LDL), high-density lipoprotein cholesterol (HDL), triglycerides, insulin and insulin resistance index (HOMA-IR), glycated hemoglobin (hemoglobin A1c or HbA1c), C-reactive protein (CRP), folate, and vitamin B12. 
A hepatopathologist reviewed all of the liver biopsies and utilized the SAF scoring system (encompassing an assessment of steatosis (S), activity (A) and fibrosis (F)) scoring system for the grading and staging of steatohepatitis developed and validated by Bedossa and collaborators [16]. The staging, according to this scoring system, is as follows: steatosis: Stage 1 -lipid droplets in $\sim 30 \%$ of hepatocytes, Stage 2-steatosis in $60 \%$ of hepatocytes and Stage 3-steatosis in $>80 \%$ of hepatocytes; fibrosis: Stage 0 -no fibrosis, Stage 1-zone 3 perisinusoidal fibrosis, Stage 2-as before with portal fibrosis, Stage 3-as before with bridging fibrosis, and Stage 4-cirrhosis; activity score: A0—no activity, A1-mild activity, A2—moderate activity, A3 (A $\geq 3$ - - severe activity).

\subsection{Statistical Analysis}

Before commencing any statistical processing and analysis, the data was visually inspected and assessed for outliers. Continuous variables were computed as the mean with standard deviation (SD), whereas categorical variables were expressed as percentages. Univariate and multivariate regression analyses were conducted using the vitamin levels as dependent variables. Rank correlation analysis was performed; both Spearman's rho and Kendall's tau coefficients were computed. Spearman's rho coefficient was interpreted using the following rule of thumb (in absolute value): 0.00-0.19-very weak correlation, $0.20-0.39$ - weak correlation, 0.40-0.59-moderate correlation, 0.60-0.79-strong correlation, and 0.80-1.0-very strong correlation. Chi-squared test and analysis of variance (ANOVA)-one way were also performed.

All statistical analyses were performed with the commercial software Statistical Package for Social Science (SPSS version 24.0, IBM, Chicago, IL, USA). ROC analysis was performed with the commercial software MedCalc Statistical Software version 17.9.7 (MedCalc Software bvba, Ostend, Belgium; http:/ / www.medcalc.org; 2017). A $p$-value of less than 0.05 was considered statistically significant.

\section{Results}

Eighty-three NASH patients were enrolled in this study. The mean age of the study population was $41.12(\mathrm{SD} \pm 11.18$ ) years. The majority of participants were male $(61.4 \%)$. The mean serum levels of folate and vitamin B12 amongst the study population were $9.85 \pm 10.90 \mathrm{ng} / \mathrm{mL}$ and $387.53 \pm 205.50 \mathrm{pg} / \mathrm{mL}$, respectively. Roughly half of the patients were presented with a grade 1 steatosis $(43.4 \%)$, grade 2 fibrosis $(50.6 \%$ ) and a grade 3 activity score $(55.4 \%)$. Further details regarding the study population can be seen in Table 1 .

Table 1. Descriptive statistics of the population studied.

\begin{tabular}{cc}
\hline Variables & Values \\
\hline Age (years) & $41.12 \pm 11.18 ; 42(18-74)$ \\
\hline Gender & $51(61.4 \%)$ \\
\hline Male & $32(38.6 \%)$ \\
Female & $28.86 \pm 2.83 ; 29(22-40)$ \\
BMI $\left(\mathrm{kg} / \mathrm{m}^{2}\right)$ & $55.66 \pm 24.60 ; 60(10-90)$ \\
Fat volume & $80.61 \pm 31.33 ; 78(11-212)$ \\
ALT (units) & $48.81 \pm 14.99 ; 45(24-85)$ \\
AST (units) & $3.80 \pm 2.44 ; 3.6(0-13)$ \\
CRP (mg/dL) & $37.69 \pm 8.63 ; 36(16-58)$ \\
HDL (mg/dL) & $132.36 \pm 24.01 ; 139(68-187)$ \\
LDL (mg/dL) & $153.12 \pm 36.63 ; 152(69-274)$ \\
TG (mg/dL) & $24.71 \pm 5.21 ; 26(12-36.2)$ \\
Insulin $(\mathrm{IU} / \mathrm{ML})$ & $5.38 \pm 0.63 ; 5.4(2-6.2)$ \\
HbA1c $(\%)$ & $2.88 \pm 1.06 ; 2.8(1.2-5.9)$ \\
HOMA-IR & $387.53 \pm 205.50 ; 321(123-823)$ \\
B12 (pg/mL) & $9.85 \pm 10.90 ; 8.9(2.2-105)$ \\
Folate (ng/mL) &
\end{tabular}


Table 1. Cont.

\begin{tabular}{cc}
\hline Variables & Values \\
\hline Steatosis (S0-S3) & \\
\hline S1 & $36(43.4 \%)$ \\
S2 & $24(28.9 \%)$ \\
S3 & $23(27.7 \%)$ \\
\hline Fibrosis (F0-F4) & \\
\hline Grade 0 & $11(13.3 \%)$ \\
Grade 1 & $20(24.1 \%)$ \\
Grade 2 & $42(50.6 \%)$ \\
Grade 3 & $7(8.4 \%)$ \\
Grade 4 & $3(3.6 \%)$ \\
\hline Activity (A0-A4) & \\
\hline Grade 2 & $36(43.4 \%)$ \\
Grade 3 & $46(55.4 \%)$ \\
Grade 4 & $1(1.2 \%)$ \\
\hline
\end{tabular}

Abbreviations: BMI, body mass index; ALT, alanine transaminase; AST, aspartate aminotransferase; CRP, C-reactive protein; HDL, high-density lipoproteins; LDL, low-density lipoproteins; TG, triglycerides; HOMA-IR, homeostatic model assessment-Insulin resistance (HOMA-IR).

A statistically significant association was found between lower levels of folate and fibrosis grade on univariate regression analysis $(p$-value $<0.01$ ). Older age, CRP levels, HOMA-IR, fibrosis grade and NASH disease activity grade were all significantly correlated with lower levels of vitamin B12 (Table 2).

Table 2. Univariate regression analysis for each vitamin studied in the present investigation.

\begin{tabular}{ccc}
\hline Variable & Folate $(\mathrm{ng} / \mathrm{mL})$ & B12 $(\mathrm{pg} / \mathrm{mL})$ \\
\hline Age (years) & $0.002 \pm 0.108$ & $-4.846 \pm 1.971^{*}$ \\
Gender & $2.730 \pm 2.454$ & $-2.138 \pm 46.629$ \\
BMI (kg/m $\left.{ }^{2}\right)$ & $0.085 \pm 0.428$ & $0.764 \pm 8.062$ \\
Fat volume & $0.008 \pm 0.049$ & $0.755 \pm 0.924$ \\
ALT (units) & $-0.034 \pm 0.038$ & $-1.291 \pm 0.715$ \\
AST (units) & $-0.090 \pm 0.080$ & $-1.972 \pm 1.507$ \\
CRP (mg/dL) & $-0.056 \pm 0.500$ & $-20.007 \pm 9.072 *$ \\
HDL (mg/dL) & $-0.105 \pm 0.140$ & $3.765 \pm 2.612$ \\
LDL (mg/dL) & $0.077 \pm 0.050$ & $-0.136 \pm 0.951$ \\
TG (mg/dL) & $0.032 \pm 0.033$ & $-0.722 \pm 0.618$ \\
Insulin (IU/mL) & $-0.431 \pm 0.227$ & $-7.564 \pm 4.300$ \\
HbA1c (\%) & $-1.004 \pm 1.908$ & $-62.653 \pm 35.350$ \\
HOMA-IR & $-1.714 \pm 1.127$ & $-45.663 \pm 20.947^{*}$ \\
Steatosis & $-0.773 \pm 1.450$ & $30.421 \pm 27.186$ \\
Fibrosis & $-3.665 \pm 1.218^{* *}$ & $-160.835 \pm 16.351 * * *$ \\
Activity & $-3.196 \pm 2.298$ & $-131.579 \pm 41.334^{* *}$
\end{tabular}

Abbreviations: BMI, body mass index; ALT, alanine transaminase; AST, aspartate aminotransferase; CRP, C-reactive protein; HDL, high-density lipoproteins; LDL, low-density lipoproteins; TG, triglycerides; HOMA-IR, homeostatic model assessment-insulin resistance (HOMA-IR). * statistically significant with $p$-value $<0.05 ;{ }^{* *}$ statistically significant with $p$-value $<0.01 ;{ }^{* *}$ statistically significant with $p$-value $<0.001$.

When confounders such as obesity and insulin resistance were taken into account on multivariate analysis, fibrosis was found to be significantly associated with folate serum levels (standardized beta coefficient $-0.353, p=0.009$ ). On rank correlation analysis, the link between folate levels and fibrosis grade was of statistical significance (Spearman's rho -0.608 (95\%CI from -0.728 to $-0.451)$, $p$-value $<0.001$, strong correlation; Kendall's tau -0.487 (95\%CI from -0.603 to -0.335 ), $p$-value $<0.001)$. Fibrosis was also significantly correlated with vitamin B12 levels, on both the regression (standardized beta coefficient $-0.623, p$-value $=0.000$ ) and on rank correlation analysis (Spearman's rho -0.737 (95\%CI from -0.822 to -0.620$)$, $p$-value $<0.001$, strong correlation; Kendall's tau -0.606 ( $(95 \% \mathrm{CI}$ from -0.688 to -0.489$), p$-value $<0.001)$. Activity was also associated with vitamin B12 levels (standardized beta coefficient $-0.183, p$-value $=0.039$ ), even though the correlation found 
was very weak (Spearman's rho -0.288 [95\%CI from -0.474 to -0.0770 ), $p$-value $=0.0083$; Kendall's tau $-0.237(95 \%$ CI from -0.416 to -0.020$), p$-value $=0.0015)$. The analyses are presented in Table 3.

Table 3. Multivariate regressions for each vitamin.

\begin{tabular}{|c|c|c|c|c|c|}
\hline \multirow{2}{*}{ Variables } & \multicolumn{2}{|c|}{ Non-Standardized Coefficients } & \multirow{2}{*}{$\begin{array}{l}\text { Standardized Coefficients } \\
\text { Beta }\end{array}$} & \multirow{2}{*}{$\mathrm{T}$} & \multirow{2}{*}{ Sig. } \\
\hline & B & Standard Deviation & & & \\
\hline \multicolumn{6}{|c|}{$\begin{array}{ll}\text { Folate (B9) } \\
\end{array}$} \\
\hline (Constant) & 6.959 & 21.122 & & 0.329 & 0.743 \\
\hline Age (years) & 0.188 & 0.128 & 0.193 & 1.469 & 0.147 \\
\hline Gender & 5.101 & 2.710 & 0.229 & 1.882 & 0.064 \\
\hline BMI $\left(\mathrm{kg} / \mathrm{m}^{2}\right)$ & 0.382 & 0.480 & 0.099 & 0.795 & 0.429 \\
\hline AST (units) & -0.087 & 0.095 & -0.119 & -0.914 & 0.364 \\
\hline ALT (units) & -0.003 & 0.107 & -0.004 & -0.031 & 0.975 \\
\hline $\mathrm{TG}(\mathrm{mg} / \mathrm{dL})$ & 0.024 & 0.035 & 0.079 & 0.680 & 0.499 \\
\hline $\operatorname{HDL}(\mathrm{mg} / \mathrm{dL})$ & -0.147 & 0.148 & -0.116 & -0.996 & 0.323 \\
\hline $\mathrm{LDL}(\mathrm{mg} / \mathrm{dL})$ & 0.034 & 0.053 & 0.074 & 0.637 & 0.526 \\
\hline $\mathrm{CRP}(\mathrm{mg} / \mathrm{dL})$ & 0.519 & 0.567 & 0.116 & 0.915 & 0.364 \\
\hline $\mathrm{HbA} 1 \mathrm{c}(\%)$ & -0.065 & 2.113 & -0.004 & -0.031 & 0.976 \\
\hline Insulin (IU/mL) & -0.304 & 0.324 & -0.145 & -0.938 & 0.352 \\
\hline HOMA-IR & -0.742 & 1.697 & -0.072 & -0.438 & 0.663 \\
\hline Fibrosis & -4.079 & 1.513 & -0.353 & -2.695 & $0.009^{* *}$ \\
\hline Activity & -0.595 & 2.575 & -0.028 & -0.231 & 0.818 \\
\hline Steatosis & -6.073 & 3.461 & -0.464 & -1.755 & 0.084 \\
\hline Fat volume & 0.188 & 0.120 & 0.424 & 1.566 & 0.122 \\
\hline \multicolumn{6}{|c|}{ Vitamin B12 } \\
\hline (Constant) & 1264.063 & 280.796 & & 4.502 & $0.000 * * *$ \\
\hline Age (years) & -0.814 & 1.705 & -0.044 & -0.478 & 0.634 \\
\hline Gender & -9.612 & 36.033 & -0.023 & -0.267 & 0.790 \\
\hline BMI $\left(\mathrm{kg} / \mathrm{m}^{2}\right)$ & -5.805 & 6.384 & -0.080 & -0.909 & 0.366 \\
\hline AST (units) & -0.586 & 1.260 & -0.043 & -0.465 & 0.643 \\
\hline ALT (units) & -2.413 & 1.416 & -0.137 & -1.704 & 0.093 \\
\hline $\mathrm{TG}(\mathrm{mg} / \mathrm{dL})$ & -0.451 & 0.460 & -0.080 & -0.981 & 0.330 \\
\hline $\operatorname{HDL}(\mathrm{mg} / \mathrm{dL})$ & 3.060 & 1.961 & 0.129 & 1.561 & 0.123 \\
\hline $\mathrm{LDL}(\mathrm{mg} / \mathrm{dL}$ & 0.547 & 0.702 & 0.064 & 0.780 & 0.438 \\
\hline $\mathrm{CRP}(\mathrm{mg} / \mathrm{dL})$ & -5.564 & 7.537 & -0.066 & -0.738 & 0.463 \\
\hline $\mathrm{HbA1c}(\%)$ & -31.261 & 28.088 & -0.096 & -1.113 & 0.270 \\
\hline Insulin (IU/mL) & 1.503 & 4.304 & 0.038 & 0.349 & 0.728 \\
\hline HOMA-IR & -4.149 & 22.558 & -0.021 & -0.184 & 0.855 \\
\hline Fibrosis & -135.886 & 20.118 & -0.623 & -6.754 & $0.000 * * *$ \\
\hline Activity & -72.172 & 34.236 & -0.183 & -2.108 & 0.039 * \\
\hline Steatosis & 66.491 & 46.012 & 0.270 & 1.445 & 0.153 \\
\hline Fat volume & -1.966 & 1.594 & -0.235 & -1.233 & 0.222 \\
\hline
\end{tabular}

Abbreviations: BMI, body mass index; ALT, alanine transaminase; AST, aspartate aminotransferase; CRP, C-reactive protein; HDL, high-density lipoproteins; LDL, low-density lipoproteins; TG, triglycerides; HOMA-IR, homeostatic model assessment-Insulin resistance (HOMA-IR). ${ }^{*}$ statistically significant with $p$-value $<0.05$; ${ }^{*}$ statistically significant with $p$-value $<0.01 ;{ }^{* * *}$ statistically significant with $p$-value $<0.001$.

\section{Discussion}

Information regarding the relationship between folate and vitamin B12 status and the histological severity of NASH is scarce in the literature. In this study, both folate and vitamin B12 serum levels were found to be significantly correlated with the liver fibrosis grade, whereas vitamin B12 status was also associated with NASH degree of activity.

To date, the correlation between vitamin levels and chronic liver diseases has been investigated in several studies, although most of the data focused on vitamin D status [17-19]. Dasarathy et al. [20] compared plasma vitamin D concentration levels between 81 NAFLD/NASH patients, 67 individuals with hepatic steatosis and 39 healthy controls. Vitamin D serum levels were found to be significantly lower in NAFLD/NASH patients, with a negative correlation between vitamin D status and NAFLD activity scores. Low concentrations of vitamin D were additionally associated with greater severity of steatosis, hepatocyte ballooning, and fibrosis, although only the association with the severity of hepatocyte ballooning remained statistically significant after it was adjusted for confounders. Furthermore, plasma vitamin D and insulin concentrations were independent predictors of the NAFLD activity score on biopsy, while a higher fat mass was found to correlate with the low vitamin D levels 
in NAFLD patients. An additional study, which examined 46 morbidly obese patients with diabetes mellitus and metabolic syndrome, of which $72 \%$ had NASH [21], discovered a relationship between a higher fibrosis stage and higher levels of HOMA-IR and vitamin D. On the other hand, according to a study by Brill and colleagues [22], although subjects with NASH had higher insulin resistance than individuals without NASH, plasma vitamin D concentration was similar between both groups. However, despite these controversies concerning the role of vitamin D in NASH, other vitamins, such as folate and vitamin B12, have been overlooked in the extant literature.

Various studies have demonstrated that obese subjects have lower folate serum levels in comparison with normal-range BMI subjects $[23,24]$. Furthermore, obesity is a well-known risk factor for NAFLD [25]. Therefore, it has been suggested that folate deficiency may have a pathogenic role in NAFLD. This leads to a more severe form of the disease, mainly in metabolic syndrome and diabetes mellitus type 2 patients [26]. Moreover, Sid et al. [26] highlighted the importance of folate in the progression of NAFLD, thus raising the possibility that supplementary folate may be a treatment option. These findings are consistent with our results, in which a correlation between lower folate levels and a higher histological grading of NASH was revealed.

In a cohort of Chinese patients with NAFLD, low serum concentration of folate was identified as an independent risk factor for NAFLD [27]. Furthermore, it was demonstrated that inclusion of serum folate levels in the current NAFLD prediction score has led to a significant improvement in NAFLD prediction [27]. However, in an open-label pilot study [28], ten NASH patients with a median fibrosis stage 2 were treated with $1 \mathrm{mg}$ /day of folic acid for 6 months. No significant effects in terms of biochemical improvement could be detected. Furthermore, folate deficiency was observed only in a cirrhotic patient. Therefore, it is clear that the controversy in the literature regarding the complex interplay between folate and non-alcoholic fatty liver disease and steatohepatitis has yet to be resolved. However, unveiling the pathogenetic process underlying the association between folate and NAFLD may provide important insights into the diagnosis and prediction of NASH, thus enabling treatment initiation in a timely and effective manner.

Another important finding of this study was the role of low vitamin B12 levels as an independent predictor of NASH histological severity, in terms of disease activity and fibrosis grade. These results corroborated the outcomes of previous studies, which, for instance, demonstrated that NAFLD patients have lower serum levels of vitamin B12 in comparison to controls, which correlated with a higher grade of steatohepatitis [29]. In another study [30], higher levels of homocysteine were observed in 71 NAFLD patients, which correlated negatively with folate and B12 concentrations.

However, in a study by Polyzos et al. [31], no difference in serum vitamin B12 and folate levels was detected in 30 NAFLD patients in comparison with 24 healthy controls. Additionally, no correlation was found between the levels of both vitamins and the severity of liver disease, in terms of steatosis grade and fibrosis stage. These results were in contrast with the findings of another study which reported that B12 could be used as a biomarker in a sample of 116 patients with chronic hepatitis C [32]. Serum B12 levels positively correlated with AST, ALT, baseline viral load, stage of fibrosis, and favorable interferon- $\lambda 3 / 4$ rs12979860 genotypes. However, they were found to be inversely correlated with sustained virological response, as well as with rapid virological response.

Moreover, Goel et al. [33] found higher serum levels of vitamin B12 in patients with cryptogenic cirrhosis when compared to patients with idiopathic non-cirrhotic intrahepatic portal hypertension (NCIPH), therefore suggesting that B12 levels may be used as a differentiating marker between the two pathologies. Similarly, levels of vitamin B12 were also found to be significantly higher in patients with alcoholic liver cirrhosis than in matching healthy individuals [34]. Nevertheless, when taking into account the relevance of these results to the findings in our study, it should be noted that the majority $(96 \%)$ of NASH patients in our study did not have cirrhosis. Therefore, it could be surmised that while low levels of vitamin B12 may predict the development of a severe form of NASH, high levels may indicate the commencement of end stage liver disease. 
The relationship between serum vitamin B12 levels and insulin resistance has been widely addressed in the literature, yet remains controversial. Our study found that levels of vitamin B12 are inversely correlated with HOMA-IR, which is a known risk factor for NAFLD development [35]. This illustrates the probability that low serum levels of vitamin B12 might play a pathogenic part in NAFLD development. Similar findings regarding the ability of low levels of vitamin B12 and folate to predict insulin resistance were reported by Li et al. [36], however it should be noted that this study was conducted on obese subjects. Despite these compelling results, other studies did not detect a link between low vitamin B12 levels and insulin resistance or metabolic syndrome, whereas a correlation was found with obesity and being overweight [37]. Gammon et al. [38] reached an identical conclusion when assessing the relationship between insulin resistance and vitamin B12 levels in a vegetarian population.

Our study has several limitations which ought to be properly addressed. The major drawback is the cross-sectional study design which, due to its very nature, does not enable the deduction of causal inferences with regard to the relationship between vitamin status and NASH in terms of development and pathophysiology. Another limitation is the lack of data concerning patient co-morbidities and drugs regimens.

\section{Conclusions}

NASH is a chronic liver condition which imposes a dramatic burden, both in epidemiological and clinical terms. Our study demonstrated that low levels of folate (B9) and vitamin B12 can be used as independent predictors of the histological severity of NASH. Thus, these findings may have practical implications in the follow-up and prognosis assessment of NASH patients. Further studies regarding the pathological role of low folate and B12 levels in NASH development are warranted.

Author Contributions: M.M., N.M. and A.W. directed the study; M.M., A.W. and H.A. conceived and designed the study; M.M. and K.S. collected the data; N.L.B. analyzed the data; K.S., Y.Y., M.A. and N.M. wrote the paper.

Conflicts of Interest: The authors declare no conflict of interest.

\section{References}

1. Abd El-Kader, S.M.; El-Den Ashmawy, E.M.S. Non-alcoholic fatty liver disease: The diagnosis and management. World J. Hepatol. 2015, 7, 846-858. [CrossRef] [PubMed]

2. Tolman, K.G.; Dalpiaz, A.S. Treatment of non-alcoholic fatty liver disease. Ther. Clin. Risk Manag. 2007, 3, 1153-1163. [PubMed]

3. Bedossa, P. Pathology of non-alcoholic fatty liver disease. Liver Int. Off. J. Int. Assoc. Study Liver 2017, 37, 85-89. [CrossRef] [PubMed]

4. Younossi, Z.M.; Koenig, A.B.; Abdelatif, D.; Fazel, Y.; Henry, L.; Wymer, M. Global epidemiology of nonalcoholic fatty liver disease-Meta-analytic assessment of prevalence, incidence, and outcomes. Hepatology 2016, 64, 73-84. [CrossRef] [PubMed]

5. Milic, S.; Mikolasevic, I.; Krznaric-Zrnic, I.; Stanic, M.; Poropat, G.; Stimac, D.; Vlahovic-Palcevski, V.; Orlic, L. Nonalcoholic steatohepatitis: Emerging targeted therapies to optimize treatment options. Drug Des. Dev. Ther. 2015, 9, 4835-4845. [CrossRef] [PubMed]

6. Takahashi, Y.; Fukusato, T. Histopathology of nonalcoholic fatty liver disease/nonalcoholic steatohepatitis. World J. Gastroenterol. 2014, 20, 15539-15548. [CrossRef] [PubMed]

7. Aithal, G.P.; Ramsay, L.; Daly, A.K.; Sonchit, N.; Leathart, J.B.; Alexander, G.; Kenna, J.G.; Caldwell, J.; Day, C.P. Hepatic adducts, circulating antibodies, and cytokine polymorphisms in patients with diclofenac hepatotoxicity. Hepatology 2004, 39, 1430-1440. [CrossRef] [PubMed]

8. Hardy, T.; Anstee, Q.M.; Day, C.P. Nonalcoholic fatty liver disease: New treatments. Curr. Opin. Gastroenterol. 2015, 31, 175-183. [CrossRef] [PubMed]

9. Barchetta, I.; Cimini, F.A.; Cavallo, M.G. Vitamin D Supplementation and Non-Alcoholic Fatty Liver Disease: Present and Future. Nutrients 2017, 9, 1015. [CrossRef] [PubMed] 
10. McKinney, T.J.; Patel, J.J.; Benns, M.V.; Nash, N.A.; Miller, K.R. Vitamin D Status and supplementation in the critically Ill. Curr. Gastroenterol. Rep. 2016, 18, 18. [CrossRef] [PubMed]

11. Stokes, C.S.; Volmer, D.A.; Grunhage, F.; Lammert, F. Vitamin D in chronic liver disease. Liver Int. Off. J. Int. Assoc. Study Liver 2013, 33, 338-352. [CrossRef] [PubMed]

12. Di Sario, A.; Candelaresi, C.; Omenetti, A.; Benedetti, A. Vitamin E in chronic liver diseases and liver fibrosis. Vitam. Horm. 2007, 76, 551-573. [PubMed]

13. Rossouw, J.E.; Labadarios, D.; McConnell, J.B.; Davis, M.; Williams, R. Plasma pyridoxal phosphate levels in fulminant hepatic failure and the effects of parenteral supplementation. Scand. J. Gastroenterol. 1977, 12, 123-127. [PubMed]

14. Venu, M.; Martin, E.; Saeian, K.; Gawrieh, S. High prevalence of Vitamin A deficiency and Vitamin D deficiency in patients evaluated for liver transplantation. Liver Transplant. 2013, 19, 627-633. [CrossRef] [PubMed]

15. Kawanaka, M.; Nishino, K.; Nakamura, J.; Suehiro, M.; Goto, D.; Urata, N.; Oka, T.; Kawamoto, H.; Nakamura, H.; Yodoi, J.; et al. Treatment of nonalcoholic steatohepatitis with Vitamins E and C: A pilot study. Hepatic Med. Evid. Res. 2013, 5, 11-16. [CrossRef] [PubMed]

16. Bedossa, P.; Poitou, C.; Veyrie, N.; Bouillot, J.L.; Basdevant, A.; Paradis, V.; Tordjman, J.; Clement, K. Histopathological algorithm and scoring system for evaluation of liver lesions in morbidly obese patients. Hepatology 2012, 56, 1751-1759. [CrossRef] [PubMed]

17. Iruzubieta, P.; Terán, Á.; Crespo, J.; Fábrega, E. Vitamin D deficiency in chronic liver disease. World J. Hepatol. 2014, 6, 901-915. [CrossRef] [PubMed]

18. Chen, E.-Q.; Shi, Y.; Tang, H. New insight of Vitamin D in chronic liver diseases. Hepatobiliary Pancreat. Dis. Int. 2014, 13, 580-585. [CrossRef]

19. Chaves, G.V.; Peres, W.A.; Goncalves, J.C.; Ramalho, A. Vitamin A and retinol-binding protein deficiency among chronic liver disease patients. Nutrition 2015, 31, 664-668. [CrossRef] [PubMed]

20. Dasarathy, J.; Periyalwar, P.; Allampati, S.; Bhinder, V.; Hawkins, C.; Brandt, P.; Khiyami, A.; McCullough, A.J.; Dasarathy, S. Hypovitaminosis D is associated with increased whole body fat mass and greater severity of non-alcoholic fatty liver disease. Liver Int. Off. J. Int. Assoc. Study Liver 2014, 34, e118-e127. [CrossRef] [PubMed]

21. Luger, M.; Kruschitz, R.; Kienbacher, C.; Traussnigg, S.; Langer, F.B.; Schindler, K.; Wurger, T.; Wrba, F.; Trauner, M.; Prager, G.; et al. Prevalence of Liver Fibrosis and its Association with Non-invasive Fibrosis and Metabolic Markers in Morbidly Obese Patients with Vitamin D Deficiency. Obes. Surg. 2016, 26, 2425-2432. [CrossRef] [PubMed]

22. Bril, F.; Maximos, M.; Portillo-Sanchez, P.; Biernacki, D.; Lomonaco, R.; Subbarayan, S.; Correa, M.; Lo, M.; Suman, A.; Cusi, K. Relationship of Vitamin D with insulin resistance and disease severity in non-alcoholic steatohepatitis. J. Hepatol. 2015, 62, 405-411. [CrossRef] [PubMed]

23. Casanueva, E.; Drijanski, A.; Fernández-Gaxiola, A.C.; Meza, C.; Pfeffer, F. Folate deficiency is associated with obesity and anemia in Mexican urban women. Nutr. Res. 2000, 20, 1389-1394. [CrossRef]

24. Hirsch, S.; Poniachick, J.; Avendano, M.; Csendes, A.; Burdiles, P.; Smok, G.; Diaz, J.C.; de la Maza, M.P. Serum folate and homocysteine levels in obese females with non-alcoholic fatty liver. Nutrition 2005, 21, 137-141. [CrossRef] [PubMed]

25. Fabbrini, E.; Sullivan, S.; Klein, S. Obesity and Nonalcoholic Fatty Liver Disease: Biochemical, Metabolic and Clinical Implications. Hepatology 2010, 51, 679-689. [CrossRef] [PubMed]

26. Sid, V.; Siow, Y.L.; O, K. Role of folate in nonalcoholic fatty liver disease. Can. J. Physiol. Pharmacol. 2017, 95, 1141-1148. [CrossRef] [PubMed]

27. Xia, M.F.; Bian, H.; Zhu, X.P.; Yan, H.M.; Chang, X.X.; Zhang, L.S.; Lin, H.D.; Hu, X.Q.; Gao, X. Serum folic acid levels are associated with the presence and severity of liver steatosis in Chinese adults. Clin. Nutr. 2017. [CrossRef] [PubMed]

28. Charatcharoenwitthaya, P.; Levy, C.; Angulo, P.; Keach, J.; Jorgensen, R.; Lindor, K.D. Open-label pilot study of folic acid in patients with nonalcoholic steatohepatitis. Liver Int. Off. J Int. Assoc. Study Liver 2007, 27, 220-226. [CrossRef] [PubMed]

29. Koplay, M.; Gulcan, E.; Ozkan, F. Association between serum Vitamin B12 levels and the degree of steatosis in patients with nonalcoholic fatty liver disease. J. Investig. Med. 2011, 59, 1137-1140. [CrossRef] [PubMed] 
30. Gulsen, M.; Yesilova, Z.; Bagci, S.; Uygun, A.; Ozcan, A.; Ercin, C.N.; Erdil, A.; Sanisoglu, S.Y.; Cakir, E.; Ates, Y.; et al. Elevated plasma homocysteine concentrations as a predictor of steatohepatitis in patients with non-alcoholic fatty liver disease. J. Gastroenterol. Hepatol. 2005, 20, 1448-1455. [CrossRef] [PubMed]

31. Polyzos, S.A.; Kountouras, J.; Patsiaoura, K.; Katsiki, E.; Zafeiriadou, E.; Zavos, C.; Deretzi, G.; Tsiaousi, E.; Slavakis, A. Serum Vitamin B12 and folate levels in patients with non-alcoholic fatty liver disease. Int. J. Food Sci. Nutr. 2012, 63, 659-666. [CrossRef] [PubMed]

32. Mechie, N.C.; Goralzcyk, A.D.; Reinhardt, L.; Mihm, S.; Amanzada, A. Association of serum Vitamin B12 levels with stage of liver fibrosis and treatment outcome in patients with chronic hepatitis $C$ virus genotype 1 infection: A retrospective study. BMC Res. Notes 2015, 8, 260. [CrossRef] [PubMed]

33. Goel, A.; Ramakrishna, B.; Muliyil, J.; Madhu, K.; Sajith, K.G.; Zachariah, U.; Ramachandran, J.; Keshava, S.N.; Selvakumar, R.; Chandy, G.M.; et al. Use of serum Vitamin B12 level as a marker to differentiate idiopathic noncirrhotic intrahepatic portal hypertension from cryptogenic cirrhosis. Dig. Dis. Sci. 2013, 58, 179-187. [CrossRef] [PubMed]

34. Kazimierska, E.; Czestochowska, E. Serum homocysteine, Vitamin B12 and folic acid concentrations in patients with alcoholic liver cirrhosis. Polski Merkur. Lek. 2003, 15, 140-143.

35. Gaggini, M.; Morelli, M.; Buzzigoli, E.; DeFronzo, R.A.; Bugianesi, E.; Gastaldelli, A. Non-Alcoholic Fatty Liver Disease (NAFLD) and Its Connection with Insulin Resistance, Dyslipidemia, Atherosclerosis and Coronary Heart Disease. Nutrients 2013, 5, 1544-1560. [CrossRef] [PubMed]

36. Li, Z.; Gueant-Rodriguez, R.M.; Quilliot, D.; Sirveaux, M.A.; Meyre, D.; Gueant, J.L.; Brunaud, L. Folate and Vitamin B12 status is associated with insulin resistance and metabolic syndrome in morbid obesity. Clin. Nutr. 2017. [CrossRef] [PubMed]

37. Baltaci, D.; Kutlucan, A.; Turker, Y.; Yilmaz, A.; Karacam, S.; Deler, H.; Ucgun, T.; Kara, I.H. Association of Vitamin B12 with obesity, overweight, insulin resistance and metabolic syndrome, and body fat composition; primary care-based study. Med. Glas. 2013, 10, 203-210.

38. Gammon, C.S.; von Hurst, P.R.; Coad, J.; Kruger, R.; Stonehouse, W. Vegetarianism, Vitamin B12 status, and insulin resistance in a group of predominantly overweight/obese South Asian women. Nutrition 2012, 28, 20-24. [CrossRef] [PubMed]

(C) 2018 by the authors. Licensee MDPI, Basel, Switzerland. This article is an open access article distributed under the terms and conditions of the Creative Commons Attribution (CC BY) license (http:/ / creativecommons.org/licenses/by/4.0/). 
Article

\title{
Circulating Phospholipid Patterns in NAFLD Patients Associated with a Combination of Metabolic Risk Factors
}

\author{
Shilpa Tiwari-Heckler, Hongying Gan-Schreier, Wolfgang Stremmel, Walee Chamulitrat and \\ Anita Pathil * (iD \\ Department of Internal Medicine IV, Gastroenterology and Hepatology, University of Heidelberg, \\ Im Neuenheimer Feld 410, 69120 Heidelberg, Germany; Shilpa.Tiwari-Heckler@med.uni-heidelberg.de (S.T.-H.); \\ Hongying.Gan-Schreier@med.uni-heidelberg.de (H.G.-S.); Wolfgang.Stremmel@med.uni-heidelberg.de (W.S.); \\ Walee.Chamulitrat@med.uni-heidelberg.de (W.C.) \\ * Correspondence: Anita.Pathil-Warth@med.uni-heidelberg.de; Tel.: +49-6221-5638102
}

Received: 10 April 2018; Accepted: 15 May 2018; Published: 21 May 2018

\begin{abstract}
Background: Non-alcoholic fatty liver disease (NAFLD) is associated with inefficient macroand micronutrient metabolism, and alteration of circulating phospholipid compositions defines the signature of NAFLD. This current study aimed to assess the pattern of serum phospholipids in the spectrum of NAFLD, and its related comorbidities and genetic modifications. Methods: 97 patients with diagnosed NAFLD were recruited at a single center during 2013-2016. Based on histological and transient elastography assessment, 69 patients were divided into non-alcoholic steatohepatitis (NASH) and non-alcoholic fatty liver (NAFL) subgroups. 28 patients served as healthy controls. Serum phospholipids were determined by liquid-chromatography mass spectrometry (LC-MS/MS). Results: The total content of phosphatidylcholine (PC) and sphingomyelin in the serum was significantly increased in NAFL and NASH patients, compared to healthy controls. In addition, serum lysophospatidylethanolamine levels were significantly decreased in NAFL and NASH individuals. Circulating PC species, containing linoleic and $\alpha$-linolenic acids, were markedly increased in NAFLD patients with hypertension, compared to NAFLD patients without hypertension. The pattern of phospholipids did not differ between NAFLD patients with diabetes and those without diabetes. However, NAFLD patients with hyperglycemia (blood glucose level (BGL) $>100 \mathrm{mg} / \mathrm{dL}$ ) exhibited significantly a higher amount of monounsaturated phosphatidylethanolamine than those with low blood glucose levels. In addition, NAFLD patients with proven GG-genotype of PNPLA3, who were at higher risk for the development of progressive disease with fibrosis, showed lower levels of circulating plasmalogens, especially 16:0, compared to those with CC- and CG-allele. Conclusions: Our extended lipidomic study presents a unique metabolic profile of circulating phospholipids associated with the presence of metabolic risk factors or the genetic background of NAFLD patients.
\end{abstract}

Keywords: non-alcoholic fatty liver disease; NAFLD; phospholipids; metabolic disease

\section{Introduction}

Non-alcoholic fatty liver disease (NAFLD) is one of the most common causes of chronic liver disease in the western world, and is linked to increasing prevalence of obesity and diabetes [1,2]. One of the major contributors to this issue is overconsumption with an inefficient metabolism of macro- and micronutrients, affecting immune response and consequently leading to chronic liver disease [3]. NAFLD extends from lipid accumulation in the liver (non-alcoholic fatty liver (NAFL)) to non-alcoholic steatohepatitis (NASH), which may progress to liver cirrhosis and end-stage liver disease $[4,5]$. Disturbed hepatic lipid metabolism is a hallmark of NAFLD. Certain lipids, such as 
diacylglycerol, free fatty acids and ceramides, seem to mediate inflammatory pathways leading to lipotoxicity and oxidative stress, which finally may contribute to disease progression [6]. Previous metabolic studies have provided new insights into altered phospholipid metabolism in NAFLD, implicating an important pathophysiological role for this lipid class [7-9]. Puri et al. showed a significant decrease of hepatic phosphatidiylcholine (PC) levels and hepatic lipid subclasses containing arachidonic acid (AA) in NAFLD patients [7]. The progression of NAFL to NASH is characterized by an altered monounsaturated (MUFA) and $n 3$ - and $n 6$-polyunsaturated (PUFA) metabolism, probably due to deranged activities of $\delta-5,-6$, and -9 desaturases, as well as by an impaired PUFA oxidation process, leading to an increase in the total plasma content of non-enzymatic autoxidation products of PUFA lipids, e.g. 11-Hydroxyeicosatetraenoic acid (11-HETE) [7,10], 9-Hydroxyoctadecadienoic acid (9-HODE) and 13-Hydroxyoctadecadienoic acid (13-HODE) [11] or 20-carboxy arachidonic acid (20-COOH AA) [12]. NAFLD is associated with different clinical manifestations of the metabolic syndrome, including obesity, diabetes and hypertension [2,5]. Development of hepatic steatosis and progression to NASH is also linked to genetic background. Genetic association studies recently suggested that the G-allele in patatin-like phospholipase-3 (PNPLA3), which encodes adiponutrin, is strongly associated with hepatic fat content, and more importantly, promotes the susceptibility to develop advanced disease stages with fibrosis $[13,14]$. Thus, the aim of the present study was to extend lipidomic analysis data on the spectrum of NAFLD, and its associated comorbidities as well as its genetic modifications. Therefore, we performed lipidomic profiling in the serum of healthy controls and patients with NAFLD in order to (1) quantify and compare different circulating phospholipid metabolites; (2) to analyze phospholipids in NAFLD-associated metabolic diseases; and finally to (3) study the association between specific circulating lipids and PNPLA3 polymorphism.

\section{Materials and Methods}

\subsection{Study Cohort}

97 patients were recruited from the Department of Internal Medicine at the University Hospital of Heidelberg from 2013-2016. 28 patients served as healthy controls. These control patients had normal liver enzyme levels and did not have any record of liver disease, and therefore showed no indication for liver biopsy. All individuals had routine clinical and hematological examinations. NAFLD was suspected by the presence of elevated liver enzymes and after exclusion of other causes of liver disease (e.g., viral or autoimmune hepatitis, M. Wilson, hemochromatosis, significant alcohol use). In addition, ultrasound examinations and transient elastography with FibroScan ${ }^{\circledR} 502$ Touch (Echosens ${ }^{\mathrm{TM}}$ ) were conducted. Liver biopsy was performed in 41 patients. The diagnosis of NASH was based on the following criteria: (1) Elevated liver enzymes (AST > $46 \mathrm{U} / \mathrm{L}$, ALT $>50 \mathrm{U} / \mathrm{L}$ ); (2) hepatic steatosis on ultrasound; and (3) histological examination or (4) elevated Fibroscan results ( $>7 \mathrm{kPa}) .42$ patients were assigned to the NASH cohort according to these criteria. 25 patients were included in the NAFL group: 5 patients because of liver histology results and 20 patients who exhibited only one or two of the above mentioned criteria. Blood samples of the 97 patients were taken and immediately prepared, and stored at $-80^{\circ} \mathrm{C}$ for further analysis, as shown below. Genotype of the PNPLA3 rs738409 gene of 52 patients was investigated after patient informed consent. The institutional review board approved the study.

\subsection{Profiling of Phospholipids and Sphingolipids}

The patterns of phospholipids and sphingolipids were determined by using XBridge C18 Column from Waters, Milford, MA, USA and HPLC-ESI/MSMS (triple-quadrupole Micro Mass Quattro 81 Premier, Waters, Milford, MA, USA, coupled with HTC Pal auto sampler, CTC Analytics, Zwingen, Switzerland), as described in Jiao et al. [15]. In brief, a constant amount of internal standards, containing 17:0 lysophosphatidylcholine (lysoPC), 14:0/14:0 phosphatidylcholine (PC), 12:0/12:0 phosphatidylethanolamine (PE), and 17:0 Ceramide (Cer), were added to each serum sample. 
A classical Folch method, which uses chloroform/methanol (3:2, v/v), was applied to the glass tubes [16]. After evaporation, lipid extracts were resolved in methanol and were analyzed using HPLC-ESI/MSMS. For the purpose of data acquisition and processing, Masslynx version 4.1 software (Waters, Milford, MA, USA) was used.

\subsection{Data Analysis}

Data are presented as mean \pm SEM and were analyzed with GraphPad Prism 5.0 (GraphPad Software, La Jolla, CA, USA). Groups were compared using unpaired two-tailed students' $t$-test. One-way ANOVA, including Bonferroni post-hoc test, was used for comparing three or more groups. A $p$-value less than 0.05 was considered statistically significant.

\section{Results}

\subsection{Baseline Characteristics}

The study population included 69 patients, who presented at the Department of Internal Medicine at the University Hospital of Heidelberg with the suspected diagnosis of NAFLD. 28 patients served as healthy controls. All patients underwent clinical examination, laboratory data were collected and additionally, ultrasound examinations and transient elastography were performed. Forty-one patients underwent liver biopsy. According to the histological assessment, 5 patients were diagnosed with NAFL and 34 patients were grouped as NASH patients. Two patients were excluded from the study, as they had no pathological findings indicating NAFLD. Based on Fibroscan values (F2-F4), further 8 patients were added to the NASH group. 20 patients were included in the NAFL cohort, as they presented with Fibroscan values under F2. NASH $(n=42)$ and NAFL $(n=25)$ patients had significantly higher levels of liver enzymes, triglycerides and total cholesterol in comparison to healthy controls $(n=28)$ (Table 1).

Table 1. Baseline characteristics and laboratory results from healthy controls, NAFL and NASH patients.

\begin{tabular}{cccc}
\hline & Control $(\boldsymbol{n}=\mathbf{2 8})$ & NAFL $(\boldsymbol{n}=\mathbf{2 5})$ & NASH $(\boldsymbol{n}=\mathbf{4 2})$ \\
\hline Age (years) & $39.4 \pm 2.6$ & $46.4 \pm 2.9$ & $43.7 \pm 2.2$ \\
Sex (M/F) & $13 / 15$ & $14 / 11$ & $29 / 13$ \\
AST (U/L) & $20.7 \pm 1.3$ & $43.6 \pm 5.8^{* * *}$ & $55.3 \pm 6.0^{* * *}$ \\
ALT (U/L) & $22.0 \pm 1.7$ & $68.9 \pm 7.9^{* * *}$ & $86.3 \pm 9.5^{* * *}$ \\
GGT (U/L) & $21.8 \pm 2.4$ & $144.4 \pm 24.6^{* * *}$ & $218.1 \pm 49.6^{* *}$ \\
Bilirubin (mg/dL) & $0.6 \pm 0.04$ & $0.9 \pm 0.2^{*}$ & $1.4 \pm 0.6$ \\
Triglycerides (mg/dL) & $83.4 \pm 5.9$ & $152.2 \pm 12.6^{* * *}$ & $187.3 \pm 14.6^{* * *}$ \\
Total cholesterol (mg/dL) & $182.2 \pm 9.0$ & $213.2 \pm 7.8^{*}$ & $169.3 \pm 12.2$ \\
HDL cholesterol (mg/dL) & $54.7 \pm 3.9$ & $53.1 \pm 3.7$ & $46.7 \pm 2.2$ \\
LDL cholesterol (mg/dL) & $110.8 \pm 6.4$ & $128.2 \pm 6.6$ & $113.3 \pm 6.2$ \\
\hline
\end{tabular}

$\mathrm{M}=$ Male, $\mathrm{F}=$ Female, AST = Aspartate aminotransaminase, ALT = Alanine aminotransaminase, GGT $=$ Gamma-glutamyl transpeptidase, $\mathrm{HDL}=$ High-density lipoproteins, $\mathrm{LDL}=\mathrm{Low}$-density lipoprotein. Values are represented in mean \pm SEM. $p$-values vs. control. ${ }^{*} p$-value $<0.05,{ }^{* *} p$-value $<0.01,{ }^{* * *} p$-value $<0.001$.

\subsection{Increase of Circulating PC and Sphingomyelin and Decrease of Phosphatidylethanolamine in NAFL and $\mathrm{NASH}$}

By using LC-MS/MS, we identified and quantified 140 species of phospholipids and sphingomyelin (SM) in the serum. The total content of PC and SM in the serum was significantly increased in NAFL and NASH patients, compared to healthy controls (Figure 1A,C). Contrary to PC, the level of lysophosphatidylcholine (LPC) showed a trend to be decreased in both the NAFL and NASH groups, without reaching significance (Figure 1D). Circulating phosphatidylethanolamine (PE) was elevated in patients with NASH, compared to those with NAFL (Figure 1B). The amount of lysophosphatidylethanolamine (LPE) was significantly decreased in NAFL and NASH patients in 
comparison to controls (Figure 1E). The level of phosphatidylinositol (PI) and plasmalogens did not differ between all groups (Figure 1F,G).

A

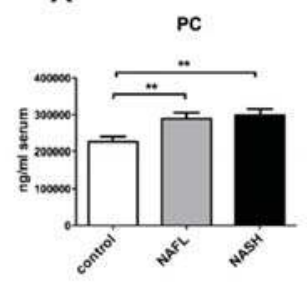

D

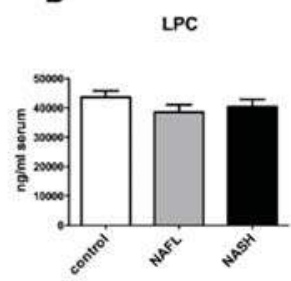

B

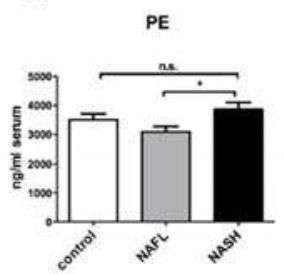

E

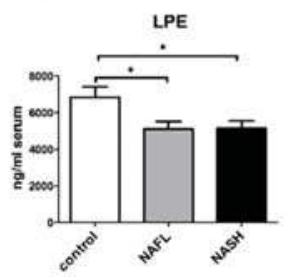

C

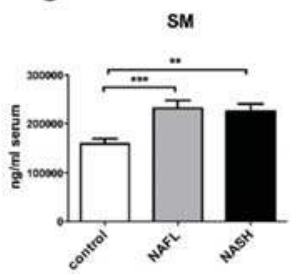

F

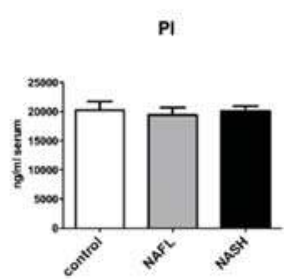

G

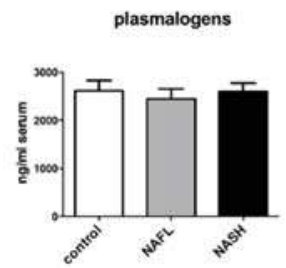

Figure 1. Circulating phospholipids in control $(n=28)$, NAFL $(n=25)$ and NASH $(n=42)$ cohorts. Results of lipid profiling of 95 patients by using LC-MS/MS method. Patients were divided in NAFL and NASH cohorts. 28 patients served as healthy control. Quantification of (A) PC; (B) PE; (C) SM; (D) LPC; (E) LPE; (F) PI; and (G) plasmalogens in the serum of healthy, NAFL and NASH patients in $\mathrm{ng} / \mathrm{mL}$ serum. All the values are presented in mean \pm SEM. ${ }^{*} p<0.05,{ }^{* *} p<0.01,{ }^{* * *} p<0.001$, n.s. = not significant $(p>0.05)$.

\subsection{Altered Phospholipid Composition in NASH Patients with Metabolic Risk Factors}

We investigated the contribution of phospholipid species in NAFLD-associated comorbidities. NAFL and NASH patients were enrolled in groups regarding the presence of diabetes, hypertension and obesity. Interestingly, NAFLD patients with hypertension exhibited significantly higher levels of PC and SM (Figure 2A,B) in the serum than NAFLD patients without hypertension. Circulating PC species, containing linoleic acids (PC 34:2, PC 36:2, PC 38:2) and $\alpha$-linolenic acids (PC 34:3, PC 36:3, PC 38:3), were markedly increased in these patients, whereas those containing arachidonic acid remained unaffected (Figure 2D-G). The sum of phospholipids, including PC, PE and SM, did not differ between diabetes $(n=18)$ and non-diabetes NAFLD patients $(n=49)$ (Figure 3A). However, further analysis revealed a correlation between MUFA-PE and hyperglycemia within the NAFLD cohort. 16 patients with a blood glucose level (BGL) over $100 \mathrm{mg} / \mathrm{dL}$ showed higher levels of serum PE, which was due to a significant elevation of MUFA-PE, whereas PUFA-PE remained unchanged (Figure 3B). Obese NAFLD patients (body mass index (BMI) $>30 \mathrm{~kg} / \mathrm{m}^{2}$ ) showed no significant differences, compared to healthy $\left(B M I<25 \mathrm{~kg} / \mathrm{m}^{2}\right.$ ) and overweight (BMI 25-30 kg/m²) NAFLD patients (Supplementary Materials Figure S1). 


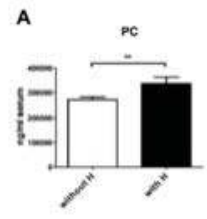

C

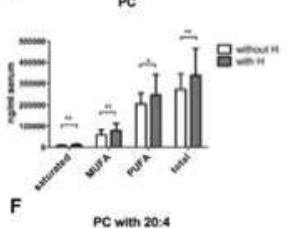

PC with 20:4

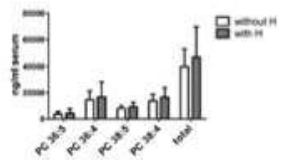

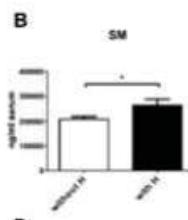

D

Unolek axid

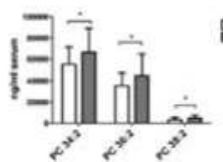

G

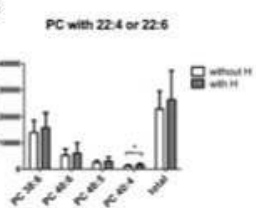

E

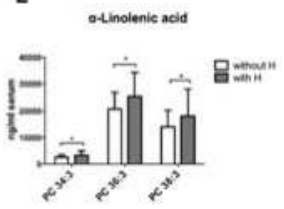

Figure 2. Overview of circulating phospholipids in NAFLD patients with and without hypertension. NAFLD patients were divided into patients with hypertension (with $\mathrm{H} ; n=24$ ) and without hypertension (without $\mathrm{H} ; n=43$ ). (A) Quantification of circulating PC and SM is represented in $\mathrm{ng} / \mathrm{mL}$ serum. (B) Distribution of PC subtypes, namely saturated, mono- and poly-unsaturated PC levels, are displayed in these two groups in $\mathrm{ng} / \mathrm{mL}$ serum. The total content of PC is evaluated as a sum of saturated and unsaturated PC. (C-E) Circulating PC, containing linoleic acids (PC 34:2, PC 36:2, PC 38:2) and $\alpha$-linolenic acids (PC 34:3, PC 36:3, PC 38:3), are compared between hypertensive and normotensive NAFLD patients. (F,G) The amount of arachidonic acid (PC 20:4, 22:4 and 22:6) in PC is demonstrated in this graphic. All the values are presented in mean $\pm \mathrm{SEM}$. ${ }^{*} p<0.05,{ }^{* *} p<0.01$.
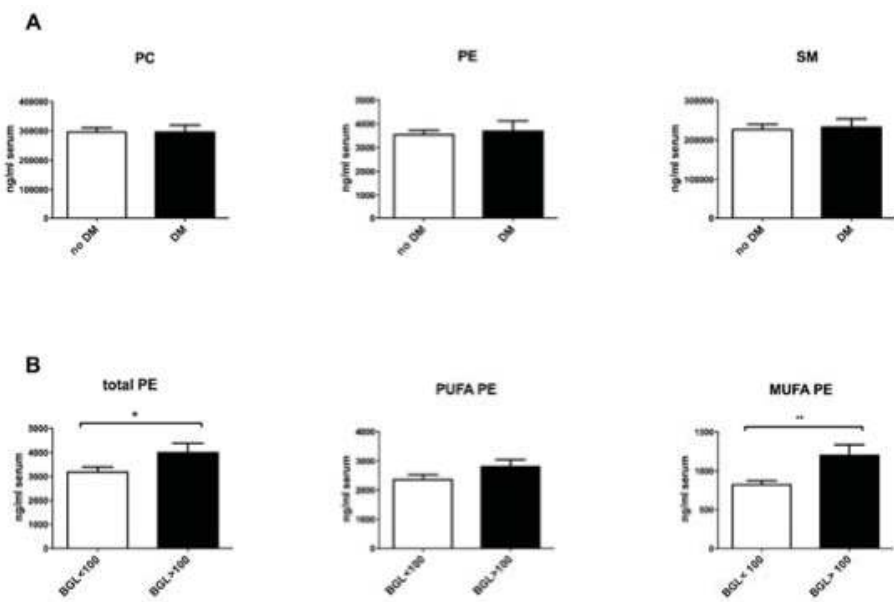

Figure 3. MUFA-PE is increased in NAFLD patients with hyperglycemia. (A) Quantification of circulating PC, PE and SM in ng/mL serum in NAFLD patients who were divided into patients with diabetes mellitus (DM; $n=18$ ) and without diabetes mellitus (no DM; $n=49$ ). (B) Quantification of total PE, PUFA-PE and MUFA-PE in $\mathrm{ng} / \mathrm{mL}$ serum in NAFLD patients with a BGL over $100 \mathrm{mg} / \mathrm{dL}$ $(\mathrm{BGL}>100 ; n=16)$ or under $100 \mathrm{mg} / \mathrm{dL}(\mathrm{BGL}<100 ; n=22)$. All the values are presented in mean \pm SEM. ${ }^{*} p<0.05,{ }^{* *} p<0.01$ 


\subsection{Reduction of Circulating Plasmalogens in Patients with PNPLA3 GG-Genotype}

Genetic analysis regarding the determination of the PNPLA3 rs738409 genotype was performed in 52 NAFLD patients. Lipid profiling of these patients showed lower levels of total plasmalogens, especially 16:0 and 18:1, in patients with PNPLA3 GG-genotype $(n=14)$, compared to those with CC$(n=20)$ or CG- $(n=18)$ allele (Figure 4$)$.

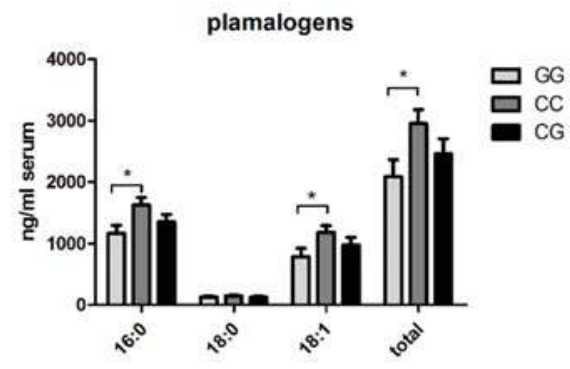

Figure 4. Association between plasmalogens and PNPLA3 gene polymorphism. The PNPLA3 rs738409 genotype was determined in 52 NAFLD patients. The total level of plasmalogen was the sum of plasmalogen 16:0, 18:0 and 18:1. The amount of these plasmalogen subtypes was quantified in NAFLD patients with GG- $(n=14)$, CC- $(n=20)$ and CG-allele $(n=18)$ of PNPLA3. Circulating plasmalogen subclasses are displayed in $\mathrm{ng} / \mathrm{mL}$ serum. All the values are presented in mean $\pm \mathrm{SEM} .{ }^{*} p<0.05$.

\section{Discussion}

It is well known that NAFLD is the hepatic manifestation of the metabolic syndrome [1]. Our extended lipidomic analysis provides an insight into the complex changes in circulating lipid patterns and lipid levels related to NAFLD-associated metabolic risk factors, as well as to the individual genetic background, potentially provoking metabolic and immunomodulatory responses in liver.

It has been recently discovered that altered PC metabolism is involved in the progression of human NAFL to NASH [7]. We also confirm hereby that the content of circulating PC is increased in NAFL and NASH patients, compared to healthy controls. We could not measure a significant change in the PC product, i.e., LPC in our cohorts. One possible explanation is the further utilization of LPC, e.g., by autotaxin. It has been recently reported that plasma autotaxin levels are increased in different chronic liver diseases, including NASH [17]. Total PE content was elevated in NASH patients, compared to NAFL in our study. However, data on PE analysis were inconsistent. Puri et al. and Wattacheril et al. showed that PE levels seemed to be unchanged in NASH compared to healthy liver $[7,9]$, but as recently presented by Ma et al., accumulation of PE could be associated with disease progression [18].

However, we discovered that LPE might serve as a novel biomarker for NAFL and NASH, as this phospholipid subgroup was significantly reduced in the NAFLD cohort. We suggest that the lack of LPE and consequently the inability to further metabolize LPE to PC, can lead to disease progression. To compensate the decrease of hepatocellular PC, therapeutic approaches, including PC supplementation by food intake, were conducted [19]. Stremmel et al. showed a protective and anti-inflammatory role of topical PC in chronic ulcerative colitis [20]. However, the treatment of liver diseases with PC was not as successful in humans as in mouse models, because a replenishment of high PC levels in hepatocytes was not easily achievable [21,22]. As direct application of PC is of no therapeutic benefit, pro-drugs, serving as PC precursors, have been developed $[23,24]$. For instance, our group synthetized a drug candidate, which consists of the bile acid (UDCA) and the PC precursor LPE [25], and which owns hepatoprotective and anti-inflammatory functions in a high fat diet mouse model of NAFLD by changing hepatic fatty acid composition $[24,26]$. 
Our LC-MS/MS analyses revealed that circulating PC, containing linoleic (LA) and $\alpha$-linolenic acids (ALA), was increased in NAFLD patients with hypertension. With changing nutritional behavior over the last few decades, western diet is now characterized by high fat diet and suboptimal micronutrient intake [3]. In particular, intake and metabolism of essential fatty acids are relevant to the pathogenesis of different chronic diseases [27,28]. Essential PUFAs, which are not produced by the human body, include LA and ALA $[19,29]$. Few epidemiological studies suggest a positive impact of dietary intake of essential PUFAs on cholesterol profiling or blood pressure [19,30]. However, these associations seem to be inconsistent in the literature, as other investigations imply the opposite effect or do not recognize any difference [27,31]. Additionally, an excess of LA can lead to adverse effects because of an increase of oxidized LA metabolites [29]. For instance, oxidized LDL compounds derive from LA and contribute to atherosclerosis [32]. In vitro studies demonstrate pro-inflammatory properties of LA, during liver injury by stimulating endoplasmatic reticulum stress and hepatocyte apoptosis and by increasing pro-inflammatory cytokines, such as TNF $\alpha$, which leads to Kupffer cell activation [33,34]. Accordingly, in clinical settings, oxidized derivates of LA, such as 9- and 13-HODE, are correlated with NASH [11]. LA and ALA can also be converted to arachidonic acids [29], which remained unaltered in our cohorts. Our data indicate a positive association between circulating essential fatty acids and NAFLD-associated hypertension.

Although we did not discover lipid alterations by comparing diabetes and non-diabetes NAFLD patients, further analysis regarding BGLs revealed an increase of PE, especially MUFA-PE, in NAFLD patients with a BGL over $100 \mathrm{mg} / \mathrm{dL}$. Glycation of PE could lead to an Amadori-linked product, which could induce lipid peroxidation. The level of Amadori-PE was increased in diabetic patients and was considered as a possible marker of a hyperglycemic condition, particularly in the early stage of diabetes $[35,36]$.

In our next step, our lipid profiling in the NAFLD group identified a decrease of plasmalogens in patients with the GG-genotype of PNPLA3, compared to those with the CC- and CG-genotypes.

Recent studies highlighted a strong association between a variant (rs738409 C > G p. I148M) in the PNPLA3 gene and steatosis, fibrosis and the development of hepatocellular carcinoma in multiple chronic liver diseases, including metabolic disorders, alcoholic liver disease and chronic viral hepatitis. The I148M substitution represents a loss of function mutant that contributes to triglyceride accumulation in hepatocytes, which may lead to increased lipid peroxidation and oxidative stress (lipotoxicity) [37,38]. A recent study by Carpino et al. analyzed serum from NAFLD patients with I148M variant, showing high levels of serum systemic oxidative stress markers, such as F2-isoprostanes and Nox2 activity [37].

Plasmalogens are synthesized in peroxisomes and prevent cellular damage induced by oxidative stress, and thus, seem to have anti-inflammatory functions [39,40]. As recently published by Jang and colleagues, endogenous hepatic plasmalogens appear to be involved in fatty acid metabolism and inhibit steatosis and NASH progression through PPAR $\alpha$-dependent signaling [41]. It is known that oxidative stress-induced peroxisome dysfunction limits plasmalogen biosynthesis, and thus, attenuates the release of plasmalogens to the blood [39]. Puri et al. reported low circulating serum plasmalogen levels in patients presenting with NASH in their lipidomics study [10].

Interestingly, we discovered a positive association of PNPLA3 I148M carriers and low levels of circulating plasmalogens, compared to patients with CC- or CG-genotype. This observation suggests a possible pathogenetic link on the cellular level, underlining the association between triglyceride accumulation, oxidative stress, peroxisome dysfunction and accordingly, defects of plasmologens biosynthesis. Moreover, their loss of function may aggravate NASH progression. This molecular mechanism between PNPLA3 I148M mutant and plasmologens still remains unclear and needs to be elucidated in further experimental and clinical studies.

In recent publications, the most frequently used categorization of NAFLD patients in NASH and NAFL was due to histological assessment. In our cohort study, 28 patients denied liver biopsy in spite of medical recommendation. Ultrasound examination and transient elastography with Fibroscan were 
performed in all sessions. Therefore, 39 patients could be divided in NAFL and NASH subgroups based on histological analyses. Eight patients exhibited liver stiffness $>$ F2 by transient elastography and were included to the NASH group. This kind of categorization may lead to sample selection bias. However, lipid profiling in 39 patients with only histological assessment revealed a significant increase of high levels of PC and SM and a marked decrease of LPE in the NASH cohort (34 patients), compared to healthy controls, consistent with the lipid analysis in our groups with results of histology and transient elastography. No significant deviation could be detected between NAFL and control patients. It should be noted that only 5 individuals from 39 patients with histological analyses could be assigned to the NAFL group (Supplementary Materials Figure S2).

\section{Conclusions}

In summary, this lipidomic profiling investigated circulating phospholipid metabolites in NAFLD patients. This current study confirmed that PC is significantly increased in the serum of NASH patients and is identified as a possible novel biomarker in the pathogenesis of NAFLD, namely LPE. Our analysis elucidated lipidomic alterations in NAFLD patients with hypertension, demonstrating a key role for PC species containing essential fatty acids in NAFLD-associated hypertension. Furthermore, metabolic profiling in this NAFLD cohort determined a correlation between high levels of MUFA-PE and hyperglycemia. Finally, we discovered a link between low levels of circulating plasmalogens and NAFLD patients with the unfavorable GG genotype of PNPLA3.

Supplementary Materials: The following are available online at http:/ /www.mdpi.com/2072-6643/10/5/649/s1. Figure S1: Comparison of phospholipid subclasses in NAFLD-associated obesity. Figure S2: Quantification of PL species in healthy controls $(n=28)$, NAFL $(n=5)$ and NASH $(n=34)$ cohorts.

Author Contributions: S.T.-H. and A.P. conceived and designed the experiments; S.T.-H. and H.G.-S. performed the experiments; S.T.-H. and W.C. analyzed the data; A.P. contributed reagents/materials/analysis tools; S.T.-H. and A.P. wrote the paper.

Funding: A.P. was funded by the German Research Foundation (PA 2365/1-1) and by the Olympia Morata Postdoctoral Fellowship of the Medical Faculty of University of Heidelberg.

Conflicts of Interest: The authors declare no conflict of interest.

\section{References}

1. Williams, C.D.; Stengel, J.; Asike, M.I.; Torres, D.M.; Shaw, J.; Contreras, M.; Landt, C.L.; Harrison, S.A. Prevalence of Nonalcoholic Fatty Liver Disease and Nonalcoholic Steatohepatitis among a Largely Middle-Aged Population Utilizing Ultrasound and Liver Biopsy: A Prospective Study. Gastroenterology 2011, 140, 124-131. [CrossRef] [PubMed]

2. Bellentani, S.; Scaglioni, F.; Marino, M.; Bedogni, G. Epidemiology of non-alcoholic fatty liver disease. Dig. Dis. 2010, 28, 155-161. [CrossRef] [PubMed]

3. Myles, I.A. Fast food fever: Reviewing the impacts of the Western diet on immunity. Nutr. J. 2014, 13, 61. [CrossRef] [PubMed]

4. Contos, M.J.; Sanyal, A.J. The clinicopathologic spectrum and management of nonalcoholic fatty liver disease. Adv. Anat. Pathol. 2002, 9, 37-51. [CrossRef] [PubMed]

5. Bedossa, P.; Poitou, C.; Veyrie, N.; Bouillot, J.L.; Basdevant, A.; Paradis, V.; Tordjman, J.; Clement, K. Histopathological algorithm and scoring system for evaluation of liver lesions in morbidly obese patients. Hepatology 2012, 56, 1751-1759. [CrossRef] [PubMed]

6. Malhi, H.; Gores, G. Molecular Mechanisms of Lipotoxicity in Nonalcoholic Fatty Liver Disease. Semin. Liver Dis. 2008, 28, 360-369. [CrossRef] [PubMed]

7. Puri, P.; Baillie, R.A.; Wiest, M.M.; Mirshahi, F.; Choudhury, J.; Cheung, O.; Sargeant, C.; Contos, M.J.; Sanyal, A.J. A lipidomic analysis of nonalcoholic fatty liver disease. Hepatology 2007, 46, 1081-1090. [CrossRef] [PubMed]

8. Anjani, K.; Lhomme, M.; Sokolovska, N.; Poitou, C.; Aron-Wisnewsky, J.; Bouillot, J.-L.; Lesnik, P.; Bedossa, P.; Kontush, A.; Clement, K.; et al. Circulating phospholipid profiling identifies portal contribution to NASH signature in obesity. J. Hepatol. 2015, 62, 905-912. [CrossRef] [PubMed] 
9. Wattacheril, J.; Seeley, E.H.; Angel, P.; Chen, H.; Bowen, B.P.; Lanciault, C.; Caprioli, R.M.; Abumrad, N.; Flynn, C.R. Differential Intrahepatic Phospholipid Zonation in Simple Steatosis and Nonalcoholic Steatohepatitis. PLoS ONE 2013, 8, e57165. [CrossRef] [PubMed]

10. Puri, P.; Wiest, M.M.; Cheung, O.; Mirshahi, F.; Sargeant, C.; Min, H.-K.; Contos, M.J.; Sterling, R.K.; Fuchs, M.; Zhou, H.; et al. The plasma lipidomic signature of nonalcoholic steatohepatitis. Hepatology 2009, 50, 1827-1838. [CrossRef] [PubMed]

11. Feldstein, A.E.; Lopez, R.; Tamimi, T.A.-R.; Yerian, L.; Chung, Y.-M.; Berk, M.; Zhang, R.; McIntyre, T.M.; Hazen, S.L. Mass spectrometric profiling of oxidized lipid products in human nonalcoholic fatty liver disease and nonalcoholic steatohepatitis. J. Lipid Res. 2010, 51, 3046-3054. [CrossRef] [PubMed]

12. Loomba, R.; Quehenberger, O.; Armando, A.; Dennis, E.A. Polyunsaturated fatty acid metabolites as novel lipidomic biomarkers for noninvasive diagnosis of nonalcoholic steatohepatitis. J. Lipid Res. 2015, 56, 185-192. [CrossRef] [PubMed]

13. Romeo, S.; Kozlitina, J.; Xing, C.; Pertsemlidis, A.; Cox, D.; Pennacchio, L.A.; Boerwinkle, E.; Cohen, J.C.; Hobbs, H.H. Genetic variation in PNPLA3 confers susceptibility to nonalcoholic fatty liver disease. Nat. Genet. 2008, 40, 1461-1465. [CrossRef] [PubMed]

14. Krawczyk, M.; Grünhage, F.; Zimmer, V.; Lammert, F. Variant adiponutrin (PNPLA3) represents a common fibrosis risk gene: Non-invasive elastography-based study in chronic liver disease. J. Hepatol. 2011, 55, 299-306. [CrossRef] [PubMed]

15. Jiao, L.; Gan-Schreier, H.; Zhu, X.; Wei, W.; Tuma-Kellner, S.; Liebisch, G.; Stremmel, W.; Chamulitrat, W. Ageing sensitized by iPLA2 $\beta$ deficiency induces liver fibrosis and intestinal atrophy involving suppression of homeostatic genes and alteration of intestinal lipids and bile acids. Biochim. Biophys. Acta Mol. Cell Biol. Lipids 2017, 1862, 1520-1533. [CrossRef] [PubMed]

16. Folch, J.; Lees, M.; Sloane Stanley, G.H. A simple method for the isolation and purification of total lipides from animal tissues. J. Biol. Chem. 1957, 226, 497-509. [PubMed]

17. Kaffe, E.; Katsifa, A.; Xylourgidis, N.; Ninou, I.; Zannikou, M.; Harokopos, V.; Foka, P.; Dimitriadis, A.; Evangelou, K.; Moulas, A.N.; et al. Hepatocyte autotaxin expression promotes liver fibrosis and cancer. Hepatology 2017, 65, 1369-1383. [CrossRef] [PubMed]

18. Ma, D.W.L.; Arendt, B.M.; Hillyer, L.M.; Fung, S.K.; McGilvray, I.; Guindi, M.; Allard, J.P. Plasma phospholipids and fatty acid composition differ between liver biopsy-proven nonalcoholic fatty liver disease and healthy subjects. Nutr. Diabetes 2016, 6, e220. [CrossRef] [PubMed]

19. Küllenberg, D.; Taylor, L.A.; Schneider, M.; Massing, U. Health effects of dietary phospholipids. Lipids Health Dis. 2012, 11, 3. [CrossRef] [PubMed]

20. Stremmel, W.; Merle, U.; Zahn, A.; Autschbach, F.; Hinz, U.; Ehehalt, R. Retarded release phosphatidylcholine benefits patients with chronic active ulcerative colitis. Gut 2005, 54, 966-971. [CrossRef] [PubMed]

21. Lieber, C.S. Alcoholic fatty liver: Its pathogenesis and mechanism of progression to inflammation and fibrosis. Alcohol 2004, 34, 9-19. [CrossRef] [PubMed]

22. Lieber, C.S.; Weiss, D.G.; Groszmann, R.; Paronetto, F.; Schenker, S.; Veterans Affairs Cooperative Study 391 Group II. Veterans Affairs Cooperative Study of Polyenylphosphatidylcholine in Alcoholic Liver Disease. Alcohol. Clin. Exp. Res. 2003, 27, 1765-1772. [CrossRef] [PubMed]

23. Abdelmalek, M.F.; Angulo, P.; Jorgensen, R.A.; Sylvestre, P.B.; Lindor, K.D. Betaine, a promising new agent for patients with nonalcoholic steatohepatitis: Results of a pilot study. Am. J. Gastroenterol. 2001, 96, 2711-2717. [CrossRef] [PubMed]

24. Chamulitrat, W.; Liebisch, G.; Xu, W.; Gan-Schreier, H.; Pathil, A.; Schmitz, G.; Stremmel, W. Ursodeoxycholyl Lysophosphatidylethanolamide Inhibits Lipoapoptosis by Shifting Fatty Acid Pools toward Monosaturated and Polyunsaturated Fatty Acids in Mouse Hepatocytes. Mol. Pharmacol. 2013, 84, 696-709. [CrossRef] [PubMed]

25. Chamulitrat, W.; Burhenne, J.; Rehlen, T.; Pathil, A.; Stremmel, W. Bile salt-phospholipid conjugate ursodeoxycholyl lysophosphatidylethanolamide as a hepatoprotective agent. Hepatology 2009, 50, 143-154. [CrossRef] [PubMed]

26. Pathil, A.; Mueller, J.; Warth, A.; Chamulitrat, W.; Stremmel, W. Ursodeoxycholyl lysophosphatidylethanolamide improves steatosis and inflammation in murine models of nonalcoholic fatty liver disease. Hepatology 2012, 55, 1369-1378. [CrossRef] [PubMed] 
27. Bemelmans, W.J.E.; Lefrandt, J.D.; Feskens, E.J.M.; van Haelst, P.L.; Broer, J.; Meyboom-de Jong, B.; May, J.F.; Tervaert, J.W.C.; Smit, A.J. Increased $\alpha$-linolenic acid intake lowers C-reactive protein, but has no effect on markers of atherosclerosis. Eur. J. Clin. Nutr. 2004, 58, 1083-1089. [CrossRef] [PubMed]

28. Berry, E. Are diets high in omega-6 polyunsaturated fatty acids unhealthy? Eur. Heart J. Suppl. 2001, 3, D37-D41. [CrossRef]

29. Santoro, N.; Caprio, S.; Feldstein, A.E. Oxidized metabolites of linoleic acid as biomarkers of liver injury in nonalcoholic steatohepatitis. Clin. Lipidol. 2013, 8, 411-418. [CrossRef] [PubMed]

30. Stamler, J.; Rose, G.; Stamler, R.; Elliott, P.; Dyer, A.; Marmot, M.; Brown, I.J.; Tzoulaki, I.; Saitoh, S.; Dyer, A.R.; et al. INTERSALT study findings. Public health and medical care implications. Hypertension 1989, 14, 570-577. [CrossRef] [PubMed]

31. Scherhag, R.; Kramer, H.J.; Düsing, R. Dietary administration of eicosapentaenoic and linolenic acid increases arterial blood pressure and suppresses vascular prostacyclin synthesis in the rat. Prostaglandins 1982, 23, 369-382. [CrossRef]

32. Spiteller, D.; Spiteller, G. Oxidation of Linoleic Acid in Low-Density Lipoprotein: An Important Event in Atherogenesis. Angew. Chem. Int. Ed. 2000, 39, 585-589. [CrossRef]

33. Böhm, T.; Berger, H.; Nejabat, M.; Riegler, T.; Kellner, F.; Kuttke, M.; Sagmeister, S.; Bazanella, M.; Stolze, K.; Daryabeigi, A.; et al. Food-derived peroxidized fatty acids may trigger hepatic inflammation: A novel hypothesis to explain steatohepatitis. J. Hepatol. 2013, 59, 563-570. [CrossRef] [PubMed]

34. Zhang, Y.; Xue, R.; Zhang, Z.; Yang, X.; Shi, H. Palmitic and linoleic acids induce ER stress and apoptosis in hepatoma cells. Lipids Health Dis. 2012, 11, 1. [CrossRef] [PubMed]

35. Sookwong, P.; Nakagawa, K.; Fujita, I.; Shoji, N.; Miyazawa, T. Amadori-Glycated Phosphatidylethanolamine, a Potential Marker for Hyperglycemia, in Streptozotocin-Induced Diabetic Rats. Lipids 2011, 46, 943-952. [CrossRef] [PubMed]

36. Miyazawa, T.; Oak, J.-H.; Nakagawa, K. Tandem Mass Spectrometry Analysis of Amadori-Glycated Phosphatidylethanolamine in Human Plasma. Ann. N. Y. Acad. Sci. 2005, 1043, 280-283. [CrossRef] [PubMed]

37. Carpino, G.; Pastori, D.; Baratta, F.; Overi, D.; Labbadia, G.; Polimeni, L.; Di Costanzo, A.; Pannitteri, G.; Carnevale, R.; Del Ben, M.; et al. PNPLA3 variant and portal/periportal histological pattern in patients with biopsy-proven non-alcoholic fatty liver disease: A possible role for oxidative stress. Sci. Rep. 2017, 7, 15756. [CrossRef] [PubMed]

38. Trépo, E.; Romeo, S.; Zucman-Rossi, J.; Nahon, P. PNPLA3 gene in liver diseases. J. Hepatol. 2016, 65, 399-412. [CrossRef] [PubMed]

39. Wallner, S.; Schmitz, G. Plasmalogens the neglected regulatory and scavenging lipid species. Chem. Phys. Lipids 2011, 164, 573-589. [CrossRef] [PubMed]

40. Brites, P.; Waterham, H.R.; Wanders, R.J. Functions and biosynthesis of plasmalogens in health and disease. Biochim. Biophys. Acta Mol. Cell Biol. Lipids 2004, 1636, 219-231. [CrossRef] [PubMed]

41. Jang, J.E.; Park, H.-S.; Yoo, H.J.; Baek, I.-J.; Yoon, J.E.; Ko, M.S.; Kim, A.-R.; Kim, H.S.; Park, H.-S.; Lee, S.E.; et al. Protective role of endogenous plasmalogens against hepatic steatosis and steatohepatitis in mice. Hepatology 2017, 66, 416-431. [CrossRef] [PubMed]

(C) 2018 by the authors. Licensee MDPI, Basel, Switzerland. This article is an open access article distributed under the terms and conditions of the Creative Commons Attribution (CC BY) license (http:/ / creativecommons.org/licenses/by/4.0/). 


\title{
Review \\ Beneficial and Paradoxical Roles of Anti-Oxidative Nutritional Support for Non-Alcoholic Fatty Liver Disease
}

\author{
Daisuke Uchida ${ }^{\circledR}$, Akinobu Takaki *, Takuya Adachi and Hiroyuki Okada \\ Department of Gastroenterology and Hepatology, Okayama University Graduate School of Medicine, \\ Dentistry and Pharmaceutical Sciences, 2-5-1 Shikata-cho, Kita-ku, Okayama 700-8558, Japan; \\ d.uchida0309@gmail.com (D.U.); adataku719@yahoo.co.jp (T.A.); hiro@md.okayama-u.ac.jp (H.O.) \\ * Correspondence: akitaka@md.okayama-u.ac.jp; Tel.: +81-86-235-7219
}

Received: 1 July 2018; Accepted: 24 July 2018; Published: 27 July 2018

\begin{abstract}
Oxidative stress is being recognized as a key factor in the progression of chronic liver disease (CLD), especially non-alcoholic fatty liver disease (NAFLD). Many NAFLD treatment guidelines recommend the use of antioxidants, especially vitamin E. Many prospective studies have described the beneficial effects of such agents for the clinical course of NAFLD. However, as these studies are usually short-term evaluations, lasting only a few years, whether or not antioxidants continue to exert favorable long-term effects, including in cases of concomitant hepatocellular carcinoma, remains unclear. Antioxidants are generally believed to be beneficial for human health and are often commercially available as health-food products. Patients with lifestyle-related diseases often use such products to try to be healthier without practicing lifestyle intervention. However, under some experimental NAFLD conditions, antioxidants have been shown to encourage the progression of hepatocellular carcinoma, as oxidative stress is toxic for cancer cells, just as for normal cells. In this review, we will highlight the paradoxical effects of antioxidants against NAFLD and related hepatocellular carcinoma.
\end{abstract}

Keywords: non-alcoholic fatty liver disease; hepatocellular carcinoma; anti-oxidant

\section{Introduction}

Non-alcoholic fatty liver disease (NAFLD) is a common chronic liver disease associated with obesity and metabolic syndrome [1,2]. The deposition of lipids in the liver induces inflammation, insulin resistance, and hepatic steatosis [3]. Non-alcoholic steatohepatitis (NASH) is a severe form of NAFLD that causes cirrhosis and hepatocellular carcinoma (HCC) because of persistent inflammation associated with hepatic steatosis $[4,5]$. The mechanism underlying the development of NASH from simple steatosis, namely non-alcoholic fatty liver (NAFL), has been considered as either a two-hit theory or a multiple hit-process [6-8]. The two-hit theory comprises a first hit of hepatic steatosis and second hit of several cellular stress responses, such as oxidative stress or endoplasmic reticulum (ER) stress. Given that the second hits are multifactorial and hepatic steatosis may be induced via hepatic inflammation, the process has recently been expressed as a multiple parallel hit process or multiple hit process [8].

Oxidative stress is a cellular stress associated with NASH progression. Reactive oxygen species (ROS) are generated during free fatty acid metabolism in microsomes, peroxisomes, and mitochondria, and comprise an established source of oxidative stress [9]. Oxidative stress is increased through the generation of ROS, as well as by defects in redox defense mechanisms involving glutathione (GSH), catalase or superoxide dismutase (SOD), and it induces various events related to not only NASH progression but also carcinogenesis such as DNA damage, tissue remodeling, and alterations 
in gene expression [10,11]. Mitochondria play a key role in the development of oxidative stress, and the dysfunction of mitochondria may induce NASH progression. Some reports have shown that antioxidant therapies, such as vitamin E, improve NASH, but the mechanism, especially concerning malignant tumor progression, and long-term outcomes are not clear [12,13].

In the present study, we reviewed the relationship between antioxidant nutrients and NASH progression including the carcinogenic risk.

\section{The Relationship between NAFLD and Oxidative Stress}

Oxidative stress is strongly related to chronic hepatic inflammation, and NAFLD/NASH is no exception, being included in the "second hit" of NASH progression as well as apoptosis, ER stress, and intestinal environment. It is widely known that oxidative stress functions as an important regulator of the progression of liver steatosis [14-16]. Various cellular stresses, including oxidative stress, apoptosis, and gut-derived signals such as lipopolysaccharide (LPS), trigger an inflammatory response and progressive liver damage [17]. Chronic oxidative stress correlates with a variety of pathologies such as malignant diseases, diabetes mellitus, cardiovascular diseases, chronic inflammatory diseases, and aging acceleration. Oxidative stress is caused by the generation of ROS, which have various causes, including interactions among gut microbiota. When excess ROS are produced excessively or the endogenous antioxidant capacity is diminished, indiscriminate oxidation elicits harmful effects resulting in oxidative stress [18].

Insulin resistance and dyslipidemia are widely accepted as disease progression factors in NAFLD [7]. Insulin signals suppress gluconeogenesis and enhance lipid synthesis in the liver. A human hepatic mRNA analysis revealed that the expression of insulin receptor substrate (IRS)-2, which mediates the effects of insulin by acting as a molecular adopter, was decreased while that of gluconeogenesis enzymes was increased in both NAFL and NASH [19]. Insulin resistance is clinically evident in the advanced stage of NAFLD; however, hepatic insulin resistance at the molecular level started from the NAFL stage. Altered cholesterol homeostasis such as increased cholesterol synthesis and uptake or reduced cholesterol excretion have been shown in NAFLD [20]. The hepatic free cholesterol accumulation has been shown to damage mitochondria and ER function thereby inducing hepatic inflammation and fibrosis [21]. Lipoprotein lipase (LPL), a key regulator of fatty acid release from triglyceride-rich lipoproteins, controls the cellular uptake of fatty acid and triglyceride accumulation [22]. The inhibition of LPL may prevent the accumulation of hepatic lipid [23].

Changes in the alimentary tract environment, which is connected to the liver via the portal vein, including the oral microbiota to the intestinal and colorectal microbiota have been shown to be strongly related to various diseases. The gut microbiota, which comprise various species such as Bifidobacterium, Bacteroides, Clostridium, Lactobacillales, and Prevotella, produce endogenous ethanol that induces the formation of ROS by hepatic stellate cells and Kupffer cells [24]. The composition of the microbiota depends on the age, health history, food intake, and probiotics consumed [25]. The food intake strongly correlates with dyslipidemia and is a crucial factor to consider when deciding on treatments for NAFLD, as it influences the gut microbiota [26].

An imbalance in the microbiome can affect the intestine and liver via microbiota-related signaling pathways such as toll-like receptor (TLR) signaling activation. LPL can also be suppressed by a fastinginduced adipose factor, which is regulated by the gut microbiota via TLR ligands [7,27]. A TLR-4 ligand LPS is liberated from the outer membrane of Gram-negative bacteria and binds to lipopolysaccharide binding protein (LBP). These ligands stimulate TLR signals, including nuclear factor kappa (NF-kB) with cluster of differentiation (CD) 14, and induce the production of inflammatory cytokines [28]. LPS is increased by a high-fat, high-calorie diet, as well as dyslipidemia and insulin resistance, which are related to NASH progression. The involvement of TLR-2 in NASH inflammation and fibrosis has been investigated in mouse NASH models [29]. A serum multiple cytokine analysis revealed that the levels of the chemokine interferon gamma inducible protein 10 (Interferon gamma-induced protein (IP)-10; C-X-C motif chemokine (CXCL)-10 increased stepwise from healthy volunteers to NAFL and NASH 
patients. A TLR-2 ligand peptidoglycan in combination with an in vitro insulin resistance condition (i.e., high glucose with insulin in the culture medium) increased the expression of IP-10 mRNA, indicating the importance of insulin resistance with bacterial signals in NASH progression [30]. Therefore, the diet helps to establish a close relationship among the gut microbiota, oxidative stress and NAFLD/NASH.

\section{Treatments for NAFLD/NASH}

The treatment strategy of NAFLD involves intervention with diet, exercise, and drugs [13,31-34]. Lifestyle interventions, including diet and exercise, are strongly related to insulin resistance and dyslipidemia. A randomized, controlled trial testing simple weight loss gain by lifestyle intervention using a combination of diet, exercise, and behavior modification resulted in NASH pathological improvement [35]. Insulin resistance has been shown to be an independent predictor of NASH in biopsy-proven cases that were likely to be controllable by any method, including lifestyle intervention [36]. Dyslipidemia has various causes, such as hereditary predisposition, a sedentary lifestyle, and a high calorie intake. The improvement of dyslipidemia may resolve NASH progression, and nutritional support, such as low-fat and low-calorie diets, holds a prominent position in treatment approaches [37,38].

Vitamin E is the most commonly used antioxidant treatment for NASH [39]. Pioglitazone, an antihyperglycemic drug, is reported to ameliorate liver injury and fibrosis in patients with NASH with and without diabetes [33]. Sanyal et al. reported that vitamin E therapy was effective for NAFLD improvement compared with pioglitazone and placebo and recommended antioxidant treatment as well as lifestyle intervention, bariatric surgery, dyslipidemia treatment, and diabetes treatment [13]. On the basis of these data, the American Association for the Study of Liver Diseases (AASLD) recommends treating NASH with vitamin E [40]. However, the Treatment of NAFLD in Children (TONIC) randomized trial showed that neither metformin nor vitamin $\mathrm{E}$ improved the liver function in patients with NAFLD [41]. A meta-analysis of randomized controlled trials revealed that vitamin E and pioglitazone but not metformin improved the histological activity of NASH [42]. However, the efficacy of these drugs is still controversial, and further clinical studies including assessments of long-term outcomes should be conducted.

Probiotics are reported to be a promising treatment option for NAFLD, improving the liver function, obesity, and insulin resistance [43]. Shavakhi et al. reported that metformin in combination with probiotics improved liver inflammation to a significantly greater degree than metformin alone [44]. This effect may be caused by the association between the gut microbiota and liver, which receives blood from the intestine through the portal vein.

Surgical treatments, such as bariatric surgery and liver transplantation, are conceivable treatments for NAFLD/NASH. Bariatric surgery for obesity is effective for improving NASH, as weight loss can improve dyslipidemia and hepatic fibrosis $[45,46]$. It also decreases the mortality rate due to diabetes, cardiology events, and malignant tumors. Liver transplantation is another treatment option for NAFLD/NASH related end-stage cirrhosis [47]. However, NASH can occur after liver transplantations, and continuous medical approaches are needed [48].

\section{Carcinogenesis and Cancer Progression in Correlation with Oxidative Stress and Antioxidant Treatment}

Hepatocellular carcinoma (HCC) is the major malignant liver tumor and the main cause of death in NAFLD/NASH patients. Oxidative stress is strongly associated with hepatocarcinogenesis and cancer progression. However, the effects on cancer-free patients and patients with HCC are likely different. Oxidative stress has a driving effect on carcinogenesis while also exerting toxicity against cancer cells. This contradiction will be discussed in this section. 


\subsection{Oxidative Stress in NASH-Hepatocarcinogenesis}

Oxidative stress is reported to induce various events related to carcinogenesis such as DNA damage, tissue remodeling, and alterations in gene expression $[11,49,50]$. ROS production is stimulated by inflammatory factors, and oxidative stress promotes oncogenic transformation due to DNA-damage. ROS activates various pathways, such as Wingless-type MMTV intefration site family (Wnt) / $\beta$-catenin, NF-kB, or myc proto-oncogene protein (c-Myc)/Transforming growth factor (TGF)- $\alpha$. This activation also occurs in carcinogenesis in liver cancer. Inducible nitric oxide synthase (iNOS), which produces nitrogen monoxides (NO), correlates with chronic inflammation, cell proliferation, DNA repair, migration, and angiogenesis [51]. The iNOS activity is important for endothelial stress gene expression, which protects against oxidative stress, and may be the key modulator of hepatocarcinogenesis.

8-hydroxydeoxyguanosine (8-OHdG), which is produced by DNA-damage with ROS, reflects the amount of oxidative stress and generates a point mutation in DNA double strands. Kawanishi et al. reported that $8-\mathrm{OHdG}$, which is a reliable marker for oxidative stress in the liver, strongly correlates with infection-related carcinogenesis via chronic inflammation [52]. Seki et al. reported that the 8-OHdG expression in the liver tissue is related to the pathological features of NAFLD and hepatocarcinogenesis [53].

Adenosine monophosphate activated protein kinase (AMPK) is a highly conserved heterodimeric serine-threonine kinase that plays a role in cellular energy homeostasis and different fundamental cellular processes such as the cellular proliferation, survival, and metabolism. It serves as an energy sensor in eukaryotic cells and bridges metabolism to carcinogenesis [54]. Its activated form (phospho (p)-AMPK) is down-regulated in HCC tissues from patients and low levels of p-AMPK correlate with a poor prognosis, indicating the importance of AMPK signaling in HCC [55]. AMPK signaling has recently been indicated to correlate with inflammatory responses, as the energy metabolism in immune cells is related to immunoregulation [56]. Under many oxidative stress-related conditions, such as diabetes or dyslipidemia, a diminished AMPK activity has been shown to be associated with tissue oxidative stress $[57,58]$.

As mentioned above, oxidative stress is linked to the progression from NAFLD to HCC. The correlation of AMPK and clinical conditions encourage us to control AMPK as a cancer regulator.

\subsection{Antioxidant Treatment Effects on Prevention of NASH-Hepatocarcinogenesis}

Given that advanced chronic liver disease is the strongest risk factor for hepatocarcinogenesis, antioxidant treatments can be an important approach to preventing HCC development from NASH. The effect of these agents concerning whether they have the properties to protect against hepatocarcinogenesis should include an improvement in liver fibrosis improvement.

Vitamin E was reported to prevent hepatocarcinogenesis via the down-regulation of iNOS and nicotinamide adenine dinucleotide phosphate (NADPH) oxidase [59]. Many clinical studies have reported that vitamin E improves NAFLD and prevents the development of HCC [13,60-62]. However, these studies were all performed over a relatively short period (i.e., within two years). Hepatocarcinogenesis progresses from NAFL/NASH to cirrhosis over a long period of time, and middle-aged men carry risks of hepatocarcinogenesis even at an early stage of NAFLD [63]. The long-term outcomes must be evaluated in order to judge the true efficacy of antioxidant therapy for NASH-hepatocarcinogenesis.

Many candidate antioxidant agents may show favorable effects on NASH and NASHrelated hepatocarcinogenesis.

Anti-diabetic agents such as metformin, pioglitazone, glucagon-like peptide 1-receptor agonists (GLP-1 RAs), and dipeptidyl-peptidase-4 inhibitors (DPP-4Is) were recently found to show favorable effects on the NAFLD clinical course $[7,64,65]$. Metformin and pioglitazone in particular are regarded as anti-oxidant agents. Metformin is the most well-studied AMPK-activating agent. Metformin-related AMPK pathway activation is involved in many cell types including T cells, B cells, hepatocytes, and even liver fibrosis-inducing hepatic stellate cells (HSCs). In in vitro and in vivo models (mice), metformin suppressed alpha smooth muscle actin ( $\alpha$-SMA) expression via AMPK activation and the 
inhibition of the succinate-related pathway in (HSCs) [66]. HSC activation is an important step in hepatocarcinogenesis. In addition, metformin induces the production of the antioxidant enzyme, heme oxygenase-1 (HO-1) in human endothelial cells via the nuclear factor erythroid 2-related factor (Nrf2) signaling pathway [67]. Determining metformin's long-term effects on reducing the risk of hepatocarcinogenesis might require further long-term studies.

Pioglitazone is a thiazolidinedione activating peroxisome proliferator-activated receptor (PPAR) $\gamma$ that improves the insulin resistance and is used to treat type 2 diabetes. A six-month randomized study of pioglitazone revealed a reduction in necroinflammation of liver histopathology with no reduction in fibrosis [68]. L-carnitine is an essential nutrient and an important molecule in regulating mitochondrial and peroxisomal metabolism [69]. A recent randomized controlled study indicated histological improvement of liver fibrosis after 18 months with pioglitazone for NASH in patients with concomitant type 2 diabetes [70]. This agent may therefore be effective for inhibiting NAFL progression to NASH and hepatocarcinogenesis.

Flavonoids are heterogeneous polyphenols found in various plants, such as fruits, vegetables, and green tea that reportedly exert antioxidative function protecting the liver tissue from damage caused by ROS [71]. A mixture of flavonolignans and minor polyphenolic compounds, derived from the milk thistle plant (Silybum marianum) known as silymarin, is said to be an antioxidative agent [72]. Salomone et al. reported that silibinin, the main component of silymarin, restored nicotinamide adenine dinucleotide (NAD+) levels, and played a protective role against NAFLD [73]. In a randomized, double-blind, placebo-controlled study, silymarin at $700 \mathrm{mg}$ three times daily for 48 weeks significantly ameliorated liver fibrosis in NASH patients [74]. Silymarin may therefore be a promising agent for the treatment of NAFLD/NASH patients.

L-carnitine acts as an antioxidant mediator by controlling the $\beta$-oxidation cycle and adenosine triphosphate (ATP) generation [75]. L-carnitine is well known as a fat-burning supplement; however, it has recently attracted attention as an anti-cancer agent because of its effects as an antioxidant, apoptosis inducer, and inhibitor of histone deacetylase [76-79]. We previously reported that L-carnitine controls the mitochondrial function in hepatic non-cancerous tissue from a mouse NASH model by upregulating the Lactobacillales population related to the secondary bile acid production in the gut microbiota [76]. L-carnitine does not eliminate oxidative stress but rather controls the oxidative balance to aid the mitochondrial function. This might be a better approach to regulating oxidative stress.

High levels of plasma free fatty acids increase the levels of hepatic free fatty acids. Long-chain fatty acids taken up by mitochondria as complexes with $\mathrm{L}$-carnitine are subsequently metabolized in $\beta$-oxidation pathways. Under oxidative stress, oxidative reactions convert oxidized cofactors (NAD+ and flavin adenine dinucleotide (FAD)) into reduced cofactors (nicotinamide adenine dinucleotide (NADH) and flavin adenine dinucleotide H2 (FADH2)) and deliver electrons to the respiratory chain. An imbalance between the increased delivery of electrons to the respiratory chain and the decreased outflow from the respiratory chain causes electrons and ROS products to accumulate. Antioxidant defenses, such as superoxide dismutase (SOD), glutathione peroxidase (GPx), or catalase, can metabolize $\mathrm{O}_{2}$. and $\mathrm{H}_{2} \mathrm{O}_{2}$ to non-toxic $\mathrm{H}_{2} \mathrm{O}$. However, the Fenton and/or Haber-Weiss reactions generate highly reactive, toxic, hydroxyl radicals $(\mathrm{OH})$. Vitamin $\mathrm{E}$ as a general cytotoxic ROS scavenger erases oxidative stress. Metformin activates AMPK and induces antioxidant gene transcription, and 5-aminoimidazole-4-carboxamide-1- $\beta$-D-ribofuranoside (AICAR) activates Nrf2 via a mechanism possibly similar to that of metformin. L-carnitine supports the mitochondrial function to increase the long-chain fatty acid uptake. In NASH patients, oxidative stress works as toxic from NASH progression to cancer development. However, in cancer patients, oxidative stress should be maintained to some degree in order to control cancer via toxic oxidative stress reactions. To control cancer progression, AMPK/mammalian target of rapamycin (mTOR) signaling has an important function that would be a target to intervene. 


\subsection{Antioxidant Treatment Effects on Liver Cancer}

Various treatments have been proposed for HCC, such as surgical approaches (e.g., liver resection, liver transplantation), radio frequency ablation, transcatheter arterial chemoembolization, and chemotherapy [80]. However, HCC remains incurable because of the low response rate and frequent recurrence based on chronic liver inflammation and fibrosis. Many reports have described the usage of antioxidant therapies for HCC, but their efficacy still remains unclear [81-83].

Metformin inhibits the cell growth of HCC by regulating AMPK activation and inducing apoptosis [84]. The effect of metformin on NASH-related HCC in mouse models has been reported, but the clinical effect in humans is unclear [85].

The AMPK activator 5-aminoimidazole-4-carboxamide-1- $\beta$-D-ribofuranoside (AICAR) activates Nrf2 protein, which binds to Kelch-like ECH associated protein 1 (Keap1), a protein that exists in the cytoplasm in an inactive form [86]. AICAR may be a candidate for treating liver cancer.

Flavonoid and polyphenols have been shown to exert antitumor effects through the control of various molecular pathways [81,87]. Curcumin is an antioxidant polyphenol compound reported to exert antitumor activities by inhibiting the vascular endothelial growth factor (VEGF) expression and phosphoinositide 3-kinase (PI3K)/v-Akt murine thymoma viral oncogene (Akt) signaling [82]. The newly confirmed antioxidant silymarin has also been analyzed for its utility in preventing hepaticarcinogenesis [73].

L-carnitine is a mitochondria supporting agent that may be useful for controlling oxidative stress in HCC patients. Our previous report showed that the tumor number and maximum tumor size were decreased in NASH-HCC mouse models than control diet [76]. In that experiment, L-carnitine was administered from the early onset of NASH, and the liver tumor outcomes were assessed afterwards. Under such conditions, L-carnitine improved the NASH pathogenesis resulting in decreased NASH related hepatocarcinogenesis and so is difficult to conclude whether its use is beneficial for HCC. To answer this point, we next examined the effect of L-carnitine administration after hepatocarcinogenesis in NASH-related cholangiocarcinoma-like tumors [88]. L-carnitine resulted in a good outcome for this model, suggesting it might be an effective antioxidant even after cancer development.

As above, many candidate antioxidant treatments have been proposed for HCC; however, the clinical investigations on this point are inadequate at present. To administer antioxidants for cancer stages, we must consider the oxidative stress-related environment adequately (Figure 1).

Recently, oral chemotherapeutic multi-kinase inhibitors such as sorafenib have been widely used to control advanced-stage HCC. Sorafenib inhibits tumor cell proliferation and angiogenesis through the inactivation of vascular endothelial growth factor receptor (VEGFR), platelet-derived growth factor receptor (PDGFR), and the serine-threonine kinase Raf-1, which participates in the Rat sarcoma (Ras)/rapidly accelerated fibrosarcoma (Raf)/mitogen-activated protein kinase kinase (MEK)/mitogen-activated protein kinase (MAPK) signal cascade [89]. Recently, other multi-kinase inhibitors such as lenvatinib, regorafenib, cabozantinib, and ramucirumab have also been developed [90-92]. Recent clinical experience with sorafenib has indicated that acquired drug resistance can occur over a long period of drug administration. Thus, in these stages of HCC, the effects of accompanying agents such as antioxidants on the drug resistance should be investigated. Accumulating data indicate that autophagy and phosphoinositide 3-kinase (PI3K)/Akt signaling are associated with acquired resistance to sorafenib in HCC. The AMPK/mTOR signaling pathway had been demonstrated to be important in autophagy. A high sustained glucose condition produces advanced glycation products (AGEs) which damage many organs including the liver. The receptor for AGE (RAGE) has been shown to be overexpressed in HCC promoting proliferation. In addition, RAGE induced sorafenib resistance in in vitro and in vivo xenograft models [93]. A reduction in RAGE was also found to be able to increase autophagy and eliminate sorafenib resistance in vitro via the AMPK pathway activation. Metformin was able to reduce RAGE expression and helped rescue cells from sorafenib resistance. Metformin might therefore be useful for eliminating sorafenib resistance in 
HCC; however, one clinical study reported a bad outcome for patients administered metformin with sorafenib [94].

Oxidative stress in NASH: carcinogenesis initiator

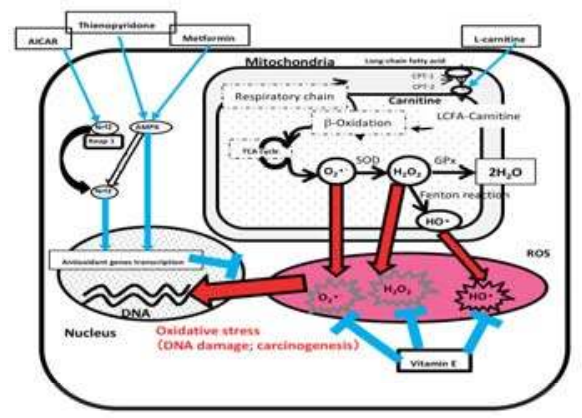

Oxidative stress in liver cancer: carcinogenesis initiator and anti-cancer response

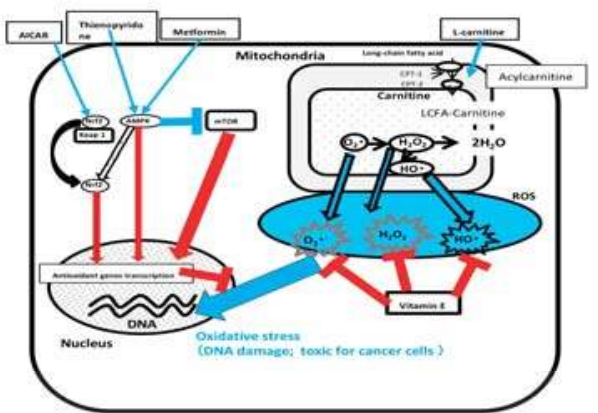

Figure 1. Oxidative stress and anti-oxidant in non-alcoholic steatohepatitis (NASH) and liver cancer. Red lines; unfavorable effects, Blue lines; favorable effects, AICAR, 5-aminoimidazole-4carboxamide-1- $\beta$-D-ribofuranoside; Nrf2, nuclear factor erythroid 2-related factor; AMPK, AMP-activated protein kinase; Keap1, Kelch-like enoyl-CoA hydratase $(\mathrm{ECH})$ associated protein; Nrf2, nuclear factor erythroid 2-related factor; CPT, carnitine palmitoyltransferase; SOD, superoxide dismutase; GPx, glutathione peroxidase; LCFA-carnitine, long-chain acylcarnitine; TCA-cycle, tricarboxylic acid cycle.

Administering sorafenib efficacy-supporting agents is an approach to resolving drug resistance as mentioned above with metformin. The oxidative stress-inducing agent tetrandrine, a bisbenzylisoquinoline alkaloid, has been shown to exert synergistic anti-tumor activity with sorafenib by increasing ROS [95]. More data on such combination therapy with sorafenib will be required to draw more solid conclusions.

Immune checkpoint molecules including programmed cell death-1 (PD-1) and programmed cell death-1 ligand (PD-L1), are promising new therapeutic agents for the treatment of various cancers including HCC [96]. Nivolumab, an anti-PD-1 antibody, was approved by the Food and Drug Administration (FDA) and is a key drug for the treatment of HCC dramatically improving the survival of many cancers, including solid-tumors; however, 30 to 50\% of patients are unresponsive [97]. To overcome this unresponsiveness, several combination treatments have been attempted. An in vivo analysis of CD8+ T cells treated with PD-1 antibody revealed that a highly proliferative fraction could be found in the draining lymph node indicating that tumor priming was necessary for CD8+ T cell activation [98]. These highly proliferative CD8+ T cells contained more cellular ROS, a larger mitochondrial mass, higher mitochondrial potential, and more mitochondrial superoxide than CD8+ T cells without PD-1 antibody treatment, indicating the activation of mitochondria in T cells. Several mitochondrial function activators have been tested for their additive effects of PD-1 blockade with successful results. Of them, the PPAR- $\alpha$ agonist bezafibrate showed a particularly promising response. Given that bezafibrate is a standard agent for treating high triglyceride levels, this combination might be useful for combination therapy. The relationship between oxidative stress, antioxidants, and PD-1/PD-L1 needs further study and clinical trials to resolve the issue of a lack of responsiveness to anti-PD-1 antibody therapy.

\subsection{Cautions of Antioxidants on Liver Cancer Initiation and Maintenance}

Whether or not antioxidant agents prevent cancer risks remains controversial. The U.S. Preventive Services Task Force (USPSTF) stated that the current evidence is insufficient to assess the balance of benefits and harms of vitamins [99]. In particular, excessive doses of vitamins may induce negative 
effects. Four randomized controlled trials (RCTs) of vitamin E included in the USPSTF comments showed no significant effect on the incidence of all types of cancer or cancer mortality rates. However, one RCT assessing the risk of prostate cancer with vitamin E administration found a 17\% increase in the prostate cancer incidence with this supplement [100]. Since a large prospective study to confirm the HCC risks for NASH is difficult to perform and requires a long observation time (although there are some ongoing studies), we should attempt to determine the optimum treatment approach based on short-term data from small populations and experimental data.

Oxidative stress is a crucial event for cancer initiation; however, it also has important roles in cancer prevention. Stem cell-like cancer cells have powerful antioxidative properties that protect them from oxidative stress and thus prevent self-apoptosis [101]. The expression of CD44 variant 9 (CD44v9) has recently been investigated as a functional marker of stem-like cells in many types of cancers [102]. The interaction of CD44v9 with xc- Substrate-specific Subunit (xCT), also called the glutamate/cysteine antiporter solute carrier family 7 member 11 (SLC7A11), a subunit of the glutamate-cystine transporter system xc-, stabilizes the latter and thereby potentiates cancer cells to promote glutathione synthesis and reduce the activity of cellular redox system, resulting in resistance to toxic oxidative stresses [103]. Inducing oxidative stress in cancer patients is an approach that is being investigated as a cancer treatment in several clinical trials [104]. However, this approach is likely to also be toxic to normal cells and may lead to the induction of further carcinogenesis. Furthermore, given that treatment-resistant cancer stem cells can still escape toxic oxidative stress, maintaining moderate oxidative stress is a critical point of anti-oxidative stress treatment for cancer patients.

Mitochondrial function-supporting agents, such as metformin or L-carnitine, might be good therapies to investigate in large-scale prospective studies in humans. In cancer patients, the drug effects should be analyzed according to the stages of the cancer. When tumors have been completely resected or ablated, a standard anti-oxidant approach may be effective for preventing new hepatocarcinogenesis. When tumors have not been ablated completely but can be managed with local treatment, such as transcatheter arterial chemo-embolization (TACE), mitochondrial function-supporting agents, such as metformin or L-carnitine, might be useful approaches for controlling the oxidative-antioxidative stress balance. When tumors are not being treated with local therapies but instead managed with anti-cancer agents, presently multi-kinase inhibitors such as sorafenib, regorafenib and lenvatinib, oxidative stress may be an important cellular stress to cancer cells not to be eliminated. As mentioned in previous section, oxidative stress-inducing agents may compound the effects of sorafenib [95]. However, even the favorably reported antioxidant metformin might adversely affect the clinical outcome in patients with HCC receiving the multi-kinase inhibitor sorafenib [94].

L-carnitine is a candidate drug that has shown good efficacy even after cancer has already developed. We previously investigated the effects of the standard anti-oxidant agents vitamin $\mathrm{E}$ and L-carnitine and found that only L-carnitine reduced the number of liver tumors [88]. Vitamin E administration induced HO-1 protein expression in cancer tissue, resulting in an increase in the number of stem-like cancer cells. Furthermore, L-carnitine administration improved the Lactobacillales population and the balance of bile acid (primary to secondary bile acid), while vitamin E did not affect the intestinal environment. These findings show that vitamin E strongly ameliorates the oxidative stress in both cancer cells and normal cells, while L-carnitine works as an "oxidative stress balancer". An "oxidative stress eraser" such as vitamin E might therefore be unsuitable for the treatment of cancer patients. However, even L-carnitine carries the possibility of an increased risk of experimental HCC [105]. A mouse HCC model of diethylnitrosamine (DEN)-injected mice fed a high-fat diet showed the predominance of acylcarnitine species in the tumors according to a metabolomic profiling analysis. Intracellular long-chain fatty acids are esterified to acyl-coenzyme A and conjugated with carnitine resulting in acylcarnitine by carnitine palmitoyltransferase 1 (CPT 1). The acylcarnitine is then converted back to acyl-coenzyme A by CPT2 and enters the $\beta$-oxidation pathway. Of note, the CPT2 expression was diminished in the HCC tumors inducing acylcarnitine deposition. In this mouse model, L-carnitine supplementation resulted in an increased amount of acylcarnitines and enhanced HCC 
tumorigenesis. These conflicting results suggest that the HCC-preventive effects of L-carnitine might require a preserved CPT2 function in order to avoid inducing the accumulation of acylcarnitines.

It should be noted that almost all antioxidants can elicit unfavorable effects on carcinogenesis under certain conditions. We must bear these facts in mind and thus perform antioxidant supplementation judiciously. We can use antioxidant for NASH, while should decide whether to stop after HCC development (Figure 2).

A proposal for anti-oxidant treatment application in NAFLD

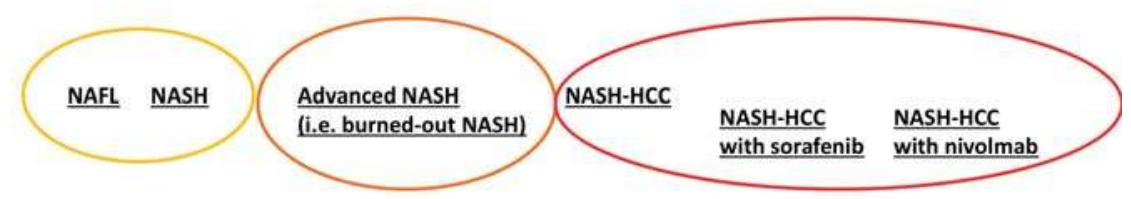

Clinical

Evidence

(guideline

recommend)

\begin{tabular}{|l|l|l|}
\hline $\begin{array}{l}\text { Proposal from } \\
\text { experimental } \\
\text { data }\end{array}$ & $\begin{array}{l}\text { Vitamin E } \\
\text { Pioglitazone }\end{array}$ \\
& $\begin{array}{l}\text { Metformin } \\
\text { Silymarin }\end{array}$ \\
& GLP-1 RA \\
& DPP-4 Is \\
& L-carnitine \\
\hline
\end{tabular}

Vitamin $\mathrm{E}$
No data

No data

No data

Metformi

Silymarin

Metformin

Silymarin

Pioglitazone

L-carnitine
Tetrandrine

(oxidative stress-inducer)
No data

Figure 2. A proposal for the antioxidant treatment application in non-alcoholic fatty liver disease (NAFLD). NAFL, non-alcoholic fatty liver; NASH, Non-alcoholic steatohepatitis; HCC, hepatocellular carcinoma; GLP-1 RA, glucagon-like peptide 1-receptor agonist; DPP-4 Is, dipeptidyl-peptidase-4 inhibitors.

Several guidelines recommend vitamin E be administered for NASH, as some prospective studies proved to be beneficial for laboratory data and histological activity. However, there are no prospective data on NASH with diminished activity and steatosis with advanced fibrosis, such as burned-out NASH. There are also no data on NASH-HCC. Many experimental studies recommend antioxidant agents be administered for patients with NAFL and NASH. Given that advanced NASH often shows no steatosis, some antioxidants may be more suitable for these patients than others. For NASH-HCC, given that oxidative stress is an important stress response for regulating cancer cells, strong antioxidants may actually be harmful. When we administer oxidative stress-related agents together with sorafenib and other multi-kinase inhibitors, combination effects may be exerted by oxidative stress-inducing agents. The AMPK activator metformin has been shown to be beneficial for HCC, although clinical evidence is still being discussed. Nivolmab is a next-step HCC treatment approach for activating the cancer immune response. Bezafibrate has been shown to exert additive effects to activate the $\mathrm{T}$ cell response.

\section{Conclusions}

NAFLD is a major lifestyle-related disease, and nutritional support is an important approach to its treatment. Antioxidant therapy, such as vitamin E administration, is effective for preventing NASH progression; however, the long-term outcomes of this therapy are unclear, especially in 
precancerous and cancer patients. Antioxidant therapy should be prescribed according to the clinical condition of the patients, such as those with NAFL, NASH, advanced NASH, cancer with successful ablation, cancer with relative ablation, and advanced cancer requiring anti-cancer agents. In HCC patients, antioxidant treatment should be carefully managed, as these agents can also act against tumor resolution.

Author Contributions: Conceptualization, A.T. and D.U.; Methodology, A.T. and D.U.; Writing-Original Draft Preparation, D.U.; Writing-Review \& Editing, A.T., T.A.; Supervision, H.O.

Funding: This research received no external funding.

Conflicts of Interest: The authors declare no conflict of interest.

\section{References}

1. Pacifico, L.; Anania, C.; Martino, F.; Poggiogalle, E.; Chiarelli, F.; Arca, M.; Chiesa, C. Management of metabolic syndrome in children and adolescents. Nutr. Metab. Cardiovasc. Dis. 2011, 21, 455-466. [CrossRef] [PubMed]

2. Doycheva, I.; Watt, K.D.; Alkhouri, N. Nonalcoholic fatty liver disease in adolescents and young adults: The next frontier in the epidemic. Hepatology 2017, 65, 2100-2109. [CrossRef] [PubMed]

3. Smith, B.W.; Adams, L.A. Non-alcoholic fatty liver disease. Crit. Rev. Clin. Lab. Sci. 2011, 48, 97-113. [CrossRef] [PubMed]

4. Matteoni, C.A.; Younossi, Z.M.; Gramlich, T.; Boparai, N.; Liu, Y.C.; McCullough, A.J. Nonalcoholic fatty liver disease: A spectrum of clinical and pathological severity. Gastroenterology 1999, 116, 1413-1419. [CrossRef]

5. Ono, M.; Saibara, T. Clinical features of nonalcoholic steatohepatitis in Japan: Evidence from the literature. J. Gastroenterol. 2006, 41, 725-732. [CrossRef] [PubMed]

6. Gentile, C.L.; Pagliassotti, M.J. The role of fatty acids in the development and progression of nonalcoholic fatty liver disease. J. Nutr. Biochem. 2008, 19, 567-576. [CrossRef] [PubMed]

7. Takaki, A.; Kawai, D.; Yamamoto, K. Molecular mechanisms and new treatment strategies for non-alcoholic steatohepatitis (NASH). Int. J. Mol. Sci. 2014, 15, 7352-7379. [CrossRef] [PubMed]

8. Tilg, H.; Moschen, A.R. Evolution of inflammation in nonalcoholic fatty liver disease: The multiple parallel hits hypothesis. Hepatology 2010, 52, 1836-1846. [CrossRef] [PubMed]

9. Pessayre, D. Role of mitochondria in non-alcoholic fatty liver disease. J. Gastroenterol. Hepatol. 2007, 22 (Suppl. S1), 20-27. [CrossRef] [PubMed]

10. Muriel, P. Role of free radicals in liver diseases. Hepatol. Int. 2009, 3, 526-536. [CrossRef] [PubMed]

11. Yongvanit, P.; Pinlaor, S.; Bartsch, H. Oxidative and nitrative DNA damage: Key events in opisthorchiasis-induced carcinogenesis. Parasitol. Int. 2012, 61, 130-135. [CrossRef] [PubMed]

12. Al-Busafi, S.A.; Bhat, M.; Wong, P.; Ghali, P.; Deschenes, M. Antioxidant therapy in nonalcoholic steatohepatitis. Hepat. Res. Treat. 2012, 2012, 947575. [CrossRef] [PubMed]

13. Sanyal, A.J.; Chalasani, N.; Kowdley, K.V.; McCullough, A.; Diehl, A.M.; Bass, N.M.; NeuschwanderTetri, B.A.; Lavine, J.E.; Tonascia, J.; Unalp, A.; et al. Pioglitazone, vitamin E, or placebo for nonalcoholic steatohepatitis. N. Engl. J. Med. 2010, 362, 1675-1685. [CrossRef] [PubMed]

14. Albano, E.; Mottaran, E.; Occhino, G.; Reale, E.; Vidali, M. Review article: Role of oxidative stress in the progression of non-alcoholic steatosis. Aliment. Pharmacol. Ther. 2005, 22 (Suppl. S2), 71-73. [CrossRef] [PubMed]

15. Seki, S.; Kitada, T.; Yamada, T.; Sakaguchi, H.; Nakatani, K.; Wakasa, K. In situ detection of lipid peroxidation and oxidative DNA damage in non-alcoholic fatty liver diseases. J. Hepatol. 2002, 37, 56-62. [CrossRef]

16. Begriche, K.; Igoudjil, A.; Pessayre, D.; Fromenty, B. Mitochondrial dysfunction in NASH: Causes, consequences and possible means to prevent it. Mitochondrion 2006, 6, 1-28. [CrossRef] [PubMed]

17. Csak, T.; Ganz, M.; Pespisa, J.; Kodys, K.; Dolganiuc, A.; Szabo, G. Fatty acid and endotoxin activate inflammasomes in mouse hepatocytes that release danger signals to stimulate immune cells. Hepatology 2011, 54, 133-144. [CrossRef] [PubMed]

18. Ohta, S. Molecular hydrogen as a preventive and therapeutic medical gas: Initiation, development and potential of hydrogen medicine. Pharmacol. Ther. 2014, 144, 1-11. [CrossRef] [PubMed] 
19. Honma, M.; Sawada, S.; Ueno, Y.; Murakami, K.; Yamada, T.; Gao, J.; Kodama, S.; Izumi, T.; Takahashi, K.; Tsukita, S.; et al. Selective insulin resistance with differential expressions of IRS-1 and IRS-2 in human NAFLD livers. Int. J. Obes. 2018. [CrossRef] [PubMed]

20. Musso, G.; Gambino, R.; Cassader, M. Cholesterol metabolism and the pathogenesis of non-alcoholic steatohepatitis. Prog. Lipid Res. 2013, 52, 175-191. [CrossRef] [PubMed]

21. Van Rooyen, D.M.; Larter, C.Z.; Haigh, W.G.; Yeh, M.M.; Ioannou, G.; Kuver, R.; Lee, S.P.; Teoh, N.C.; Farrell, G.C. Hepatic free cholesterol accumulates in obese, diabetic mice and causes nonalcoholic steatohepatitis. Gastroenterology 2011, 141, 1393-1403. [CrossRef] [PubMed]

22. Preiss-Landl, K.; Zimmermann, R.; Hammerle, G.; Zechner, R. Lipoprotein lipase: The regulation of tissue specific expression and its role in lipid and energy metabolism. Curr. Opin. Lipidol. 2002, 13, 471-481. [CrossRef] [PubMed]

23. Zhang, L.; Zhang, Z.; Li, Y.; Liao, S.; Wu, X.; Chang, Q.; Liang, B. Cholesterol induces lipoprotein lipase expression in a tree shrew (Tupaia belangeri chinensis) model of non-alcoholic fatty liver disease. Sci. Rep. 2015, 5, 15970. [CrossRef] [PubMed]

24. Su, G.L. Lipopolysaccharides in liver injury: Molecular mechanisms of Kupffer cell activation. Am. J. Physiol.-Gastrointest. Liver Physiol. 2002, 283, G256-G265. [CrossRef] [PubMed]

25. Gratz, S.W.; Mykkanen, H.; El-Nezami, H.S. Probiotics and gut health: A special focus on liver diseases. World J. Gastroenterol. 2010, 16, 403-410. [CrossRef] [PubMed]

26. Backhed, F.; Ding, H.; Wang, T.; Hooper, L.V.; Koh, G.Y.; Nagy, A.; Semenkovich, C.F.; Gordon, J.I. The gut microbiota as an environmental factor that regulates fat storage. Proc. Natl. Acad. Sci. USA 2004, 101, 15718-15723. [CrossRef] [PubMed]

27. Zak-Golab, A.; Olszanecka-Glinianowicz, M.; Kocelak, P.; Chudek, J. The role of gut microbiota in the pathogenesis of obesity. Postepy Hig. Med. Dosw. (Online) 2014, 68, 84-90. [CrossRef] [PubMed]

28. Miller, Y.I.; Choi, S.H.; Wiesner, P.; Bae, Y.S. The SYK side of TLR4: Signalling mechanisms in response to LPS and minimally oxidized LDL. Br. J. Pharmacol. 2012, 167, 990-999. [CrossRef] [PubMed]

29. Miura, K.; Yang, L.; van Rooijen, N.; Brenner, D.A.; Ohnishi, H.; Seki, E. Toll-like receptor 2 and palmitic acid cooperatively contribute to the development of nonalcoholic steatohepatitis through inflammasome activation in mice. Hepatology 2013, 57, 577-589. [CrossRef] [PubMed]

30. Wada, N.; Takaki, A.; Ikeda, F.; Yasunaka, T.; Onji, M.; Nouso, K.; Nakatsuka, A.; Wada, J.; Koike, K.; Miyahara, K.; et al. Serum-inducible protein (IP)-10 is a disease progression-related marker for non-alcoholic fatty liver disease. Hepatol. Int. 2017, 11, 115-124. [CrossRef] [PubMed]

31. Ekstedt, M.; Franzen, L.E.; Mathiesen, U.L.; Thorelius, L.; Holmqvist, M.; Bodemar, G.; Kechagias, S. Long-term follow-up of patients with NAFLD and elevated liver enzymes. Hepatology 2006, 44, 865-873. [CrossRef] [PubMed]

32. Bradford, V.; Dillon, J.; Miller, M. Lifestyle interventions for the treatment of non-alcoholic fatty liver disease. Hepat. Med. 2014, 6, 1-10. [PubMed]

33. Aithal, G.P.; Thomas, J.A.; Kaye, P.V.; Lawson, A.; Ryder, S.D.; Spendlove, I.; Austin, A.S.; Freeman, J.G.; Morgan, L.; Webber, J. Randomized, placebo-controlled trial of pioglitazone in nondiabetic subjects with nonalcoholic steatohepatitis. Gastroenterology 2008, 135, 1176-1184. [CrossRef] [PubMed]

34. Nelson, J.E.; Roth, C.L.; Wilson, L.A.; Yates, K.P.; Aouizerat, B.; Morgan-Stevenson, V.; Whalen, E.; Hoofnagle, A.; Mason, M.; Gersuk, V.; et al. Vitamin D Deficiency Is Associated with Increased Risk of Non-alcoholic Steatohepatitis in Adults with Non-alcoholic Fatty Liver Disease: Possible Role for MAPK and NF-kappaB? Am. J. Gastroenterol. 2016, 111, 852-863. [CrossRef] [PubMed]

35. Promrat, K.; Kleiner, D.E.; Niemeier, H.M.; Jackvony, E.; Kearns, M.; Wands, J.R.; Fava, J.L.; Wing, R.R. Randomized controlled trial testing the effects of weight loss on nonalcoholic steatohepatitis. Hepatology 2010, 51, 121-129. [CrossRef] [PubMed]

36. Ballestri, S.; Nascimbeni, F.; Romagnoli, D.; Lonardo, A. The independent predictors of non-alcoholic steatohepatitis and its individual histological features. Insulin resistance, serum uric acid, metabolic syndrome, alanine aminotransferase and serum total cholesterol are a clue to pathogenesis and candidate targets for treatment. Hepatol. Res. 2016, 46, 1074-1087. [PubMed]

37. Targher, G.; Bertolini, L.; Rodella, S.; Tessari, R.; Zenari, L.; Lippi, G.; Arcaro, G. Nonalcoholic fatty liver disease is independently associated with an increased incidence of cardiovascular events in type 2 diabetic patients. Diabetes Care 2007, 30, 2119-2121. [CrossRef] [PubMed] 
38. Corey, K.E.; Vuppalanchi, R.; Wilson, L.A.; Cummings, O.W.; Chalasani, N.; Nash, C.R.N. NASH resolution is associated with improvements in HDL and triglyceride levels but not improvement in LDL or non-HDL-C levels. Aliment. Pharmacol. Ther. 2015, 41, 301-309. [CrossRef] [PubMed]

39. Watanabe, S.; Hashimoto, E.; Ikejima, K.; Uto, H.; Ono, M.; Sumida, Y.; Seike, M.; Takei, Y.; Takehara, T.; Tokushige, K.; et al. Evidence-based clinical practice guidelines for nonalcoholic fatty liver disease/nonalcoholic steatohepatitis. J. Gastroenterol. 2015, 50, 364-377. [CrossRef] [PubMed]

40. Chalasani, N.; Younossi, Z.; Lavine, J.E.; Diehl, A.M.; Brunt, E.M.; Cusi, K.; Charlton, M.; Sanyal, A.J. The diagnosis and management of non-alcoholic fatty liver disease: Practice Guideline by the American Association for the Study of Liver Diseases, American College of Gastroenterology, and the American Gastroenterological Association. Hepatology 2012, 55, 2005-2023. [CrossRef] [PubMed]

41. Lavine, J.E.; Schwimmer, J.B.; Van Natta, M.L.; Molleston, J.P.; Murray, K.F.; Rosenthal, P.; Abrams, S.H.; Scheimann, A.O.; Sanyal, A.J.; Chalasani, N.; et al. Effect of vitamin E or metformin for treatment of nonalcoholic fatty liver disease in children and adolescents: The TONIC randomized controlled trial. JAMA 2011, 305, 1659-1668. [CrossRef] [PubMed]

42. Said, A.; Akhter, A. Meta-Analysis of Randomized Controlled Trials of Pharmacologic Agents in Non-alcoholic Steatohepatitis. Ann. Hepatol. 2017, 16, 538-547. [CrossRef] [PubMed]

43. Lavekar, A.S.; Raje, D.V.; Manohar, T.; Lavekar, A.A. Role of Probiotics in the Treatment of Nonalcoholic Fatty Liver Disease: A Meta-analysis. Euroasian J. Hepatogastroenterol. 2017, 7, 130-137. [CrossRef] [PubMed]

44. Shavakhi, A.; Minakari, M.; Firouzian, H.; Assali, R.; Hekmatdoost, A.; Ferns, G. Effect of a Probiotic and Metformin on Liver Aminotransferases in Non-alcoholic Steatohepatitis: A Double Blind Randomized Clinical Trial. Int. J. Prev. Med. 2013, 4, 531-537. [PubMed]

45. Adams, T.D.; Gress, R.E.; Smith, S.C.; Halverson, R.C.; Simper, S.C.; Rosamond, W.D.; Lamonte, M.J.; Stroup, A.M.; Hunt, S.C. Long-term mortality after gastric bypass surgery. N. Engl. J. Med. 2007, 357, 753-761. [CrossRef] [PubMed]

46. Lassailly, G.; Caiazzo, R.; Buob, D.; Pigeyre, M.; Verkindt, H.; Labreuche, J.; Raverdy, V.; Leteurtre, E.; Dharancy, S.; Louvet, A.; et al. Bariatric Surgery Reduces Features of Nonalcoholic Steatohepatitis in Morbidly Obese Patients. Gastroenterology 2015, 149, 379-388. [CrossRef] [PubMed]

47. Wang, X.; Li, J.; Riaz, D.R.; Shi, G.; Liu, C.; Dai, Y. Outcomes of liver transplantation for nonalcoholic steatohepatitis: A systematic review and meta-analysis. Clin. Gastroenterol. Hepatol. 2014, 12, $394-402$. [CrossRef] [PubMed]

48. Dare, A.J.; Plank, L.D.; Phillips, A.R.; Gane, E.J.; Harrison, B.; Orr, D.; Jiang, Y.; Bartlett, A.S. Additive effect of pretransplant obesity, diabetes, and cardiovascular risk factors on outcomes after liver transplantation. Liver Transpl. 2014, 20, 281-290. [CrossRef] [PubMed]

49. Georgakilas, A.G. Oxidative stress, DNA damage and repair in carcinogenesis: Have we established a connection? Cancer Lett. 2012, 327, 3-4. [CrossRef] [PubMed]

50. Vallee, A.; Lecarpentier, Y. Crosstalk between peroxisome proliferator-activated receptor gamma and the canonical wnt/beta-catenin pathway in chronic inflammation and oxidative stress during carcinogenesis. Front. Immunol. 2018, 9, 745. [CrossRef] [PubMed]

51. Sawa, T.; Ohshima, H. Nitrative DNA damage in inflammation and its possible role in carcinogenesis. Nitric Oxide 2006, 14, 91-100. [CrossRef] [PubMed]

52. Kawanishi, S.; Ohnishi, S.; Ma, N.; Hiraku, Y.; Oikawa, S.; Murata, M. Nitrative and oxidative DNA damage in infection-related carcinogenesis in relation to cancer stem cells. Genes Environ. 2016, 38, 26. [CrossRef] [PubMed]

53. Seki, S.; Kitada, T.; Sakaguchi, H. Clinicopathological significance of oxidative cellular damage in non-alcoholic fatty liver diseases. Hepatol. Res. 2005, 33, 132-134. [CrossRef] [PubMed]

54. Hardie, D.G.; Carling, D.; Carlson, M. The AMP-activated/SNF1 protein kinase subfamily: Metabolic sensors of the eukaryotic cell? Annu. Rev. Biochem. 1998, 67, 821-855. [CrossRef] [PubMed]

55. Zheng, L.; Yang, W.; Wu, F.; Wang, C.; Yu, L.; Tang, L.; Qiu, B.; Li, Y.; Guo, L.; Wu, M.; et al. Prognostic significance of AMPK activation and therapeutic effects of metformin in hepatocellular carcinoma. Clin. Cancer Res. 2013, 19, 5372-5380. [CrossRef] [PubMed]

56. Gubser, P.M.; Bantug, G.R.; Razik, L.; Fischer, M.; Dimeloe, S.; Hoenger, G.; Durovic, B.; Jauch, A.; Hess, C. Rapid effector function of memory CD8+ T cells requires an immediate-early glycolytic switch. Nat. Immunol. 2013, 14, 1064-1072. [CrossRef] [PubMed] 
57. Cheng, X.; Siow, R.C.; Mann, G.E. Impaired redox signaling and antioxidant gene expression in endothelial cells in diabetes: A role for mitochondria and the nuclear factor-E2-related factor 2-Kelch-like ECH-associated protein 1 defense pathway. Antioxid. Redox Signal. 2011, 14, 469-487. [CrossRef] [PubMed]

58. Yamauchi, T.; Kamon, J.; Minokoshi, Y.; Ito, Y.; Waki, H.; Uchida, S.; Yamashita, S.; Noda, M.; Kita, S.; Ueki, K.; et al. Adiponectin stimulates glucose utilization and fatty-acid oxidation by activating AMP-activated protein kinase. Nat. Med. 2002, 8, 1288-1295. [CrossRef] [PubMed]

59. Calvisi, D.F.; Ladu, S.; Hironaka, K.; Factor, V.M.; Thorgeirsson, S.S. Vitamin E down-modulates iNOS and NADPH oxidase in c-Myc/TGF-alpha transgenic mouse model of liver cancer. J. Hepatol. 2004, 41, 815-822. [CrossRef] [PubMed]

60. Foster, T.; Budoff, M.J.; Saab, S.; Ahmadi, N.; Gordon, C.; Guerci, A.D. Atorvastatin and antioxidants for the treatment of nonalcoholic fatty liver disease: The St Francis Heart Study randomized clinical trial. Am. J. Gastroenterol. 2011, 106, 71-77. [CrossRef] [PubMed]

61. Dufour, J.F.; Oneta, C.M.; Gonvers, J.J.; Bihl, F.; Cerny, A.; Cereda, J.M.; Zala, J.F.; Helbling, B.; Steuerwald, M.; Zimmermann, A.; et al. Randomized placebo-controlled trial of ursodeoxycholic acid with vitamin e in nonalcoholic steatohepatitis. Clin. Gastroenterol. Hepatol. 2006, 4, 1537-1543. [CrossRef] [PubMed]

62. Kawanaka, M.; Mahmood, S.; Niiyama, G.; Izumi, A.; Kamei, A.; Ikeda, H.; Suehiro, M.; Togawa, K.; Sasagawa, T.; Okita, M.; et al. Control of oxidative stress and reduction in biochemical markers by Vitamin E treatment in patients with nonalcoholic steatohepatitis: A pilot study. Hepatol. Res. 2004, 29, 39-41. [CrossRef] [PubMed]

63. Tokushige, K.; Hyogo, H.; Nakajima, T.; Ono, M.; Kawaguchi, T.; Honda, K.; Eguchi, Y.; Nozaki, Y.; Kawanaka, M.; Tanaka, S.; et al. Hepatocellular carcinoma in Japanese patients with nonalcoholic fatty liver disease and alcoholic liver disease: Multicenter survey. J. Gastroenterol. 2016, 51, 586-596. [CrossRef] [PubMed]

64. Carbone, L.J.; Angus, P.W.; Yeomans, N.D. Incretin-based therapies for the treatment of non-alcoholic fatty liver disease: A systematic review and meta-analysis. J. Gastroenterol. Hepatol. 2016, 31, 23-31. [CrossRef] [PubMed]

65. Takaki, A.; Kawai, D.; Yamamoto, K. Multiple hits, including oxidative stress, as pathogenesis and treatment target in non-alcoholic steatohepatitis (NASH). Int. J. Mol. Sci. 2013, 14, 20704-20728. [CrossRef] [PubMed]

66. Nguyen, G.; Park, S.Y.; Le, C.T.; Park, W.S.; Choi, D.H.; Cho, E.H. Metformin ameliorates activation of hepatic stellate cells and hepatic fibrosis by succinate and GPR91 inhibition. Biochem. Biophys. Res. Commun. 2018, 495, 2649-2656. [CrossRef] [PubMed]

67. Liu, X.M.; Peyton, K.J.; Shebib, A.R.; Wang, H.; Korthuis, R.J.; Durante, W. Activation of AMPK stimulates heme oxygenase-1 gene expression and human endothelial cell survival. Am. J. Physiol. Heart Circ. Physiol. 2011, 300, H84-H93. [CrossRef] [PubMed]

68. Belfort, R.; Harrison, S.A.; Brown, K.; Darland, C.; Finch, J.; Hardies, J.; Balas, B.; Gastaldelli, A.; Tio, F.; Pulcini, J.; et al. A placebo-controlled trial of pioglitazone in subjects with nonalcoholic steatohepatitis. N. Engl. J. Med. 2006, 355, 2297-2307. [CrossRef] [PubMed]

69. Walter, P.; Schaffhauser, A.O. L-Carnitine, a Vitamin-like Substance for functional food. Ann Nutr. Metabol. 2000, 44, 75-96. [CrossRef] [PubMed]

70. Bril, F.; Kalavalapalli, S.; Clark, V.C.; Lomonaco, R.; Soldevila-Pico, C.; Liu, I.C.; Orsak, B.; Tio, F.; Cusi, K. Response to Pioglitazone in Patients With Nonalcoholic Steatohepatitis with vs. without Type 2 Diabetes. Clin. Gastroenterol. Hepatol. 2018, 16, 558-566. [CrossRef] [PubMed]

71. Bubols, G.B.; Vianna Dda, R.; Medina-Remon, A.; von Poser, G.; Lamuela-Raventos, R.M.; Eifler-Lima, V.L.; Garcia, S.C. The antioxidant activity of coumarins and flavonoids. Mini. Rev. Med. Chem. 2013, 13, 318-334. [PubMed]

72. Loguercio, C.; Festi, D. Silybin and the liver: From basic research to clinical practice. World J. Gastroenterol. 2011, 17, 2288-2301. [CrossRef] [PubMed]

73. Salomone, F.; Barbagallo, I.; Godos, J.; Lembo, V.; Currenti, W.; Cina, D.; Avola, R.; D’Orazio, N.; Morisco, F.; Galvano, F.; et al. Silibinin Restores NAD (+) Levels and Induces the SIRT1/AMPK Pathway in Non-Alcoholic Fatty Liver. Nutrients 2017, 9, 1086. [CrossRef] [PubMed]

74. Wah Kheong, C.; Nik Mustapha, N.R.; Mahadeva, S. A Randomized Trial of Silymarin for the Treatment of Nonalcoholic Steatohepatitis. Clin. Gastroenterol. Hepatol. 2017, 15, 1940-1949. [CrossRef] [PubMed] 
75. Sharma, S.; Black, S.M. Carnitine Homeostasis, Mitochondrial Function, and Cardiovascular Disease. Drug Discov. Today Dis. Mech. 2009, 6, e31-e39. [CrossRef] [PubMed]

76. Ishikawa, H.; Takaki, A.; Tsuzaki, R.; Yasunaka, T.; Koike, K.; Shimomura, Y.; Seki, H.; Matsushita, H.; Miyake, Y.; Ikeda, F.; et al. L-carnitine prevents progression of non-alcoholic steatohepatitis in a mouse model with upregulation of mitochondrial pathway. PLoS ONE 2014, 9, e100627. [CrossRef] [PubMed]

77. Park, S.J.; Park, S.H.; Kim, J.O.; Kim, J.H.; Park, S.J.; Hwang, J.J.; Jin, D.H.; Jeong, S.Y.; Lee, S.J.; Kim, J.C.; et al. Carnitine sensitizes TRAIL-resistant cancer cells to TRAIL-induced apoptotic cell death through the up-regulation of Bax. Biochem. Biophys. Res. Commun. 2012, 428, 185-190. [CrossRef] [PubMed]

78. Huang, H.; Liu, N.; Guo, H.; Liao, S.; Li, X.; Yang, C.; Liu, S.; Song, W.; Liu, C.; Guan, L.; et al. L-carnitine is an endogenous HDAC inhibitor selectively inhibiting cancer cell growth in vivo and in vitro. PLOS ONE 2012, 7, e49062. [CrossRef] [PubMed]

79. Huang, H.; Liu, N.; Yang, C.; Liao, S.; Guo, H.; Zhao, K.; Li, X.; Liu, S.; Guan, L.; Liu, C.; et al. HDAC inhibitor L-carnitine and proteasome inhibitor bortezomib synergistically exert anti-tumor activity in vitro and in vivo. PLoS ONE 2012, 7, e52576. [CrossRef] [PubMed]

80. Li, M.; Zhang, W.; Wang, B.; Gao, Y.; Song, Z.; Zheng, Q.C. Ligand-based targeted therapy: A novel strategy for hepatocellular carcinoma. Int. J. Nanomed. 2016, 11, 5645-5669. [CrossRef] [PubMed]

81. Garcia, E.R.; Gutierrez, E.A.; de Melo, F.; Novaes, R.D.; Goncalves, R.V. Flavonoids Effects on Hepatocellular Carcinoma in Murine Models: A Systematic Review. Evid. Based Complement. Alternat. Med. 2018, 2018, 6328970. [CrossRef] [PubMed]

82. Pan, Z.; Zhuang, J.; Ji, C.; Cai, Z.; Liao, W.; Huang, Z. Curcumin inhibits hepatocellular carcinoma growth by targeting VEGF expression. Oncol. Lett. 2018, 15, 4821-4826. [CrossRef] [PubMed]

83. Liu, H.T.; Huang, Y.C.; Cheng, S.B.; Huang, Y.T.; Lin, P.T. Effects of coenzyme Q10 supplementation on antioxidant capacity and inflammation in hepatocellular carcinoma patients after surgery: A randomized, placebo-controlled trial. Nutr. J. 2016, 15, 85. [CrossRef] [PubMed]

84. Cai, X.; Hu, X.; Cai, B.; Wang, Q.; Li, Y.; Tan, X.; Hu, H.; Chen, X.; Huang, J.; Cheng, J.; et al. Metformin suppresses hepatocellular carcinoma cell growth through induction of cell cycle G1/G0 phase arrest and p21CIP and p27KIP expression and downregulation of cyclin D1 in vitro and in vivo. Oncol. Rep. 2013, 30, 2449-2457. [CrossRef] [PubMed]

85. Tajima, K.; Nakamura, A.; Shirakawa, J.; Togashi, Y.; Orime, K.; Sato, K.; Inoue, H.; Kaji, M.; Sakamoto, E.; Ito, Y.; et al. Metformin prevents liver tumorigenesis induced by high-fat diet in C57Bl/6 mice. Am. J. Physiol. Endocrinol. Metab. 2013, 305, E987-E998. [CrossRef] [PubMed]

86. Sid, B.; Glorieux, C.; Valenzuela, M.; Rommelaere, G.; Najimi, M.; Dejeans, N.; Renard, P.; Verrax, J.; Calderon, P.B. AICAR induces Nrf2 activation by an AMPK-independent mechanism in hepatocarcinoma cells. Biochem. Pharmacol. 2014, 91, 168-180. [CrossRef] [PubMed]

87. Kim, H.; Lee, H.J.; Kim, D.J.; Kim, T.M.; Moon, H.S.; Choi, H. Panax ginseng exerts antiproliferative effects on rat hepatocarcinogenesis. Nutr. Res. 2013, 33, 753-760. [CrossRef] [PubMed]

88. Uchida, D.; Takaki, A.; Ishikawa, H.; Tomono, Y.; Kato, H.; Tsutsumi, K.; Tamaki, N.; Maruyama, T.; Tomofuji, T.; Tsuzaki, R.; et al. Oxidative stress balance is dysregulated and represents an additional target for treating cholangiocarcinoma. Free Radic. Res. 2016, 50, 732-743. [CrossRef] [PubMed]

89. Liu, L.; Cao, Y.; Chen, C.; Zhang, X.; McNabola, A.; Wilkie, D.; Wilhelm, S.; Lynch, M.; Carter, C. Sorafenib blocks the RAF/MEK/ERK pathway, inhibits tumor angiogenesis, and induces tumor cell apoptosis in hepatocellular carcinoma model PLC/PRF/5. Cancer Res. 2006, 66, 11851-11858. [CrossRef] [PubMed]

90. Eso, Y.; Marusawa, H. Novel approaches for molecular targeted therapy against hepatocellular carcinoma. Hepatol. Res. 2018, 48, 597-607. [CrossRef] [PubMed]

91. Abou-Alfa, G.K.; Meyer, T.; Cheng, A.L.; El-Khoueiry, A.B.; Rimassa, L.; Ryoo, B.Y.; Cicin, I.; Merle, P.; Chen, Y.; Park, J.W.; et al. Cabozantinib in Patients with Advanced and Progressing Hepatocellular Carcinoma. N. Eng. J. Med. 2018, 379, 54-63. [CrossRef] [PubMed]

92. Chau, I.; Peck-Radosavljevic, M.; Borg, C.; Malfertheiner, P.; Seitz, J.F.; Park, J.O.; Ryoo, B.Y.; Yen, C.J.; Kudo, M.; Poon, R.; et al. Ramucirumab as second-line treatment in patients with advanced hepatocellular carcinoma following first-line therapy with sorafenib: Patient-focused outcome results from the randomised phase III REACH study. Eur. J. Cancer 2017, 81, 17-25. [PubMed] 
93. Li, J.; Wu, P.W.; Zhou, Y.; Dai, B.; Zhang, P.F.; Zhang, Y.H.; Liu, Y.; Shi, X.L. Rage induces hepatocellular carcinoma proliferation and sorafenib resistance by modulating autophagy. Cell Death Dis. 2018, 9, 225. [CrossRef] [PubMed]

94. Casadei Gardini, A.; Faloppi, L.; De Matteis, S.; Foschi, F.G.; Silvestris, N.; Tovoli, F.; Palmieri, V.; Marisi, G.; Brunetti, O.; Vespasiani-Gentilucci, U.; et al. Metformin and insulin impact on clinical outcome in patients with advanced hepatocellular carcinoma receiving sorafenib: Validation study and biological rationale. Eur. J. Cancer 2017, 86, 106-114. [CrossRef] [PubMed]

95. Wan, J.; Liu, T.; Mei, L.; Li, J.; Gong, K.; Yu, C.; Li, W. Synergistic antitumour activity of sorafenib in combination with tetrandrine is mediated by reactive oxygen species (ROS)/Akt signaling. Br. J. Cancer 2013, 109, 342-350. [CrossRef] [PubMed]

96. El-Khoueiry, A.B.; Sangro, B.; Yau, T.; Crocenzi, T.S.; Kudo, M.; Hsu, C.; Kim, T.Y.; Choo, S.P.; Trojan, J.; Welling, T.H.R.; et al. Nivolumab in patients with advanced hepatocellular carcinoma (CheckMate 040): An open-label, non-comparative, phase 1/2 dose escalation and expansion trial. Lancet 2017, 389, 2492-2502. [CrossRef]

97. Zou, W.; Wolchok, J.D.; Chen, L. PD-L1 (B7-H1) and PD-1 pathway blockade for cancer therapy: Mechanisms, response biomarkers, and combinations. Sci. Transl. Med. 2016, 8, 328rv4. [CrossRef] [PubMed]

98. Chamoto, K.; Chowdhury, P.S.; Kumar, A.; Sonomura, K.; Matsuda, F.; Fagarasan, S.; Honjo, T. Mitochondrial activation chemicals synergize with surface receptor PD-1 blockade for T cell-dependent antitumor activity. Proc. Natl. Acad. Sci. USA 2017, 114, E761-E770. [CrossRef] [PubMed]

99. Moyer, V.A.; Force, U.S.P.S.T. Vitamin, mineral, and multivitamin supplements for the primary prevention of cardiovascular disease and cancer: U.S. Preventive services Task Force recommendation statement. Ann. Int. Med. 2014, 160, 558-564. [CrossRef] [PubMed]

100. Klein, E.A.; Thompson, I.M., Jr.; Tangen, C.M.; Crowley, J.J.; Lucia, M.S.; Goodman, P.J.; Minasian, L.M.; Ford, L.G.; Parnes, H.L.; Gaziano, J.M.; et al. Vitamin E and the risk of prostate cancer: The Selenium and Vitamin E Cancer Prevention Trial (SELECT). JAMA 2011, 306, 1549-1556. [CrossRef] [PubMed]

101. Yae, T.; Tsuchihashi, K.; Ishimoto, T.; Motohara, T.; Yoshikawa, M.; Yoshida, G.J.; Wada, T.; Masuko, T.; Mogushi, K.; Tanaka, H.; et al. Alternative splicing of CD44 mRNA by ESRP1 enhances lung colonization of metastatic cancer cell. Nat. Commun. 2012, 3, 883. [CrossRef] [PubMed]

102. Wada, T.; Ishimoto, T.; Seishima, R.; Tsuchihashi, K.; Yoshikawa, M.; Oshima, H.; Oshima, M.; Masuko, T.; Wright, N.A.; Furuhashi, S.; et al. Functional role of CD44v-xCT system in the development of spasmolytic polypeptide-expressing metaplasia. Cancer Sci. 2013, 104, 1323-1329. [CrossRef] [PubMed]

103. Yoshikawa, M.; Tsuchihashi, K.; Ishimoto, T.; Yae, T.; Motohara, T.; Sugihara, E.; Onishi, N.; Masuko, T.; Yoshizawa, K.; Kawashiri, S.; et al. xCT inhibition depletes CD44v-expressing tumor cells that are resistant to EGFR-targeted therapy in head and neck squamous cell carcinoma. Cancer Res. 2013, 73, 1855-1866. [CrossRef] [PubMed]

104. Trachootham, D.; Alexandre, J.; Huang, P. Targeting cancer cells by ROS-mediated mechanisms: A radical therapeutic approach? Nat. Rev. Drug Discov. 2009, 8, 579-591. [CrossRef] [PubMed]

105. Fujiwara, N.; Nakagawa, H.; Enooku, K.; Kudo, Y.; Hayata, Y.; Nakatsuka, T.; Tanaka, Y.; Tateishi, R.; Hikiba, Y.; Misumi, K.; et al. CPT2 downregulation adapts HCC to lipid-rich environment and promotes carcinogenesis via acylcarnitine accumulation in obesity. Gut 2018, 67, 1493-1504. [CrossRef] [PubMed]

(C) 2018 by the authors. Licensee MDPI, Basel, Switzerland. This article is an open access article distributed under the terms and conditions of the Creative Commons Attribution (CC BY) license (http:/ / creativecommons.org/licenses/by/4.0/). 
Article

\title{
Evaluation of a High Concentrate Omega-3 for Correcting the Omega-3 Fatty Acid Nutritional Deficiency in Non-Alcoholic Fatty Liver Disease (CONDIN)
}

\author{
Derek Tobin ${ }^{1, *} \mathbb{( D}$, Merethe Brevik-Andersen ${ }^{2}$, Yan Qin ${ }^{1}$, Jacqueline K. Innes ${ }^{3}$ \\ and Philip C. Calder ${ }^{3,4}$ \\ 1 BASF AS, NO-1327 Lysaker, Norway; Yan.Qin@basf.com \\ 2 Formerly BASF AS, NO-1327 Lysaker, Norway; merethha@gmail.com \\ 3 Human Development and Health Academic Unit, Faculty of Medicine, University of Southampton, \\ Southampton SO16 6YD, UK; innesjackie@gmail.com (J.K.I.); P.C.Calder@soton.ac.uk (P.C.C.) \\ 4 National Institute for Health Research Southampton Biomedical Research Centre, University Hospital \\ Southampton NHS Foundation Trust and University of Southampton, Southampton SO16 6YD, UK \\ * Correspondence: Derek.Tobin@basf.com; Tel.: +47-22-53-48-50
}

Received: 13 July 2018; Accepted: 16 August 2018; Published: 20 August 2018

\begin{abstract}
This randomized controlled trial investigated the safety and efficacy of MF4637, a high concentrate omega-3 fatty acid preparation, in correcting the omega-3 fatty acid nutritional deficiency in non-alcoholic fatty liver disease (NAFLD). The primary end point of the study was set as the change of red blood cell (RBC) eicosapentaenoic acid (EPA) and docosahexaenoic acid (DHA) by MF4637. Whether the omega-3 concentrate could lower liver fat was evaluated in a subset of patients. Furthermore, 176 subjects with NAFLD were randomized to receive the omega- 3 concentrate $(n=87)$ or placebo $(n=89)$ for 24 weeks, in addition to following standard-of-care dietary guidelines. The omega-3 index, omega-6: omega-3 fatty acid ratio and quantitative measurements of RBC EPA and DHA were determined at baseline and study completion. Magnetic resonance imaging of liver fat was conducted in a subset of patients. Administration of high concentrate omega-3 for 24 weeks significantly increased the omega- 3 index and absolute values of RBC EPA and DHA, and decreased the RBC omega-6: omega-3 fatty acid ratio $(p<0.0001)$. A significant reduction in liver fat content was reported in both groups.
\end{abstract}

Keywords: non-alcoholic fatty liver disease; NAFLD; omega-3 fatty acid; EPA; DHA; omega-3 index

\section{Introduction}

Non-alcoholic fatty liver disease (NAFLD) is the presence of hepatic steatosis ( $>5 \%$ liver fat assessed by imaging modalities or $>5 \%$ of cells containing visible lipid droplets from histology) that is not related to significant alcohol consumption, hereditary disorders, viral infection or steatogenic medication [1]. Early NAFLD is typically reversible, but can develop in some $30 \%$ of cases into non-alcoholic steatohepatitis (NASH), presenting as hepatic steatosis with inflammation, ballooning and evidence of hepatocellular injury with or without fibrosis [1-3]. NAFLD is associated with metabolic risk factors such as obesity, diabetes and dyslipidemia, and its prevalence has risen sharply in line with the rising rates of obesity and diabetes $[1,4,5]$. In Western countries, NAFLD is the leading cause of liver disease [6]. NAFLD is estimated to affect 20-30\% of the general population, with the prevalence increasing to approximately $75 \%$ of patients with obesity or diabetes, and $90-95 \%$ in the morbidly obese [6-9]. The estimated prevalence of NASH is lower, but significant, at $2-3 \%$ of the general population and one-third of the morbidly obese [1,7]. 
Identification of NAFLD patients in a clinical setting is commonly performed due to suspicion from raised liver enzymes and confirmation of hepatic steatosis by ultrasound. More recently, more advanced techniques such as Fibroscan (Echosens) and lipidomic analysis (OWL) have become available at specialised and general practitioner level. A validated algorithm for steatosis risk, called the fatty liver index (FLI), uses clinically available measurements to predict steatosis and to identify populations at risk of developing further liver-related morbidities [10].

NAFLD is increasingly considered as the hepatic manifestation of metabolic syndrome. The metabolic dysregulation occurring during metabolic syndrome often starts with excess peripheral fat and peripheral insulin resistance with hepatic steatosis and hepatic insulin resistance following as secondary events. Hepatic insulin resistance results in increased circulating glucose and very low density lipoproteins (VLDLs) [11]. The resulting hyperglycemia, hyper-triglyceridaemia and lowered HDL-cholesterol are all risk factors for the development of cardiovascular disease (CVD) [1,2,11,12]. In common with metabolic syndrome, NAFLD is associated with increased morbidity and mortality, particularly from CVD $[1,2,12,13]$. NASH also has the potential to develop into liver cirrhosis, from which $30-40 \%$ of patients will die of liver-related causes such as liver failure or hepatocellular carcinoma within a ten-year period [2,3,14]. Despite the increasing prevalence of NAFLD and its associated morbidity and mortality, there is currently no approved drug therapy for its treatment. The World Gastroenterology Organization (WGO) guidelines state that, in addition to pharmacological management of co-morbidities such as diabetes and dyslipidemia, weight loss and increased physical exercise are the most effective ways to reduce liver fat [6]. However, such lifestyle changes are typically difficult to sustain in the long term, creating a significant unmet need for this condition.

There is mounting evidence that long-chain polyunsaturated fatty acids (PUFAs), especially the marine omega-3 fatty acids eicosapentaenoic acid (EPA) and docosahexaenoic acid (DHA), are depleted in patients with NAFLD [15-19]. This may be due to several factors including impairment of the hepatic metabolic pathways responsible for the synthesis of EPA and DHA from their precursors, increased utilization due to lipid peroxidation caused by raised oxidative stress in NALFD, as well as reduced dietary intake $[15,16,20,21]$. Increased levels of omega-3 PUFAs and reduction of the omega-6: omega-3 ratio enables a shift in hepatic fat metabolism away from de novo lipogenesis and towards fatty acid oxidation and secretion, thereby potentially reducing steatosis in NAFLD [17,22-26]. In support of this, a recently published systematic review and meta-analysis of omega-3 fatty acids in NAFLD patients demonstrate statistically and clinically significant consistent reduction in steatosis with approximately $3 \mathrm{~g}$ EPA plus DHA daily [27]. Overall, existing data demonstrate that NAFLD patients have reduced levels of EPA and DHA compared to healthy individuals and that there are beneficial effects on liver steatosis from increased intake of omega-3 PUFAs at approximately $3 \mathrm{~g} /$ day.

The purpose of this study was to investigate the safety and the ability of an omega-3 fatty acid medical food (MF4637; BASF AS, Lysaker, Norway), to correct the omega-3 fatty acid nutritional deficiency present in NAFLD. The hypotheses being tested are that MF4637 will significantly improve the omega-3 index (EPA + DHA in red blood cells (RBCs)) and lower the RBC omega-6: omega-3 fatty acid ratio in patients with NAFLD. The potential for MF4637 to reduce hepatic fat content was evaluated using magnetic resonance imaging-proton density fat fraction (MRI-PDFF) in a subset of patients. Additional post hoc stratification was performed using the FLI.

\section{Materials and Methods}

\subsection{Study Design}

This was a randomized double-blind placebo-controlled study conducted at 21 investigative sites across the U.S. All procedures involving human participants were approved by Quorum Review IRB, Seattle, WA, USA. All participants provided written informed consent. The trial is registered with ID NCT02923804 at the U.S. National Library of Medicine's ClinicalTrials.gov website. 
Participants were recruited based on the suspected diagnosis of NAFLD, confirmed either by diagnostic imaging performed within the previous year, or by abdominal ultrasound performed at screening. Eligibility was determined, after the informed consent process, at screening, which included review of medical history and current medications, measurement of vital signs (height, weight, blood pressure, heart rate and body mass index (BMI)), hemoglobin A1c (HbA1c), thyroid-stimulating hormone (TSH) and liver function testing. Following written informed consent, each participant was centrally randomized 1:1, stratifying by site, marine omega- 3 fatty acid intake ( $\geq 250 \mathrm{mg} /$ day and $<250 \mathrm{mg} /$ day), diabetes and statin use, to receive either the omega-3 concentrate MF4637 or a placebo (olive oil) for 24 weeks. The duration of the study was determined by taking into account the delay of approximately 6 months required for stabilization of the incorporation of EPA and DHA demonstrated in healthy subjects [28] and an analysis of the literature on the use of omega-3 during NAFLD for periods ranging from 6 months to more than one year [27]. Randomization numbers corresponding to predetermined intervention were assigned in a sequential manner to each subject via an Interactive Voice/Web Response System. One hundred and seventy-six subjects were subsequently randomized to receive either MF4637 $(n=87)$ or placebo $(n=89)$ (Figure 1$)$. The modified intention to treat population was defined as all subjects who took at least a 1-day dose of omega-3 fatty acids or placebo and underwent at least 1 post-randomization primary efficacy assessment.

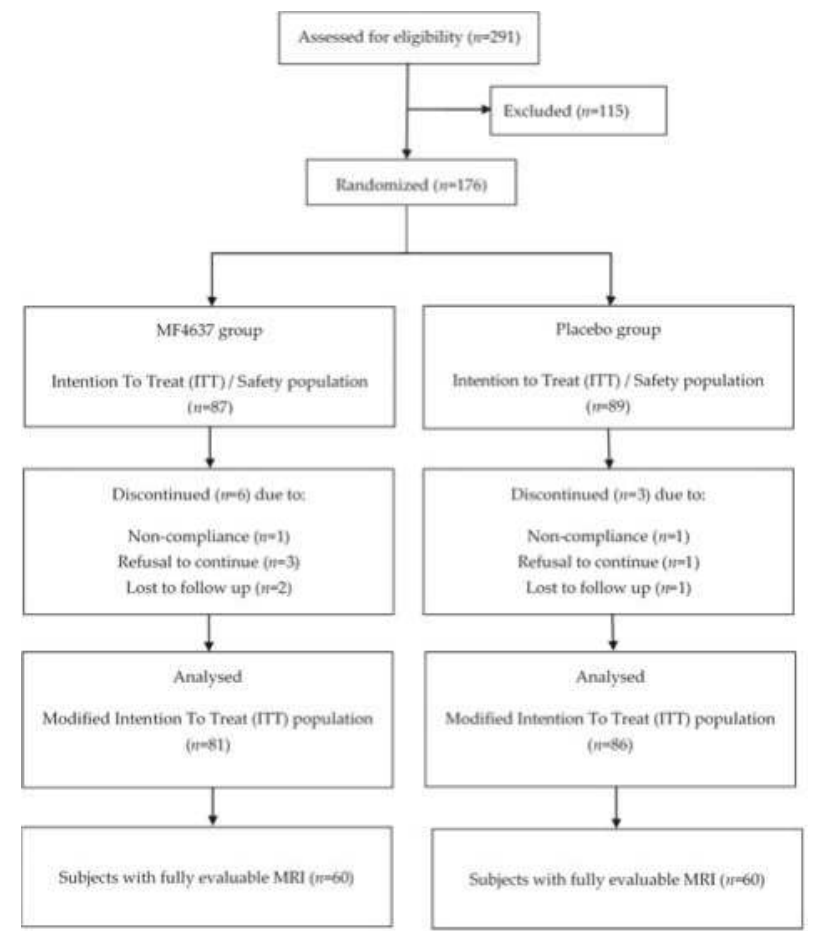

Figure 1. CONSORT flow chart of participant flow.

The omega-3 fatty acid medical food (MF4637; BASF AS, Lysaker, Norway) was provided as soft gel capsules, with each $1 \mathrm{~g}$ capsule containing marine-sourced EPA and DHA as ethyl esters (460 mg and $380 \mathrm{mg}$, respectively). Placebo capsules were identical in size and appearance to MF4637 and contained $1 \mathrm{~g}$ of olive oil. The investigational products were administered in a double-blinded fashion. Study participants were required to take three capsules per day of either MF4637 or placebo with 
food for 24 weeks. Thus, daily intakes of EPA and DHA in the MF4637 group were $1.38 \mathrm{~g}$ and $1.14 \mathrm{~g}$, respectively. Compliance was measured via subject interview and unused capsule counts.

In addition to the investigational product, study participants were advised to reduce normal caloric intake as recommended by the American Association for the Study of Liver Disease (AASLD) standard-of-care guidelines for NAFLD [1], and to maintain stable physical activity levels throughout the study. To provide the American Heart Association (AHA) recommended dietary intake of omega-3 fatty acids [29], participants were required to consume two meals of omega-3 rich fish per week (from a choice of salmon, herring, whitefish, sardines, bluefish and trout) and to reduce foods rich in trans- and omega- 6 fatty acids (fried foods and snacks, fast foods, bacon, turkey bacon, hams, nuts, peanut butter, sesame seeds, sunflower seeds, pumpkin seeds, vegetable oils and margarine (including soybean oil and corn oil), mayonnaise and salad dressing). Dietary intake was monitored regularly throughout the study via participant's food diaries.

At baseline (week 0), week 12 and study completion (week 24), weight, blood pressure, heart rate and BMI were recorded and blood samples collected to assess efficacy (Omega-3 index, RBC omega-6:omega-3 ratio and quantitative measurements of RBC EPA and DHA) and safety (vital signs, standard clinical biochemistry and hematology panels including liver function tests). Adverse events were monitored throughout the study. MRI-PDFF assessments of liver fat were performed at baseline (week 0) and study completion (week 24).

The primary endpoint of the trial was to test the effect of administration with concentrated EPA and DHA on the omega-3 index (RBC EPA + DHA). Secondary endpoints included quantitative measurement of RBC EPA and DHA and assessment of the RBC omega-6: omega-3 ratio. The potential for MF4637 to reduce hepatic fat content was evaluated as an exploratory outcome.

\subsection{Inclusion and Exclusion Criteria}

Selection of the NAFLD study population aimed to include subjects with hepatic steatosis, excluding those with a known previous diagnosis, at any time, of NASH indicating more advanced liver disease. Liver biopsy and histopathology are the only secure means of differentiating NASH from NAFL and therefore the study population may contain NASH patients. Inclusion criteria included age $\geq 18$ years and a recent ( $<1$ year) suspected clinical diagnosis of NAFLD including an imaging modality (e.g., ultrasound). If diagnosis was $>1$ year or an imaging test was absent, an abdominal ultrasound was performed at screening to confirm diagnosis of NAFLD. Other inclusion criteria included not smoking, BMI between $18-39.9 \mathrm{~kg} / \mathrm{m}^{2}$ and, if on statin medication, a history of $>1$ month on a stable dose. Exclusion criteria included a diagnosis of NASH; bilirubin $>2$ times the upper limit of normal; other causes of liver inflammation i.e., hepatitis A, B or C, HIV, cirrhosis, Wilson's disease, autoimmune hepatitis, hemochromatosis, alcoholic steatohepatitis, pancreatitis, or prescription medications known to cause liver toxicity or damage; history of bariatric surgery; significant weight loss ( $>5 \%$ body weight) or rapid weight loss (>1.6 kg/week) within six months of screening; cancer; significant cardiovascular disease including untreated hypertension and significant gastrointestinal, renal, pulmonary, hepatic, biliary or endocrine disease. Furthermore, subjects were excluded if there was significant alcohol consumption; use of any medicine or dietary supplement that may affect NAFLD or lipid metabolism (including omega-3 supplements); use of anti-coagulants; and pregnancy/breastfeeding or sensitivity to any of the study medications or excipients.

\subsection{Measurement of RBC EPA and DHA}

\subsubsection{Quantitative Measurement of RBC EPA and DHA}

Concentrations of total RBC EPA and DHA were measured quantitatively using UPLC-MS/MS. Method validation and all measurements were performed by Diteba Analytical and Bioanalytical Services, A Nutrasource Company (Mississauga, ON, Canada). Blood samples were collected into EDTA vacationer tubes, centrifuged, and plasma and white blood cells (buffy coat) removed. 
The remaining RBCs were washed three times with saline, and $0.5 \mathrm{~mL}$ of the washed packed RBCs added to $1 \mathrm{~mL}$ of distilled water, to which was added $150 \mu \mathrm{L}$ of EDTA/ascorbic acid. The sample was mixed well and stored at $-80^{\circ} \mathrm{C}$ until analyzed.

For the quantitative analytical methodology, a specific amount of standard curve solutions, matrix blanks, quality control samples and thawed study samples were acidified with $\mathrm{HCl}$ and internal standard was added to all tubes except for blanks. Samples were mixed well and incubated at $100^{\circ} \mathrm{C}$ for $45 \mathrm{~min}$, and then cooled to room temperature. Extraction solvent (hexane: dichloromethane: 2-propanol, in a 20:10:1 ratio) was added to each tube, which was mixed well and centrifuged. Capped tubes were submerged in a dry ice-acetone bath to freeze the aqueous layer, and the organic layer in each tube was transferred to another tube. This was evaporated to dryness at $45^{\circ} \mathrm{C}$; then, reconstitution solution was added, mixed thoroughly, and reconstituted samples were transferred into LC-MS vials for injection. The UPLC-MS/MS systems consisted of an Aquity Tandem Quadruple detector, auto-sample manager, binary solvent manager, column manager and Empower 3 data acquisition system. The UPLC column for optimum chromatographic conditions was an Aquity UPLC BEH, C18, $2.1 \mathrm{~mm} \times 50 \mathrm{~mm}$, $1.7 \mu \mathrm{m}$, assembled with a Waters in-line pre-column filter. The mobile phase was a 20:80 mixture of $5 \mathrm{mM}$ ammonium acetate in water and acetonitrile. The injection volume was $5.0 \mathrm{~mL}$, flow rate was $0.30 \mathrm{~mL} / \mathrm{min}$, run time was approximately $2.5 \mathrm{~min}$, column temperature was ambient and sample temperature was $5^{\circ} \mathrm{C} \pm 2{ }^{\circ} \mathrm{C}$. From the resulting chromatograms, EPA and DHA in each sample were calculated by calibration curve using peak area response ratio as response function. The quantitative method provided a range of 1 to $500 \mu \mathrm{g} / \mathrm{mL}$ for EPA, and 5 to $500 \mu \mathrm{g} / \mathrm{mL}$ for DHA.

\subsubsection{Qualitative Measurement of RBC EPA and DHA}

Qualitative measurement of EPA and DHA involved measuring the fatty acid profile of RBCs (consisting of a total of 30 fatty acids) using a gas chromatograph system with an auto sampler and FID detector (Diteba Analytical and Bioanalytical Services, Guelph, ON, Canada). Blood sample collection and processing was identical to that of quantitative analysis of RBC EPA and DHA. A specified amount ( $2 \mathrm{~mL}$ ) of $\mathrm{BF}_{3}-\mathrm{MeOH}$ was added to thawed $\mathrm{RBC}$ samples, mixed, flushed with $\mathrm{N}_{2}$ gas and incubated for $10 \mathrm{~min}$ at $100{ }^{\circ} \mathrm{C}$. After cooling, $250 \mu \mathrm{L}$ of purified water and $750 \mu \mathrm{L}$ of heptane were added to each tube and mixed well. Tubes were centrifuged at 4,000 rpm for $5 \mathrm{~min}$ and the top heptane layer was transferred to another tube and washed with purified water. The top (heptane) layer was transferred to another tube and evaporated to dryness under a stream of $\mathrm{N}_{2}$ gas at $50{ }^{\circ} \mathrm{C}$. Each tube was reconstituted with $10 \mu \mathrm{L}$ of heptane, transferred to a GC vial and flushed with $\mathrm{N}_{2}$ in preparation for injection. For this methodology, the column was a DB Wax, $30 \mathrm{~m} \times 0.25 \mathrm{~mm}$ ID, $0.15 \mu \mathrm{m}$ film or equivalent. The chromatic conditions were a GC with an FID detector, helium carrier gas, an initial oven temperature of $170{ }^{\circ} \mathrm{C}$, increased at $3{ }^{\circ} \mathrm{C} / \mathrm{min}$ to $200{ }^{\circ} \mathrm{C}$, held for $3 \mathrm{~min}$, increased at $2.5^{\circ} \mathrm{C} / \mathrm{min}$ to $225^{\circ} \mathrm{C}$, held for $5 \mathrm{~min}$, then increased at $20^{\circ} \mathrm{C}$ to $245^{\circ} \mathrm{C}$, and then held for $12 \mathrm{~min}$. An external standard was injected three times and then reinjected for every 10 sample injections. From the three consecutive standard injections, an average response factor (RF) for each individual fatty acid was calculated, using the peak area of each individual fatty acid detected.

\subsection{Assessment of Change in Liver Fat}

Assessment of the change in hepatic fat fraction was measured via MRI-PDFF. For each subject, the MRI protocol included a localization sequence and a two-dimensional six-echo spoiled gradient-recalled-echo breath hold sequence. A three-plane localizer followed by a coronal breath-hold localizer was recommended for accurate axial slice prescription. If the scanner was not capable of acquiring six echoes simultaneously, multiple acquisitions with single-echo sequences were performed. From either the six-echo or six single-echo MRI series, the radiologist identified a circular region of interest (ROI) within each of the nine Cournand segments of the liver using the first echo of the series. The radiologist then identified regions with an approximately $2.5 \mathrm{~cm}$ diameter in each of the nine Cournand segments, except for segment 1 (the caudate), in which a region with a diameter of 
approximately $1.5 \mathrm{~cm}$ was identified. The ROI in the caudate was smaller since the caudate is generally too small to identify a region larger than $1.5 \mathrm{~cm}$. The radiologist excluded blood vessels and the periphery of the liver when identifying the ROIs. A fat fraction map was calculated from the six-echo sequence using a multi-interference technique, which took into account the contribution from the individual resonances in the fat spectrum to the observed MRI signal to obtain an accurate estimate of fat. The whole liver hepatic fat fraction (HFF) was expressed as the mean fat fraction across all 9 user-defined ROIs in the liver.

\subsection{Calculation of Fatty Liver Index (FLI) Score}

Fatty liver index (FLI), an algorithm used to predict the presence of hepatic steatosis based on measured values for serum triglycerides (in mg/dL), serum GGT (in IU/L), BMI (in $\mathrm{kg} / \mathrm{m}^{2}$ ) and waist circumference (in $\mathrm{cm}$ ), was calculated using the following equation [10]:

$$
\begin{aligned}
& \quad \mathrm{FLI}=(\mathrm{e} 0.953 \times \text { loge }(\text { triglycerides })+0.139 \times \mathrm{BMI}+0.718 \times \text { loge }(\mathrm{GGT})+0.053 \times \\
& \text { waist circumference- } 15.745) /(1+\mathrm{e} 0.953 \times \text { loge }(\text { triglycerides })+0.139 \times \mathrm{BMI}+0.718 \\
& \quad \times \text { loge }(\mathrm{GGT})+0.053 \times \text { waist circumference- } 15.745) \times 100 \mathrm{P}
\end{aligned}
$$

\subsection{Sample Size Calculations}

It has been reported that the omega- 3 index is $0.5 \%$ higher in healthy subjects compared to those with some form of liver dysfunction, leading to the assumption that a minimum increase of $0.5 \%$ in RBC EPA + DHA may be necessary to achieve nutritional sufficiency in NAFLD patients [30,31]. A conservative between-intervention difference for RBC EPA + DHA, measured as a change from baseline score between standard of care and standard of care plus MF4637, was set at 1.0\% [32] and the standard deviation at 2.0 with a correlation of 0.5 and equal allocation of subjects across the two intervention groups, yielding 64 subjects per intervention arm for a total of 128 . To address the uncertainty of the estimates of intervention effectiveness from the emerging literature, an adaptive blinded mid-course sample size re-estimation procedure was originally planned for the point at which approximately $30 \%$ of the subjects had completed one post-baseline visit (i.e., to Week 12) and had provided the RBC EPA + DHA results (for baseline and Week 12). The sample size re-estimation was performed by one unblinded study statistician. When $30 \%$ of the subjects had provided the Week 24 RBC DHA and EPA data, and the data were considered "lockable" by data management, the data file was exported to a limited access subdirectory, the effect size (change from baseline in plasma level) estimated and the conditional power (CP) were calculated. Because the CP was between $41 \%$ and $90 \%$, the number of subjects per intervention arm was increased, in order to recover the targeted power of $90 \%$. Given that the interim analysis was performed at $30 \%$ of the initial sample size, and the targeted power was $90 \%$, the minimum conditional power cut-off value (CP $\mathrm{min}$ ) was set at $41 \%$. The procedure was performed, as per the Charter, and the recommendation was to increase the sample size to 75 subjects per intervention (i.e., 150 subjects). The actual number of participants recruited to the study was 176 .

\subsection{Statistical Analysis}

The primary outcome (RBC EPA + DHA) was analyzed using a repeated analysis of covariance (ANCOVA) with the stratification factors as covariates, in order to compare the changes in the combined $\mathrm{EPA}+\mathrm{DHA}$ outcome between the two intervention groups (MF4637 group and placebo) across the study. Stratification factors were baseline omega-3 intake, diabetes status and statin use. Additional outcomes were analyzed using the same ANCOVA model applied to the primary outcome: RBC EPA, RBC DHA and the omega-6: omega-3 ratio. All programming was performed in SAS version 9.2 (SAS Institute, Cary, NC, USA) or higher under the Windows Server 2008R2 operating system. 
A regression analysis for absolute RBC EPA + DHA and change in liver fat for the MF4637 group and placebo was planned without testing of statistical significance. Similarly, regression analysis of changes in omega-3 index against baseline omega-3 index was performed. Post hoc analysis of the change in liver fat for intervention and placebo groups after stratification using the baseline Fatty Liver Index was tested using ANCOVA.

\section{Results}

Of the 176 participants that underwent randomization, safety was assessed for 87 subjects in the intervention group and 89 in the placebo group (Figure 1). Of those participants randomized, 167 (81 in the MF4637 group and 86 in the placebo group) were included in the modified intention to treat (mITT) primary outcome analysis.

Baseline anthropometric and biochemical variables of participants randomized to the placebo and MF4637 groups are detailed in Table 1. Of note is the higher mean fasting insulin concentration in the placebo group, which, together with the comparable mean fasting glucose concentration, suggests a likelihood that the placebo group was more insulin resistant than the MF4637 group at study entry.

Table 1. Baseline anthropometric and biochemical variables of participants randomized to placebo and MF4637 groups.

\begin{tabular}{|c|c|c|c|}
\hline Variables & Placebo $^{1}$ & MF4637 2 & $p$-Value * \\
\hline Age, years & $55.1(10.9)$ & $55.3(13.3)$ & 0.93 \\
\hline Sex, M/F & $44 / 42$ & $36 / 45$ & 0.39 \\
\hline Weight, kg & $90.1(18.8)$ & $88.4(18.4)$ & 0.55 \\
\hline Waist circumference, $\mathrm{cm}$ & $105.9(13.1)$ & $106.3(13.2)$ & 0.87 \\
\hline Hip circumference, $\mathrm{cm}$ & $110.5(12.1)$ & $110.9(11.8)$ & 0.85 \\
\hline Waist to hip ratio & $0.96(0.09)$ & $0.96(0.08)$ & 0.95 \\
\hline $\mathrm{BMI}, \mathrm{kg} / \mathrm{m}^{2}$ & $32.4(5.0)$ & $32.1(4.8)$ & 0.59 \\
\hline Systolic blood pressure, mm Hg & $127.0(10.7)$ & $128.0(11.9)$ & 0.64 \\
\hline Diastolic blood pressure, $\mathrm{mm} \mathrm{Hg}$ & $80.3(7.2)$ & $79.8(7.6)$ & 0.33 \\
\hline Heart rate, beats $/ \mathrm{min}$ & $74.7(8.7)$ & $73.2(9.1)$ & 0.25 \\
\hline Statin use, $\%$ & 34.9 & 30.9 & 0.58 \\
\hline Diabetes, \% & 39.5 & 35.0 & 0.97 \\
\hline Fasting glucose, mg/dL & $120.1(48.5)^{2}$ & $119.4(38.1)^{3}$ & 0.97 \\
\hline Fasting insulin, $\mu \mathrm{IU} / \mathrm{mL}$ & $30.2(41.3)$ & $20.8(18.2)^{4}$ & 0.04 \\
\hline $\mathrm{HbA1c}, \%$ & $6.5(1.5)^{5}$ & $6.3(1.4)^{6}$ & 0.20 \\
\hline Triglycerides, mg/dL & $199.1(123.0)^{7}$ & $192.0(125.1)^{4}$ & 0.70 \\
\hline BUN, mg/dL & $14.7(4.9)^{7}$ & $15.4(5.0)^{4}$ & 0.24 \\
\hline Creatinine, $\mathrm{mg} / \mathrm{dL}$ & $0.8(0.2)^{7}$ & $0.3(0.2)^{4}$ & 0.83 \\
\hline $\mathrm{TSH} \mu \mathrm{IU} / \mathrm{mL}$ & $1.9(1.0)^{7}$ & $1.7(0.9)^{8}$ & 0.17 \\
\hline Hs-CRP, mg/L & $6.4(9.2)^{7}$ & $8.1(17.5)$ & 0.61 \\
\hline Albumin, g/dL & $4.29(0.3)^{7}$ & $4.29(0.3)^{4}$ & 0.91 \\
\hline ALT, IU/L & $35.6(24.0)$ & $37.5(39.0)$ & 0.40 \\
\hline AST, IU/L & $25.8(12.2)$ & $27.1(20.3)$ & 0.79 \\
\hline ALP, IU/L & $81.5(31.0)$ & $85.5(45.3)$ & 0.62 \\
\hline GGT, IU/L & $47.1(49.0)$ & $62.2(151.4)$ & 0.78 \\
\hline Bilirubin, mg/dL & $0.5(0.2)$ & $0.5(0.3)$ & 0.91 \\
\hline
\end{tabular}

1 Data for $n=86$ participants unless otherwise specified. ${ }^{2}$ Data for $n=81$ participants unless otherwise specified. ${ }^{3}$ Data for $n=75$ participants. ${ }^{4}$ Data for $n=80$ participants. ${ }^{5}$ Data for $n=83$ participants. ${ }^{6}$ Data for $n=79$ participants. ${ }^{7}$ Data for $n=85$ participants. ${ }^{8}$ Data for $n=78$ participants. Values expressed as Mean (SD). Abbreviations: ALP, alkaline phosphatase; ALT, alanine aminotransferase; AST, aspartate aminotransferase; BMI, body mass index; BUN, blood urea nitrogen; GGT, gamma-glutamyl transferase; HbA1c, glycated haemoglobin; hs-CRP, high-sensitivity C-reactive protein; TSH, thyroid stimulating hormone. * All statistical tests performed were $t$-tests except for chi-square tests for the following variables: Sex; Statin use and Diabetes, and Wilcoxon two-sample tests for the following variables: Fasting glucose; HbA1c; ALP and GGT.

Table 2 details the main anthropometric and biochemical variables for participants randomized to the placebo and MF4637 groups at baseline and after 24 weeks of intervention. The overall weight of subjects in either intervention group remained unchanged from study start to study end. Triglyceride 
levels at baseline were similar in both groups and would be clinically regarded as borderline raised. At the end of the study, a statistically significant reduction in triglycerides was only seen in the MF4637 group. Interestingly, the liver enzymes ALT, AST and GGT showed statistical reductions at the end of study in the placebo group and not in the MF4637 group. This implies an overall improvement in liver function without weight loss.

Compliance regarding the investigational products was $89 \%$ in the MF4637 group and $91 \%$ in the placebo group. There were no serious adverse events related to study interventions reported during the 24-week study. Mild incidences of eructation $(n=1)$, dysgeusia $(n=1)$, abdominal bloating $(n=$ $1)$ and increased blood triglycerides $(n=1)$ together with a moderate case of diarrhea $(n=1)$ were reported in the MF4637 group and suspected to be related. One participant in the MF4637 group and two participants in the placebo group discontinued the study due to adverse events.

Table 2. Anthropometric and biochemical variables of participants randomized to placebo and MF4637 groups at baseline and study completion.

\begin{tabular}{|c|c|c|c|c|c|c|c|}
\hline \multirow[b]{2}{*}{ Variables } & \multicolumn{3}{|c|}{ Placebo $^{1}$} & \multicolumn{3}{|c|}{ MF4637 2} & \multirow{2}{*}{$\begin{array}{l}\text { Two-Sample Test for } \\
\text { Change from } \\
\text { Baseline } p \text {-Value }\end{array}$} \\
\hline & Baseline & End of Study & $p$-Value * & Baseline & $\begin{array}{l}\text { End of } \\
\text { Study }\end{array}$ & $p$-Value * & \\
\hline Age, years & $55.1(10.9)$ & & & $55.3(13.3)$ & & & \\
\hline Sex, M/F & $44 / 42$ & & & $36 / 45$ & & & \\
\hline Weight, $\mathrm{kg}$ & $90.1(18.8)$ & $89.5(18.5)$ & 0.84 & $88.4(18.4)$ & $89.0(18.8)$ & 0.082 & 0.23 \\
\hline Waist circumference, $\mathrm{cm}$ & $105.9(13.1)$ & $104.7(13.9)$ & 0.005 & $106.3(13.2)$ & $105.5(12.2)$ & 0.72 & 0.18 \\
\hline Hip circumference, $\mathrm{cm}$ & $110.5(12.1)$ & $110.0(11.7)$ & 0.31 & $110.9(11.8)$ & $110.4(11.9)$ & 0.81 & 0.42 \\
\hline Waist to hip ratio & $0.96(0.1)$ & $0.95(0.1)$ & 0.081 & $0.96(0.08)$ & $0.96(0.1)$ & 0.66 & 0.58 \\
\hline BMI, $\mathrm{kg} / \mathrm{m}^{2}$ & $32.4(5.0)$ & $32.3(4.8)$ & 0.74 & $32.1(4.8)$ & $32.3(5.0)$ & 0.078 & 0.18 \\
\hline SBP, mm Hg & $127.0(10.7)$ & $128.8(15.0)$ & 0.23 & $128.0(11.9)$ & $126.6(10.6)$ & 0.29 & 0.11 \\
\hline DBP, $\mathrm{mm} \mathrm{Hg}$ & $80.3(7.2)$ & $79.9(9.9)$ & 0.25 & $79.8(7.6)$ & $77.8(8.4)$ & 0.032 & 0.53 \\
\hline Heart rate, beats/min & $74.7(8.7)$ & $74.1(8.2)$ & 0.59 & $73.2(9.1)$ & $74.2(9.8)$ & 0.17 & 0.17 \\
\hline Statin use, $\%$ & 34.9 & & & 30.9 & & & \\
\hline Diabetes, $\%$ & 39.5 & & & 35.0 & & & \\
\hline Fasting glucose, $\mathrm{mg} / \mathrm{dL}$ & $120.1(48.5)^{3}$ & $125.4(56.8)^{3}$ & 0.39 & $119.4(38.1)^{3}$ & $127.5(55.2)^{3}$ & 0.0616 & 0.38 \\
\hline Fasting insulin, $\mu \mathrm{IU} / \mathrm{mL}$ & $30.2(41.3)^{4}$ & $30.1(35.8)^{4}$ & 0.51 & $20.8(18.2)^{5}$ & $24.0(24.3)^{5}$ & 0.21 & 0.63 \\
\hline $\mathrm{HbA} 1 \mathrm{c}, \%$ & $6.5(1.5)^{6}$ & $6.6(1.7)^{6}$ & 0.67 & $6.3(1.4)^{7}$ & $6.3(1.4)^{7}$ & 0.83 & 0.87 \\
\hline Triglycerides, mg/dL & $199.1(123.0)^{2}$ & $185.7(118.0)^{2}$ & 0.52 & $192.0(125.1)^{5}$ & $157.8(84.2)^{5}$ & 0.0008 & 0.053 \\
\hline $\mathrm{BUN}, \mathrm{mg} / \mathrm{dL}$ & $14.7(4.9)^{2}$ & $15.0(4.5)^{2}$ & 0.88 & $15.4(5.0)^{5}$ & $16.2(4.9)^{5}$ & 0.25 & 0.46 \\
\hline Creatinine, $\mathrm{mg} / \mathrm{dL}$ & $0.8(0.2)^{2}$ & $0.82(0.2)^{2}$ & 0.51 & $0.3(0.2)^{5}$ & $0.84(0.2)^{5}$ & 0.26 & 0.19 \\
\hline $\mathrm{TSH} \mu \mathrm{IU} / \mathrm{mL}$ & $1.9(1.0)^{2}$ & $2.5(2.7)^{2}$ & 0.025 & $1.7(0.9)^{7}$ & $1.8(0.9)^{7}$ & 0.043 & 0.86 \\
\hline Hs-CRP, mg/L & $6.4(9.2)^{2}$ & $5.5(5.8)^{2}$ & 0.46 & $8.1(17.5)^{8}$ & $6.7(10.9)^{8}$ & 0.75 & 0.82 \\
\hline Albumin, g/dL & $4.29(0.3)^{2}$ & $4.3(0.3)^{2}$ & 0.69 & $4.29(0.3)^{5}$ & $4.3(0.3)^{5}$ & 0.62 & 0.91 \\
\hline ALT, IU/L & $35.6(24.0)$ & $29.8(21.2)$ & 0.005 & $37.5(39.0)$ & $38.1(37.7)$ & 0.48 & 0.015 \\
\hline AST, IU/L & $25.8(12.2)$ & $23.9(13.5)$ & 0.036 & $27.1(20.3)$ & $28.7(24.6)$ & 0.37 & 0.036 \\
\hline ALP, IU/L & $81.5(31.0)$ & $76.3(21.5)$ & 0.01 & $85.5(45.3)$ & $83.2(40.3)$ & 0.09 & 0.73 \\
\hline GGT, IU/L & $47.1(49.0)$ & $37.1(32.2)$ & $<.0001$ & $62.2(151.4)$ & 57.1 (108.7) & 0.37 & 0.058 \\
\hline Bilirubin, $\mathrm{mg} / \mathrm{dL}$ & $0.5(0.2)$ & $0.47(0.2)$ & 0.70 & $0.5(0.3)$ & $0.5(0.2)$ & 0.88 & 0.72 \\
\hline
\end{tabular}

${ }^{1}$ Data for $n=86$ participants unless otherwise specified. ${ }^{2}$ Data for $n=81$ participants unless otherwise specified. ${ }^{3}$ Data for $n=72$ participants. ${ }^{4}$ Data for $n=82$ participants. ${ }^{5}$ Data for $n=78$ participants. ${ }^{6}$ Data for $n=74$ participants. ${ }^{7}$ Data for $n=76$ participants. ${ }^{8}$ Data for $n=79$ participants. Values are expressed as Mean (SD). Abbreviations: ALP, alkaline phosphatase; ALT, alanine aminotransferase; AST, aspartate aminotransferase; BMI, body mass index; BUN, blood urea nitrogen; DBP, diastolic blood pressure; GGT, gamma-glutamyl transferase; $\mathrm{HbA1c}$, glycated haemoglobin; hs-CRP, high-sensitivity C-reactive protein; SBP, systolic blood pressure.* Within-group differences were assessed using paired $t$-tests or Wilcoxon signed rank tests. \# Intergroup differences were assessed using two-sample $t$-tests or two-sample Wilcoxon tests.

\subsection{Effect of Intervention on Omega-3 Index}

The baseline omega-3 index was similar for placebo and intervention groups. Compared to placebo, the mean omega-3 index increased significantly from $4.8 \%$ to $8.0 \%$ at study completion in the MF4637 group, representing a mean $3.2 \%$ change from baseline $(p<0.0001)$ (Table 3$)$. In the placebo group, the omega-3 index increased slightly from $4.9 \%$ at baseline to $5.3 \%$ at study completion, 
representing a mean change of $0.4 \%$. Regression analysis of the data for participants in the MF4637 group suggests that the change in omega-3 index was inversely related to the baseline omega- 3 index, with lower baseline values resulting in greater increases by the end of the 24-week intervention (data not shown [33]).

Table 3. RBC fatty acid content at baseline and after 12- and 24-week intervention with placebo or MF4637.

\begin{tabular}{|c|c|c|c|c|c|c|c|c|c|}
\hline & \multicolumn{4}{|c|}{ Placebo $(n=86)$} & \multicolumn{4}{|c|}{ MF4637 $(n=81)$} & \multirow[b]{2}{*}{$p$-Value } \\
\hline & Baseline & $\begin{array}{l}T=12 \\
\text { weeks }\end{array}$ & $\begin{array}{l}\mathrm{T}=24 \\
\text { weeks }\end{array}$ & $\begin{array}{l}\text { Change } \\
\text { from } \\
\text { Baseline }\end{array}$ & Baseline & $\begin{array}{c}\mathrm{T}=12 \\
\text { weeks }\end{array}$ & $\begin{array}{l}\mathrm{T}=24 \\
\text { weeks }\end{array}$ & $\begin{array}{c}\text { Change } \\
\text { from } \\
\text { Baseline }\end{array}$ & \\
\hline $\begin{array}{l}\text { RBC omega-3 } \\
\text { index, } \%\end{array}$ & $4.9(1.2)$ & $5.8(1.3)$ & $5.3(1.1)$ & $0.4(1.0)$ & $4.8(1.1)$ & $8.7(2.3)$ & $8.0(2.6)$ & $3.2(2.7)$ & $<0.0001$ \\
\hline $\begin{array}{l}\text { RBC EPA + DHA, } \\
\mu \mathrm{g} / \mathrm{mL}\end{array}$ & $32.3(26.4)$ & $34.9(21.0)$ & $33.1(20.5)$ & $1.2(14.9)$ & $29.6(17.5)$ & $51.5(38.9)$ & $52.9(40.7)$ & $21.2(28.7)$ & $<0.0001$ \\
\hline RBC EPA, \% & $0.54(0.3)$ & $0.54(0.2)$ & $0.54(0.2)$ & $0.002(0.3)$ & $0.53(0.2)$ & $1.6(0.9)$ & $1.4(0.9)$ & $0.9(1.0)$ & $<0.0001$ \\
\hline $\mathrm{RBC}$ EPA, $\mu \mathrm{g} / \mathrm{mL}$ & $3.8(6.6)$ & $3.7(2.6)$ & $4.1(5.7)$ & $0.4(7.0)$ & $3.0(2.6)$ & $10.4(10.7)$ & $10.6(12.0)$ & $7.1(10.5)$ & $<0.0001$ \\
\hline RBC DHA, \% & $4.3(1.1)$ & $5.2(1.2)$ & $4.8(1.0)$ & $0.4(0.9)$ & $4.3(1.0)$ & $7.1(1.5)$ & $6.6(1.8)$ & $2.3(1.9)$ & $<0.0001$ \\
\hline $\mathrm{RBC} D H A, \mu \mathrm{g} / \mathrm{mL}$ & $28.5(21.0)$ & $31.2(18.6)$ & $29.0(16.2)$ & $0.7(10.7)$ & $26.6(15.3)$ & $41.0(28.8)$ & $42.4(29.8)$ & $14.1(19.5)$ & $<0.0001$ \\
\hline $\begin{array}{l}\text { RBC omega- } 6 \text { : } \\
\text { omega-3 }\end{array}$ & $4.9(1.1)$ & $4.5(0.9)$ & $4.7(0.8)$ & $-0.2(0.7)$ & $4.9(1.2)$ & $3.0(0.9)$ & $3.3(1.5)$ & $-1.6(1.8)$ & $<0.0001$ \\
\hline
\end{tabular}

\subsection{Effect of Intervention on RBC EPA + DHA, EPA and DHA Values}

Absolute RBC EPA + DHA increased on average from $29.6 \mu \mathrm{g} / \mathrm{mL}$ at baseline to $52.9 \mu \mathrm{g} / \mathrm{mL}$ at study completion (representing a significant increase of $21.2 \mu \mathrm{g} / \mathrm{mL}$ ) in the MF4637 group, compared to a $1.2 \mu \mathrm{g} / \mathrm{mL}$ increase from baseline in the placebo group (significance between groups, $p<0.0001$ ) (Table 3). In terms of absolute values of EPA and DHA separately, RBC EPA increased by a significant $7.1 \mu \mathrm{g} / \mathrm{mL}$ to $10.6 \mu \mathrm{g} / \mathrm{mL}$ at study completion in the MF4637 group versus a $0.4 \mu \mathrm{g} / \mathrm{mL}$ increase in the placebo group $(p<0.0001)$ (Table 3). RBC DHA increased by more than EPA, with a mean of $14.1 \mu \mathrm{g} / \mathrm{mL}$ increase to $42.4 \mu \mathrm{g} / \mathrm{mL}$ in the MF4637 group versus a $0.7 \mu \mathrm{g} / \mathrm{mL}$ increase in the placebo group $(p<0.0001)$ (Table 3$)$.

Regarding the percentage of individual EPA and DHA as a proportion of total RBC fatty acids, both parameters increased significantly in the MF4637 group compared to placebo (Table 3). Specifically, RBC EPA as a percentage of total fatty acids increased by a significant $0.9 \%$ to $1.4 \%$ in the MF4637 group compared to a $0.002 \%$ increase in the placebo group $(p<0.0001)$. RBC DHA as a percentage of total fatty acids increased by a greater proportion than EPA, resulting in a $2.3 \%$ increase to $6.6 \%$ in the MF4637 group versus $0.4 \%$ increase in the placebo group $(p<0.0001)$.

\subsection{Effect of Intervention on RBC Omega-6: Omega-3 Ratio}

RBC omega-6: omega-3 ratio was not different between the two groups at baseline. Following administration of MF4637 for 24 weeks, the RBC omega-6: omega-3 ratio decreased by a mean of 1.6 from 4.9 at baseline to 3.3 at study completion, compared to a 0.2 decrease to 4.7 in the placebo group $(p<0.0001)$ (Table 3).

\subsection{Effect of Intervention on Liver Fat}

In the mITT analysis of liver fat content, 120 participants (60 in each trial arm) completed both the baseline and end of study MRI-PDFF assessment. In this population, baseline liver fat was $17.4 \%$ in the placebo group and $14.4 \%$ in the MF4637 group $(p=0.0689)$. Both the MF4637 and placebo groups demonstrated a decrease in liver fat percentage ( $26 \%$ and $28 \%$, respectively), (Table 4 ). As such, there was no statistically significant difference in the decrease in liver fat between the groups. 
Table 4. MRI-PDFF liver fat percentage at baseline and after 24-week intervention with placebo or MF4637.

\begin{tabular}{|c|c|c|c|c|c|c|c|c|c|}
\hline & \multicolumn{4}{|c|}{ Placebo $^{1}$} & \multicolumn{4}{|c|}{${\text { MF } 4637^{1}}^{1}$} & \multirow{3}{*}{$p$-Valu } \\
\hline & \multirow{2}{*}{$\begin{array}{c}\mathrm{T}=0 \\
\text { weeks }\end{array}$} & \multirow{2}{*}{$\begin{array}{l}\mathrm{T}=24 \\
\text { weeks }\end{array}$} & \multicolumn{2}{|c|}{ Change from Baseline } & \multirow{2}{*}{$\begin{array}{c}\mathrm{T}=0 \\
\text { weeks }\end{array}$} & \multirow{2}{*}{$\begin{array}{c}\mathrm{T}=24 \\
\text { weeks }\end{array}$} & \multicolumn{2}{|c|}{ Change from Baseline } & \\
\hline & & & Absolute & Relative & & & Absolute & Relative & \\
\hline
\end{tabular}

${ }^{1}$ As assessed for modified ITT population (Placebo, $n=60$; MF4637, $n=60$ ). Values expressed as Mean (SD); T: Time.

\subsection{Relationship between RBC EPA +DHA Enrichment and Liver Fat Content}

Regression analysis of the data by intervention group suggests that the change from baseline in liver fat percentage was inversely related to the change in absolute RBC EPA + DHA values in the MF4637 group. Thus, the largest decreases in liver fat were observed in participants with the greatest increases in absolute RBC EPA + DHA (Figure 2). Hence, whilst there was no significant difference between MF4637 and placebo with regard to overall reduction of liver fat, there was an association between increasing RBC EPA+DHA enrichment and decreasing percentage liver fat content.

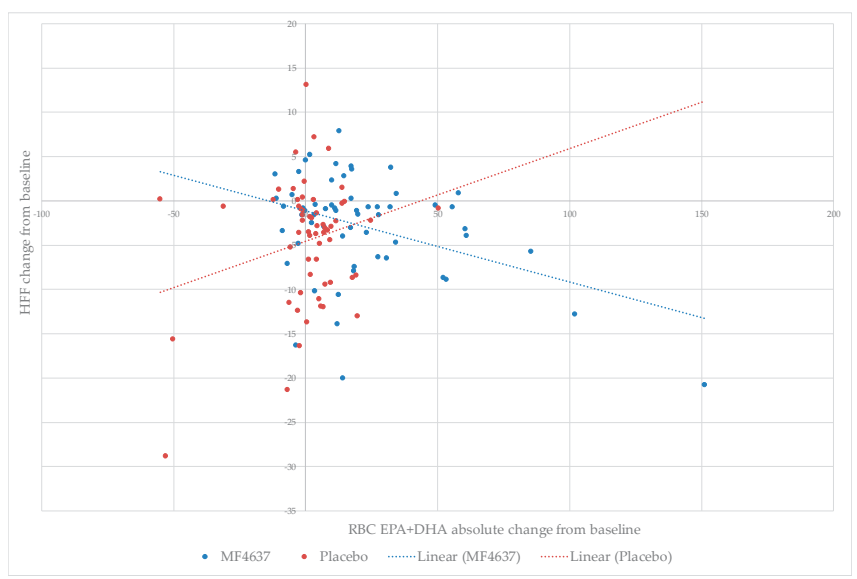

Figure 2. Relationship between change in absolute RBC EPA + DHA and change in liver fat.

\subsection{Relationship between Baseline Fatty Liver Index (FLI) and Change in Liver Fat Content}

Post hoc analysis of the MF4637 group utilizing ANCOVA with baseline fatty liver index (FLI) as covariate found that, in those patients with higher baseline FLI scores (indicative of more probable fatty liver), there was a greater reduction in liver fat compared to placebo (Table 5). Following 24 weeks of intervention with MF4637, patients with baseline FLI $\geq 40(n=17)$ had a placebo corrected, statistically significant $44 \%$ relative decrease in liver fat content $(p=0.009)$. This equates to a $7.45 \%$ absolute decrease in placebo corrected liver fat content for the MF4637 group. 
Table 5. Change in MRI-PDFF liver fat percentage after 24-week intervention with MF4637 stratified by baseline FLI score.

\begin{tabular}{|c|c|c|c|c|c|c|c|}
\hline & & \multirow{2}{*}{$\begin{array}{l}\text { Baseline HFF } \\
\text { Mean (SD) }\end{array}$} & \multirow{2}{*}{$\begin{array}{l}\text { Study End HFF } \\
\text { Mean (SD) }\end{array}$} & \multicolumn{4}{|c|}{ Change in MRI-PDFF Liver Fat Percentage (\%) } \\
\hline & & & & Absolute Change & $p$-Value ${ }^{1}$ & Relative Change & $p$-Value ${ }^{1}$ \\
\hline \multirow{2}{*}{ FLI $<30^{2}$} & Placebo & $15.6(11.2)$ & $11.2(7.8)$ & \multirow{2}{*}{$2.25(1.39)$} & \multirow{2}{*}{0.11} & \multirow{2}{*}{$2.7(11.3)$} & \multirow{2}{*}{0.81} \\
\hline & Omega-3 & $12.5(8.6)$ & $10.3(7.7)$ & & & & \\
\hline \multirow{2}{*}{$\mathrm{FLI} \geq 30^{3}$} & Placebo & $20.6(9.4)$ & $16.0(7.9)$ & \multirow{2}{*}{$-2.47(2.53)$} & \multirow{2}{*}{0.34} & \multirow{2}{*}{$-9.3(15.3)$} & \multirow{2}{*}{0.55} \\
\hline & Omega-3 & $19.3(11.1)$ & $13.5(7.0)$ & & & & \\
\hline \multirow{2}{*}{ FLI $\geq 40^{4}$} & Placebo & $20.2(9.5)$ & $16.3(8.4)$ & \multirow{2}{*}{$-7.45(2.81)$} & \multirow{2}{*}{0.02} & \multirow{2}{*}{$-44.1(14.6)$} & \multirow{2}{*}{0.009} \\
\hline & Omega-3 & $20.7(10.4)$ & $10.6(5.2)$ & & & & \\
\hline
\end{tabular}

${ }^{1} p$-value is for the mean percentage change from baseline to 24 weeks (placebo corrected) using ANCOVA. ${ }^{2}$ Data for $n=89$ participants $(n=43$ placebo; $n=46 \mathrm{MF} 4637)$. ${ }^{3}$ Data for $n=28$ participants $(n=16$ placebo; $n=12$ MF4637). ${ }^{4}$ Data for $n=17$ participants ( $n=12$ placebo; $\left.n=5 \mathrm{MF} 4637\right)$. Values expressed as Mean (SD). Abbreviations: FLI, Fatty Liver Index; HFF, Hepatic Fat Fraction.

\subsection{Relationship between RBC EPA + DHA Enrichment and Liver Enzymes AST and ALT}

At study entry, the mean baseline concentrations of the liver enzymes AST and ALT were within the normal range for both the placebo and MF4637 groups. This is not surprising considering that liver enzymes may be normal in up to $80 \%$ of NAFLD patients [34]. Similar to the relationship between RBC $\mathrm{EPA}+\mathrm{DHA}$ enrichment and change in liver fat discussed above, a non-statistical inverse association was also found between the change in absolute RBC EPA + DHA and change in the concentrations of the liver enzymes AST and ALT in the intervention group. Thus, with increasing change in absolute RBC EPA + DHA, there were greater decreases in both AST and ALT concentrations (data not shown). However, there were significant reductions in AST, ALT and GGT in the placebo group (unrelated to omega-3 measurements), suggesting that multiple factors may be impacting the clinical outcome of the study groups.

\subsection{Effect of Intervention on Plasma Triglycerides}

At study completion, plasma triglycerides (Table 2) decreased by a statistically significant $18 \%$ from baseline values in the intervention group $(p=0.0008)$ compared to a $7 \%$ reduction in triglycerides from baseline values in the placebo group ( $p=0.52$; for placebo adjusted effect of M4637 $p=0.053$ ). The baseline levels for triglycerides were only moderately increased compared to normality and would clinically be defined as "borderline high".

\section{Discussion}

This study demonstrates that intervention with high concentrate omega-3 for 24 weeks significantly raises the omega- 3 index and decreases the omega-6: omega- 3 fatty acid ratio in adults with NAFLD. Furthermore, the EPA and DHA enrichment achieved with intervention was significantly greater than that obtained by dietary recommendation alone. This is of importance, considering the depleted omega-3 status of NAFLD patients [15-19] and the current lack of therapeutic options for the treatment of NAFLD other than lifestyle recommendation [6]. Furthermore, the metabolic efficacy of the high concentrate omega- 3 was confirmed through its significant lowering of plasma triglyceride levels compared to baseline levels.

When assessing the mITT population from whom baseline and post-intervention data from MRI-PDFF were available, intervention with both a high concentrate omega-3 and placebo caused a significant reduction in hepatic steatosis that was significant within each of the groups. There may be several reasons for the liver fat-lowering effect observed in the placebo group. All study participants were required to follow the standard-of-care dietary recommendations for the management of NAFLD. This included adherence to a diet with reduced caloric intake, and increased omega- 3 and reduced omega- 6 and trans-fatty acid consumption. Hence, participants in the placebo group may have 
achieved a decrease in liver fat percentage from the effects of these dietary recommendations alone, particularly from increased omega- 3 fatty acid intake from the diet. However, it should be remembered that the intervention group had a greater increase in omega- 3 index than placebo, suggesting a minimal influence from dietary changes. Similar findings of liver fat improvement in the placebo group have been reported in several other studies. These studies propose that MRI-PDFF volatility in early NAFLD subjects may contribute to data variability [35].

Of general note in the current study is the relatively low baseline liver fat by MRI-PDFF (mean $17 \%$ and $14 \%$ in placebo and intervention groups, respectively), which, together with relatively low baseline AST and ALT levels, indicate an early stage of NAFLD in this study population. Early stages of NAFLD are characterized by changeable liver fat content that can be affected by factors such as high-fat meals. This is in contrast to advanced NAFLD, in which the liver fat is likely to be more stable and less influenced by such factors.

A further confounding factor may be the high number (over one-third) of diabetic participants, and the number of subjects taking metformin and thiazolidinedione during the trial. From the mean baseline fasting insulin and glucose concentrations, the placebo group is also likely to have been more insulin resistant than the intervention group at study entry. These factors may have had some effect on liver fat metabolism. Indeed, on stratification of the data by diabetes status, those with diabetes had a greater reduction in liver fat from baseline (mean decrease of $4.9 \%$ in MF4637 group versus $6.3 \%$ decrease in placebo group) compared to those that did not have diabetes (mean decrease of $1.6 \%$ in MF4637 group versus 3.3\% decrease in placebo group). To date, there have been very few trials conducted in the diabetic NAFLD population. One limitation of this study is the lack of additional lifestyle background information on variables that may act as confounders; these include smoking habits, annual income, academic background, and level of physical activity both at baseline and at the end of the study.

A number of individual studies and several meta-analyses have reported favorable outcomes with omega-3 fatty acid intervention in patients with NAFLD [27,36,37]. Despite a high degree of heterogeneity in patient population, study duration, dose and form of omega-3 fatty acids, a recent meta-analysis concluded that omega- 3 fatty acids are associated with significant improvements in liver fat content and the liver enzymes ALT and GGT when taking approximately $3 \mathrm{~g} /$ day of EPA and DHA [27]. The positive effect of omega-3 fatty acids on liver fat was also confirmed in an earlier meta-analysis [37]. Surprisingly, only four of the eight trials performed to date included some form of measurement of EPA and DHA enrichment following intervention [38-41]. A strength of the current study is the measurement of both omega- 3 fatty acids and omega-6: omega-3 fatty acid ratio, as well as the quantification of individual EPA and DHA in RBCs at baseline and study completion. This has enabled additional regression analyses to be performed, which suggest an inverse relationship between change in absolute RBC EPA + DHA and change in liver fat content and liver enzyme concentrations, although these data are exploratory and not supported by statistical analysis. Similar findings were reported in a study of high dose omega-3 in NAFLD patients where beneficial effects on liver fat content correlated with DHA content in RBCs [40]. Another strength of the current study was the use of MRI-PDFF to accurately assess change in liver fat content, which is the most accurate assessment method besides highly invasive liver biopsy [42].

A limitation of this study was the finding of a relatively low level of hepatic steatosis in participants, which restricted the potential for more significant effects to be observed on liver-related outcomes.

Post hoc use of the FLI to stratify patients showed an association between higher FLI scores and greater decrease in hepatic fat in the omega- 3 intervention group. FLI scores below 30 are predictive of a liver without steatosis [10]. The high number of subjects with FLI $<30$ confirms that this study recruited a relatively healthy population. However, highly statistically significant improvements in hepatic fat content were seen in those with a baseline FLI $>40$, suggesting that this patient group can receive beneficial effects of intervention compared to placebo. Such a use of FLI is in accordance 
with the aims of its developers who propose that the "potential clinical uses of FLI include the selection of subjects to be referred for ultrasonography and the identification of (NAFLD) patients for intensified lifestyle counselling" [10]. Stratification by MRI-PDFF assessed steatosis levels was unable to identify omega-3 "responders"; however, the FLI score was able to do this. In this case, we can consider the study population as a population enriched for the presence of hepatic steatosis. The FLI score is composed, in part, of measurements of triglycerides and the liver enzyme GGT. Increased triglycerides in the liver is the cause of steatosis and increased liver enzymes are a consequence of liver damage. Plasma triglycerides are sensitive to omega-3 fatty acid intervention. In a meta-analysis, omega-3 fatty acids were shown to decrease liver enzymes (in particular GGT) in NAFLD patients, providing evidence that omega-3 intervention has a beneficial effect on liver cell physiology [27]. As such, the FLI may represent an easily available and economic set of biomarkers of relevance for identifying omega-3 sensitive patients and future studies may consider using this tool a priori for patient selection/stratification purposes.

\section{Conclusions}

The current randomized placebo-controlled study supports the use of omega-3 supplementation to increase the omega-3 index in NAFLD patients, significantly greater than that obtained by dietary recommendation alone. This study is therefore in line with recently published research which encourages NAFLD patients to increase their intakes of $n$-3 LC-PUFAs and confirms their beneficial effect [27]. The liver fat content of patients was significantly reduced amongst both placebo and intervention arms, thereby masking any effects omega- 3 may have had on the fat content of the liver. Limitations and suggestions for future study design are discussed. In a post-hoc analysis significant placebo adjusted reductions in liver fat were seen in sub-populations with a high FLI score. This economic and easily available test may provide a simple means of identifying omega-3 responsive patients.

Supplementary Materials: The following are available online at http://www.mdpi.com/2072-6643/10/8/1126/ s1.

Author Contributions: D.T., M.B.A., Y.Q. and P.C.C. designed the study; D.T., M.B.A. and Y.Q. administered and oversaw the study; D.T., M.B.A., Y.Q. and P.C.C. interpreted data; J.K.I. drafted the manuscript; D.T., Y.Q. and P.C.C. reviewed and edited the manuscript; all authors approved the final version of the manuscript.

Funding: This study was funded by BASF AS.

Conflicts of Interest: D.T. and Y.Q. are employees of BASF AS; M.B.A. is a former employee of BASF AS; P.C.C. is an advisor to BASF AS; J.K.I. has no conflicts to declare.

\section{References}

1. Chalasani, N.; Younossi, Z.; Lavine, J.E.; Diehl, A.M.; Brunt, E.M.; Cusi, K.; Charlton, M.; Sanyal, A.J. The diagnosis and management of non-alcoholic fatty liver disease: Practice Guideline by the American Association for the Study of Liver Diseases, American College of Gastroenterology, and the American Gastroenterological Association. Hepatology 2012, 55, 2005-2023. [CrossRef] [PubMed]

2. Levene, A.P.; Goldin, R.D. The epidemiology, pathogenesis and histopathology of fatty liver disease. Histopathology 2012, 61, 141-152. [CrossRef] [PubMed]

3. McCullough, A.J. The clinical features, diagnosis and natural history of nonalcoholic fatty liver disease. Clin. Liver Dis. 2004, 8, 521-533. [CrossRef] [PubMed]

4. Dowman, J.K.; Tomlinson, J.W.; Newsome, P.N. Pathogenesis of non-alcoholic fatty liver disease. Q. J. Med. 2010, 103, 71-83. [CrossRef] [PubMed]

5. Bedogni, G.; Miglioli, L.; Masutti, F.; Tiribelli, C.; Marchesini, G.; Bellentani, S. Prevalence of and risk factors for nonalcoholic fatty liver disease: The dionysos nutrition and liver study. Hepatology 2005, 42, 44-52. [CrossRef] [PubMed] 
6. $\quad$ LaBrecque, D.; Abbas, Z.; Anania, F.; Ferenci, P.; Khan, A.; Goh, K.; Hamid, S.; Isakov, V.; Lizarzabal, M.; Penaranda, M.; et al. World Gastroenterology Organisation Global Guidelines: Nonalcoholic fatty liver disease and nonalcoholic steatohepatitis. J. Clin. Gastroenterol. 2014, 48, 467-473. [CrossRef] [PubMed]

7. Neuschwander-Tetri, B.A.; Caldwell, S.H. Nonalcoholic steatohepatitis: Summary of an AASLD single topic conference. Hepatology 2003, 37, 1202-1219. [CrossRef] [PubMed]

8. Machado, M.; Marques-Vidal, P.; Cortez-Pinto, H. Hepatic histology in obese patients undergoing bariatric surgery. J. Hepatol. 2006, 45, 600-606. [CrossRef] [PubMed]

9. Targher, G.; Bertolini, L.; Padovani, R.; Rodella, S.; Tessari, R.; Zenari, L.; Day, C.; Arcaro, G. Prevalence of nonalcoholic fatty liver disease and its association with cardiovascular disease among Type 2 diabetic patients. Diabetes Care 2007, 30, 1212-1218. [CrossRef] [PubMed]

10. Bedogni, G.; Bellentani, S.; Miglioli, L.; Masutti, F.; Passalacqua, M.; Castiglione, A.; Tiribelli, C. The Fatty Liver Index: A simple and accurate predictor of hepatic steatosis in the general population. BMC Gastroenterol. 2006, 6, 1-7. [CrossRef] [PubMed]

11. Kotronen, A.; Yki-Järvinen, H. Fatty liver: A novel component of the metabolic syndrome. Arterioscler. Thromb. Vasc. Biol. 2008, 28, 27-38. [CrossRef] [PubMed]

12. Vuppalanchi, R.; Chalasani, N. Nonalcoholic fatty liver disease and nonalcoholic steatohepatitis: Selected practical issues in their evaluation and management. Hepatology 2009, 49, 306-317. [CrossRef] [PubMed]

13. Ekstedt, M.; Franzén, L.E.; Mathiesen, U.L.; Thorelius, L.; Holmqvist, M.; Bodemar, G.; Kechagias, S. Long-term follow-up of patients with NAFLD and elevated liver enzymes. Hepatology 2006, 44, 865-873. [CrossRef] [PubMed]

14. Chitturi, S.; Abeygunasekera, S.; Farrell, G. NASH and insulin resistance: Insulin hypersecretion and specific association with the insulin resistance syndrome. Hepatology 2002, 2, 373-379. [CrossRef] [PubMed]

15. Allard, J.P.; Aghdassi, E.; Mohammed, S.; Raman, M.; Avand, G.; Arendt, B.M.; Jalali, P.; Kandasamy, T.; Prayitno, N.; Sherman, M.; et al. Nutritional assessment and hepatic fatty acid composition in non-alcoholic fatty liver disease (NAFLD): A cross-sectional study. J. Hepatol. 2008, 48, 300-307. [CrossRef] [PubMed]

16. Araya, J.; Rodrigo, R.; Videla, L.; Thielemann, L.; Orellana, M.; Pettinelli, P.; Poniachik, J. Increase in long-chain polyunsaturated fatty acid $n-6 / n-3$ ratio in relation to hepatic steatosis in patients with non-alcoholic fatty liver disease. Clin. Sci. 2004, 106, 635-643. [CrossRef] [PubMed]

17. Pettinelli, P.; del Pozo, T.; Araya, J.; Rodrigo, R.; Araya, A.V.; Smok, G.; Csendes, A.; Gutierrez, L.; Rojas, J.; Korn, O.; et al. Enhancement in liver SREBP-1c/PPAR- $\alpha$ ratio and steatosis in obese patients: Correlations with insulin resistance and $n-3$ long-chain polyunsaturated fatty acid depletion. Biochim. Biophys. Acta-Mol. Basis. Dis. 2009, 1792, 1080-1086. [CrossRef] [PubMed]

18. Elizondo, A.; Araya, J.; Rodrigo, R.; Poniachik, J.; Csendes, A.; Maluenda, F.; Díaz, J.C.; Signorini, C.; Sgherri, C.; Comporti, M.; et al. A polyunsaturated fatty acid pattern in liver and erythrocyte phospholipids from obese patients. Obesity 2007, 15, 24-31. [CrossRef] [PubMed]

19. Rose, M.; Veysey, M.; Lucock, M.; Niblett, S.; King, K.; Baines, S.; Garg, M.L. Association between erythrocyte omega-3 polyunsaturated fatty acid levels and fatty liver index in older people is sex dependent. J. Nutr. Intermed. Metab. 2016, 5, 78-85. [CrossRef]

20. Toshimitsu, K.; Matsuura, B.; Ohkubo, I.; Niiya, T.; Furukawa, S.; Hiasa, Y.; Kawamura, M.; Ebihara, K.; Onji, M. Dietary habits and nutrient intake in non-alcoholic steatohepatitis. Nutrition 2007, 23, 46-52. [CrossRef] [PubMed]

21. Cortez-Pinto, H.; Jesus, L.; Barros, H.; Lopes, C.; Moura, M.C.; Camilo, M.E. How different is the dietary pattern in non-alcoholic steatohepatitis patients? Clin. Nutr. 2006, 25, 816-823. [CrossRef] [PubMed]

22. Clarke, S.D. Molecular mechanism for polyunsaturated fatty acid regulation of gene transcription. Am. J. Physiol. 2001, 281, 865-869. [CrossRef] [PubMed]

23. Bouzianas, D.G.; Bouziana, S.D.; Hatzitolios, A.I. Potential treatment of human nonalcoholic fatty liver disease with long-chain omega-3 polyunsaturated fatty acids. Nutr. Rev. 2013, 71, 753-771. [CrossRef] [PubMed]

24. Hodson, L.; Bhatia, L.; Scorletti, E.; Smith, D.E.; Jackson, N.C.; Shojaee-Moradie, F.; Umpleby, M.; Calder, P.C.; Byrne, C.D. Docosahexaenoic acid enrichment in NAFLD is associated with improvements in hepatic metabolism and hepatic insulin sensitivity: A pilot study. Eur. J. Clin. Nutr. 2017, 71, 973-979. [CrossRef] [PubMed] 
25. Chen, L.; Wang, Y.; Xu, Q.; Chen, S.-S. Omega-3 fatty acids as a treatment for non-alcoholic fatty liver disease in children: A systematic review and meta-analysis of randomized controlled trials. Clin. Nutr. 2018, 37, 516-521. [CrossRef] [PubMed]

26. De Castro, G.S.; Calder, P.C. Non-alcoholic fatty liver disease and its treatment with n-3 polyunsaturated fatty acids. Clin. Nutr. 2017. [CrossRef] [PubMed]

27. Musa-Veloso, K.; Venditti, C.; Lee, H.; Darch, M.; Floyd, S.; West, S.; Simon, R. Systematic review and meta-analysis of controlled intervention studies on the effectiveness of long chain omega-3 fatty acids in patients with non-alcoholic fatty liver disease. Nutr. Rev. 2018, 76, 581-602. [CrossRef] [PubMed]

28. Katan, M.B.; Deslypere, J.P.; van Birgelen, A.P.; Penders, M.; Zegwaard, M. Kinetics of the incorporation of dietary fatty acids into serum cholesteryl esters, erythrocyte membranes, and adipose tissue: An 18-month controlled study. J. Lipid Res. 1997, 38, 2012-2022. [PubMed]

29. Krauss, R.; Eckel, R.; Howard, B.; Appel, L.J.; Daniels, S.R.; Deckelbaum, R.J.; Erdman, J.W.; Kris-etherton, P.; Goldberg, I.J.; Kotchen, T.A.; et al. AHA dietary guidelines. Circulation 2000, 102, 2284-2299. [CrossRef] [PubMed]

30. Jørgensen, M.; Ott, P.; Michaelsen, K.; Porsgaard, T.; Jensen, F.; Lanng, S. Long-chain PUFA in granulocytes, mononuclear cells, and RBC in patients with cystic fibrosis: Relation to liver disease. J. Pediatr. Gastroenterol. Nutr. 2012, 55, 76-81. [CrossRef] [PubMed]

31. Petit, J.M.; Guiu, B.; Duvillard, L.; Jooste, V.; Brindisi, M.C.; Athias, A.; Bouillet, B.; Habchi, M.; Cottet, V.; Gambert, P.; et al. Increased erythrocytes n-3 and n-6 polyunsaturated fatty acids is significantly associated with a lower prevalence of steatosis in patients with type 2 diabetes. Clin. Nutr. 2012, 31, 520-525. [CrossRef] [PubMed]

32. Arterburn, L.M.; Hall, E.B.; Oken, H. Distribution, interconversion, and dose response of n-3 fatty acids in humans. Am. J. Clin. Nutr. 2006, 83, 1467S-1476S. [CrossRef] [PubMed]

33. Tobin, D. BASF AS, Norway. CONDIN study. Unpublished work. 2018.

34. Browning, L.M.; Walker, C.G.; Mander, A.P.; West, A.L.; Madden, J.; Gambell, J.M.; Young, S.; Wang, L.; Jebb, S.A.; Calder, P.C. Incorporation of eicosapentaenoic and docosahexaenoic acids into lipid pools when given as supplements providing doses equivalent to typical intakes of oily fish. Am. J. Clin. Nutr. 2012, 96, 748-758. [CrossRef] [PubMed]

35. Chalasani, N.; Vuppalanchi, R.; Rinella, M.; Middleton, M.S.; Siddiqui, M.S.; Barritt, A.S.; Kolterman, O.; Flores, O.; Alonso, C.; Iruarrizaga-Lejarreta, M.; et al. Randomised clinical trial: A leucine-metformin-sildenafil combination (NS-0200) vs placebo in patients with non-alcoholic fatty liver disease. Aliment. Pharmacol. Ther. 2018, 47, 1639-1651. [CrossRef] [PubMed]

36. He, X.-X.; Wu, X.-L.; Chen, R.-P.; Chen, C.; Liu, X.-G.; Wu, B.-J.; Huang, Z.-M. Effectiveness of omega-3 polyunsaturated fatty acids in non-alcoholic fatty liver disease: A meta-analysis of randomized controlled trials. PLoS One 2016, 11, e0162368. [CrossRef] [PubMed]

37. Parker, H.M.; Johnson, N.A.; Burdon, C.A.; Cohn, J.S.; Connor, H.T.O.; George, J. Omega-3 supplementation and non-alcoholic fatty liver disease: A systematic review and meta-analysis. J. Hepatol. 2012, 56, 944-951. [CrossRef] [PubMed]

38. Capanni, M.; Calella, F.; Biagini, M.R.; Genise, S.; Raimondi, L.; Bedogni, G.; Svegliati-Baroni, G.; Sofi, F.; Milani, S.; Abbate, R.; et al. Prolonged n-3 polyunsaturated fatty acid supplementation ameliorates hepatic steatosis in patients with non-alcoholic fatty liver disease: A. pilot study. Aliment. Pharmacol. Ther. 2006, 23, 1143-1151. [CrossRef] [PubMed]

39. Qin, Y.; Zhou, Y.; Chen, S.H.; Zhao, X.L.; Ran, L.; Zeng, X.L.; Wu, Y.; Chen, J.L.; Kang, C.; Shu, F.R.; et al. Fish oil supplements lower serum lipids and glucose in correlation with a reduction in plasma fibroblast growth factor 21 and prostaglandin E2 in nonalcoholic fatty liver disease associated with hyperlipidemia: A randomized clinical trial. PLoS ONE 2015, 10, 1-13. [CrossRef] [PubMed]

40. Scorletti, E.; Bhatia, L.; Mccormick, K.G.; Clough, G.F.; Nash, K.; Hodson, L.; Moyses, H.E.; Calder, P.C.; Byrne, C.D. Effects of purified eicosapentaenoic and docosahexaenoic acids in nonalcoholic fatty liver disease: Results from the WELCOME study. Hepatology 2014, 60, 1211-1221. [CrossRef] [PubMed] 
41. Vega, G.; Chandalia, M.; Szczepaniak, L.; Grundy, S. Effects of N-3 fatty acids on hepatic triglyceride content in humans. J. Investig. Med. 2008, 56, 780-785. [CrossRef] [PubMed]

42. Dulai, P.; Sirlin, C.; Loomba, R. MRI and MRE for non-invasive quantitative assessment of hepatic steatosis and fibrosis in NAFLD and NASH: Clinical trials to clinical practice. J. Hepatol. 2016, 65, 1006-1016. [CrossRef] [PubMed]

(C) $(1)$ BY
(C) 2018 by the authors. Licensee MDPI, Basel, Switzerland. This article is an open access article distributed under the terms and conditions of the Creative Commons Attribution (CC BY) license (http:/ / creativecommons.org/licenses/by/4.0/). 


\title{
Review \\ Muscle Loss in Chronic Liver Diseases: The Example of Nonalcoholic Liver Disease
}

\author{
Jean-Pascal De Bandt * (D), Prasanthi Jegatheesan and Naouel Tennoune-El-Hafaia \\ EA4466 PRETRAM, Faculté de Pharmacie de Paris, USPC, 75006 Paris, France; \\ pira_jegatheesan@hotmail.com (P.J.); naouel.el-hafaia@parisdescartes.fr (N.T.-E.-H.) \\ * Correspondence: jean-pascal.de-bandt@parisdescartes.fr; Tel.: +33-170-649-441
}

Received: 6 August 2018; Accepted: 23 August 2018; Published: 1 September 2018

\begin{abstract}
Recent publications highlight a frequent loss of muscle mass in chronic liver diseases, including nonalcoholic fatty liver disease (NAFLD), and its association with a poorer prognosis. In NAFLD, given the role of muscle in energy metabolism, muscle loss promotes disease progression. However, liver damage may be directly responsible of this muscle loss. Indeed, muscle homeostasis depends on the balance between peripheral availability and action of anabolic effectors and catabolic signals. Moreover, insulin resistance of protein metabolism only partially explains muscle loss during NAFLD. Interestingly, some data indicate specific alterations in the liver-muscle axis, particularly in situations such as excess fructose/sucrose consumption, associated with increased hepatic de novo lipogenesis (DNL) and endoplasmic reticulum stress. In this context, the liver will be responsible for a decrease in the peripheral availability of anabolic factors such as hormones and amino acids, and for the production of catabolic effectors such as various hepatokines, methylglyoxal, and uric acid. A better understanding of these liver-muscle interactions could open new therapeutic opportunities for the management of NAFLD patients.
\end{abstract}

Keywords: sarcopenia; protein metabolism; insulin resistance; endoplasmic reticulum stress; hepatokine; amino acid; uric acid; methylglyoxal

\section{Introduction}

The loss in muscle mass, often wrongly referred to as sarcopenia, has long been largely neglected in liver diseases. This is probably partly because of possible difficulties in its assessment due, for example, to fluid retention. However, several recent studies have shown frequent loss of muscle mass associated with poor prognosis in various chronic liver diseases. The progressive deterioration of muscle trophicity in these diseases is therefore receiving increasing attention. Overall, studies demonstrate muscle loss in nearly $60 \%$ of patients with end-stage liver diseases and this is associated with a worse prognosis [1]. However, muscle loss is already present in the early stages of liver disease and worsens with its severity [2]. This is particularly the case of nonalcoholic fatty liver disease (NAFLD), which encompasses a broad spectrum of disorders ranging from simple steatosis to nonalcoholic steatohepatitis (NASH), cirrhosis, and hepatocellular carcinoma, and where loss of muscle mass can occur very early during the disease. Indeed, in addition to the disturbances in muscle homeostasis related to metabolic disorders, several pieces of evidence indicate a specific role of the alterations in liver function in muscle loss. This is particularly important as the progressive deterioration of muscle trophicity promotes NAFLD progression, given the role of the muscle in energy metabolism. The alterations in the liver-muscle axis, due to hepatic steatosis, initiate a vicious circle in which liver disease favors defective muscle protein accretion and in which muscle loss favors metabolic alterations as well as hepatic steatosis and inflammation.

After examining some epidemiological data on muscle loss in chronic liver diseases and NAFLD, the authors of this review focus on muscle homeostasis and the different mechanisms by which NAFLD 
can act on muscle protein metabolism, including the decreased peripheral availability and action of anabolic factors such as hormones and amino acids, and the production of catabolic effectors such as various hepatokines, methylglyoxal, and uric acid.

It should be noted that many authors speak of sarcopenia while patients present only this loss of muscle mass. Indeed, the European society for clinical nutrition and metabolism defines sarcopenia, a term first coined for geriatric patients, as "a syndrome of its own characterized by the progressive and generalized loss of skeletal muscle mass, strength and function (performance) with a consequent risk of adverse outcomes" [3].

\section{Muscle Loss in Chronic Liver Diseases}

The interest in muscle mass loss in liver diseases is the result of recent evidence of its frequency and its consequences in terms of morbidity and mortality. A meta-analysis of 7 Asian studies and 13 Western studies in patients with liver cirrhosis of various etiologies concluded that muscle loss was present in $48.1 \%$ of patients, more prevalent in men $(61.6 \%)$ than in women (36\%) [4]. Several studies have shown that muscle loss in liver transplant candidates is associated with increased mortality [2] and increased risk of complications [4] In a recent systematic review and meta-analysis, the pooled hazard ratios of muscle loss was 1.84 (95\% CI: 1.11-3.05, $p=0.02)$ for post-transplantation mortality [5]. Moreover, muscle loss appeared to be associated with increased complications, such as infections [6], ascites, encephalopathy, and variceal hemorrhage [7].

More specifically for NAFLD, most data come from studies in the Korean population. Using data from the Korean National Health and Nutrition Examination Surveys (KNHANES), Lee et al. [8] showed that NAFLD was present in 2761 (28.5\%) out of 9676 subjects, and that 337 NAFLD patients $(12.2 \%)$ had low muscle mass. Hong et al. [9] studied the relationship between muscle mass and liver disease evaluated by serum gamma-glutamyl transferase (GGT) in 3193 adults aged over 50 years from the fifth KNHANES. They observed that patients in the highest GGT quintile were 2.3 times more likely to have low muscle mass than those in the lowest quintile. In a seven-year Korean longitudinal study [10] on 12,624 subjects without initial NAFLD, followed for occupational medicine purpose, $14.8 \%$ of the subjects developed NAFLD and the highest tertile of muscle mass was inversely associated with the incidence of NAFLD compared to the lowest tertile. Finally, in the study by Koo et al. [11] of 309 subjects with signs of hepatic steatosis, the prevalence of low muscle mass was $8.7 \%$ in patients without NAFLD and $17.9 \%$ and 35\%, respectively, in biopsy-proven nonalcoholic fatty liver and NASH patients. In this study, as in Lee's study [8], low muscle mass was associated with fibrosis (OR: $2.05,95 \%$ CI: 1.01-4.16, $p=0.034$ ), independently of body mass index and insulin resistance. This relationship between low muscle mass and fibrosis has also been confirmed in a European cohort of biopsy-proven NAFLD patients [12].

Given the importance of muscle in energy and nitrogen homeostasis, this muscle loss negatively affects whole-body metabolism [13]. The consequences on hepatic metabolism are clearly shown, for example, in the study by Flannery et al. [14] of healthy sedentary elderly subjects. These authors observed, after a test meal, a twofold higher hepatic de novo lipogenesis (DNL), a threefold higher postprandial hepatic triglyceride (TG) content, and significantly increased plasma TG compared to healthy, young subjects. Note that, in a study of 452 apparently healthy adults from the Korean Sarcopenic Obesity Study [15], patients in the lowest quartile of muscle mass had a 5.2-fold increased risk of NAFLD compared to the highest quartile (95\% CI: $1.63-16.33, p=0.041)$. In this study, hepatic steatosis and skeletal muscle mass index were negatively correlated with insulin resistance, low grade inflammation, and arterial stiffness, and positively correlated with plasma TG and alanine aminotransferase.

\section{Muscle Protein Homeostasis}

To understand the mechanisms by which hepatic disorders may affect muscle protein homeostasis, the authors will first briefly review some main aspects of the control of protein metabolism in muscle. 
Muscle homeostasis in healthy individuals depends on the fasting-feeding alternation and, at the cell level, on the balance between protein synthesis and catabolism. Protein synthesis is activated by anabolic factors such as hormones (insulin and insulin-like growth factor 1 [IGF-1]), and amino acid (AAs) availability (with AAs such as leucine and arginine playing specific regulatory roles), and it is inhibited by nutrient deficiency and inflammatory processes. Conversely, protein catabolism is activated by energy and AA deficiency and inflammatory processes, and it is inhibited by anabolic hormones [16].

During the postprandial period, anabolic effects of feeding result from increased protein synthesis and decreased catabolism due to increased availability and action of anabolic effectors. AAs stimulate protein synthesis; insulin has a permissive effect and increases the supply of nutrients to muscles through its vasodilatory properties. Insulin-induced inhibition of proteolysis is enhanced by AA availability [17]. In addition, the activation of protein synthesis depends on specific AAs such as leucine and arginine; thus, the anabolic effects of feeding also depend on specific qualitative variations in AA availability [18]. Finally, the vasodilatory effects of insulin play an important role in the physiological coupling between hemodynamic and metabolic homeostasis [19]. The increase in the metabolic activity of skeletal muscle requires adequate availability of the substrate for nitric oxide $\left({ }^{\circ} \mathrm{NO}\right)$ synthesis (i.e., arginine) to increase blood flow for substrate supply.

On the long term, maintaining skeletal muscle mass also depends on the growth hormone (GH)/IGF-1 axis. Blood IGF-I is $80 \%$ dependent on its GH-induced liver production. Plasma IGF-I is associated positively with lean body mass and muscle function, and negatively with body fat mass. IGF-I action is also regulated by its binding to circulating IGF-1 binding proteins (IGFBP) produced by the liver [20].

Overall, the maintenance of muscle mass requires that the muscles be responsive to these anabolic effectors and that the anabolic effectors actually and quantitatively reach the muscles. As indicated below, NAFLD may be associated with a decrease in muscle mass as a consequence of (i) insulin-resistance; (ii) decreased IGF-1 production by the liver; (iii) decreased AA flows to the muscles due to increased splanchnic utilization; (iv) a defective postprandial peripheral vasodilatory response; or (v) the catabolic effect of various mediators, including hepatokines, produced by the liver in steatosis-induced situations of endoplasmic reticulum (ER) stress.

\section{Insulin Resistance and Muscle Homeostasis}

A first process that can promote muscle loss is insulin resistance. Depending on the pathophysiological situation, insulin resistance may either precede NAFLD, due to increased adiposity, or result from NAFLD as the steatotic liver is the site of increased glucose and TG production; in all cases, the two processes reinforce each other. Decreased sensitivity of protein turnover to insulin action has been described in both type 1 and type 2 diabetes [21]. This decrease in insulin sensitivity may result from several mechanisms such as lipotoxicity, glucotoxicity, and inflammation.

Increased circulating free fatty acid (FFA) concentration favors ectopic lipid deposition and altered insulin signaling due to the accumulation of TG deposition, for example, in muscle [22]. Moreover, specific FFAs (e.g., palmitate) and lipid metabolites (e.g., ceramide and diacylglycerol) may induce insulin resistance. It should be noted that palmitate also activates toll-like receptor 4 (TLR4) and triggers inflammatory processes [23].

Excess glucose induces oxidative stress in muscles and the production of advanced glycation end products (AGEs) that interact with the receptors of AGEs on muscle cells. These receptors induce the production of reactive oxygen species and cause inflammation via mitogen-activated protein kinases and nuclear factor $\mathrm{kB}$ pathways [24]. For example, Howard et al. [25] showed that high glucose and AGEs induce a defect in myocyte membrane repair.

Excess adipose tissue, particularly visceral adipose tissue, is associated with the increased secretion of pro-inflammatory cytokines, such as tumor necrosis factor $\alpha$ and interleukin-6, and adipokines (leptin and resistin, whereas insulin-sensitizing adiponectin production decreases) 
which promote insulin resistance [26]. Interestingly, in the authors' model of fructose-induced NAFLD in rats, muscle loss was associated with increased visceral adiposity and an inflammatory state [27].

A last mechanism by which insulin resistance may contribute to muscle loss is the above-mentioned defect in the postprandial peripheral vasodilatory response as it reduces the flow of anabolic factors to the muscle. Indeed, in addition to its essential metabolic actions, insulin stimulates the production of nitric oxide $\left({ }^{\circ} \mathrm{NO}\right)$ by endothelial ${ }^{\circ} \mathrm{NO}$ synthase (eNOS). Therefore, in the post-prandial period, insulin induces vasodilation, increased blood flow and increased availability of substrates and hormones for target tissues. Insulin resistance is associated with impaired endothelium-dependent vasodilation and vascular function [19]. There is thus a reciprocal interaction between insulin resistance and the defect in endothelial ${ }^{\circ} \mathrm{NO}$ production. The pathophysiological mechanisms linking these two processes contribute to metabolic disorders and the cardiovascular features of the metabolic syndrome. Moreover, insulin resistance states are associated with decreased availability of an important AA, arginine, the substrate of eNOS for ${ }^{\circ} \mathrm{NO}$ synthesis.

\section{Metabolic Disorders, Liver Steatosis, and Endoplasmic Reticulum Stress}

Although insulin resistance may affect muscle function, it cannot by itself explain the defect in muscle protein accretion associated with NAFLD and, more specifically, with the excessive consumption of fructose. Therefore, another process must contribute to muscle protein loss. One explanation could be excess DNL, the cause of steatosis, leading to hepatic oxidative stress, inflammation, and ER stress.

Under normal conditions, at the hepatic level, FFAs come from white adipose tissue (WAT) lipolysis during the fasting period, from diet, and from DNL. FFAs will either be esterified into TG and then exported to the blood as very-low-density lipoproteins (VLDL) or degraded by $\beta$-oxidation. Pathological accumulation of lipids in the liver may result from the excessive entry of FFAs released by insulin-resistant WAT, particularly visceral adipose tissue, excessive activation of DNL, and alterations in $\beta$-oxidation and lipid excretion as VLDL. In NAFLD patients, approximately $60 \%$ of FFAs come from lipolysis in WAT, nearly 25\% result from DNL, and the remainder come from diet [28]. Nutritional factors, such as a diet rich in sucrose/fructose and lipids, and the disequilibrium of the energy balance thus play a major role in the occurrence of this pathology.

Excessive sucrose or fructose consumption can significantly contribute to liver steatosis and disease progression. Experimental studies repeatedly demonstrated that a high-sucrose or high-fructose diet promotes subcutaneous and visceral obesity, insulin resistance, dyslipidemia, increased blood uric acid, and hypertension [29]. Even moderate doses of fructose can induce metabolic syndrome, fatty liver, and type 2 diabetes even in the absence of excess energy intake [30]. In humans, a high-fructose intake ( $>1.5 \mathrm{~g} / \mathrm{kg} /$ day) can double the intrahepatic fat content within six days [31]. Conversely, in a pilot study of 15 NAFLD patients, Volynets et al. [32] showed that a dietary intervention focusing on a 50\% reduction in fructose intake was associated with decreased hepatic steatosis and improved liver function and glucose tolerance. This can be at least in part explained by the specificities of fructose metabolism in the liver and the excessive activation of DNL. Indeed, fructose is both a substrate and an activator of DNL through the activation of carbohydrate-responsive element-binding protein (ChREBP) and sterol regulatory element-binding protein 1c, two transcription factors controlling the main enzymes involved in DNL [33]. Excess DNL causes oxidative stress in liver cells and is associated with the development of hepatic inflammation and insulin resistance [34,35]. It also induces ER stress, which contributes to the aggravation of NAFLD [34]. The superposition of ER stress and inflammation may lead to the production of various mediators, such as cytokines, hepatokines, and carbohydrate and lipid derivatives, which can act at the whole-body level and contribute to alterations in whole-body metabolism [36,37].

With respect to muscle mass loss, studies $[27,38,39]$ have shown that excessive consumption of fructose or sucrose leads to changes in body composition with fat accumulation and alterations of muscle protein pool. Interestingly, in a study of community-dwelling elderly subjects, Laclaustra et al. [40] showed an association between added sugar consumption and the appearance of frailty. Several lines 
of evidence indicate that sucrose-related alterations in liver metabolism, at least in part due to the conversion of sucrose into lipids in the process of DNL, lead to changes in peripheral organ metabolism and muscle protein accretion. This is illustrated by the comparison of mouse models of primary steatosis, resulting from an increase in hepatic DNL, and secondary steatosis resulting from ectopic lipid deposition, primary steatosis being associated with significantly lower lean body mass [41].

\section{Hepatic Endoplasmic Reticulum Stress and Muscle Homeostasis}

The question therefore arises about the mechanisms by which these alterations in liver homeostasis may affect muscle protein metabolism.

\subsection{Alterations of the GH/IGF-1 Axis}

The bidirectional relationship between steatosis and insulin resistance has already been mentioned. Steatosis is also associated with alterations of the GH/IGF-1 axis. While data on plasma GH are inconsistent [42,43], studies agree on a decrease in plasma IGF-1 in NAFLD [42-44]. The liver being the main organ contributing to plasma IGF-1 concentration, this suggests that NAFLD alters IGF-1 production. Runchey et al. [45] showed in 4172 adults who participated in the NHANES III that the highest quartiles of IGF-1 and IGF-1/IGFBP-3 were associated with a lower likelihood and grade of NAFLD. Note that in an exploratory study of healthy volunteers on a high-sucrose diet, the authors observed an association between increased liver lipid content and decreased plasma IGF-1 [46]. In a model of Western diet-induced NAFLD in mice, steatosis was associated with a $40 \%$ decrease in IGF-1 hepatic expression [47]. This seems to be specific for liver steatosis, as suggested by Chishima et al. [43] in a study of Japanese patients with NAFLD or Hepatitis C virus (HCV) chronic liver disease where IGF-1 levels were decreased in patients with NAFLD but not with HCV. Moreover, serum IGFBP-3 levels were also decreased, indicating a reduction in blood half-life of IGF-1. Conversely, hepatic ER stress is associated with the stimulation of IGFBP1 secretion [48]. IGFBP1 is a modulator of IGF-1 action and its overexpression is associated with hyperinsulinemia and glucose intolerance [49]. Experimentally, hepatic steatosis-related alterations in GH/IGF-1 axis are associated with a decrease in muscle myofibrillar protein content and a reduction in muscle strength in the absence of significant inflammation [47].

\subsection{Alterations of Amino Acid Interorgan Fluxes}

Disorders of nitrogen homeostasis in situations of stimulated DNL may be an early event during the development of steatosis [33]. In an experimental model of fructose-induced NAFLD in rats, the prolonged administration of a fructose-rich diet was associated with a decrease in lean body mass, an increase in visceral fat mass, and changes in AA plasma levels, notably in arginine bioavailability. Conversely, an increase in AA availability enable to decrease DNL and associated alterations in body composition [27]. In healthy volunteers, an essential AA supplement reduced fructose-induced intrahepatic lipid accumulation [50]. Interestingly, based on their results showing alterations in blood AA profile in non-diabetic NAFLD patients with or without obesity, Gaggini et al. [51] concluded that the observed increase in AAs such as branched-chain and aromatic AAs resulted from an increase in muscle proteolysis. Finally, in overweight hypertriglyceridemic patients, fructose infusion was associated with altered AA plasma levels and increased splanchnic extraction [33]. Similarly, postprandial AA availability was impaired in healthy volunteers receiving a high-sucrose diet [46]. Taken together, these data suggest that excess hepatic DNL is associated with a reorientation of AA fluxes towards the liver at the expense of muscle protein homeostasis.

\subsection{Hepatokines and Muscle Homeostasis}

In situations of hepatic ER stress, induced for example by steatosis, an increased production of some hepatokines and inflammatory cytokines has been demonstrated [52]. In cultured hepatocytes, steatosis has been associated with changes in the secretion of approximately 30 hepatokines [53]. 
Some of these mediators may be involved in muscle loss through a direct effect or through the deterioration of insulin sensitivity. For example:

(1) Fetuin A is a glycoprotein associated with alterations in glucose and lipid metabolism. Both experimental and clinical studies showed increased hepatic expression and plasma levels of Fetuin A during NAFLD. Studies in humans showed a close relationship between plasma Fetuin A and the metabolic syndrome [54]. Its production by hepatocytes is strongly stimulated by ER stress [55]. This protein produced primarily by the liver is an endogenous inhibitor of the insulin receptor tyrosine kinase in skeletal muscle [56]. Fetuin A may also bind TLR4, stimulating inflammatory pathways [52].

(2) Fibroblast growth factor 21 (FGF21) is a mediator produced primarily by the liver that contributes to the regulation of energy metabolism and insulin sensitivity [57]. FGF21 is now recognized as a key player in the adaptive response to starvation and feeding [58]. Fructose induces FGF21 production by the activation of the transcription factor ChREBP [59]. ER stress modulates FGF21 expression in the liver [60]. FGF21 is an intriguing hepatokine as it is generally considered as beneficial [59]; however, some data indicate either states of FGF21 resistance or deleterious effects, as suggested by the very high FGF21 plasma levels observed in patients with insulin resistance [52]. In male and female rats fed a high-fat high-fructose diet, FGF21 was increased only in males and this was associated with marked liver damage, inflammation, and oxidative stress [61]. AA deprivation is also a potent inducer of hepatic FGF21 production via ER stress response [62]; very high levels of FGF21 could potentially alter nitrogen homeostasis in the context of NAFLD-associated muscle mass loss. It should be noted that transgenic mice overexpressing FGF21 exhibit increased gluconeogenesis in the fed state, and that acute treatment with FGF21 induces key enzymes in the gluconeogenic pathway [62], suggesting an increased hepatic utilization of AAs.

(3) Hepassocin (HPS, hepatocyte-derived fibrinogen-related protein 1 [HFREP1], fibrinogen-like protein 1 [FGL1]) is increased in NAFLD and induces insulin resistance in muscle [52]. Wu et al. have shown in humans that increased plasma HPS is independently associated with insulin resistance [63]. In primary hepatocytes or in vivo in mice, HPS expression is induced by ER stress. In differentiated myotubes, depending on the dose, HPS activated the c-Jun N-terminal kinase inflammatory pathway and altered insulin sensitivity [64]. In mice, hepatic HPS overexpression induced insulin resistance, while its knockdown was associated with improved insulin sensitivity in high-fat fed mice [63].

\subsection{Hepatic Production of Catabolic Factors}

Finally, carbohydrate metabolism may lead to the release by the liver of metabolites with significant peripheral effects.

During normal glycolysis, small amounts of methylglyoxal (MG) may be released. MG is produced by the fragmentation of the two products of aldolase B, namely glyceraldehyde-3-phosphate and dihydroxyacetone phosphate. MG is a powerful glycating agent leading to the generation of AGEs. Under normal conditions, its formation rate represents $0.1-0.4 \%$ of glycolytic flux [65], but the acceleration of glycolytic flux by fructose greatly increases MG formation. MG induces endothelial dysfunction [66]. Dhar et al. [67] showed an increase in MG formation associated with hypertension in fructose-fed rats. By acting on vascular function, MG may therefore contribute to a decrease in the peripheral availability of anabolic hormones and AAs.

A high-fructose intake results in an increased plasma uric acid level $[39,68]$. First, uric acid can contribute to fructose-induced metabolic disorders by impairing endothelial function, thereby decreasing insulin sensitivity by preventing insulin-induced muscle vasodilation. In addition, Zhu et al. [69] recently showed in mice that uric acid inhibits insulin signaling in muscle and induces insulin resistance. This effect may be related to uric acid-induced oxidative stress. In a study of severely obese subjects, Fabbrini et al. [70] observed a $40 \%$ decrease in insulin sensitivity in those with increased plasma uric 
acid. In an analysis of data from 7544 subjects who participated in the NHANES III, Beavers et al. [71] showed an association between increased blood uric acid levels and low muscle mass.

\section{Conclusions}

Chronic liver diseases contribute to alterations in muscle protein homeostasis, already at the stage of hepatic steatosis. While this review is limited to alterations in the liver-muscle axis, the situation is probably even more complex because NAFLD can be considered as a systemic disease affecting not only the liver and muscles, but also the gut and adipose tissue. High-fat and high-fructose diets, which promote the development of NAFLD, are associated with alterations in gut microbiota, increased gut permeability, and bacterial toxin translocation that can affect muscle homeostasis through systemic inflammation and insulin resistance [72]. Similarly, excessive sucrose consumption is associated with an increase in visceral adipose tissue in which has a higher production of pro-inflammatory factors and metabolic alterations than subcutaneous adipose tissue [26]. Muscle mass and function can be influenced by this adipose tissue dysfunction. The difficulty now becomes to establish the respective contribution of these different mechanisms in order to better define therapeutic targets.

Author Contributions: The three authors wrote the manuscript and approved the final content.

Funding: This research received no external funding.

Acknowledgments: None.

Conflicts of Interest: The authors declare no conflict of interest.

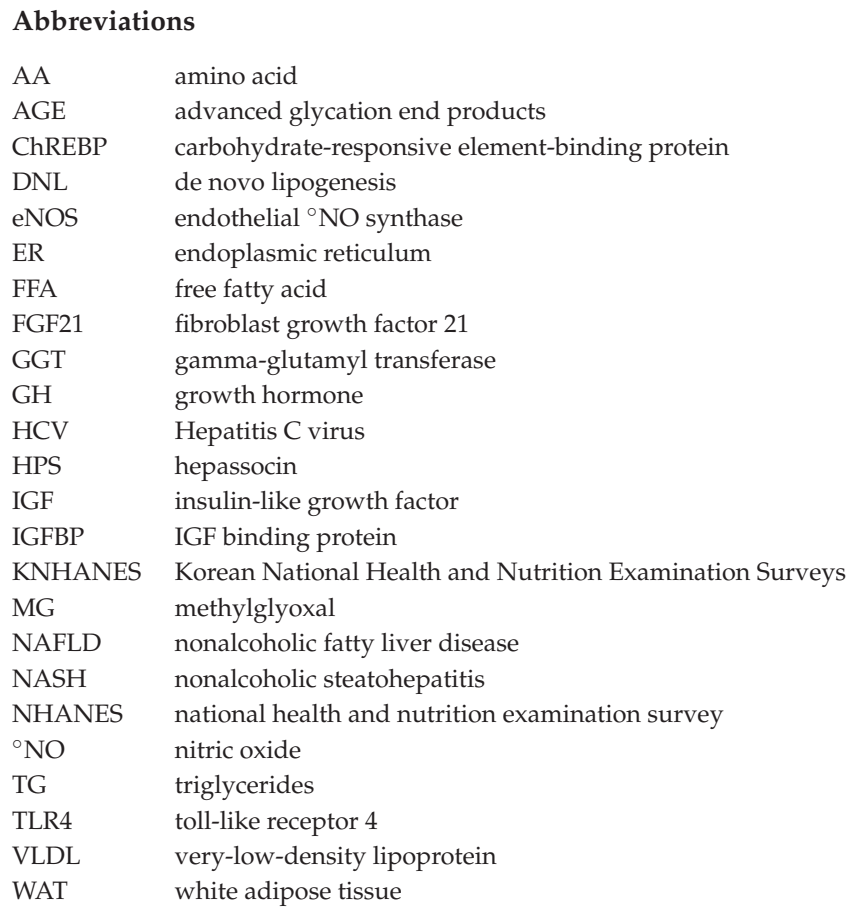

\section{References}

1. Bhanji, R.A.; Narayanan, P.; Allen, A.M.; Malhi, H.; Watt, K.D. Sarcopenia in hiding: The risk and consequence of underestimating muscle dysfunction in nonalcoholic steatohepatitis. Hepatol. Baltim. Md. 2017, 66, 2055-2065. [CrossRef] [PubMed] 
2. Anand, A.C. Nutrition and muscle in cirrhosis. J. Clin. Exp. Hepatol. 2017, 7, 340-357. [CrossRef] [PubMed]

3. Cederholm, T.; Barazzoni, R.; Austin, P.; Ballmer, P.; Biolo, G.; Bischoff, S.C.; Compher, C.; Correia, I.; Higashiguchi, T.; Holst, M.; et al. ESPEN guidelines on definitions and terminology of clinical nutrition. Clin. Nutr. 2017, 36, 49-64. [CrossRef] [PubMed]

4. Kim, G.; Kang, S.H.; Kim, M.Y.; Baik, S.K. Prognostic value of sarcopenia in patients with liver cirrhosis: A systematic review and meta-analysis. PLoS ONE 2017, 12, e0186990. [CrossRef] [PubMed]

5. Van Vugt, J.L.A.; Levolger, S.; de Bruin, R.W.F.; van Rosmalen, J.; Metselaar, H.J.; IJzermans, J.N.M. Systematic review and meta-analysis of the impact of computed tomography-assessed skeletal muscle mass on outcome in patients awaiting or undergoing liver transplantation. Am. J. Transplant. 2016, 16, 2277-2292. [CrossRef] [PubMed]

6. Lucidi, C.; Lattanzi, B.; Di Gregorio, V.; Incicco, S.; D’Ambrosio, D.; Venditti, M.; Riggio, O.; Merli, M. A low muscle mass increases mortality in compensated cirrhotic patients with sepsis. Liver Int. 2018, 38, 851-857. [CrossRef] [PubMed]

7. Montano-Loza, A.J.; Duarte-Rojo, A.; Meza-Junco, J.; Baracos, V.E.; Sawyer, M.B.; Pang, J.X.Q.; Beaumont, C.; Esfandiari, N.; Myers, R.P. Inclusion of sarcopenia within MELD (MELD-Sarcopenia) and the prediction of mortality in patients with cirrhosis. Clin. Transl. Gastroenterol. 2015, 6, e102. [CrossRef] [PubMed]

8. Lee, Y.; Kim, S.U.; Song, K.; Park, J.Y.; Kim, D.Y.; Ahn, S.H.; Lee, B.W.; Kang, E.S.; Cha, B.S.; Han, K.H. Sarcopenia is associated with significant liver fibrosis independently of obesity and insulin resistance in nonalcoholic fatty liver disease: Nationwide surveys (KNHANES 2008-2011). Hepatology 2016, 63, 776-786. [CrossRef] [PubMed]

9. Hong, N.; Lee, E.Y.; Kim, C.O. Gamma-glutamyl transferase is associated with sarcopenia and sarcopenic obesity in community-dwelling older adults: Results from the Fifth Korea National Health and Nutrition Examination Survey, 2010-2011. Endocr. J. 2015, 62, 585-592. [CrossRef] [PubMed]

10. Kim, G.; Lee, S.E.; Lee, Y.B.; Jun, J.E.; Ahn, J.; Bae, J.C.; Jin, S.M.; Hur, K.Y.; Jee, J.H.; Lee, M.K.; et al. Relationship between relative skeletal muscle mass and non-alcoholic fatty liver disease: A 7-year longitudinal study. Hepatology 2018. [CrossRef] [PubMed]

11. Koo, B.K.; Kim, D.; Joo, S.K.; Kim, J.H.; Chang, M.S.; Kim, B.G.; Lee, K.L.; Kim, W. Sarcopenia is an independent risk factor for non-alcoholic steatohepatitis and significant fibrosis. J. Hepatol. 2017, 66, 123-131. [CrossRef] [PubMed]

12. Petta, S.; Ciminnisi, S.; Di Marco, V.; Cabibi, D.; Cammà, C.; Licata, A.; Marchesini, G.; Craxi, A. Sarcopenia is associated with severe liver fibrosis in patients with non-alcoholic fatty liver disease. Aliment. Pharmacol. Ther. 2017, 45, 510-518. [CrossRef] [PubMed]

13. Rossi, P.; Marzani, B.; Giardina, S.; Negro, M.; Marzatico, F. Human skeletal muscle aging and the oxidative system: Cellular events. Curr. Aging Sci. 2008, 1, 182-191. [CrossRef] [PubMed]

14. Flannery, C.; Dufour, S.; Rabøl, R.; Shulman, G.I.; Petersen, K.F. Skeletal muscle insulin resistance promotes increased hepatic de novo lipogenesis, hyperlipidemia, and hepatic steatosis in the elderly. Diabetes 2012, 61, 2711-2717. [CrossRef] [PubMed]

15. Hong, H.C.; Hwang, S.Y.; Choi, H.Y.; Yoo, H.J.; Seo, J.A.; Kim, S.G.; Kim, N.H.; Baik, S.H.; Choi, D.S.; Choi, K.M. Relationship between sarcopenia and nonalcoholic fatty liver disease: The Korean Sarcopenic Obesity Study. Hepatology 2014, 59, 1772-1778. [CrossRef] [PubMed]

16. De Bandt, J.P. Leucine and mammalian target of rapamycin-dependent activation of muscle protein synthesis in aging. J. Nutr. 2016, 146, 2616S-2624S. [CrossRef] [PubMed]

17. Boucher, J.; Kleinridders, A.; Kahn, C.R. Insulin receptor signaling in normal and insulin-resistant states. Cold Spring Harb. Perspect. Biol. 2014, 6, a009191. [CrossRef] [PubMed]

18. Gryson, C.; Walrand, S.; Giraudet, C.; Rousset, P.; Migné, C.; Bonhomme, C.; Ruyet, P.L.; Boirie, Y. "Fast proteins" with a unique essential amino acid content as an optimal nutrition in the elderly: Growing evidence. Clin. Nutr. 2014, 33, 642-648. [CrossRef] [PubMed]

19. Muniyappa, R.; Quon, M.J. Insulin action and insulin resistance in vascular endothelium. Curr. Opin. Clin. Nutr. Metab. Care 2007, 10, 523-530. [CrossRef] [PubMed]

20. Song, Y.H.; Song, J.L.; Delafontaine, P.; Godard, M.P. The therapeutic potential of IGF-I in skeletal muscle repair. Trends Endocrinol. Metab. 2013, 24, 310-319. [CrossRef] [PubMed] 
21. Short, K.R.; Irving, B.A.; Basu, A.; Johnson, C.M.; Nair, K.S.; Basu, R. Effects of type 2 diabetes and insulin on whole-body, splanchnic, and leg protein metabolism. J. Clin. Endocrinol. Metab. 2012, 97, 4733-4741. [CrossRef] [PubMed]

22. Masgrau, A.; Mishellany-Dutour, A.; Murakami, H.; Beaufrère, A.M.; Walrand, S.; Giraudet, C.; Migne, C.; Gerbaix, M.; Metz, L.; Courteix, D.; et al. Time-course changes of muscle protein synthesis associated with obesity-induced lipotoxicity. J. Physiol. 2012, 590, 5199-5210. [CrossRef] [PubMed]

23. Shen, H.; Eguchi, K.; Kono, N.; Fujiu, K.; Matsumoto, S.; Shibata, M.; Oishi-Tanaka, Y.; Komuro, I.; Arai, H.; Nagai, R.; et al. Saturated fatty acid palmitate aggravates neointima formation by promoting smooth muscle phenotypic modulation. Arterioscler. Thromb. Vasc. Biol. 2013, 33, 2596-2607. [CrossRef] [PubMed]

24. Ibrahim, Z.A.; Armour, C.L.; Phipps, S.; Sukkar, M.B. RAGE and TLRs: Relatives, friends or neighbours? Mol. Immunol. 2013, 56, 739-744. [CrossRef] [PubMed]

25. Howard, A.C.; McNeil, A.K.; Xiong, F.; Xiong, W.C.; McNeil, P.L. A novel cellular defect in diabetes: Membrane repair failure. Diabetes 2011, 60, 3034-3043. [CrossRef] [PubMed]

26. Trayhurn, P. Hypoxia and adipocyte physiology: Implications for adipose tissue dysfunction in obesity. Annu. Rev. Nutr. 2014, 34, 207-236. [CrossRef] [PubMed]

27. Jegatheesan, P.; Beutheu, S.; Ventura, G.; Nubret, E.; Sarfati, G.; Bergheim, I.; De Bandt, J.P. Citrulline and nonessential amino acids prevent fructose-induced nonalcoholic fatty liver disease in rats. J. Nutr. 2015, 145, 2273-2279. [CrossRef] [PubMed]

28. Fuchs, C.D.; Claudel, T.; Trauner, M. Role of metabolic lipases and lipolytic metabolites in the pathogenesis of NAFLD. Trends Endocrinol. Metab. 2014, 25, 576-585. [CrossRef] [PubMed]

29. Bizeau, M.E.; Pagliassotti, M.J. Hepatic adaptations to sucrose and fructose. Metabolism 2005, 54, 1189-1201. [CrossRef] [PubMed]

30. Roncal-Jimenez, C.A.; Lanaspa, M.A.; Rivard, C.J.; Nakagawa, T.; Sanchez-Lozada, L.G.; Jalal, D.; Andres-Hernando, A.; Tanabe, K.; Madero, M.; Li, N.; et al. Sucrose induces fatty liver and pancreatic inflammation in male breeder rats independent of excess energy intake. Metabolism 2011, 60, 1259-1270. [CrossRef] [PubMed]

31. Lecoultre, V.; Egli, L.; Carrel, G.; Theytaz, F.; Kreis, R.; Schneiter, P.; Boss, A.; Zwygart, K.; Le, K.A.; Bortolotti, M.; et al. Effects of fructose and glucose overfeeding on hepatic insulin sensitivity and intrahepatic lipids in healthy humans. Obesity 2013, 21, 782-785. [CrossRef] [PubMed]

32. Volynets, V.; Machann, J.; Küper, M.A.; Maier, I.B.; Spruss, A.; Königsrainer, A.; Bischoff, S.C.; Bergheim, I. A moderate weight reduction through dietary intervention decreases hepatic fat content in patients with non-alcoholic fatty liver disease (NAFLD): A pilot study. Eur. J. Nutr. 2013, 52, 527-535. [CrossRef] [PubMed]

33. Jegatheesan, P.; De Bandt, J.P. Fructose and NAFLD: The multifaceted aspects of fructose metabolism. Nutrients 2017, 9, E230. [CrossRef] [PubMed]

34. Malhi, H.; Kaufman, R.J. Endoplasmic reticulum stress in liver disease. J. Hepatol. 2011, 54, 795-809. [CrossRef] [PubMed]

35. Collison, K.S.; Saleh, S.M.; Bakheet, R.H.; Al-Rabiah, R.K.; Inglis, A.L.; Makhoul, N.J.; Maqbool, Z.M.; Zaidi, M.Z.; AI-Johi, M.A.; AI-Mohanna, F.A. Diabetes of the liver: The link between nonalcoholic fatty liver disease and HFCS-55. Obesity 2009, 17, 2003-2013. [CrossRef] [PubMed]

36. Stefan, N.; Häring, H.U. The role of hepatokines in metabolism. Nat. Rev. Endocrinol. 2013, 9, 144-152. [CrossRef] [PubMed]

37. Jung, T.W.; Lee, S.Y.; Hong, H.C.; Choi, H.Y.; Yoo, H.J.; Baik, S.H.; Choi, K.M. AMPK activator-mediated inhibition of endoplasmic reticulum stress ameliorates carrageenan-induced insulin resistance through the suppression of selenoprotein P in HepG2 hepatocytes. Mol. Cell. Endocrinol. 2014, 382, 66-73. [CrossRef] [PubMed]

38. Gatineau, E.; Savary-Auzeloux, I.; Migné, C.; Polakof, S.; Dardevet, D.; Mosoni, L. Chronic intake of sucrose accelerates sarcopenia in older male rats through alterations in insulin sensitivity and muscle protein synthesis. J. Nutr. 2015, 145, 923-930. [CrossRef] [PubMed]

39. Stanhope, K.L.; Schwarz, J.M.; Keim, N.L.; Griffen, S.C.; Bremer, A.A.; Graham, J.L.; et al. Consuming fructose-sweetened, not glucose-sweetened, beverages increases visceral adiposity and lipids and decreases insulin sensitivity in overweight/obese humans. J. Clin. Investig. 2009, 119, 1322-1334. [CrossRef] [PubMed] 
40. Laclaustra, M.; Rodriguez-Artalejo, F.; Guallar-Castillon, P.; Banegas, J.R.; Graciani, A.; Garcia-Esquinas, E.; Ordovas, J.; Lopez-Garcia, E. Prospective association between added sugars and frailty in older adults. Am. J. Clin. Nutr. 2018, 107, 772-779. [CrossRef] [PubMed]

41. Jelenik, T.; Kaul, K.; Séquaris, G.; Flögel, U.; Phielix, E.; Kotzka, J.; Knebel, B.; Fahlbusch, P.; Horbelt, T.; Lehr, S.; et al. Mechanisms of insulin resistance in primary and secondary nonalcoholic fatty liver. Diabetes 2017, 66, 2241-2253. [CrossRef] [PubMed]

42. Koehler, E.; Swain, J.; Sanderson, S.; Krishnan, A.; Watt, K.; Charlton, M. Growth hormone, dehydroepiandrosterone and adiponectin levels in non-alcoholic steatohepatitis: An endocrine signature for advanced fibrosis in obese patients. Liver Int. 2012, 32, 279-286. [CrossRef] [PubMed]

43. Chishima, S.; Kogiso, T.; Matsushita, N.; Hashimoto, E.; Tokushige, K. The relationship between the growth hormone/insulin-like growth factor system and the histological features of nonalcoholic fatty liver disease. Intern. Med. 2017, 56, 473-480. [CrossRef] [PubMed]

44. Poggiogalle, E.; Lubrano, C.; Gnessi, L.; Mariani, S.; Lenzi, A.; Donini, L.M. Fatty liver index associates with relative sarcopenia and GH/ IGF-1 status in obese subjects. PLoS ONE 2016, 11, e0145811. [CrossRef] [PubMed]

45. Runchey, S.S.; Boyko, E.J.; Ioannou, G.N.; Utzschneider, K.M. Relationship between serum circulating insulin-like growth factor-1 and liver fat in the United States. J. Gastroenterol. Hepatol. 2014, 29, 589-596. [CrossRef] [PubMed]

46. Jegatheesan, P.; Surowska, A.; Campos, V.; Cros, J.; Stefanoni, N.; Rey, V.; Schneiter, P.; De Bandt, J.P.; Tappy, L. Dietary protein content modulates the amino-acid and IGF1 responses to sucrose overfeeding in humans. Clin. Nutr. 2017, 36, S285-S286. [CrossRef]

47. Cabrera, D.; Ruiz, A.; Cabello-Verrugio, C.; Brandan, E.; Estrada, L.; Pizarro, M.; Solis, N.; Torres, J.; Barrera, F; Arrese, M. Diet-induced nonalcoholic fatty liver disease is associated with sarcopenia and decreased serum insulin-like growth factor-1. Dig. Dis. Sci. 2016, 61, 3190-3198. [CrossRef] [PubMed]

48. Marchand, A.; Tomkiewicz, C.; Magne, L.; Barouki, R.; Garlatti, M. Endoplasmic reticulum stress induction of insulin-like growth factor-binding protein-1 involves ATF4. J. Biol. Chem. 2006, 281, 19124-19133. [CrossRef] [PubMed]

49. Crossey, P.A.; Jones, J.S.; Miell, J.P. Dysregulation of the insulin/IGF binding protein-1 axis in transgenic mice is associated with hyperinsulinemia and glucose intolerance. Diabetes 2000, 49, 457-465. [CrossRef] [PubMed]

50. Theytaz, F.; Noguchi, Y.; Egli, L.; Campos, V.; Buehler, T.; Hodson, L.; Patterson, B.W.; Nishikata, N.; Kreis, R.; Mittendorfer, B.; et al. Effects of supplementation with essential amino acids on intrahepatic lipid concentrations during fructose overfeeding in humans. Am. J. Clin. Nutr. 2012, 96, 1008-1016. [CrossRef] [PubMed]

51. Gaggini, M.; Carli, F.; Rosso, C.; Buzzigoli, E.; Marietti, M.; Della Latta, V.; Ciociaro, D.; Abate, M.L.; Gambino, R.; Cassader, M.; et al. Altered amino acid concentrations in NAFLD: Impact of obesity and insulin resistance. Hepatology 2018, 67, 145-158. [CrossRef] [PubMed]

52. Meex, R.C.R.; Watt, M.J. Hepatokines: Linking nonalcoholic fatty liver disease and insulin resistance. Nat. Rev. Endocrinol. 2017, 13, 509-520. [CrossRef] [PubMed]

53. Meex, R.C.; Hoy, A.J.; Morris, A.; Brown, R.D.; Lo, J.C.Y.; Burke, M.; Goode, R.J.A.; Kingwell, B.A.; Kraakman, M.J.; Febbraio, M.A.; et al. Fetuin B is a secreted hepatocyte factor linking steatosis to impaired glucose metabolism. Cell. Metab. 2015, 22, 1078-1089. [CrossRef] [PubMed]

54. Musso, G.; Paschetta, E.; Gambino, R.; Cassader, M.; Molinaro, F. Interactions among bone, liver, and adipose tissue predisposing to diabesity and fatty liver. Trends Mol. Med. 2013, 19, 522-535. [CrossRef] [PubMed]

55. Ou, H.Y.; Wu, H.T.; Hung, H.C.; Yang, Y.C.; Wu, J.S.; Chang, C.J. Endoplasmic reticulum stress induces the expression of fetuin-A to develop insulin resistance. Endocrinology 2012, 153, 2974-2984. [CrossRef] [PubMed]

56. Srinivas, P.R.; Wagner, A.S.; Reddy, L.V.; Deutsch, D.D.; Leon, M.A.; Goustin, A.S.; Grunberger, G. Serum alpha 2-HS-glycoprotein is an inhibitor of the human insulin receptor at the tyrosine kinase level. Mol. Endocrinol. 1993, 7, 1445-1455. [PubMed]

57. Liu, J.; Xu, Y.; Hu, Y.; Wang, G. The role of fibroblast growth factor 21 in the pathogenesis of non-alcoholic fatty liver disease and implications for therapy. Metabolism 2015, 64, 380-390. [CrossRef] [PubMed] 
58. Hong, S.H.; Ahmadian, M.; Yu, R.T.; Atkins, A.R.; Downes, M.; Evans, R.M. Nuclear receptors and metabolism: From feast to famine. Diabetologia 2014, 57, 860-867. [CrossRef] [PubMed]

59. Abdul-Wahed, A.; Guilmeau, S.; Postic, C. Sweet sixteenth for ChREBP: Established roles and future goals. Cell. Metab. 2017, 26, 324-341. [CrossRef] [PubMed]

60. Jiang, S.; Yan, C.; Fang, Q.; Shao, M.; Zhang, Y.; Liu, Y.; Deng, Y.; Shan, B.; Liu, J.; Li, H.; et al. Fibroblast growth factor 21 is regulated by the IRE1 $\alpha-\mathrm{XBP} 1$ branch of the unfolded protein response and counteracts endoplasmic reticulum stress-induced hepatic steatosis. J. Biol. Chem. 2014, 289, 29751-29765. [CrossRef] [PubMed]

61. Chukijrungroat, N.; Khamphaya, T.; Weerachayaphorn, J.; Songserm, T.; Saengsirisuwan, V. Hepatic FGF21 mediates sex differences in high-fat high-fructose diet-induced fatty liver. Am. J. Physiol. Endocrinol. Metab. 2017, 313, E203-E212. [CrossRef] [PubMed]

62. Staiger, H.; Keuper, M.; Berti, L.; Hrabe de Angelis, M.; Häring, H.U. Fibroblast Growth Factor 21-Metabolic role in mice and men. Endocr. Rev. 2017, 38, 468-488. [CrossRef] [PubMed]

63. Wu, H.T.; Ou, H.Y.; Hung, H.C.; Su, Y.C.; Lu, F.H.; Wu, J.S.; Yang, Y.C.; Wu, C.L.; Chang, C.J. A novel hepatokine, HFREP1, plays a crucial role in the development of insulin resistance and type 2 diabetes. Diabetologia 2016, 59, 1732-1742. [CrossRef] [PubMed]

64. Jung, T.W.; Chung, Y.H.; Kim, H.C.; Abd El-Aty, A.M.; Jeong, J.H. Hyperlipidemia-induced hepassocin in the liver contributes to insulin resistance in skeletal muscle. Mol. Cell. Endocrinol. 2018, 470, 26-33. [CrossRef] [PubMed]

65. Allaman, I.; Bélanger, M.; Magistretti, P.J. Methylglyoxal, the dark side of glycolysis. Front. Neurosci. 2015, 9, 23. [CrossRef] [PubMed]

66. Dhar, I.; Dhar, A.; Wu, L.; Desai, K. Arginine attenuates methylglyoxal- and high glucose-induced endothelial dysfunction and oxidative stress by an endothelial nitric-oxide synthase-independent mechanism. J. Pharmacol. Exp. Ther. 2012, 342, 196-204. [CrossRef] [PubMed]

67. Dhar, I.; Dhar, A.; Wu, L.; Desai, K.M. Increased methylglyoxal formation with upregulation of renin angiotensin system in fructose fed Sprague Dawley rats. PLoS ONE 2013, 8, e74212. [CrossRef] [PubMed]

68. Abdelmalek, M.F.; Lazo, M.; Horska, A.; Bonekamp, S.; Lipkin, E.W.; Balasubramanyam, A.; Bantle, J.P.; Johnson, R.J.; Diehl, A.M.; Clark, J.M.; et al. Higher dietary fructose is associated with impaired hepatic adenosine triphosphate homeostasis in obese individuals with type 2 diabetes. Hepatology 2012, 56, 952-960. [CrossRef] [PubMed]

69. Zhu, Y.; Hu, Y.; Huang, T.; Zhang, Y.; Li, Z.; Luo, C.; Luo, Y.; Yuan, H.; Hisatome, I.; Yamamoto, T.; et al. High uric acid directly inhibits insulin signalling and induces insulin resistance. Biochem. Biophys. Res. Commun. 2014, 447, 707-714. [CrossRef] [PubMed]

70. Fabbrini, E.; Serafini, M.; Colic Baric, I.; Hazen, S.L.; Klein, S. Effect of plasma uric acid on antioxidant capacity, oxidative stress, and insulin sensitivity in obese subjects. Diabetes 2014, 63, 976-981. [CrossRef] [PubMed]

71. Beavers, K.M.; Beavers, D.P.; Serra, M.C.; Bowden, R.G.; Wilson, R.L. Low relative skeletal muscle mass indicative of sarcopenia is associated with elevations in serum uric acid levels: Findings from NHANES III. J. Nutr. Health Aging 2009, 13, 177-182. [CrossRef] [PubMed]

72. Ticinesi, A.; Lauretani, F.; Milani, C.; Nouvenne, A.; Tana, C.; Del Rio, D.; Maggio, M.; Ventura, M.; Meschi, T. Aging gut microbiota at the cross-road between nutrition, physical frailty, and sarcopenia: Is there a gut-muscle axis? Nutrients 2017, 9, 1303. [CrossRef] [PubMed]

(C) 2018 by the authors. Licensee MDPI, Basel, Switzerland. This article is an open access article distributed under the terms and conditions of the Creative Commons Attribution (CC BY) license (http:/ / creativecommons.org/licenses/by/4.0/). 
Article

\title{
Dissociation of Fatty Liver and Insulin Resistance in I148M PNPLA3 Carriers: Differences in Diacylglycerol (DAG) FA18:1 Lipid Species as a Possible Explanation
}

\author{
Andras Franko 1,2,3,*(D), Dietrich Merkel ${ }^{4}$, Marketa Kovarova 1,2,3, Miriam Hoene ${ }^{1}$, \\ Benjamin A. Jaghutriz 1,2,3, Martin Heni ${ }^{1,2,3}$, Alfred Königsrainer ${ }^{5}$, Cyrus Papan ${ }^{4}$, \\ Stefan Lehr ${ }^{3,6}\left(\mathbb{D}\right.$, Hans-Ulrich Häring ${ }^{1,2,3}$ and Andreas Peter 1,2,3 \\ 1 Department of Internal Medicine IV, Division of Endocrinology, Diabetology, Angiology, \\ Nephrology and Clinical Chemistry, University Hospital Tübingen, 72076 Tübingen, Germany; \\ kovarova-marketa@seznam.cz (M.K.); miriam.hoene@med.uni-tuebingen.de (M.H.); \\ benjamin.jaghutriz@med.uni-tuebingen.de (B.A.J.); martin.heni@med.uni-tuebingen.de (M.H.); \\ hans-ulrich.haering@med.uni-tuebingen.de (H.-U.H.); andreas.peter@med.uni-tuebingen.de (A.P.) \\ 2 Institute for Diabetes Research and Metabolic Diseases of the Helmholtz Center Munich, \\ University of Tübingen, 72076 Tübingen, Germany \\ 3 German Center for Diabetes Research (DZD e.V.), 85764 Neuherberg, Germany; \\ stefan.lehr@ddz.uni-duesseldorf.de \\ 4 Sciex Germany GmbH, 64293 Darmstadt, Germany; dietrich.merkel@sciex.com (D.M.); \\ cyrus.papan@sciex.com (C.P.) \\ 5 Department of General, Visceral and Transplant Surgery, University Hospital Tübingen, \\ 72076 Tübingen, Germany; alfred.koenigsrainer@med.uni-tuebingen.de \\ 6 Institute for Clinical Biochemistry and Pathobiochemistry of DDZ, University of Düsseldorf, \\ 40225 Düsseldorf, Germany \\ * Correspondence: andras.franko@med.uni-tuebingen.de; Tel.: +49-7071-29-85255
}

Received: 6 August 2018; Accepted: 13 September 2018; Published: 17 September 2018

\begin{abstract}
Fatty liver is tightly associated with insulin resistance and the development of type 2 diabetes. I148M variant in patatin-like phospholipase domain-containing protein 3 (PNPLA3) gene is associated with high liver fat but normal insulin sensitivity. The underlying mechanism of the disassociation between high liver fat but normal insulin sensitivity remains obscure. We investigated the effect of I148M variant on hepatic lipidome of subjects with or without fatty liver, using the Lipidyzer method. Liver samples of four groups of subjects consisting of normal liver fat with wild-type PNPLA3 allele (group 1); normal liver fat with variant PNPLA3 allele (group 2); high liver fat with wild-type PNPLA3 allele (group 3); high liver fat with variant PNPLA3 allele (group 4); were analyzed. When high liver fat to normal liver fat groups were compared, wild-type carriers (group 3 vs. group 1) showed similar lipid changes compared to I148M PNPLA3 carriers (group 4 vs. group 2). On the other hand, in wild-type carriers, increased liver fat significantly elevated the proportion of specific DAGs (diacylglycerols), mostly DAG (FA18:1) which, however, remained unchanged in I148M PNPLA3 carriers. Since DAG (FA18:1) has been implicated in hepatic insulin resistance, the unaltered proportion of DAG (FA18:1) in I148M PNPLA3 carriers with fatty liver may explain the normal insulin sensitivity in these subjects.
\end{abstract}

Keywords: NAFLD; liver; PNPLA3; diacylglycerol; lipidomics 


\section{Introduction}

Nonalcoholic fatty liver disease (NAFLD) is characterized by elevated hepatic lipid content [1]. NAFLD is claimed to be a benign illness, however, it can further develop to nonalcoholic steatohepatitis (NASH), liver fibrosis, cirrhosis and hepatocellular carcinoma [2]. The prevalence of NAFLD is continuously increasing, and currently it is estimated to be higher than $20 \%$ in industrialized countries [3]. Despite this high prevalence of NAFLD and its complication NASH, there is no established effective drug therapy, which is generally approved for these illnesses [4]. NAFLD/NASH patients are advised to lose weight with lifestyle intervention (mostly consisting of healthy diet and exercise [4]), however, not all patients benefit from these interventions [5]. To treat NAFLD/NASH, several drugs have been shown to be beneficial in animal models [6,7], and novel activators for peroxisome proliferator-activated receptor (PPAR) (elafibranor) and farnesoid X receptor (FXR) (obeticholic acid) are currently under phase 3 studies in human cohorts with promising results [4]. NAFLD is a strong determinant of insulin sensitivity and the development of type 2 diabetes, however, some distinct genetic causes for the dissociation of liver fat content and insulin sensitivity have been identified [1].

The rs738409 C>G single nucleotide polymorphism (SNP) in the patatin-like phospholipase domain-containing protein 3 (PNPLA3) gene is a common inherited trait, which results in an amino acid exchange I148M leading to a functional mutation of PNPLA3 [8]. At this position, both hetero(I148M) and homozygous (M148M) variants are described. The prevalence of PNPLA3 variants vary due to ethnicity of the population [9] and 34-37\% (I148M) and 4-9\% (M148M) are described in studies analyzing German or European-American populations, respectively $[8,10]$. Several studies demonstrated that I148M PNPLA3 carriers showed an altered metabolic phenotype on nutritional challenges compared to wild-type subjects. Dietary intake of carbohydrates was shown to modify the association between PNPLA3 genotype and circulating triglyceride levels [11]. Carbohydrate overfeeding led to an increased de-novo lipogenesis in proportion to the increase in liver fat and serum triglycerides in subjects with I148I carriers, which however, was not observed in M148M carriers [12]. Furthermore, the M148M PNPLA3 variant influenced the changes in liver fat and docosahexaenoic acid tissue enrichment during a 15-18 months addition of omega 3 fatty acids [13]. The presence of rs738409 SNP was positively associated with elevated liver fat, however, carriers do not show insulin resistance $[8,10,14]$. On the other hand, some subjects carrying the I148M variant show normal liver fat content, and the function of PNPLA3 in these subjects is not studied yet. PNPLA3 has several enzyme activities, it was reported to be involved in lipid hydrolysis (as a triacylglycerol lipase) and synthesis (as a lysophosphatidic acyltransferase) [15]. The I148M variant is claimed to show lower lipolysis of hepatic triacylglycerols (TAGs) [16] and elevated hepatic TAG synthesis [17]. However, for subjects carrying I148M PNPLA3 variant a lower de novo lipogenesis is also reported [18]. The discrepancy between high liver TAG level and normal insulin sensitivity in I148M carriers is not resolved yet [19], but altered hepatic TAG pattern, especially long-chain polyunsaturated fatty acid content, is reported in rodent and human studies analyzing I148M PNPLA3 carriers $[14,15,20]$. Several lipid species are implicated in the state of insulin resistance in patients with NAFLD [21]. Elevated ceramide [15] or high diacylglycerol (DAG) content [22,23] have been found in the liver of rodents and human subjects with insulin resistance. Furthermore, quantitative measurements of unique lipid species have been previously shown to broaden our knowledge to understand complex diseases, such as cystic fibrosis, NAFLD and type 1 diabetes and pave the way for identifying new lipid biomarkers [24-27].

In order (i) to study the function of I148M PNPLA3 variant in subjects with normal and high liver fat and, (ii) to analyze the key metabolic lipids (also including detailed measurements of individual ceramide and diacylglycerol lipid species) possibly evoking insulin resistance in wild-type but not in I148M PNPLA3 carriers, we studied the liver of subjects with normal and high hepatic TAG content with wild-type or I148M (heterozygous), as well as M148M (homozygous) PNPLA3 variants. To examine the lipid profiles, we decided to perform an unbiased lipidomic analysis using the Lipidyzer 
platform, which was originally established for plasma samples [28], therefore, we applied it with an adapted protocol for solid tissue lysates.

\section{Materials and Methods}

\subsection{Human Liver Samples, Total Liver TAG Measurement, and PNPLA3 Genotyping}

For the analysis of liver tissue samples, a cohort of European descendent men and women undergoing liver surgery at the Department of General, Visceral, and Transplant Surgery at the University Hospital of Tübingen was included in the present study. None of the patients were diagnosed with an abuse of alcohol, however, no detailed data on alcohol consumption was consistently collected. The liver tissue was collected during hepatic surgery that was performed for different reasons, e.g., hepatic hemangioma, curative resection of hepatic metastases of colorectal malignancies or hepatocellular carcinoma. Patients fasted overnight before collection of liver samples. Exclusion criteria were viral hepatitis infection and liver cirrhosis. Informed, written consent was obtained from all participants, and the Ethics Committee of the University of Tübingen approved the protocol (239/2013BO1) according to the Declaration of Helsinki. Liver samples taken from normal, non-diseased tissue, were quickly frozen in liquid nitrogen and stored at $-80^{\circ} \mathrm{C}$. To measure total TAG content, liver tissue samples were homogenized in phosphate buffered saline containing $1 \%$ Triton X-100 with a TissueLyser (Qiagen, Hilden, Germany) and determined as described previously [29,30]. In order to match subjects for similar body weight, BMI (body mass index) and age as well as for different liver TAG content, subjects showing less than 3.0\% liver TAG content were classified as normal TAG group and subjects showing more than $4.3 \%$ liver TAG were classified as high TAG group. For PNPLA3 genotyping, total DNA was isolated from whole blood using a DNA isolation kit (NucleoSpin, Macherey and Nagel, Düren, Germany). The I148M PNPLA3 variations were genotyped using Sequenom's massARRAY System with iPLEX software (Sequenom, Hamburg, Germany) as described previously [14]. Plasma ALT levels were measured with routine clinical chemistry [10].

\subsection{Lipidyzer Platform}

The Lipidyzer ${ }^{\mathrm{TM}}$ platform (SCIEX, Darmstadt, Germany) was used for the whole lipid analysis work flow. Briefly, $10 \mathrm{mg}$ liver was solubilized in $100 \mu \mathrm{L}$ internal standards (IS, Avanti Polar Lipids, Inc., AL, USA) and $200 \mu \mathrm{L}$ of $75 \%$ methanol was added and hepatic lipids were extracted using methyl tert-butyl ether (MTBE) as described previously [31]. The following isotopes labeled internal standards were used dCER(d16:0), dCE(16:0), dCE(16:1), dCE(18:1), dCE(18:2), dCE(20:3), dCE(20:4), dCE(20:5), dCE(22:6), dDAG(16:0/16:0), dDAG(16:0/18:0), dDAG(16:0/18:1), dDAG(16:0/18:2), dDAG(16:0/18:3), $\operatorname{dDAG}(16: 0 / 20: 4), \operatorname{dDAG}(16: 0 / 20: 5), \operatorname{dDAG}(16: 0 / 22: 6), \operatorname{dFFA}(16: 0), \operatorname{dFFA}(17: 1), \operatorname{dLPC}(16: 0)$, dLPE(18:0), dPC(16:0/16:1), dPC(16:0/18:1), dPC(16:0/18:2), $\operatorname{dPC}(16: 0 / 18: 3), \operatorname{dPC}(16: 0 / 20: 3)$, $\operatorname{dPC}(16: 0 / 20: 4), \operatorname{dPC}(16: 0 / 20: 5), \operatorname{dPC}(16: 0 / 22: 4), \operatorname{dPC}(16: 0 / 22: 5), \operatorname{dPC}(16: 0 / 22: 6), \operatorname{dPE}(18: 0 / 18: 1)$, $\mathrm{dPE}(18: 0 / 18: 2), \operatorname{dPE}(18: 0 / 18: 3), \operatorname{dPE}(18: 0 / 20: 3), \operatorname{dPE}(18: 0 / 20: 4), \operatorname{dPE}(18: 0 / 20: 5), \operatorname{dPE}(18: 0 / 22: 5)$, dPE(18:0/22:6), dSM(16:0), dSM(18:1), dSM(24:0), dSM(24:1), dTAG50:1-FA16:0, dTAG52:1-FA18:0, dTAG52:2-FA18:1, dTAG52:3-FA18:2, dTAG52:4-FA18:3， dTAG54:4-FA20:3， dTAG54:5-FA20:4, dTAG56:7-FA22:6, dDCER(16:0), dHCER(16:0), and dLCER(16:0). For each injection, $50 \mu \mathrm{L}$ of extracted lipid sample was introduced by flow injection (FIA) using a Nexera X2 system (Shimadzu Germany $\mathrm{GmbH}$, Duisburg, Germany), equipped with a $50 \mu \mathrm{L}$-sample loop. A Lipidyzer ${ }^{\mathrm{TM}}$ included $750 \times 0.05$ mm PEEKsil ${ }^{\mathrm{TM}}$ (Trajan Scientific Europe Ltd., Milton Keynes, UK) sample tubing was used to connect the autosampler valve with the grounding union on the electrospray ionization (ESI) source, and a $350 \times 0.05 \mathrm{~mm}$ PEEKsil ${ }^{\mathrm{TM}}$ (Trajan Scientific Europe Ltd, Milton Keynes, UK) sample tubing was used to connect the grounding union with the ESI electrode having $65 \mu \mathrm{m}$ inner diameter. The flow profile for the flow injection was determined by the Lipidyzer ${ }^{\mathrm{TM}}$ acquisition method with a flow rate during the data acquisition period being $7 \mu \mathrm{L} / \mathrm{min}$. The mass spectrometry analysis was performed on a Lipidyzer ${ }^{\mathrm{TM}}$ Platform, including the Sciex QTRAP ${ }^{\circledR}$ (SCIEX, Darmstadt, Germany) 5500 
system equipped with SelexION ${ }^{\circledR}$ (SCIEX, Darmstadt, Germany) Technology (differential mobility separation, DMS). Multiple reaction monitoring (MRM) was used to target and quantitate several hundreds of lipid molecular species from 13 different lipid classes. All samples were first measured in positive and negative polarity with SelexION separation, followed by measurement without SelexION separation. The acquisition time per sample took approximately $25 \mathrm{~min}$ for the complete acquisition. All data was acquired and processed automatically using the Lipid Manager Workflow software (SCIEX, Darmstadt, Germany). This provides the following data tables: (i) quantitative results for each lipid class as a sum of individual species; (ii) mole percent composition obtained computationally from lipid molecular species data; and (iii) accurate lipid species compositions.

\subsection{Data Evaluation}

Missing values, which were not possible to measure and showed zero values, were handled as follows: From the complete data set, lipids, which showed higher values than zero at least in $50 \%$ of any group were kept, otherwise they were discarded. To determine whether the groups separate from each other according to PNPLA3 genotype or hepatic TAG content, multivariate partial least squares discriminant analysis (PLS-DA) were performed using soft independent modeling of class analogy (SIMCA, Umetrics, Umea, Sweden). The 761 individual lipid species and the 84 sum of lipid classes were summed up and these 845 lipid values were logarithmic transformed and were statistically evaluated, as written below.

\subsection{Statistics}

To determine statistical different lipid species caused by increased liver fat, group 3 (high liver fat, wild-type PNPLA3 allele) was compared to group 1 (normal liver fat, wild-type PNPLA3 allele) as well as group 4 (high liver fat, variant PNPLA3 allele) was compared to group 2 (normal liver fat, variant PNPLA3 allele) using GraphPad Prism (7.03). Multiple t-tests were applied with Benjamini-Hochberg correction and false discovery rate (FDR) was set $<5 \%$. Furthermore, analysis of variance (ANOVA) with Holm-Sidak's post hoc test were applied as it is indicated.

\section{Results}

\subsection{Characteristics of Study Groups}

To distinguish between the effect of increased liver TAG content and PNPLA3 genotype, subjects were matched for body weight, BMI and age and divided into the following four groups: subjects showing normal liver fat with wild-type PNPLA3 (group 1); normal liver fat with I148M PNPLA3 variant (group 2); high liver fat with wild-type PNPLA3 (group 3) and high liver fat with I148M PNPLA3 variant (group 4) (Table 1).

Our study groups consisted of overweight subjects with similar age, weight, BMI and ALT levels (Table 1). Liver fat was significantly higher in high TAG groups compared to normal groups (group 3 vs. 1 and group 4 vs. 2), but was not different in I148M PNPLA3 carriers compared to wild-type carriers (Table 1). Both variant PNPLA3 groups (groups 2 and 4) consisted of one homozygous M148M (MM) carrier and the rest of the subjects were heterozygous I148M (IM) carriers (Table 1).

In order to study the effect of I148M PNPLA3 variant on hepatic lipid species in subjects with normal and high liver fat, a complete lipid profile was measured using a novel Lipidyzer approach. The following 13 lipid classes were analyzed: triacylglycerols (TAG), diacylglycerols (DAG), free fatty acids (FFA), ceramides (CER), dihydroceramides (DCER), hexosylceramides (HCER), lactosylceramides (LCER), phosphatidylcholines (PC), lysophosphatidylcholines (LPC), phosphatidylethanolamines (PE), lysophosphatidylethanolamines (LPE), cholesterol esters (CE), and sphingomyelins (SM). Among these lipid classes, 761 individual lipid species were measured and 84 sums of individual classes were calculated. In order to investigate whether the hepatic lipid profile of groups with different PNPLA3 genotype or various liver TAG content was different from each other, we first analyzed the data with 
multivariate partial least squares discriminant analysis (PLS-DA). PLS-DA showed that normal TAG, wt PNPLA3 (group 1) and high TAG, wt PNPLA3 (group 3) formed distinct groups, however, normal TAG, var PNPLA3 (group 2) and high TAG, var PNPLA3 (group 4) groups were rather similar taking into account all 761 individual lipid species (Figure 1).

Table 1. Characteristics of study groups.

\begin{tabular}{ccccc}
\hline Characteristic & $\begin{array}{c}\text { Group 1 } \\
\text { Normal TAG, } \\
\text { wt PNPLA3 }\end{array}$ & $\begin{array}{c}\text { Group 2 } \\
\text { Normal TAG, } \\
\text { var PNPLA3 }\end{array}$ & $\begin{array}{c}\text { Group 3 } \\
\text { High TAG, } \\
\text { wt PNPLA3 }\end{array}$ & $\begin{array}{c}\text { Group 4 } \\
\text { High TAG, } \\
\text { var PNPLA3 }\end{array}$ \\
\hline Age (years) & $59.3 \pm 12.6$ & $60.6 \pm 16.4$ & $64.0 \pm 11.8$ & $65.1 \pm 14.5$ \\
Body weight $(\mathrm{kg})$ & $79.3 \pm 9.9$ & $80.3 \pm 13.5$ & $86.2 \pm 11.1$ & $87.1 \pm 13.1$ \\
BMI $\left(\mathrm{kg} / \mathrm{m}^{2}\right)$ & $26.1 \pm 3.2$ & $28.0 \pm 5.3$ & $31.0 \pm 3.4$ & $28.6 \pm 4.0$ \\
ALT $(\mathrm{U} / \mathrm{L})$ & $24.5 \pm 6.5$ & $22.0 \pm 5.2$ & $31.1 \pm 9.3$ & $35.7 \pm 18.6$ \\
Liver fat $(\%)$ & $1.1 \pm 0.8$ & $1.5 \pm 0.7$ & $5.9 \pm 2.0 * * *$ & $7.6 \pm 2.9^{* * * *}$ \\
PNPLA3 ${ }^{\text {148 }}$ (II/IM/MM) $(n)$ & $8 / 0 / 0$ & $0 / 7 / 1$ & $8 / 0 / 0$ & $0 / 6 / 1$ \\
Sex (m/f) $(n)$ & $6 / 2$ & $4 / 4$ & $5 / 3$ & $5 / 2$ \\
Number of subjects $(n)$ & 8 & 8 & 8 & 7 \\
\hline
\end{tabular}

TAG: liver triacylglycerol content; PNPLA3: patatin-like phospholipase domain-containing protein 3; wt: wild-type allele with I148I; var: I148M variants, which encode I148M (heterozygous) or M148M (homozygous) variants, respectively. ALT: alanine aminotransferase, BMI: body mass index. Numbers denote averages \pm standard deviations in the first five lines. ${ }^{* * *}$ denotes significant differences between group 3 vs. 1 or group 4 vs. 2 illustrating the effect of liver TAG content; $p<0.001$. Significance was calculated using ANOVA with Holm-Sidak's post-hoc test and assumed as $p<0.05$. By the comparisons of group 2 vs. 1 and group 4 vs. 3 no significant differences were found for I148M PNPLA3 variant vs. wild-type carriers.

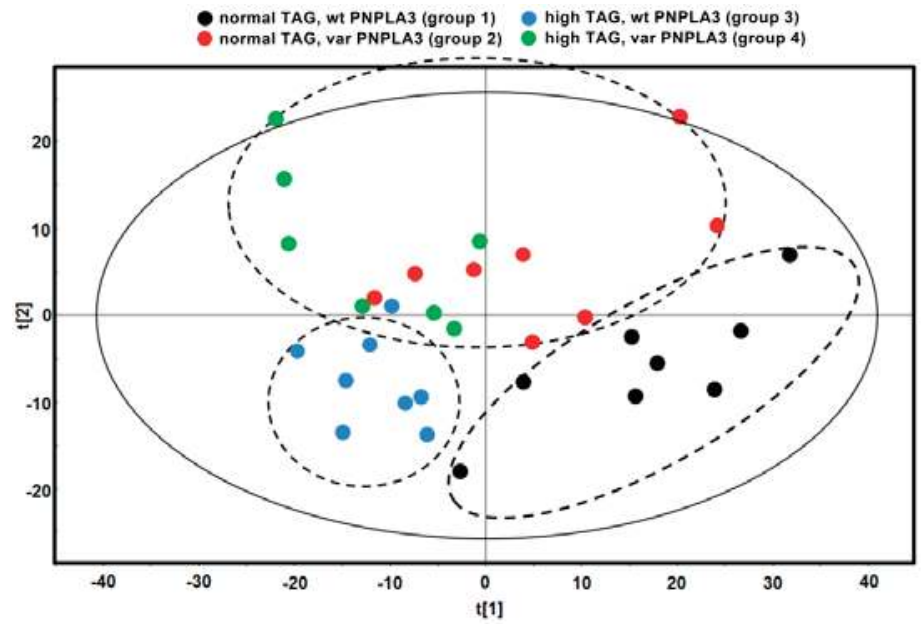

Figure 1. Partial least squares discriminant analysis (PLS-DA) score plot. Each spot represents one liver sample of the denoted group according to component 1 ( $x$ axis) and 2 ( $y$ axis). Dashed lines denote possible separation of the groups taking into account all 761 individual lipid species. TAG: liver triacylglycerol content; wt: wild-type allele with I148I; var: I148M variants, which encode I148M (heterozygous) or M148M (homozygous) variants, respectively.

\subsection{I148M PNPLA3 Variant Does Not Change Relative Total Lipid Contents}

As a next step, the sum of 13 lipid classes was evaluated. With increased liver TAG content, we observed significantly higher relative levels of TAG and DAG lipids (calculated as \% of total lipid content, Table 2). On the other hand, the relative content of FFA, CER, DCER, HCER, PC, LPC, PE, 
LPE, and SM were significantly lower in high TAG vs. normal TAG groups (Table 2). The presence of I148M PNPLA3 variant did not significantly influence the sum of lipid classes (Table 2).

Table 2. Relative lipid contents of individual classes in percent.

\begin{tabular}{ccccc}
\hline $\begin{array}{c}\text { Lipid Class } \\
\%\end{array}$ & $\begin{array}{c}\text { Group 1 } \\
\text { Normal TAG, } \\
\text { wt PNPLA3 }\end{array}$ & $\begin{array}{c}\text { Group 2 } \\
\text { Normal TAG, } \\
\text { var PNPLA3 }\end{array}$ & $\begin{array}{c}\text { Group 3 } \\
\text { High TAG, } \\
\text { wt PNPLA3 }\end{array}$ & $\begin{array}{c}\text { Group 4 } \\
\text { High TAG, } \\
\text { var PNPLA3 }\end{array}$ \\
\hline TAG & $25.63 \pm 12.49$ & $35.22 \pm 15.30$ & $68.64 \pm 11.26^{* * *}$ & $69.82 \pm 11.86^{* * *}$ \\
DAG & $0.60 \pm 0.30$ & $0.57 \pm 0.17$ & $0.92 \pm 0.12^{*}$ & $1.02 \pm 0.20^{* * *}$ \\
FFA & $7.23 \pm 3.57$ & $6.07 \pm 1.71$ & $2.60 \pm 1.12^{* * *}$ & $2.37 \pm 1.01^{* *}$ \\
CER & $0.26 \pm 0.04$ & $0.25 \pm 0.08$ & $0.10 \pm 0.03^{* * *}$ & $0.10 \pm 0.05^{* * *}$ \\
DCER & $0.03 \pm 0.01$ & $0.03 \pm 0.01$ & $0.01 \pm 0.00^{* * * *}$ & $0.01 \pm 0.01^{* * *}$ \\
HCER & $0.07 \pm 0.02$ & $0.05 \pm 0.02$ & $0.03 \pm 0.01^{* * *}$ & $0.02 \pm 0.01^{* *}$ \\
LCER & $0.06 \pm 0.01$ & $0.06 \pm 0.03$ & $0.04 \pm 0.02$ & $0.03 \pm 0.01$ \\
PC & $38.51 \pm 9.74$ & $33.06 \pm 8.63$ & $14.86 \pm 6.41^{* * *}$ & $14.75 \pm 7.83^{* * *}$ \\
LPC & $0.66 \pm 0.22$ & $0.60 \pm 0.14$ & $0.24 \pm 0.14^{* * *}$ & $0.28 \pm 0.17^{* * *}$ \\
PE & $20.43 \pm 5.80$ & $17.84 \pm 5.82$ & $8.19 \pm 3.24^{* * * *}$ & $7.29 \pm 3.10^{* * *}$ \\
LPE & $0.16 \pm 0.05$ & $0.15 \pm 0.03$ & $0.06 \pm 0.03^{* * *}$ & $0.07 \pm 0.04^{* * *}$ \\
CE & $2.45 \pm 0.34$ & $2.84 \pm 0.45$ & $2.73 \pm 0.60$ & $2.86 \pm 0.48$ \\
SM & $3.91 \pm 0.81$ & $3.28 \pm 0.81$ & $1.59 \pm 0.67^{* * * *}$ & $1.37 \pm 0.60^{* * *}$ \\
\hline
\end{tabular}

TAG: triacylglycerols, DAG: diacylglycerols, FFA: free fatty acids, CER: ceramides, DCER: dihydroceramides, HCER: hexosylceramides, LCER: lactosylceramides, PC: phosphatidylcholines, LPC: lysophosphatidylcholines, PE: phosphatidylethanolamines, LPE: lysophosphatidylethanolamines, CE: cholesterol esters, SM: sphingomyelins; wt: wild-type allele with I148I; var: I148M variants, which encode I148M (heterozygous) or M148M (homozygous) variants, respectively. Numbers denote averages \pm standard deviations. ${ }^{*}$ denotes significant differences between group 3 vs. 1 or group 4 vs. 2 illustrating the effect of liver TAG content; ${ }^{*} p<0.05,{ }^{* *} p<0.01,{ }^{* * *} p<0.001$. Significance was calculated using ANOVA with Holm-Sidak's post-hoc test and assumed as $p<0.05$. By the comparisons of group 2 vs. 1 and group 4 vs. 3 no significant differences were found for I148M PNPLA3 variant vs. wild-type carriers.

3.3. Increased Liver Fat Content Is Associated with High Proportion of DAG (FA18:1) Species in Subjects with Wild-Type PNPLA3, However, DAG (FA18:1) Remains Unchanged in I148M PNPLA3 Carriers

In order to study the combination effect of increased liver fat content and PNPLA3 variant on hepatic lipid profile, we next compared the relative proportion of lipid species in high liver fat to normal liver fat groups from subjects with wild-type (group 3 vs. 1) or with I148M PNPLA3 carriers (group 4 vs. 2) (Figure 2 and Supplementary Table S1).

Although in wild-type carriers many individual DAGs decreased with increased hepatic TAG content, the proportion of DAG (C16:0/C18:1), DAG (C18:0/C18:1), DAG (C18:1/C18:1) as well as the sum of DAG (FA18:1) were increased (Figure 2, first diagram, grey arrows). These FA18:1 containing DAGs remained however, unaltered in I148M PNPLA3 variant carriers (Figure 2, second diagram, grey arrows). Furthermore, various individual CE lipids decreased and many individual shorter TAG lipids were elevated in wild-type subjects due to increased liver TAG content; however, these lipid species remained unaltered in I148M PNPLA3 carriers (Supplementary Table S1). These results indicate that subjects, who carry the I148M PNPLA3 variant do not increase the proportion of DAG (FA18:1) lipid levels upon increased hepatic TAG, although many other lipid species altered similarly compared to wild-type carriers. 


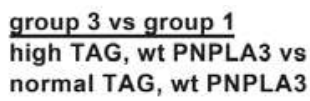

group 4 vs group 2 high TAG, var PNPLA3 vs normal TAG, var PNPLA3

DAG(12:0/18:2)

$\operatorname{DAG}(14: 1 / 18: 1)$

DAG(15:0/18:2)

DAG(16:0/16:1)

DAG(16:0/18:1)

DAG(16:0/18:2)

$\operatorname{DAG}(16: 0 / 20: 3)$

DAG(16:0/20:4)

DAG(16:0/20:5)

$\operatorname{DAG}(16: 0 / 22: 5)$

DAG (16:0/22:6)

DAG(16:1/18:2)

DAG(16:1/20:0)

DAG(18:0/18:1)

DAG(18:0/18:2)

DAG (18:0/22:6)

DAG(18:1/18:1)

DAG(18:1/20:3)

DAG(18:1/20:4)

DAG(18:1/22:5)

DAG(18:1/22:6)

DAG(18:2/20:4)

DAG(20:0/20:0)

DAG(FA14:1)

DAG(FA18:0)

DAG(FA18:1)

DAG(FA18:2)

DAG(FA20:0)

DAG(FA20:3)

DAG(FA20:4)

DAG(FA20:5)

DAG(FA22:5)

DAG(FA22:6)
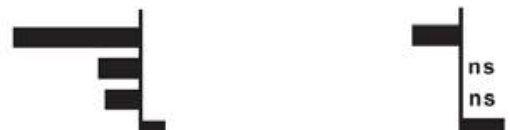

)

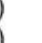

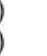

)

1)

)

(6)
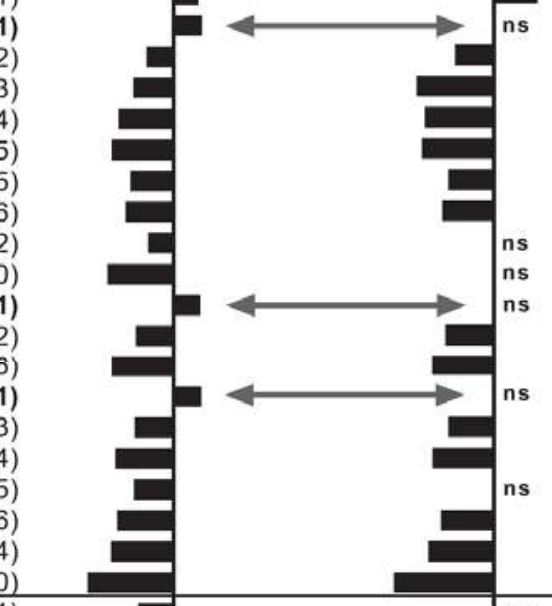

$-10$

Figure 2. Individual DAGs and their sums, which are significantly changed due to high vs. normal TAG level in subjects with wild-type PNPLA3 (first diagram) and I148M PNPLA3 carriers (second diagram). Column diagrams depict linear fold changes calculated from the proportion of relative individual lipid species and sums, which were significantly altered due to increased liver TAG content in wild-type carriers (first diagram) or in I148M PNPLA3 carriers (second diagram). Positive ratios denote lipids, which are higher in subjects with high liver TAG content compared to normal TAG group, whereas negative ratios denote lipids, which are lower in subjects with high liver TAG content compared to normal TAG group. For DAGs, both fatty acid chains were determined (see as DAG(XX:X/YY:Y). First numbers denote the length of fatty acid chain and second number after ":" denote the number of double bounds. DAG(FAXX:X) depict the sum of DAGs with the denoted fatty acid chain (FA). Bold lipids depict DAG(FA18:1) lipid species, which are significantly increased in wild-type PNPLA3 carriers, but remained unchanged in I148M PNPLA3 carriers. TAG: liver triacylglycerol content; wt: wild-type allele with I148I; var: I148M variants, which encode I148M (heterozygous) or M148M (homozygous) variants, respectively. ns: non-significant differences.

\section{Discussion and Conclusions}

The I148M PNPLA3 variant is the best characterized and most influential determinant of NAFLD [19]. Patients with this PNPLA3 variant are also characterized with higher prevalence of NASH and hepatocellular carcinoma [19], however, they show normal insulin sensitivity [8,14]. 
The underlying mechanism of the dissociation between high liver fat and normal insulin sensitivity remains obscure.

To study the key lipid species, which are known to be involved in hepatic insulin resistance [21], we performed an unbiased lipidomics analysis from subjects with high and normal liver fat content with wild-type or I148M PNPLA3 variants. Our data showed, that DAG (FA18:1) lipid species were elevated in the liver of wild-type carriers upon increased liver fat content, however, these lipids remained unaltered in subjects, who carry I148M PNPLA3 variant. When the enzyme activity of wild-type PNPLA3 was characterized, PNPLA3 (as a lipase) showed hydrolytic activity against mono(MAG), di- and triacylglycerols [16]. Interestingly, Huang et al. also observed that the wild-type PNPLA3 strongly prefers oleic acid (C18:1)-containing lipids as a substrate [16]. The I148M PNPLA3 variant was not studied for substrate preference, but it showed diminished hydrolytic activity against MAG, DAG, and TAG. Since PNPLA3 owns substrate specificity against C18:1 containing lipids and the I148M variant show diminished TAG hydrolytic activity, it is conceivable that in patients carrying I148M variant, liver TAG(FA18:1) species could be recognized by PNPLA3 in a lower extent than in wild-type carriers, hindering accumulation of DAG (FA18:1). On the other hand, PNPLA3 is not the only lipase metabolizing TAGs and some TAG(FA18:1) lipid species showed lower, but some others showed higher levels in the I148M PNPLA3 variants upon increased liver TAG content when compared to wild-type carriers (Supplementary Table S1).

Rodent studies suggested that PNPLA3 deficiency is associated with reduced hepatic DAG (FA18:1) content. High sucrose diet fed PNPLA3 knock-out mice showed only a decreased DAG (34:1) lipid content (probably consisting of DAG (C16:0/C18:1)), however, all other lipid species (phosphatidic acid, lysophosphatidic acid, TAG or other DAGs) remained unchanged compared to wild-type controls [17]. Knock-down of PNPLA3 with antisense oligonucleotides in high fat diet fed rats resulted in ameliorated hepatic steatosis, which was associated with lower total DAG, DAG (C16:0/C18:1) and DAG (C18:1/C18:1) lipid species [32]. The authors suggested that the lower levels of these DAGs led to reduction of membrane localized (activated) protein kinase $\mathrm{C}$ epsilon $(\mathrm{PKC} \varepsilon)$ level, which could not interfere with insulin signaling and this mechanism was postulated to be the reason for the improved hepatic insulin sensitivity found in these PNPLA3 knock-down animals [32]. Jelenik et al. demonstrated that mice with hepatic insulin resistance showed elevated hepatic content of DAG (C16:0/C18:1) and DAG (C18:1/C18:1) lipid species, which was associated with higher PKC $\varepsilon$ activation and reduced tyrosine phosphorylation of insulin receptor substrate 2 (IRS2), which is a hallmark of impaired insulin signaling [22]. Furthermore, there are several studies [33], which reported an elevated DAG (FA18:1) content in skeletal muscle of insulin resistant patients with obesity [34] and type 2 diabetes [35]. The authors claimed that the elevated DAG levels could activate PKC theta (PKC $)$ in skeletal muscle, which in turn leads to impaired insulin signaling causing insulin resistance [35]. Moreover, elevated DAG species were also found in the liver of subjects, who showed hepatic insulin resistance [23]. From all DAG species, hepatic cytosolic level of DAG (C16:0/C18:1) and DAG (C18:1/C18:1) were two out of the three most abundant DAGs, which showed the strongest negative correlation with suppression of endogenous glucose production (EGP) [23]. Impairment in the suppression of EGP is a sign for hepatic insulin resistance [36,37]. These results indicate that elevation of DAG (FA18:1) lipid species is a characteristic of insulin resistance and impairment in PNPLA3 function is associated with lower content of hepatic DAG (FA18:1). Whether DAG (FA18:1) is attributed to specific functions in comparison with other DAG species is not clarified yet, but it is possible. Dziewulska et al. reported that mice fed with triolein diet (TAG (C18:1/C18:1/C18:1))

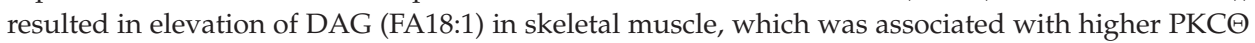
activation but lower serine phosphorylation of protein kinase B and diminished glucose transporter 4 translocation [38], which are signs for insulin resistance [39]. The authors also observed, that tristearin $\operatorname{diet}$ (TAG (C18:0/C18:0/C18:0)) feeding did not exert the former effects [38]. These results suggest that DAG (FA18:1) possibly owns a specific function among DAGs and it could serve as a strong activator of PKCs (PKC $\varepsilon$ in the liver and PKC $\Theta$ in the muscle), which, in turn, could diminish insulin signaling. 
The PNPLA3 variant cohorts consisted of mainly heterozygous (I148M) and only one homozygous (M148M) carrier in each group. Previous studies also combined hetero- and homozygous carriers of the PNPLA3 variant and did not report differences in lipid composition or insulin sensitivity $[8,10,15,40]$ suggesting that these variants are comparable. We, therefore, also analyzed all carriers of the PNPLA3 I148M/M148M genotype together in this study. The limitations of this study are the comorbidities of the subjects and the small study size, however, surgical samples were necessary to obtain sufficient tissue for the lipid analyses. Since we did not have detailed information on alcohol intake of the patients, it cannot be ruled out that some of the patients had an alcohol related cause of fatty liver rather than NAFLD. Furthermore, liver samples fulfilling the criteria of the defined groups were very limited. Therefore, further sub-analyses exceeding the initially selected groups are not possible in this study.

We found that fatty liver in subjects carrying wild-type PNPLA3 is associated with elevated hepatic DAG (FA18:1) content. These DAG (FA18:1) species were shown to disturb insulin signaling in the liver $[22,23]$. However, hepatic DAG (FA18:1) species remained unaltered in subjects carrying I148M PNPLA3 allele with fatty liver. Therefore, we hypothesize that I148M PNPLA3 carriers may be protected from insulin resistance via the unaltered content of DAG (FA18:1) species due to impaired PNPLA3 TAG lipase activity (Figure 3).

A

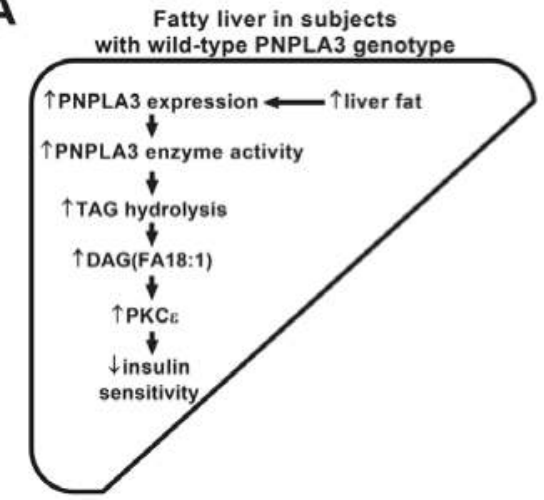

B

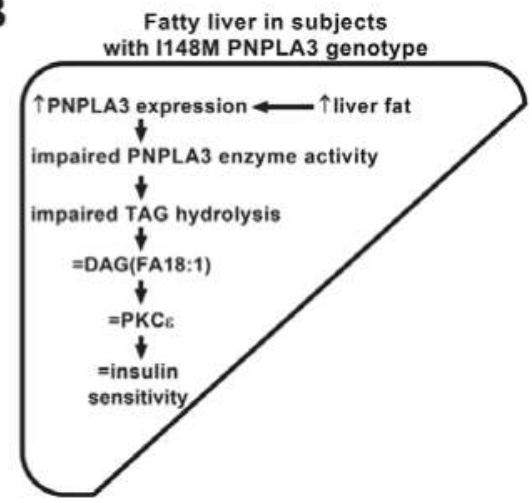

Figure 3. Hypothetical scheme showing the association between I148M PNPLA3 variant and normal insulin sensitivity. Arrows demonstrate higher $(\uparrow)$ or lower $(\downarrow)$ metabolite contents, transcript levels, enzyme activities or insulin sensitivity, respectively. Previous data shown, that liver fat content is positively associated with hepatic mRNA expression of PNPLA3, which was not altered in subjects carrying I148M PNPLA3 variant [10,14,32]. Our lipid data showed that hepatic DAG (FA18:1) species were elevated in fatty liver of wild-type PNPLA3 carriers (A), which was not observed in I148M PNPLA3 carriers (B). Elevated DAG (FA18:1) in the liver was shown to activate protein kinase c epsilon (PKC $\varepsilon$ ), which, in turn, reduces tyrosin phosphorylation of insulin receptor substrate 2 (P-Tyr-IRS2) [22,23], a key molecule transmitting insulin signaling in the liver [39]. Due to the attenuated tyrosin phosphorylation of IRS2, insulin sensitivity could be impaired (as postulated earlier [22,23]) in subjects carrying wild-type PNPLA3 allele, but not in I148M PNPLA3 carriers.

Supplementary Materials: Supplementary materials can be found at http:/ /www.mdpi.com/2072-6643/10/9/ $1314 /$ s1. Table S1. Lipids and sum of lipids, which are significantly changed due to high vs. normal TAG level in subjects.

Author Contributions: Conceptualization: S.L., A.P., H.-U.H.; methodology: D.M., C.P.; formal analysis: A.F., M.K., M.H. (Miriam Hoene); resources: B.A.J., M.H. (Martin Heni), A.K.; writing-original draft: A.F.; writing-review and editing: D.M., M.K., M.H. (Miriam Hoene), B.A.J., M.H. (Martin Heni), A.K., C.P., S.L., H.-U.H., and A.P. 
Funding: This work was supported by a grant from the German Federal Ministry of Education and Research (BMBF) to the German Center for Diabetes Research (DZD e.V.).

Acknowledgments: We gratefully acknowledge Ines Wagener, Andreas Vosseler, Anja Dessecker, and Ellen Kollmar for their assistance in the clinical studies (Department of Internal Medicine IV, Division of Endocrinology, Diabetology, Angiology, Nephrology, and Clinical Chemistry, University Hospital Tübingen, Tübingen, Germany).

Conflicts of Interest: The authors declare no conflict of interest. D.M. and C.P. are employed by Sciex Germany GmbH, Darmstadt, Germany, which, however, had no influence in the design of the study; analyses, or interpretation of data; in the writing of the manuscript; or in the decision to publish the results.

$\begin{array}{ll}\text { Abbreviations } \\ \text { DAG } & \text { Diacylglycerol } \\ \text { FA } & \text { Fatty acid } \\ \text { group 1 } & \text { Subjects with normal liver fat and wild-type PNPLA3 } \\ \text { group 2 } & \text { Subjects with normal liver fat and I148M/M148M PNPLA3 variants } \\ \text { group 3 } & \text { Subjects with high TAG and wild-type PNPLA3 } \\ \text { group 4 } & \text { Subjects with high TAG and I148M/M148M PNPLA3 variants } \\ \text { MAG } & \text { Monoacylglycerol } \\ \text { NAFLD } & \text { Nonalcoholic fatty liver disease } \\ \text { NASH } & \text { Nonalcoholic steatohepatitis } \\ \text { PKC } & \text { Protein kinase C } \\ \text { PNPLA3 } & \text { Patatin-like phospholipase domain-containing protein 3 } \\ \text { TAG } & \text { Triacylglycerol } \\ \text { var } & \text { I148M/M148M PNPLA3 variants } \\ \text { wt } & \text { Wild-type PNPLA3 allele }\end{array}$

\section{References}

1. Stefan, N.; Schick, F.; Häring, H.U. Causes, characteristics, and consequences of metabolically unhealthy normal weight in humans. Cell Metab. 2017, 26, 292-300. [CrossRef] [PubMed]

2. Stefan, N.; Häring, H.U. The role of hepatokines in metabolism. Nat. Rev. Endocrinol. 2013, 9, 144-152. [CrossRef] [PubMed]

3. Younossi, Z.; Anstee, Q.M.; Marietti, M.; Hardy, T.; Henry, L.; Eslam, M.; George, J.; Bugianesi, E. Global burden of NAFLD and NASH: Trends, predictions, risk factors and prevention. Nat. Rev. Gastroenterol. Hepatol. 2018, 15, 11-20. [CrossRef] [PubMed]

4. Younossi, Z.M.; Loomba, R.; Rinella, M.E.; Bugianesi, E.; Marchesini, G.; Neuschwander-Tetri, B.A.; Serfaty, L.; Negro, F.; Caldwell, S.H.; Ratziu, V.; et al. Current and future therapeutic regimens for nonalcoholic fatty liver disease and nonalcoholic steatohepatitis. Hepatology 2018, 68, 361-371. [CrossRef] [PubMed]

5. Böhm, A.; Hoffmann, C.; Irmler, M.; Schneeweiss, P.; Schnauder, G.; Sailer, C.; Schmid, V.; Hudemann, J.; Machann, J.; Schick, F.; et al. TGF-beta contributes to impaired exercise response by suppression of mitochondrial key regulators in skeletal muscle. Diabetes 2016, 65, 2849-2861. [CrossRef] [PubMed]

6. Franko, A.; Neschen, S.; Rozman, J.; Rathkolb, B.; Aichler, M.; Feuchtinger, A.; Brachthäuser, L.; Neff, F.; Kovarova, M.; Wolf, E.; et al. Bezafibrate ameliorates diabetes via reduced steatosis and improved hepatic insulin sensitivity in diabetic TallyHo mice. Mol. Metab. 2017, 6, 256-266. [CrossRef] [PubMed]

7. Goto, T.; Itoh, M.; Suganami, T.; Kanai, S.; Shirakawa, I.; Sakai, T.; Asakawa, M.; Yoneyama, T.; Kai, T.; Ogawa, Y. Obeticholic acid protects against hepatocyte death and liver fibrosis in a murine model of nonalcoholic steatohepatitis. Sci. Rep. 2018, 8, 8157. [CrossRef] [PubMed]

8. Romeo, S.; Kozlitina, J.; Xing, C.; Pertsemlidis, A.; Cox, D.; Pennacchio, L.A.; Boerwinkle, E.; Cohen, J.C.; Hobbs, H.H. Genetic variation in PNPLA3 confers susceptibility to nonalcoholic fatty liver disease. Nat. Genet. 2008, 40, 1461-1465. [CrossRef] [PubMed]

9. Baclig, M.O.; Lozano-Kuhne, J.P.; Mapua, C.A.; Gopez-Cervantes, J.; Natividad, F.F. Genetic variation I148M in patatin-like phospholipase 3 gene and risk of non-alcoholic fatty liver disease among Filipinos. Int. J. Clin. Exp. Med. 2014, 7, 2129-2136. [PubMed] 
10. Kantartzis, K.; Peter, A.; Machicao, F.; Machann, J.; Wagner, S.; Konigsrainer, I.; Konigsrainer, A.; Schick, F.; Fritsche, A.; Häring, H.U.; et al. Dissociation between fatty liver and insulin resistance in humans carrying a variant of the patatin-like phospholipase 3 gene. Diabetes 2009, 58, 2616-2623. [CrossRef] [PubMed]

11. Stojkovic, I.A.; Ericson, U.; Rukh, G.; Riddestrale, M.; Romeo, S.; Orho-Melander, M. The PNPLA3 Ile148Met interacts with overweight and dietary intakes on fasting triglyceride levels. Genes Nutr. 2014, 9, 388. [CrossRef] [PubMed]

12. Sevastianova, K.; Santos, A.; Kotronen, A.; Hakkarainen, A.; Makkonen, J.; Silander, K.; Peltonen, M.; Romeo, S.; Lundbom, J.; Lundbom, N.; et al. Effect of short-term carbohydrate overfeeding and long-term weight loss on liver fat in overweight humans. Am. J. Clin. Nutr. 2012, 96, 727-734. [CrossRef] [PubMed]

13. Scorletti, E.; West, A.L.; Bhatia, L.; Hoile, S.P.; McCormick, K.G.; Burdge, G.C.; Lillycrop, K.A.; Clough, G.F.; Calder, P.C.; Byrne, C.D. Treating liver fat and serum triglyceride levels in NAFLD, effects of PNPLA3 and TM6SF2 genotypes: Results from the WELCOME trial. J. Hepatol. 2015, 63, 1476-1483. [CrossRef] [PubMed]

14. Peter, A.; Kovarova, M.; Nadalin, S.; Cermak, T.; Konigsrainer, A.; Machicao, F.; Stefan, N.; Häring, H.U.; Schleicher, E. PNPLA3 variant I148M is associated with altered hepatic lipid composition in humans. Diabetologia 2014, 57, 2103-2107. [CrossRef] [PubMed]

15. Luukkonen, P.K.; Zhou, Y.; Sadevirta, S.; Leivonen, M.; Arola, J.; Oresic, M.; Hyotylainen, T.; Yki-Jarvinen, H. Hepatic ceramides dissociate steatosis and insulin resistance in patients with non-alcoholic fatty liver disease. J. Hepatol. 2016, 64, 1167-1175. [CrossRef] [PubMed]

16. Huang, Y.; Cohen, J.C.; Hobbs, H.H. Expression and characterization of a PNPLA3 protein isoform (I148M) associated with nonalcoholic fatty liver disease. J. Biol. Chem. 2011, 286, 37085-37093. [CrossRef] [PubMed]

17. Kumari, M.; Schoiswohl, G.; Chitraju, C.; Paar, M.; Cornaciu, I.; Rangrez, A.Y.; Wongsiriroj, N.; Nagy, H.M.; Ivanova, P.T.; Scott, S.A.; et al. Adiponutrin functions as a nutritionally regulated lysophosphatidic acid acyltransferase. Cell Metab. 2012, 15, 691-702. [CrossRef] [PubMed]

18. Mancina, R.M.; Matikainen, N.; Maglio, C.; Soderlund, S.; Lundbom, N.; Hakkarainen, A.; Rametta, R.; Mozzi, E.; Fargion, S.; Valenti, L.; et al. Paradoxical dissociation between hepatic fat content and de novo lipogenesis due to PNPLA3 sequence variant. J. Clin. Endocrinol. Metab. 2015, 100, 821-825.

19. Petaja, E.M.; Yki-Jarvinen, H. Definitions of normal liver fat and the association of insulin sensitivity with acquired and genetic NAFLD-A systematic review. Int. J. Mol. Sci. 2016, 17, 633. [CrossRef] [PubMed]

20. Li, J.Z.; Huang, Y.; Karaman, R.; Ivanova, P.T.; Brown, H.A.; Roddy, T.; Castro-Perez, J.; Cohen, J.C.; Hobbs, H.H. Chronic overexpression of PNPLA3I148M in mouse liver causes hepatic steatosis. J. Clin. Investig. 2012, 122, 4130-4144. [CrossRef] [PubMed]

21. Samuel, V.T.; Shulman, G.I. Nonalcoholic fatty liver disease as a nexus of metabolic and hepatic diseases. Cell Metab. 2018, 27, 22-41. [CrossRef] [PubMed]

22. Jelenik, T.; Kaul, K.; Sequaris, G.; Flogel, U.; Phielix, E.; Kotzka, J.; Knebel, B.; Fahlbusch, P.; Horbelt, T.; Lehr, S.; et al. Mechanisms of insulin resistance in primary and secondary nonalcoholic fatty liver. Diabetes 2017, 66, 2241-2253. [CrossRef] [PubMed]

23. Ter Horst, K.W.; Gilijamse, P.W.; Versteeg, R.I.; Ackermans, M.T.; Nederveen, A.J.; la Fleur, S.E.; Romijn, J.A.; Nieuwdorp, M.; Zhang, D.; Samuel, V.T.; et al. Hepatic diacylglycerol-associated protein kinase cepsilon translocation links hepatic steatosis to hepatic insulin resistance in humans. Cell Rep. 2017, 19, 1997-2004. [CrossRef] [PubMed]

24. Ollero, M. Methods for the study of lipid metabolites in cystic fibrosis. J. Cyst. Fibros. 2004, 3, 97-98. [CrossRef] [PubMed]

25. Lehmann, R.; Franken, H.; Dammeier, S.; Rosenbaum, L.; Kantartzis, K.; Peter, A.; Zell, A.; Adam, P.; Li, J.; $\mathrm{Xu}, \mathrm{G}$; ; et al. Circulating lysophosphatidylcholines are markers of a metabolically benign nonalcoholic fatty liver. Diabetes Care 2013, 36, 2331-2338. [CrossRef] [PubMed]

26. Franko, A.; Huypens, P.; Neschen, S.; Irmler, M.; Rozman, J.; Rathkolb, B.; Neff, F.; Prehn, C.; Dubois, G.; Baumann, M.; et al. Bezafibrate improves insulin sensitivity and metabolic flexibility in STZ-induced diabetic mice. Diabetes 2016, 65, 2540-2552. [CrossRef] [PubMed]

27. Markgraf, D.F.; Al-Hasani, H.; Lehr, S. Lipidomics-reshaping the analysis and perception of type 2 diabetes. Int. J. Mol. Sci. 2016, 17, 1841. [CrossRef] [PubMed]

28. Ubhi, B.K. Direct Infusion-Tandem Mass Spectrometry (DI-MS/MS) analysis of complex lipids in human plasma and serum using the lipidyzer platform. Methods Mol. Biol. 2018, 1730, 227-236. [PubMed] 
29. Peter, A.; Kantartzis, K.; Machicao, F.; Machann, J.; Wagner, S.; Templin, S.; Königsrainer, I.; Königsrainer, A.; Schick, F.; Fritsche, A.; et al. Visceral obesity modulates the impact of apolipoprotein C3 gene variants on liver fat content. Int. J. Obes. 2012, 36, 774-782. [CrossRef] [PubMed]

30. Franko, A.; Kovarova, M.; Feil, S.; Feil, R.; Wagner, R.; Heni, M.; Köngisrainer, A.; Ruoß, M.; Nüssler, A.K.; Weigert, C.; et al. cGMP-dependent protein kinase I (cGKI) modulates human hepatic stellate cell activation. Metabolism 2018, 88, 22-30. [CrossRef] [PubMed]

31. Chen, S.; Hoene, M.; Li, J.; Li, Y.; Zhao, X.; Häring, H.U.; Schleicher, E.D.; Weigert, C.; Xu, G.; Lehmann, R. Simultaneous extraction of metabolome and lipidome with methyl tert-butyl ether from a single small tissue sample for ultra-high performance liquid chromatography/mass spectrometry. J. Chromatogr. A 2013, 1298, 9-16. [CrossRef] [PubMed]

32. Kumashiro, N.; Yoshimura, T.; Cantley, J.L.; Majumdar, S.K.; Guebre-Egziabher, F.; Kursawe, R.; Vatner, D.F.; Fat, I.; Kahn, M.; Erion, D.M.; et al. Role of patatin-like phospholipase domain-containing 3 on lipid-induced hepatic steatosis and insulin resistance in rats. Hepatology 2013, 57, 1763-1772. [CrossRef] [PubMed]

33. Amati, F. Revisiting the diacylglycerol-induced insulin resistance hypothesis. Obes. Rev. 2012, 13, 40-50. [CrossRef] [PubMed]

34. Moro, C.; Galgani, J.E.; Luu, L.; Pasarica, M.; Mairal, A.; Bajpeyi, S.; Schmitz, G.; Langin, D.; Liebisch, G.; Smith, S.R. Influence of gender, obesity, and muscle lipase activity on intramyocellular lipids in sedentary individuals. J. Clin. Endocrinol. Metab. 2009, 94, 3440-3447. [CrossRef] [PubMed]

35. Szendroedi, J.; Yoshimura, T.; Phielix, E.; Koliaki, C.; Marcucci, M.; Zhang, D.; Jelenik, T.; Muller, J.; Herder, C.; Nowotny, P.; et al. Role of diacylglycerol activation of PKCtheta in lipid-induced muscle insulin resistance in humans. Proc. Natl. Acad. Sci. USA 2014, 111, 9597-9602. [CrossRef] [PubMed]

36. Franko, A.; von Kleist-Retzow, J.C.; Neschen, S.; Wu, M.; Schommers, P.; Böse, M.; Kunze, A.; Hartmann, U.; Sanchez-Lasheras, C.; Stoehr, O.; et al. Liver adapts mitochondrial function to insulin resistant and diabetic states in mice. J. Hepatol. 2014, 60, 816-823. [CrossRef] [PubMed]

37. Ayala, J.E.; Bracy, D.P.; McGuinness, O.P.; Wasserman, D.H. Considerations in the design of hyperinsulinemic-euglycemic clamps in the conscious mouse. Diabetes 2006, 55, 390-397. [CrossRef] [PubMed]

38. Dziewulska, A.; Dobrzyn, P.; Jazurek, M.; Pyrkowska, A.; Ntambi, J.M.; Dobrzyn, A. Monounsaturated fatty acids are required for membrane translocation of protein kinase C-theta induced by lipid overload in skeletal muscle. Mol. Membr. Biol. 2012, 29, 309-320. [CrossRef] [PubMed]

39. Boucher, J.; Kleinridders, A.; Kahn, C.R. Insulin receptor signaling in normal and insulin-resistant states. Cold Spring Harb. Perspect. Biol. 2014, 6, a009191. [CrossRef] [PubMed]

40. Hyysalo, J.; Gopalacharyulu, P.; Bian, H.; Hyotylainen, T.; Leivonen, M.; Jaser, N.; Juuti, A.; Honka, M.J.; Nuutila, P.; Olkkonen, V.M.; et al. Circulating triacylglycerol signatures in nonalcoholic fatty liver disease associated with the I148M variant in PNPLA3 and with obesity. Diabetes 2014, 63, 312-322. [CrossRef] [PubMed]

(C) 2018 by the authors. Licensee MDPI, Basel, Switzerland. This article is an open access article distributed under the terms and conditions of the Creative Commons Attribution (CC BY) license (http:/ / creativecommons.org/licenses/by/4.0/). 
Article

\title{
Soybean Oil-Derived Poly-Unsaturated Fatty Acids Enhance Liver Damage in NAFLD Induced by Dietary Cholesterol
}

Janin Henkel 1,*(D), Eugenia Alfine 2,3 Juliana Saín 1,4 (D), Korinna Jöhrens 5 Daniela Weber 6 José P. Castro ${ }^{6,7}$, Jeannette König ${ }^{6}$, Christin Stuhlmann ${ }^{1}$, Madita Vahrenbrink ${ }^{1}$, Wenke Jonas $^{3,8}$, André Kleinridders ${ }^{2,3}$ and Gerhard P. Püschel ${ }^{1}$

1 Department of Nutritional Biochemistry, Institute of Nutritional Science, University of Potsdam, D-14558 Nuthetal, Germany; jsain@fbcb.unl.edu.ar (J.S.); stuhlman@uni-potsdam.de (C.S.); vahrenbrink@uni-potsdam.de (M.V.); gpuesche@uni-potsdam.de (G.P.P.)

2 German Institute of Human Nutrition, Junior Research Group Central Regulation of Metabolism; D-14558 Nuthetal, Germany; Eugenia.Alfine@dife.de (E.A.); Andre.Kleinridders@dife.de (A.K.)

3 German Center for Diabetes Research (DZD), D-85764 München-Neuherberg, Germany; Wenke.Jonas@dife.de

4 Department of Biological Sciences, Food Science and Nutrition, Faculty of Biochemistry and Biological Sciences, National University of the Litoral (UNL), Santa Fe S3000, Argentina

5 Institute of Pathology, Carl Gustav Carus University Hospital Dresden; D-01307 Dresden, Germany; korinna.joehrens@uniklinikum-dresden.de

6 Department of Molecular Toxicology, German Institute of Human Nutrition; D-14558 Nuthetal, Germany; Daniela.Weber@dife.de (D.W.); Jose.Castro@dife.de (J.P.C.); Jeannette.Koenig@dife.de (J.K.)

7 Department of Medicine, Division of Genetics, Brigham and Women's Hospital and Harvard Medical School, Boston, MA 02115, USA

8 Department of Experimental Diabetology, German Institute of Human Nutrition; D-14558 Nuthetal, Germany

* Correspondence: jhenkel@uni-potsdam.de; Tel.: +49-33200-88-5285

Received: 31 August 2018; Accepted: 17 September 2018; Published: 18 September 2018

\begin{abstract}
While the impact of dietary cholesterol on the progression of atherosclerosis has probably been overestimated, increasing evidence suggests that dietary cholesterol might favor the transition from blunt steatosis to non-alcoholic steatohepatitis (NASH), especially in combination with high fat diets. It is poorly understood how cholesterol alone or in combination with other dietary lipid components contributes to the development of lipotoxicity. The current study demonstrated that liver damage caused by dietary cholesterol in mice was strongly enhanced by a high fat diet containing soybean oil-derived w6-poly-unsaturated fatty acids (w6-PUFA), but not by a lard-based high fat diet containing mainly saturated fatty acids. In contrast to the lard-based diet the soybean oil-based diet augmented cholesterol accumulation in hepatocytes, presumably by impairing cholesterol-eliminating pathways. The soybean oil-based diet enhanced cholesterol-induced mitochondrial damage and amplified the ensuing oxidative stress, probably by peroxidation of poly-unsaturated fatty acids. This resulted in hepatocyte death, recruitment of inflammatory cells, and fibrosis, and caused a transition from steatosis to NASH, doubling the NASH activity score. Thus, the recommendation to reduce cholesterol intake, in particular in diets rich in w6-PUFA, although not necessary to reduce the risk of atherosclerosis, might be sensible for patients suffering from non-alcoholic fatty liver disease.
\end{abstract}

Keywords: NASH; non-alcoholic fatty liver disease (NAFLD); cholesterol; PUFA; inflammation; oxidative stress 


\section{Introduction}

The poor reputation of dietary cholesterol traces back to its supposed promoting role in the development of atherosclerosis [1]. However, the impact of dietary cholesterol on atherosclerosis has apparently been largely overestimated [2,3] and more recent recommendations for cardio-protective diets do not include the previously suggested radical reduction of cholesterol intake [4]. Although from the point of view of atherosclerosis, dietary cholesterol might be less relevant than previously assumed, it has reentered the focus of interest because of its potential role in the progression of non-alcoholic fatty liver disease (NAFLD) $[5,6]$.

NAFLD describes a range of liver pathologies that have in common a lipid accumulation in hepatocytes in the absence of significant alcohol intake. NAFLD is a major and growing health problem [7]. While in recent years the prevalence of NAFLD in the general population has attained a level of $25 \%$, it is present in the vast majority of overweight or obese patients and is considered the hepatic manifestation of the metabolic syndrome [8]. The disease pattern ranges from fully reversible blunt steatosis (NAFL) to a chronically progressive disease (non-alcoholic steatohepatitis, NASH) that is characterized by varying degrees of hepatocyte death, inflammation, and fibrosis and may ultimately result in liver cirrhosis, hepatocellular carcinoma, and terminal organ failure [9]. The transition from steatosis to the more severe forms of the disease occurs in roughly one third of affected patients, but it is still unclear what triggers this progression. However, there is evidence that dietary cholesterol might impact this transition. A number of independent animal experimental studies showed that enrichment of a high fat diet with 0.2 to $2 \%$ cholesterol resulted in a rapid progression from blunt steatosis to a NASH-like phenotype with ballooning, infiltration with inflammatory cells, and fibrosis [10-14]. Similarly, animal experiments [15] and clinical studies [16] using the cholesterol uptake inhibitor ezitimibe suggest that inhibition of cholesterol uptake from the gut might protect against NASH development. The mechanisms of how dietary cholesterol might trigger the transition to NASH have so far not been fully elucidated. The supposed mechanisms include activation of ER-stress response in hepatocytes, impairment of mitochondrial function resulting in oxidative stress, and the activation of resident or infiltrating macrophages by danger-associated molecular patterns (DAMPs) released from cholesterol-laden dying hepatocytes $[17,18]$.

Our current study supports the view that dietary cholesterol can trigger NASH-development by causing mitochondrial dysfunction and oxidative stress and shows that these patho-mechanisms are severely aggravated by the presence of poly-unsaturated fatty acids (PUFA) in dietary fat.

\section{Materials and Methods}

All chemicals were of analytical or higher grade and obtained from local providers unless otherwise stated.

Animals and experimental design. Male C57BL/6JRj mice (own breeding) were housed in type II-cages at $20 \pm 2{ }^{\circ} \mathrm{C}$ with a $12 \mathrm{~h}$ light/dark-cycle. Mice were randomly assigned to one of the following diet groups with free access to food and drinking water for 20 weeks: standard chow diet (V153 R/M-H; Ssniff, Soest, Germany) (STD), 0.75\% cholesterol on a standard diet (CHO + STD), $0.75 \%$ cholesterol in a high fat diet containing w6-PUFA-rich soybean oil (CHO + SOY; Altromin, Lage, Germany) or $0.75 \%$ cholesterol in a high fat diet containing mainly lard as fat source (CHO + LAR, D12451; Research Diets, New Brunswick, NJ, USA). Detailed diet composition is shown in Table 1. Mice had access to wooden gnawing sticks to avoid excessive teeth growth. Body weight was measured weekly. Mice were killed by cervical dislocation after isoflurane anesthesia. Serum and organs were snap-frozen in liquid nitrogen and stored at $-70{ }^{\circ} \mathrm{C}$ for biochemical analysis, aliquots of the organs were fixed for histological examination. Animal experiments were performed according to the ARRIVE guidelines. Treatment of the animals followed the German animal protection laws and was performed with approval of the state animal welfare committee (LUGV Brandenburg, V3 2347). 
Table 1. Diet composition. Mice diets used in the feeding experiment. Standard chow diet (STD), $0.75 \%$ cholesterol in a Standard diet $(\mathrm{CHO}+\mathrm{CHO}), 0.75 \%$ cholesterol in a high fat diet containing $\omega 6$-PUFA-rich soybean oil ( $\mathrm{CHO}+\mathrm{SOY}), 0.75 \%$ cholesterol in a high fat diet containing mainly lard as fat source $(\mathrm{CHO}+\mathrm{LAR})$.

\begin{tabular}{ccccc}
\hline & STD & CHO + STD & CHO + SOY & CHO + LAR \\
\hline Metabolizing energy (kcal/g) & 3.06 & 3.06 & 4.64 & 4.73 \\
Energy from carbohydrates (\%) & 65 & 65 & 35 & 35 \\
Energy from protein (\%) & 25 & 25 & 16 & 20 \\
Energy from fat (\%) & 10 & 10 & 49 & 45 \\
$\quad$ Cholesterol (\%) & 0.00 & 0.75 & 0.75 & 0.75 \\
Fatty acid composition & & & & \\
Saturated fatty acids (g/100g) & 0.55 & 0.55 & 4.00 & 7.26 \\
Mono-unsaturated fatty acids (g/100g) & 0.64 & 0.64 & 5.75 & 8.69 \\
Poly-unsaturated fatty acids (g/100g) & 2.01 & 2.01 & 14.50 & 6.36 \\
\hline
\end{tabular}

In vivo experiments. Body fat content was measured at the beginning and at the end of the diet intervention by nuclear magnetic resonance spectroscopy (EchoMRI 2012 Body Composition Analyzer, Houston, TX, USA). The oral glucose tolerance test was performed in week 18 after an overnight fast by oral gavage of glucose ( $2 \mathrm{mg} / \mathrm{kg}$ body weight). Glucose and insulin levels were measured at the times indicated by a glucose sensor (Breeze2, Bayer; Berlin, Germany) or an insulin ELISA kit (Crystall Chem; Downers Grove, IL, USA).

Serum and tissue analysis. Serum parameters were quantified by an automated analyzer (Cobas Mira S, Hoffmann-La Roche, Basel, Switzerland) with the appropriate commercially available reagent kits. Liver triglycerides were determined by TRIGS-assay (Randox; Crumlin, UK). Total and free cholesterol in liver tissue was determined by a modified version of a protocol described previously [19]. Briefly, frozen tissues were homogenized by sonication using phosphate buffer $(10 \mathrm{mM}, \mathrm{pH} 7.4)$ containing $1 \%$ polyoxyehylen-10-tridecylether. Homogenates were heated (5 min at $70{ }^{\circ} \mathrm{C}$ ) to inactivate enzymes and then centrifuged for $10 \mathrm{~min}$ at $4{ }^{\circ} \mathrm{C}$. Aliquots of supernatant were incubated in the presence or absence of $0.5 \mathrm{U} / \mathrm{mL}$ of cholesterol esterase to quantify the total and free cholesterol, respectively. The reaction buffer contained $100 \mathrm{mmol} / \mathrm{L}$ Tris (pH 7.7); $6 \mathrm{mmol} / \mathrm{l}$ phenol, $1 \mathrm{mmol} / \mathrm{L}$ 4-aminoantipyrine, $4 \mathrm{mmol} / \mathrm{L}$ 3,4-dichlorophenol, $10 \mathrm{mmol} / \mathrm{L}$ sodium cholate, $3 \mathrm{~g} / \mathrm{L}$ fatty alcohol polyglycol ether, $50 \mathrm{mmol} / \mathrm{L} \mathrm{MgCl} 2,0.2 \mathrm{U} / \mathrm{mL}$ cholesterol oxidase, and $0.4 \mathrm{U} / \mathrm{mL}$ peroxidase. The quinoneimine dye formed after $30 \mathrm{~min}$ is proportional to the quantity of cholesterol and was detected at $500 \mathrm{~nm}$. A calibration curve was performed using a cholesterol solution $200 \mathrm{mg} \%$ (m/v) in fatty alcohol polyglycol ether $(3 \mathrm{~g} / \mathrm{L})$. The esterified cholesterol was quantified by the difference between total and free cholesterol. Malondialdehyde was quantified by HPLC with fluorescence detection as described previously [11].

Histology. Formalin-fixed and paraffin-embedded liver sections $(2-3 \mu \mathrm{m})$ were stained with Hematoxylin \& Eosin or Sirius Red (both Sigma-Aldrich, Taufkirchen, Germany). Immunohistochemistry analyses were performed with anti-F4/80 antibody (AbD Serotec, Bio-Rad, Munich, Germany). Terminal deoxynucleotidyl transferase dUTP Nick End Labeling (TUNEL) assay was achieved with the Click-iT ${ }^{\text {TM }}$ TUNEL Colorimetric IHC Detection Kit (Thermo Fisher Scientific, Berlin, Germany). Histological steatosis, inflammation and fibrosis were graded according to the NASH activity score (NAS) $[20,21]$ by a liver pathologist (KJ) blinded to the diet. Quantification of histological staining of Sirius Red, F4/80, and TUNEL-positive cells was performed by using ImageJ software (version ImageJ 1.51j8, Wayne Rasband, National Institutes of Health, USA) in images of five randomly chosen fields of each liver. Details are described in the Methods section of the Supplementary Material.

Real-time RT-PCR analysis. RNA isolation, reverse transcription, and qPCR were performed as previously described [22]. Results are expressed as relative gene expression normalized to expression levels of reference genes (Hprt, Eef2 and Srsf4) according to the formula: fold induction = 
$2^{(a-b)}$ gene of interest $/ 2(a-b)$ reference genes. Parameter "a" is the arithmetic mean of all Ct-values from samples of the STD group and parameter " $\mathrm{b}$ " is the Ct-value of every single sample. For calculations with more than one reference gene the geometric mean of the difference $(a-b)$ of each reference gene was used.

Western blot and Oxyblot analysis. Western blot was performed as described previously [23] with anti-PGC-1 $\alpha$ antibody and oxidative phosphorylation cocktail for Western blot (both abcam, Cambridge, UK), as well as Ponceau S-staining (Sigma-Aldrich, Taufkirchen, Germany) as a loading control. Oxyblot analysis was done as described [11] with anti-DNP antibody (Sigma-Aldrich, Taufkirchen, Germany). Visualization of immune complexes was performed by using a chemoluminescence reagent in the ChemiDoc ${ }^{\mathrm{TM}}$ Imaging System with ImageLab software (Bio-Rad, Munich, Germany).

Statistical analysis. The statistical significance of differences was determined by one-way-ANOVA with Tukey's post hoc test for multiple comparisons or Krukal-Wallis test for non-parametric samples as detailed in the legends to the figures using GraphPad Prism version 6 for Windows (GraphPad Software, La Jolla, California, CA, USA). Differences with a $p \leq 0.05$ were considered statistically significant.

\section{Results}

\subsection{Diet-Induced Weight Gain, Insulin Resistance and NAFLD}

Mice received either standard chow diet (STD), chow diet enriched with $0.75 \%$ cholesterol (CHO + STD), a soybean oil-based high fat diet with $0.75 \%$ cholesterol $(\mathrm{CHO}+\mathrm{SOY})$ or a lard-based high fat diet with $0.75 \%$ cholesterol $(\mathrm{CHO}+\mathrm{LAR})$ for 20 weeks, as described in Table 1 . Animals on both high fat diets gained more weight than animals fed either chow diet or cholesterol-enriched chow diet (Figure 1A). The high fat diet-induced weight gain could be attributed to an increase in fat mass (Figure 1B) while the fat-free mass remained largely unaltered. Despite similar weight gain and increase in fat mass, animals fed the $\mathrm{CHO}+\mathrm{LAR}$ diet were significantly more insulin resistant than animals receiving $\mathrm{CHO}+\mathrm{SOY}$ diet (Figure $1 \mathrm{C}$ ). As expected from the body weight data, $\mathrm{CHO}+$ STD-fed animals showed no signs of insulin resistance.

Serum cholesterol levels increased only slightly $(20 \%)$ in animals receiving the $\mathrm{CHO}+$ STD diet (Figure 2A). By contrast, serum cholesterol concentrations were doubled in comparison to the control in animals receiving either one of the high fat diets with cholesterol. Notably, no difference in serum cholesterol levels was observed between $\mathrm{CHO}+\mathrm{SOY}$ - and CHO + LAR-fed animals (Figure 2A).

Unexpectedly, but in keeping with data of many independent studies in the literature [11,24,25], serum triglyceride levels were not elevated but instead were decreased in animals receiving either one of the cholesterol-enriched diets, irrespective of their fat content (Figure 2B). Total cholesterol was increased in livers of all animals receiving cholesterol-enriched diets. However, whereas CHO + STD and $\mathrm{CHO}+$ LAR-fed animals showed a similar approximately 2 to 3-fold increase in hepatic cholesterol content, animals receiving $\mathrm{CHO}+\mathrm{SOY}$ diet exhibited a 6-fold increase in hepatic total cholesterol content (Figure 2C). Notably, free cholesterol was not significantly increased in CHO + STD-fed or $\mathrm{CHO}+$ LAR-fed animals in comparison to STD-fed animals, whereas free cholesterol content was doubled in $\mathrm{CHO}+\mathrm{SOY}$-fed mice (Figure 2C). In line with this, high amounts of cholesterol crystals could be detected only in livers of $\mathrm{CHO}+\mathrm{SOY}$-fed mice whereas only few or no cholesterol crystals were visible in livers of $\mathrm{CHO}+\mathrm{STD}$ or $\mathrm{CHO}+\mathrm{LAR}$-fed mice (own observation). 

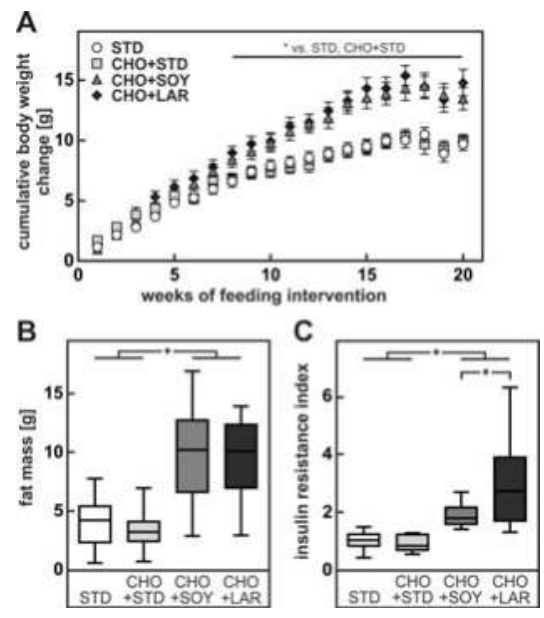

Figure 1. Increase in body weight, fat mass and insulin resistance in mice fed a $\mathrm{CHO}+\mathrm{SOY}$ or $\mathrm{CHO}+\mathrm{LAR}$ diet for 20 weeks. (A) Cumulative body weight change. (B) Fat mass in week 20. (C) Insulin resistance index was calculated by the sum of the products of insulin concentration $\times$ glucose concentration during the oral glucose tolerance test. Values are median (line), upper- and lower quartile (box) and extremes (whiskers) of 17-35 (A,B) or 8-10 (C) mice per group. Statistics: Multiple Student's $t$-test for unpaired samples (A) or one-way-ANOVA with Tukey's post hoc test for multiple comparisons $(\mathbf{B}, \mathbf{C}) .{ }^{*} p<0.05$.
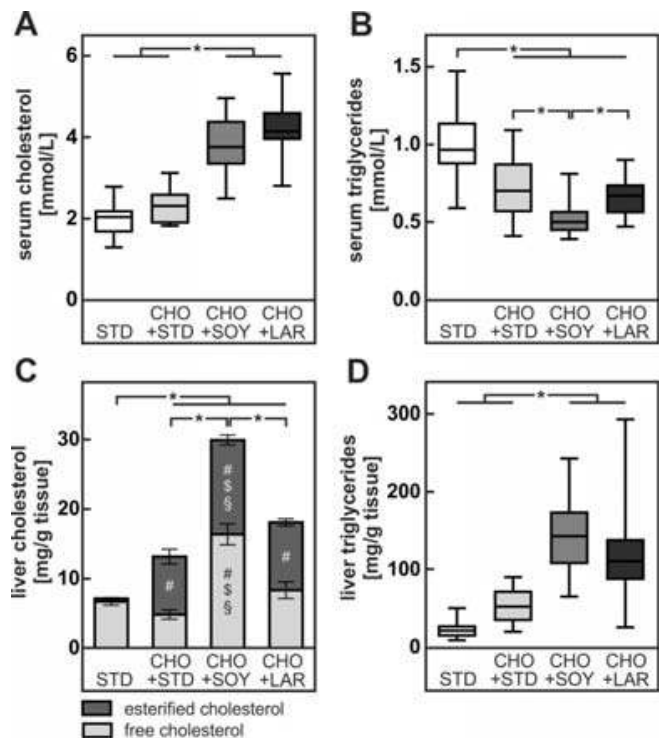

Figure 2. Diet-induced changes in serum and liver lipids after 20 weeks. (A) Cholesterol concentrations in serum. (B) Triglyceride concentrations in serum. (C) Levels of free and esterified cholesterol in liver. (D) Triglyceride levels in liver. Values are median (line), upper- and lower quartile (box) and extremes (whiskers) (A,B,D) or mean and sem (C) of 17-35 mice per group. Statistics: One-way-ANOVA with Tukey's post hoc test for multiple comparisons. ${ }^{*}: p<0.05$. Separate statistic for free and esterified cholesterol (C): \#: vs. STD, \$: vs. CHO + STD, §: vs. CHO+ LAD with $p<0.05$. 
Although weight gain was unaltered in animals receiving $\mathrm{CHO}+\mathrm{STD}$ diet, these animals had a pronounced hepatic steatosis (see below, Figure 4). Hepatic triglyceride content increased more than twofold in comparison to chow-fed animals (Figure 2D). Hepatic triglyceride accumulation was more pronounced in animals receiving cholesterol-enriched high fat diets. Livers of $\mathrm{CHO}+\mathrm{SOY}$-fed and CHO + LAR-fed animals contained 7-fold or 5-fold more triglycerides than STD-fed animals, respectively (Figure 2D). The difference between the two high fat diets was, however, not significant.

In summary, mice fed a CHO + SOY diet accumulated significantly higher amounts of free and esterified cholesterol in the liver compared to mice fed one of the other cholesterol-containing diets. Since the $\mathrm{CHO}+\mathrm{STD}, \mathrm{CHO}+\mathrm{SOY}$ and $\mathrm{CHO}+\mathrm{LAD}$ diets contained equal amounts of cholesterol, the combination of dietary cholesterol and w6-PUFA-rich soybean oil may favor hepatic cholesterol accumulation.

The more pronounced increase in hepatic cholesterol content can either be the consequence of an enhanced uptake or a diminished excretion or conversion of cholesterol. In accordance with the latter hypothesis, the expression of the cholesterol export pump Abcg5 was induced more than fourfold in animals receiving either $\mathrm{CHO}+\mathrm{STD}$ or $\mathrm{CHO}+\mathrm{LAR}$ diet (Figure 3A). By contrast, the export pump was induced merely twofold, and hence significantly less, in animals receiving $\mathrm{CHO}$ + SOY diet than in either of the other two cholesterol containing diets (Figure 3A). Gene expression of Abcg8, the heterodimerization partner of Abcg5, was similar, yet it did not reach significance (Figure 3B). In comparison to the standard chow diet, Abca1, another cholesterol transporter mainly expressed in macrophages, was induced approximately 1.32-fold in livers of mice fed any of the cholesterol-containing diets. The increase was significant only in the $\mathrm{CHO}+\mathrm{STD}$ diet group and no significant differences between the cholesterol-fed groups were observed.
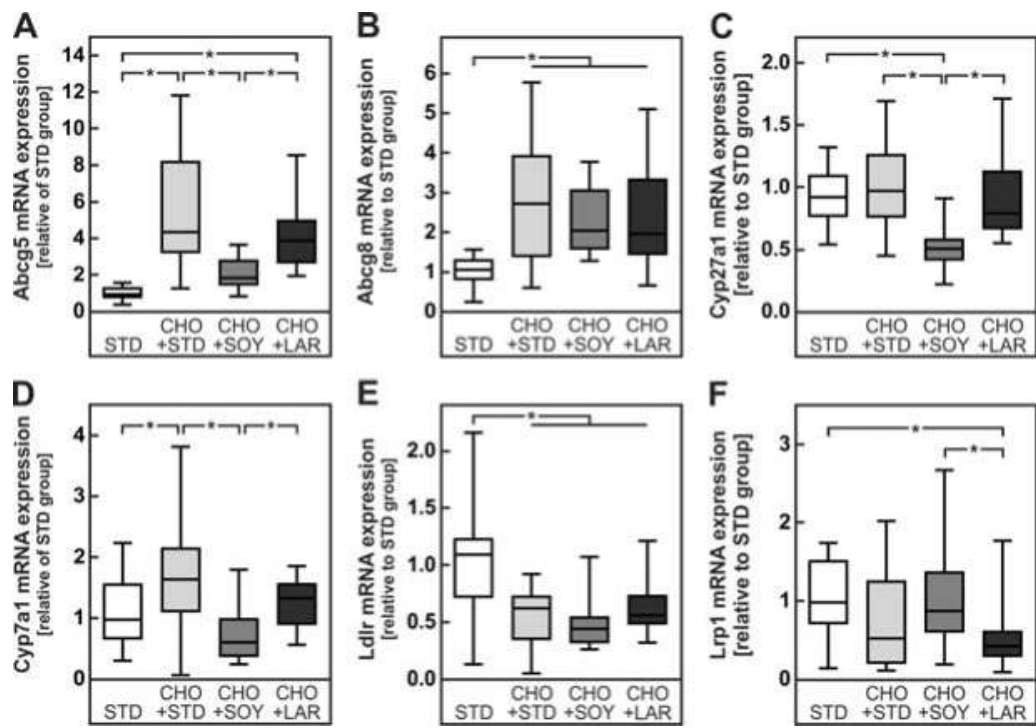

Figure 3. Markers of cholesterol metabolism in mice fed a cholesterol-containing diet for 20 weeks. Relative mRNA expression of the cholesterol transporters ATP-binding cassette sub-family G (Abcg) member 5 (A) and 8 (B), the cholesterol- metabolizing enzymes cytochrome P450 family 27 a1 (Cyp27a1, C) and family 7 a1 (Cyp7a1, D) as well as the transporters for the cholesterol intake LDL receptor (Ldlr, E) and LDL receptor related protein 1 (Lrp1, F) in mice liver. Values are median (line), upper- and lower quartile (box) and extremes (whiskers) of 17-35 mice per group. Statistics: One-way-ANOVA with Tukey's post hoc test for multiple comparisons. *: $p<0.05$. 
In addition, the expression of Cyp27a1, a key enzyme for the conversion of cholesterol into bile acids, was significantly repressed in livers of CHO + SOY-fed mice (Figure 3C). Similarly, Cyp7a1 was repressed in $\mathrm{CHO}+\mathrm{SOY}$-fed animals, whereas it was unaffected or even induced in $\mathrm{CHO}+\mathrm{LAR}$ diet and $\mathrm{CHO}+$ STD diet-fed animals, respectively (Figure 3D). Gene expression of the LDL receptor was repressed to a similar extent in livers of animals receiving any of the three cholesterol-containing diets (Figure 3E). By contrast, the expression of the LDL receptor related protein 1 (Lrp1) was slightly or significantly reduced in livers of animals receiving the $\mathrm{CHO}+\mathrm{STD}$ or $\mathrm{CHO}+\mathrm{LAR}$ diets, whereas expression was unaltered in livers of $\mathrm{CHO}+\mathrm{SOY}$-fed animals (Figure 3F).

In conclusion, the enhanced cholesterol accumulation in livers of $\mathrm{CHO}+\mathrm{SOY}$-fed mice can be explained by a decreased Abcg5-mediated cholesterol export, reduced Cyp27a1-dependent conversion of cholesterol into bile acids, as well as impaired repression of Lrp1-related cholesterol uptake into hepatocytes.

Following, livers were examined histologically to determine the NASH activity score (NAS) (Table 2 and Figure 4). No signs of NAFLD were detected in livers of STD-fed control animals. By contrast, all animals receiving cholesterol-enriched diets had a positive NAS. However, while the average NAS for CHO + STD-fed and CHO + LAR-fed animals reached a maximum of 4 and hence indicated the presence of blunt steatosis, the average NAS of $\mathrm{CHO}+\mathrm{SOY}$-fed mice was above 7, clearly indicating the presence of active NASH (Table 2).

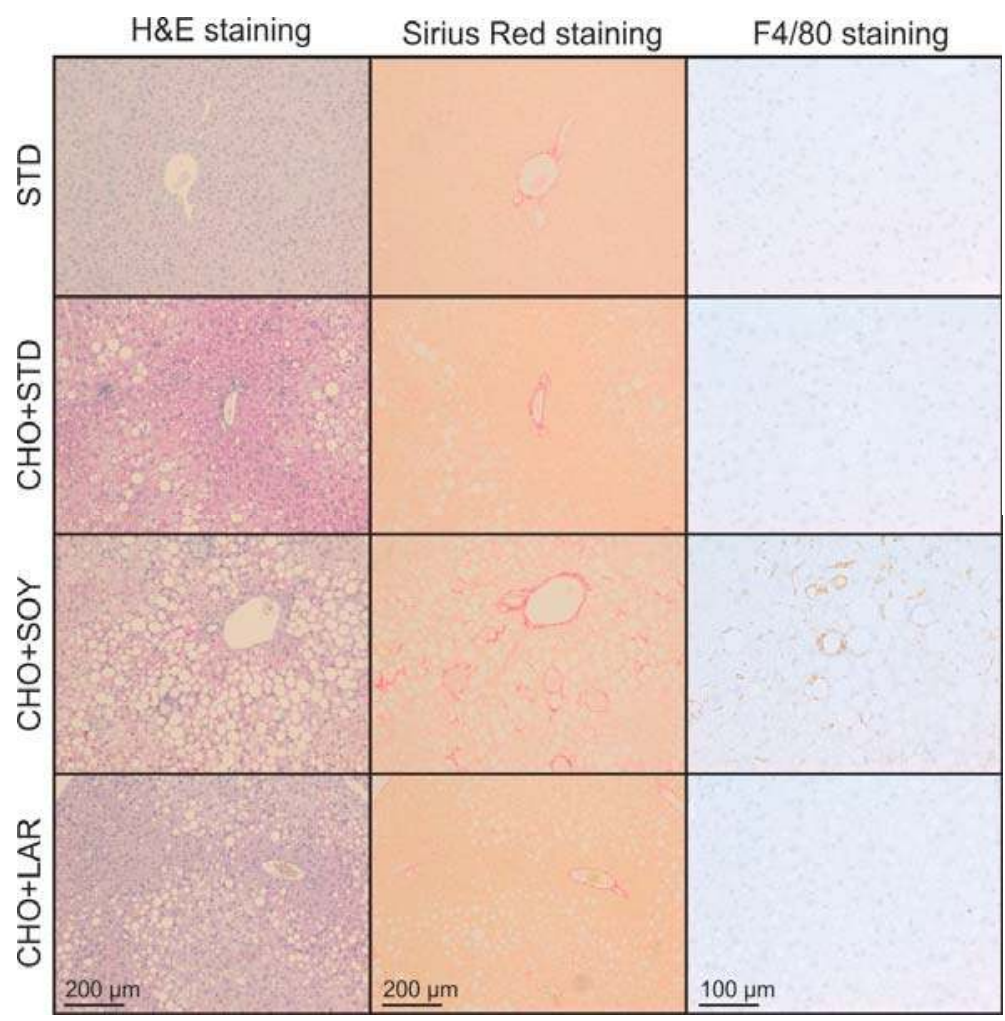

Figure 4. $\mathrm{CHO}+\mathrm{SOY}$ diet induced steatohepatitis with steatosis, fibrosis, and macrophage infiltration. Mice received the diets for 20 weeks. Representative microphotographs of liver sections, magnification $10 \times$ or $20 \times$. 
Table 2. NASH activity score grading steatosis, ballooning (hepatocyte hypertrophy), inflammation, and fibrosis. Values are mean \pm SEM of 17-35 mice per group. Statistics: Kruskal-Wallis test with Dunn's post hoc test for multiple comparisons. \#: vs. STD, \$: vs. CHO + STD, §: vs. CHO + LAD with $p<0.05$.

\begin{tabular}{ccccc}
\hline Scoring Parameter & STD & CHO + STD & CHO + SOY & CHO + LAR \\
\hline Steatosis & $0.00 \pm 0.00$ & $1.53 \pm 0.37(\#)$ & $3.77 \pm 0.08(\#, \$, \S)$ & $2.41 \pm 0.33(\#)$ \\
Hepatocyte hypertrophy & $0.00 \pm 0.00$ & $0.35 \pm 0.15$ & $1.73 \pm 0.10(\#, \$, \S)$ & $0.76 \pm 0.18(\#)$ \\
Inflammation & $0.10 \pm 0.05$ & $0.53 \pm 0.15$ & $1.50 \pm 0.13(\#, \$, \S)$ & $0.41 \pm 0.12$ \\
Fibrosis & $0.26 \pm 0.08$ & $0.59 \pm 0.12$ & $0.80 \pm 0.07(\#)$ & $0.47 \pm 0.12$ \\
NASH activity score (NAS) & $0.36 \pm 0.10$ & $3.00 \pm 0.59(\#)$ & $7.80 \pm 0.20(\#, \$, \S)$ & $4.06 \pm 0.49(\#)$ \\
\hline
\end{tabular}

\subsection{Diet-Induced Inflammation and Fibrosis}

All cholesterol-containing diets apparently triggered an inflammatory response in the liver. However, in accordance with the higher NAS, animals receiving the $\mathrm{CHO}+\mathrm{SOY}$ diet showed more pronounced signs of inflammation (Figure 4 right panel, quantification in Figure 5B). The expression of the chemotactic cytokine $\mathrm{Ccl} 2(\mathrm{Mcp}-1)$ was increased about two-fold in animals receiving $\mathrm{CHO}+$ STD or CHO + LAR diets, whereas an almost 10-fold increase was observed in CHO + SOY diet-fed animals (Figure 5A). Consequently, the expression of the macrophage markers F4/80, Cd68, and Cd11b was increased by all cholesterol-containing diets, but was significantly higher in $\mathrm{CHO}+\mathrm{SOY}$-fed animals than in animals receiving any of the other diets (Figure 5B-D). Similarly, the induction of the pro-inflammatory cytokine TNF- $\alpha$ was two-fold higher in livers of $\mathrm{CHO}+\mathrm{SOY}$-fed mice than in animals that received the $\mathrm{CHO}+\mathrm{STD}$ or $\mathrm{CHO}+\mathrm{LAR}$ diet (Figure 5E). Inducible nitric oxide synthase (Nos2, iNos), a key enzyme in inflammation-dependent NO production was only induced in livers of $\mathrm{CHO}+\mathrm{SOY}$-fed animals (Figure 5F). Furthermore, a significantly higher amount of TUNEL-positive hepatocytes were detected in livers of $\mathrm{CHO}+\mathrm{SOY}$-fed mice compared to mice fed any of the other diets, showing increased hepatic apoptosis (Figure $5 \mathrm{G}$ ).

In order to assess fibrosis, liver slices were stained with Sirius Red (Figure 4). A significant increase in fibrosis was only observed in animals receiving $\mathrm{CHO}+\mathrm{SOY}$ diet, whereas all other animals only showed minor age-appropriate positive staining for Sirius Red (Figure 5H). In accordance with these histological data, collagen 1a1 expression was slightly induced in animals fed CHO + STD or $\mathrm{CHO}+\mathrm{LAR}$ diets, whereas a more than 10 -fold induction was observed in livers of $\mathrm{CHO}+\mathrm{SOY}$-fed mice (Figure 5I).

These results show that only mice fed a $\mathrm{CHO}+\mathrm{SOY}$ diet developed clear signs of hepatic inflammation with macrophage infiltration and increased expression of pro-inflammatory cytokines as well as hepatocyte apoptosis and liver fibrosis. 
A
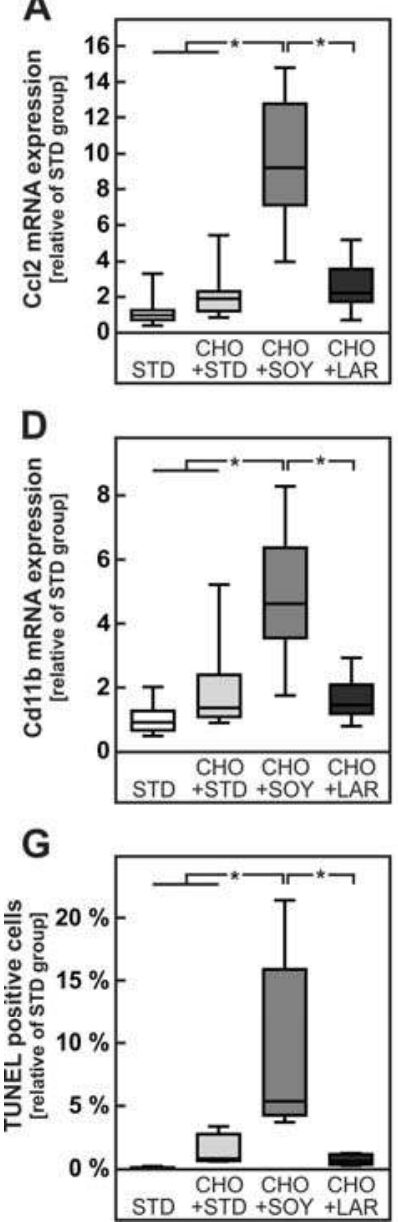

B

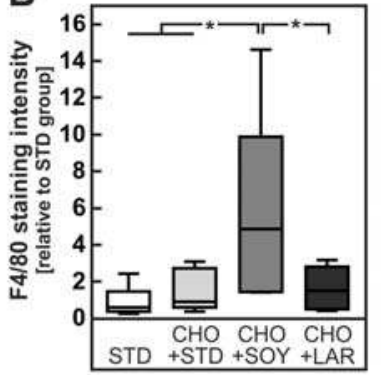

E
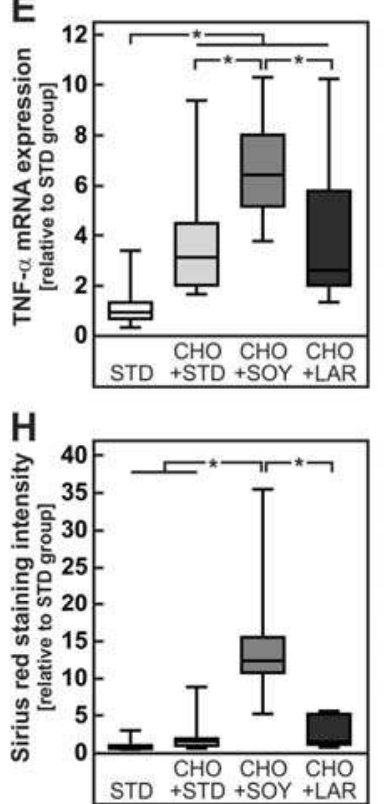

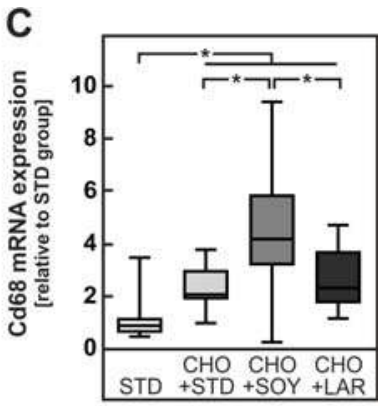

$F$
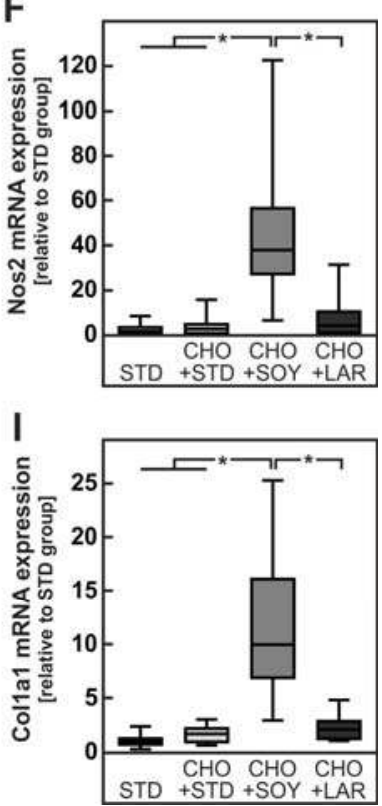

Figure 5. Enhanced macrophage infiltration, inflammation, apoptosis and fibrosis in mice fed a $\mathrm{CHO}$ + SOY diet. Mice received the diets for 20 weeks. (A) Relative mRNA expression of the chemokine $\mathrm{Ccl} 2$ (alternative name Mcp-1) in mice liver. (B) Quantification of F4/80-stained microphotographs of the liver. $(\mathbf{C}, \mathbf{D}, \mathbf{E}, \mathbf{F})$ Relative mRNA expression of the macrophage markers Cd68 (C) and Cd11b (D, gene name Itgam), the cytokine tumor necrosis factor $\alpha$ (TNF- $\alpha, \mathbf{E})$ and the enzyme inducible nitric oxide synthase 2 (F, alternative name iNos) in mice liver. (G) Quantification of hepatocyte apoptosis by TUNEL assay. (H) Quantification of Sirius Red-stained microphotographs of the liver calculated by dense intensity of Sirius Red relative to the amount of cytosolic background per field in 5 randomly chosen microphotographs per liver section. (I) Relative mRNA expression of the fibrosis marker collagen 1a1 (Col1a1) in mice liver. Values are median (line), upper- and lower quartile (box) and extremes (whiskers) of 17-35 (A,C,D,E,F,I), 4-7 (B,G) or 13-16 (H) mice per group. Statistics: One-way-ANOVA with Tukey's post hoc test for multiple comparisons. *: $p<0.05$.

\subsection{Diet-Induced Mitochondrial Damage and Oxidative Stress}

The cholesterol-dependent induction of liver damage has previously been attributed to mitochondrial damage resulting from cholesterol accumulation in mitochondrial membranes. However, judging from the expression of complex I, II, and IV of the respiratory chain, dietary cholesterol alone apparently did cause low but no severe mitochondrial damage in our model 
(Figure 6A). A mild reduction of complex I, II, and IV content was also observed in livers from animals receiving $\mathrm{CHO}+\mathrm{LAR}$ diet. In stark contrast, complex I, II, and IV proteins were dramatically reduced in livers of $\mathrm{CHO}+\mathrm{SOY}$-fed animals, indicating severe mitochondrial damage in only this group (Figure 6A). In keeping with these data, the amount of PGC- $1 \alpha$ protein was strongly reduced in livers of animals receiving the $\mathrm{CHO}+\mathrm{SOY}$ diet (Figure $6 \mathrm{~B}$ ).
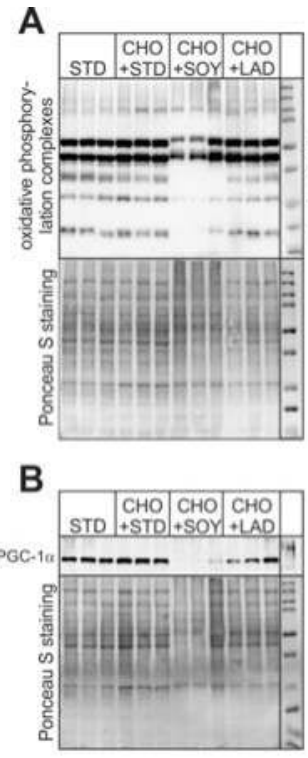

D

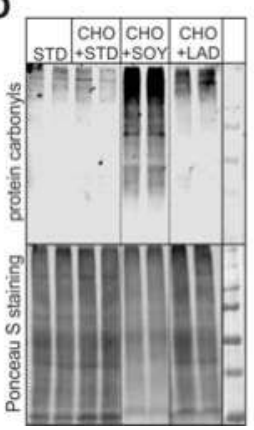

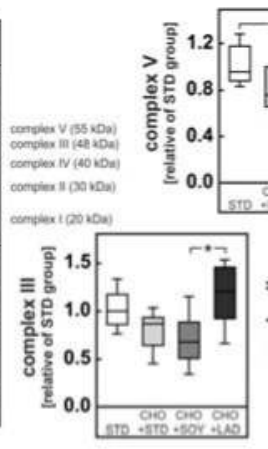
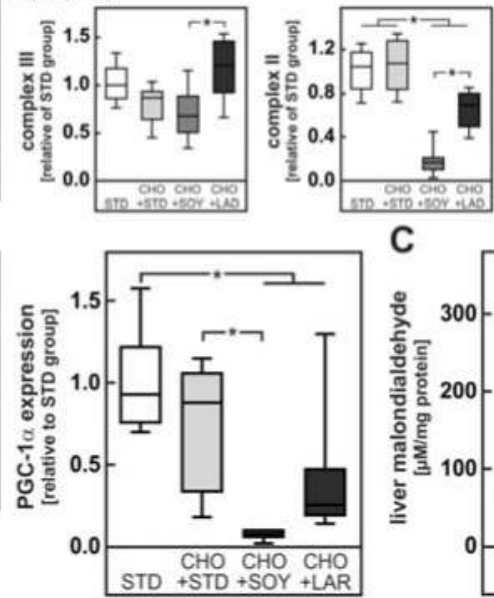

C

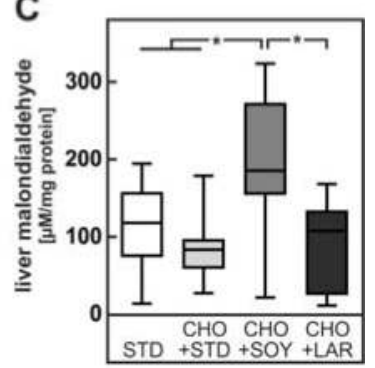

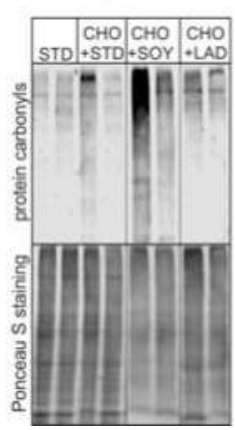

Figure 6. Increased mitochondrial damage and oxidative stress in mice fed a $\mathrm{CHO}+\mathrm{SOY}$ diet for 20 weeks. (A,B) Hepatic protein expression of the oxidative phosphorylation complexes (A) and PGC-1 $\alpha(B)$. Dense intensity was normalized to Ponceau S staining, which was verified on the same Western blot membrane as a loading control and calculated relative to the STD group in each gel. A representative blot is shown. All original blots are provided in the Supplementary Material, Figures S1 and S2. (C) Concentration of malondialdehyde in liver as a marker of lipid peroxidation. (D) Determination of protein carbonyls in liver homogenates verified by oxyblot with Ponceau staining as a loading control. Blots were cut at the dotted lines. Original blots are shown in the Supplementary Material, Figure S3. Values are median (line), upper- and lower quartile (box) and extremes (whiskers) of 8-10 (A,B) or 9-28 (C) mice per group. Statistics: One-way-ANOVA with Tukey's post hoc test for multiple comparisons. ${ }^{*}: p<0.05$. 
Impairment of mitochondrial respiration causes severe oxidative stress. Accordingly, levels of malondialdehyde, a reaction product of lipid peroxidation of unsaturated fatty acids, and protein carbonyls were only increased in livers of $\mathrm{CHO}+\mathrm{SOY}$-fed mice (Figure 6C,D).

Thus, cholesterol-induced mitochondrial damage and oxidative stress was clearly enhanced by soybean oil-derived PUFA probably due to augmented lipid peroxidation.

\subsection{Oxidative Stress Preceding Inflammation}

In order to elucidate whether oxidative stress in livers of $\mathrm{CHO}+\mathrm{SOY}$-fed animals was the cause or consequence of the inflammation observed in the livers of these animals, the $\mathrm{CHO}+\mathrm{SOY}$ diet was fed for a shorter period, i.e., 19 days. While liver steatosis, a reduction of PGC-1 $\alpha$, a reduction of complexes of the respiratory chain as well as signs of oxidative stress were already present after 19 days of feeding, no increase in inflammatory markers was detectable, indicating that mitochondrial damage and oxidative stress precede the development of inflammation (Figure 7, Figure S4 in Supplementary Material).

A
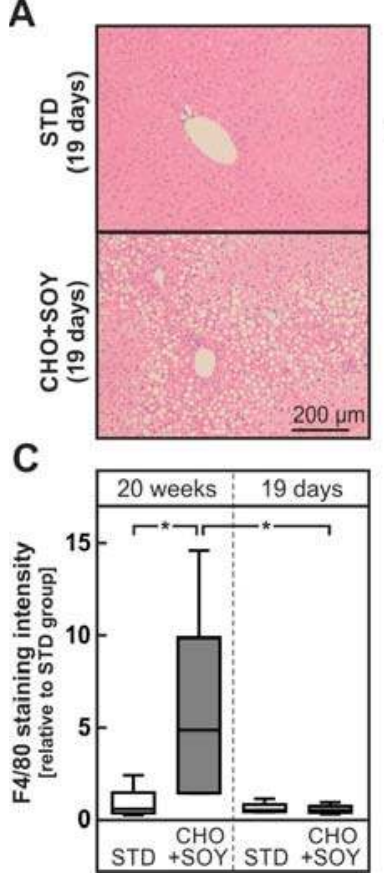

B

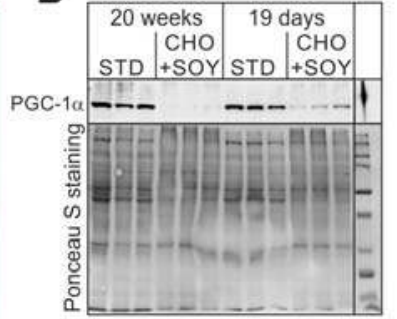

D

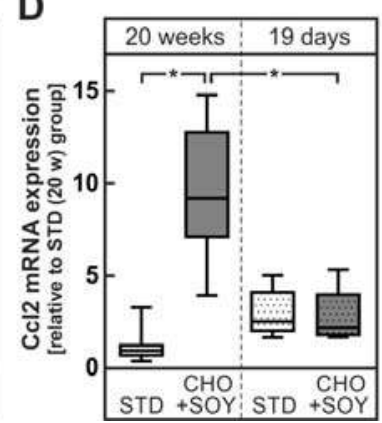

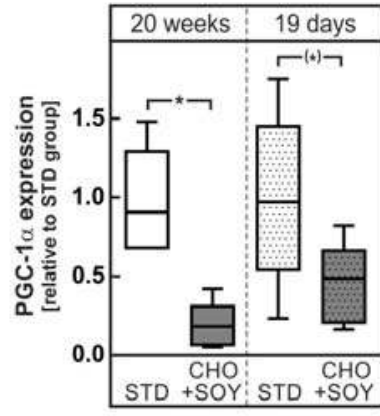

E

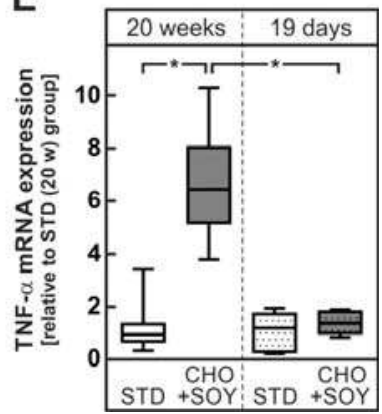

Figure 7. Enhanced mitochondrial damage but no signs of inflammation in mice fed a CHO + SOY diet for 19 days. Mice received the STD CHO + SOY diet for 20 weeks or 19 days. (A) Representative H\&E-stained microphotographs of the liver. (B) Hepatic protein expression of PGC-1 $\alpha$ after 20 week and 19 days feeding intervention. Dense intensity was normalized to Ponceau S staining, which was verified on the same Western blot membrane as a loading control and calculated relative to the STD group in each gel. A representative blot is shown. All original blots are provided in the Supplementary Material, Figure S4. (C) Quantification of F4/80-stained microphotographs of the liver calculated by dense intensity of F4/80 relative to the amount of cytosolic background per field in 5 randomly chosen microphotographs per liver section. (D,E) Relative mRNA expression of Ccl2 (D, alternative name Mcp-1) and tumor necrosis factor $\alpha$ (TNF- $\alpha, E)$ in mice liver. Values are median (line), upperand lower quartile (box) and extremes (whiskers) of $6(\mathrm{~B}, \mathrm{C})$ or $6-7$ (D,E) mice per group. Statistics: One-way-ANOVA with Tukey's post hoc test for multiple comparisons. *: $p<0.05$. 


\section{Discussion}

In our current study we showed that dietary cholesterol induced hepatic steatosis and NAFLD independent of the accompanying lipid content of the diet. However, histological signs of progression to NASH, hepatocyte apoptosis, infiltration with inflammatory cells, and fibrosis only developed when dietary cholesterol was combined with a soybean oil-based high fat diet rich in w6-PUFA. Our results indicate the contribution of two potentially cooperating mechanisms to this transition: (1) soybean oil-derived PUFA increased hepatic cholesterol accumulation, most likely by repression of pathways that are responsible for the elimination of cholesterol via the bile. (2) soybean oil-derived PUFA augmented cholesterol-induced mitochondrial damage and oxidative stress presumably via lipid peroxidation.

\subsection{PUFA-Dependent Increase in Cholesterol Accumulation}

An increase in hepatic cholesterol accumulation by a soybean oil-based high fat diet has been observed previously in a study that compared the tissue distribution of cholesterol in rats fed a soybean oil-based or tallow-based high fat diet [26]. Yet, the underlying mechanisms were not analyzed in this early study. Hepatic cholesterol accumulation in the presence of dietary PUFA could result either from increased hepatic uptake or decreased removal.

Since dietary cholesterol is absorbed via the chylomicron pathway, an increase in hepatic cholesterol uptake should most likely be mediated by an augmented hepatic clearance of remnant particles or absorption of HDL cholesterol. Both processes involve the LDL receptor, the LDL-receptor-related protein 1, and the scavenger receptor class B type1. While the LDL receptor, as expected, was downregulated in animals fed any of the cholesterol-containing diets, gene expression of the LDL receptor related protein 1 (Lrp1) was reduced only in animals receiving $\mathrm{CHO}+\mathrm{STD}$ or $\mathrm{CHO}$ + LAR diets, but not in the CHO + SOY-fed animals (Figure 3E,F). These differences were however rather small and unlikely to account for the two-fold increase in hepatic cholesterol observed in $\mathrm{CHO}$ + SOY-fed animals (Figure 2C). In the literature, a PUFA-induced increase in SR-B1 expression was described in a genetically obese rat strain [27] that was supposed to result in a more efficient hepatic uptake of cholesterol from HDL and hence might contribute to hepatic cholesterol accumulation and at the same time contribute to the anti-atherogenic effect of dietary PUFA.

The current data indicate that a dietary PUFA-dependent inhibition of cholesterol removal from the liver either as free cholesterol or after conversion into bile acids is the more likely explanation for the pronounced cholesterol accumulation in livers of $\mathrm{CHO}+\mathrm{SOY}$-fed animals. Cholesterol can be excreted directly into the bile by the ABCG5/ABCG8 export pump [28]. The expression of Abcg5 was induced four- to sixfold in $\mathrm{CHO}+\mathrm{LAR}-$ or $\mathrm{CHO}+\mathrm{STD}-\mathrm{fed}$ animals, respectively. By contrast a significantly lower twofold induction was observed in livers of CHO + SOY-fed animals (Figure 3A,B). Similarly, the expression of the genes for enzymes involved in bile acid formation was repressed by $\mathrm{CHO}+\mathrm{SOY}$ diet feeding. The CHO + SOY diet is rich in w6-PUFA whereas the content in $\omega 3$-fatty acids is relatively low. In contrast to $\omega 6$-PUFA, $\omega 3$-PUFA appeared to increase the expression both of cholesterol export pumps and key enzymes of bile acid synthesis [29]. Interestingly, whereas $\omega 6$-fatty acids favor accumulation of cholesterol in the liver, long chain $\omega 3$-poly-unsaturated fatty acids seem to counteract this effect. Thus, hamsters fed a diet that contained 38\% linoleic acid (18:2(w6)) accumulated almost twice as much cholesterol ester in the liver as animals fed a similar diet in which half of the linoleic acid was replaced by long chain $\omega 3$-fatty acids, mainly eicosapentaenoic acid (20:5( $\omega 3))$ and docosahexaenoic acid (22:6(w3)) [30]. Similarly, hepatic cholesterol content was reduced by long chain w3-PUFA supplementation in mice fed a high fat high cholesterol diet rich in $\omega 6$-fatty acids [31]. This might in part explain why supplementation with $\omega 3$-fatty acids has repeatedly been reported to protect from NAFLD or NASH development [32,33] while depletion of $\omega 3$-PUFA increased hepatic steatosis [34].

Also highly unlikely, it cannot be entirely excluded that apart from the pronounced differences in the fatty acid composition minor differences in the protein composition between the two high fat diets 
also contributed to the different phenotypes observed in particular to hepatic lipid accumulation, since diets extremely rich in protein appear to protect from hepatic steatosis [35].

\subsection{PUFA-Dependent Enhancement of Oxidative Stress}

Dietary cholesterol initially accumulates in the hepatocyte. This results in an increase in cholesterol content in the membranes of different cellular compartments and the subsequent impairment of their function. Thus, it has been shown that an increment in the cholesterol content of the ER membrane may result in the inhibition of the ER calcium pump, a drop in ER calcium concentration, impaired protein folding, and ER stress ([18] and references therein). It has, however, been questioned, whether this mechanism is relevant for NASH development [36]. Similarly, accumulation of cholesterol in the outer phospholipid monolayer of lipid droplets has been assumed to impair lipid turnover and may result in the formation of cholesterol crystals in lipid droplets [12]. Most importantly, however, excessive incorporation of cholesterol in mitochondrial membranes has been shown to impair the function of the $\alpha$-ketoglutarate carrier that is responsible for the import of reduced glutathione from the cytosol into the mitochondrion [37]. As a consequence, the quenching of reactive oxygen species formed in the respiratory chain is impaired and oxidative stress ensues [38]. The oxidative stress further impairs mitochondrial function and may sensitize the hepatocyte to other death-inducing signals. Apparently, this mechanism was further aggravated by the presence of PUFA in the CHO + SOY diet, since only the combination of cholesterol with soybean oil-based high fat diet resulted in a reduction of mitochondrial respiratory chain proteins and PGC-1 $\alpha$, the master regulator of mitochondrial biogenesis (Figure 6A,B), as well as profound oxidative stress leading to the formation of large amounts of protein carbonyls (Figure 6D). Apart from enhancing cholesterol accumulation in the hepatocyte (see above), PUFA might amplify cholesterol-dependent oxidative stress by lipid peroxidation chain reactions resulting, among others, in the formation of malondialdehyde (Figure 6C). Lipid peroxides have been shown to decrease the content of mitochondrial respiratory chain proteins [39] and cause mitochondrial dysfunction. A reduction in the content of mitochondrial respiratory chain proteins in the liver of NASH patients has been described [25]. Lipid peroxidation also favors the non-enzymatic formation of oxysterols. While oxysterols at low concentrations activate liver $\mathrm{X}$ receptor (LXR) and may initiate pathways that protect against consequences of a cholesterol overload, high oxysterol concentrations induce apoptosis by triggering the mitochondrial apoptotic pathway [40] in hepatoma cells or primary rat hepatocytes, in particular if cells were exposed to a combination of oxysterols and fatty acids. In addition, oxysterols at high concentrations appear to contribute to cell death by antagonizing Akt-dependent survival pathways [41]. Oxysterols are elevated in NAFLD patients [42] and may be causative in NASH development [43].

The oxidative stress-induced death of the cholesterol laden hepatocytes might then trigger the subsequent inflammatory response and the initiation of the development of fibrosis. Notably, signs of mitochondrial damage and oxidative stress preceded the development of inflammation (Figure 7). Hepatocyte detritus may be taken up by Kupffer cells and infiltrating macrophages that have been shown to form crown-like structures around dying hepatocytes [44]. Since cholesterol cannot be removed by macrophages, this results in a self-perpetuating chronic inflammation that triggers scar formation and fibrosis (Figure 4). Activation of macrophages and stellate cells by cholesterol or cholesterol crystals [11,12,45] and oxysterols [46] has been shown to promote this process.

\subsection{Possible Clinical Impact}

There is evidence that dietary cholesterol may also favor NASH development in humans [47-49] and the interruption of intestinal cholesterol absorption by ezetimibe has been shown to be beneficial in NAFLD patients in a meta-analysis of several clinical studies [16]. Notably, in one study ezetimibe reduced the NASH activity score without affecting steatosis [50]. A possible interaction between dietary cholesterol and fatty acid composition of the diet apparently was not systematically analyzed in human studies. However, meta-analysis of several clinical studies showed that supplementation 
with long chain w3-PUFA caused a more or less pronounced reduction in the plasma levels of alanine aminotransferase (ALAT), aspartate aminotransferase (ASAT), and $\gamma$-glutamyltransferase (GGT), indicating a reduction of liver damage. However, the actual NASH activity score was not determined [51].

\section{Conclusions}

A direct translation of the results of the current study to a dietary recommendation for humans is beyond any doubt inappropriate. However, the data suggest that the recommendation to replace saturated fat by fat from sources rich in $\omega 6$-PUFA without a simultaneous reduction of cholesterol intake may be sensible from the point of view of protection against cardiovascular diseases and possibly other consequences of the metabolic syndrome, however, may not be advisable from the point of view of NASH development.

Supplementary Materials: The following are available online at http:/ / www.mdpi.com/2072-6643/10/9/1326/ s1, Supplementary Methods: Quantification of immunohistochemistry analysis; Supplementary Figure S1: Expression of oxidative phosphorylation complexes in liver homogenates; Supplementary Figure S2: Expression of PGC- $1 \alpha$ in liver homogenates; Supplementary Figure S3: Detection of protein carbonyls by oxyblot analysis in liver homogenates; Supplementary Figure S4: Increased mitochondrial damage and oxidative stress in mice fed a $\mathrm{CHO}+\mathrm{SOY}$ diet for 19 days.

Author Contributions: Conceptualization, J.H. and G.P.P.; Data curation, J.H.; Formal analysis, J.H.; Funding acquisition, J.H.; Investigation, J.H. and G.P.P.; Methodology, J.H., E.A., J.S., K.J., D.W., J.P.C., J.K., C.S., and M.V.; Resources, W.J. and A.K.; Supervision, G.P.P.; Validation, J.H., E.A., J.S., W.J., and A.K.; Visualization, J.H.; Writing and original draft, J.H. and G.P.P.; Writing, review and editing, A.K.

Funding: This research was funded by the Deutsche Forschungsgemeinschaft, grant HE-7032/1-1 (to J.H.). Additionally this work was supported by the Deutsche Forschungsgemeinschaft grant project KL 2399/4-1 (to A.K.) and by a grant from the German Ministry of Education and Research (BMBF) and the State of Brandenburg (DZD grant 82DZD00302 to A.K.).

Acknowledgments: The outstandingly skillful technical work of Manuela Kuna as well as the technical assistance of Fabian Gellert, Ines Kahnt, Elisabeth Meyer, and Susann Richter is gratefully acknowledged. The excellent proof-reading competence of Brit-Maren Schjeide is highly appreciated. We further acknowledge the support of Deutsche Forschungsgemeinschaft (German Research Foundation) and Open Access Publication Fund of the University of Potsdam.

Conflicts of Interest: The authors declare no conflict of interest.

\section{References}

1. Lee, Y.T.; Laxton, V.; Lin, H.Y.; Chan, Y.W.F.; Fitzgerald-Smith, S.; To, T.L.O.; Yan, B.P.; Liu, T.; Tse, G. Animal models of atherosclerosis. Biomed. Rep. 2017, 6, 259-266. [CrossRef] [PubMed]

2. McNamara, D.J. Dietary cholesterol, heart disease risk and cognitive dissonance. Pro. Nut. Soc. 2014, 73, 161-166. [CrossRef] [PubMed]

3. Berger, S.; Raman, G.; Vishwanathan, R.; Jacques, P.F.; Johnson, E.J. Dietary cholesterol and cardiovascular disease: A systematic review and meta-analysis. Am. J. Clin. Nutr. 2015, 102, 276-294. [CrossRef] [PubMed]

4. Brownawell, A.M.; Falk, M.C. Cholesterol: Where science and public health policy intersect. Nutr. Rev. 2010, 68, 355-364. [CrossRef] [PubMed]

5. Vinué, Á.; Herrero-Cervera, A.; González-Navarro, H. Understanding the impact of dietary cholesterol on chronic metabolic diseases through studies in rodent models. Nutrients 2018, 10, 939. [CrossRef] [PubMed]

6. Jahn, D.; Kircher, S.; Hermanns, H.M.; Geier, A. Animal models of NAFLD from a hepatologist's point of view. BBA-Mol. Basis. Dis. 2018. [CrossRef] [PubMed]

7. Estes, C.; Razavi, H.; Loomba, R.; Younossi, Z.; Sanyal, A.J. Modeling the epidemic of nonalcoholic fatty liver disease demonstrates an exponential increase in burden of disease. Hepatology 2018, 67, 123-133. [CrossRef] [PubMed]

8. Andronescu, C.I.; Purcarea, M.R.; Babes, P.A. Nonalcoholic fatty liver disease: Epidemiology, pathogenesis and therapeutic implications. J. Med. Life 2018, 11, 20-23. [PubMed] 
9. Brunt, E.M.; Wong, V.W.S.; Nobili, V.; Day, C.P.; Sookoian, S.; Maher, J.J.; Bugianesi, E.; Sirlin, C.B.; Neuschwander-Tetri, B.A.; Rinella, M.E. Nonalcoholic fatty liver disease. Nat. Rev. Dis. Primers 2015, 1, 15080. [CrossRef] [PubMed]

10. Subramanian, S.; Goodspeed, L.; Wang, S.; Kim, J.; Zeng, L.; Ioannou, G.N.; Haigh, W.G.; Yeh, M.M.; Kowdley, K.V.; O’Brien, K.D.; et al. Dietary cholesterol exacerbates hepatic steatosis and inflammation in obese LDL receptor-deficient mice. J. Lipid Res. 2011, 52, 1626-1635. [CrossRef] [PubMed]

11. Henkel, J.; Coleman, C.D.; Schraplau, A.; Jöhrens, K.; Weber, D.; Castro, J.P.; Hugo, M.; Schulz, T.J.; Krämer, S.; Schürmann, A.; et al. Induction of steatohepatitis (NASH) with insulin resistance in wildtype B6 mice by a western-type diet containing soybean oil and cholesterol. Mol. Med. 2017, 23, 70. [CrossRef] [PubMed]

12. Ioannou, G.N.; Subramanian, S.; Chait, A.; Haigh, W.G.; Yeh, M.M.; Farrell, G.C.; Lee, S.P.; Savard, C. Cholesterol crystallization within hepatocyte lipid droplets and its role in murine NASH. J. Lipid Res. 2017, 58, 1067-1079. [CrossRef] [PubMed]

13. Buettner, R.; Ascher, M.; Gäbele, E.; Hellerbrand, C.; Kob, R.; Bertsch, T.; Bollheimer, L.C. Olive oil attenuates the cholesterol-induced development of nonalcoholic steatohepatitis despite increased insulin resistance in a rodent model. Horm. Metab. Res. 2013, 45, 795-801. [CrossRef] [PubMed]

14. Hansen, H.H.; Feigh, M.; Veidal, S.S.; Rigbolt, K.T.; Vrang, N.; Fosgerau, K. Mouse models of nonalcoholic steatohepatitis in preclinical drug development. Drug Discov. Today 2017, 22, 1707-1718. [CrossRef] [PubMed]

15. Wang, X.; Ren, Q.; Wu, T.; Guo, Y.; Liang, Y.; Liu, S. Ezetimibe prevents the development of non-alcoholic fatty liver disease induced by high-fat diet in C57BL/6J mice. Mol. Med. Rep. 2014, 10, 2917-2923. [CrossRef] [PubMed]

16. Nakade, Y.; Murotani, K.; Inoue, T.; Kobayashi, Y.; Yamamoto, T.; Ishii, N.; Ohashi, T.; Ito, K.; Fukuzawa, Y.; Yoneda, M. Ezetimibe for the treatment of non-alcoholic fatty liver disease: A meta-analysis. Hepatol. Res. 2017, 47, 1417-1428. [CrossRef] [PubMed]

17. Tirosh, O. Hypoxic signaling and cholesterol lipotoxicity in fatty liver disease progression. Oxid. Med. Cell. Longev. 2018, 7, 1-15. [CrossRef] [PubMed]

18. Ioannou, G.N. The Role of Cholesterol in the Pathogenesis of NASH. Trends Endocrinol. Metab. 2016, 27, 84-95. [CrossRef] [PubMed]

19. Siedel, J.; Rollinger, W.; Röschlau, P.; Ziegenhorn, J.; Bergmeyer, H.U. Total cholesterol, end-point and kinetic method. In Methods of Enzymatic Analysis; Verlag Chemie GmbH: Weinheim, Germany, 1984; pp. 139-148.

20. Kleiner, D.E.; Brunt, E.M.; van Natta, M.; Behling, C.; Contos, M.J.; Cummings, O.W.; Ferrell, L.D.; Liu, Y.-C.; Torbenson, M.S.; Unalp-Arida, A.; et al. Design and validation of a histological scoring system for nonalcoholic fatty liver disease. Hepatology 2005, 41, 1313-1321. [CrossRef] [PubMed]

21. Liang, W.; Menke, A.L.; Driessen, A.; Koek, G.H.; Lindeman, J.H.; Stoop, R.; Havekes, L.M.; Kleemann, R.; van den Hoek, A.M. Establishment of a general NAFLD scoring system for rodent models and comparison to human liver pathology. PLoS ONE 2014, 9, e115922. [CrossRef] [PubMed]

22. Manowsky, J.; Camargo, R.G.; Kipp, A.P.; Henkel, J.; Püschel, G.P. Insulin-induced cytokine production in macrophages causes insulin resistance in hepatocytes. Am. J. Physiol. Endocrin. Met. 2016, 310, E938-E946. [CrossRef] [PubMed]

23. Henkel, J.; Neuschäfer-Rube, F.; Pathe-Neuschäfer-Rube, A.; Püschel, G.P. Aggravation by prostaglandin E2 of interleukin-6-dependent insulin resistance in hepatocytes. Hepatology 2009, 50, 781-790. [CrossRef] [PubMed]

24. Xu, Z.J.; Fan, J.G.; Ding, X.D.; Qiao, L.; Wang, G.L. Characterization of high-fat, diet-induced, non-alcoholic steatohepatitis with fibrosis in rats. Digest. Dis. Sci. 2010, 55, 931-940. [CrossRef] [PubMed]

25. Matsuzawa, N.; Takamura, T.; Kurita, S.; Misu, H.; Ota, T.; Ando, H.; Yokoyama, M.; Honda, M.; Zen, Y.; Nakanuma, Y.; et al. Lipid-induced oxidative stress causes steatohepatitis in mice fed an atherogenic diet. Hepatology 2007, 46, 1392-1403. [CrossRef] [PubMed]

26. Wiggers, K.D.; Richard, M.J.; Stewart, J.W.; Jacobson, N.L.; Berger, P.J. Type and amount of dietary fat affect relative concentration of cholesterol in blood and other tissues of rats. Atherosclerosis 1977, 27, 27-34. [CrossRef]

27. Sheril, A.; Jeyakumar, S.M.; Jayashree, T.; Giridharan, N.V.; Vajreswari, A. Impact of feeding polyunsaturated fatty acids on cholesterol metabolism of dyslipidemic obese rats of WNIN/GR-Ob strain. Atherosclerosis 2009, 204, 136-140. [CrossRef] [PubMed] 
28. Schumacher, T.; Benndorf, R.A. ABC transport proteins in cardiovascular disease-A brief summary. Molecules 2017, 22, 589. [CrossRef] [PubMed]

29. Pizzini, A.; Lunger, L.; Demetz, E.; Hilbe, R.; Weiss, G.; Ebenbichler, C.; Tancevski, I. The role of omega-3 fatty acids in reverse cholesterol transport: A review. Nutrients 2017, 9, 1099. [CrossRef] [PubMed]

30. Lu, S.C.; Lin, M.H.; Huang, P.C. A high cholesterol, (n-3) polyunsaturated fatty acid diet induces hypercholesterolemia more than a high cholesterol (n-6) polyunsaturated fatty acid diet in hamsters. J. Nutr. 1996, 126, 1759-1765. [PubMed]

31. Wang, S.; Matthan, N.R.; Wu, D.; Reed, D.B.; Bapat, P.; Yin, X.; Grammas, P.; Shen, C.L.; Lichtenstein, A.H. Lipid content in hepatic and gonadal adipose tissue parallel aortic cholesterol accumulation in mice fed diets with different omega-6 PUFA to EPA plus DHA ratios. Clin. Nutr. 2014, 33, 260-266. [CrossRef] [PubMed]

32. Jump, D.B.; Depner, C.M.; Tripathy, S.; Lytle, K.A. Impact of dietary fat on the development of non-alcoholic fatty liver disease in Ldlr-/ - mice. Proc. Nutr. Soc. 2016, 75, 1-9. [CrossRef] [PubMed]

33. Shang, T.; Liu, L.; Zhou, J.; Zhang, M.; Hu, Q.; Fang, M.; Wu, Y.; Yao, P.; Gong, Z. Protective effects of various ratios of DHA/EPA supplementation on high-fat diet-induced liver damage in mice. Lipids Health Dis. 2017, 16, 65. [CrossRef] [PubMed]

34. Pachikian, B.D.; Essaghir, A.; Demoulin, J.B.; Neyrinck, A.M.; Catry, E.; de Backer, F.C.; Dejeans, N.; Dewulf, E.M.; Sohet, F.M.; Portois, L.; et al. Hepatic n-3 polyunsaturated fatty acid depletion promotes steatosis and insulin resistance in mice: Genomic analysis of cellular targets. PLoS ONE 2011, 6, e23365. [CrossRef] [PubMed]

35. Schwarz, J.; Tomé, D.; Baars, A.; Hooiveld, G.J.; Müller, M. Dietary protein affects gene expression and prevents lipid accumulation in the liver in mice. PLOS ONE 2012, 7, e47303. [CrossRef] [PubMed]

36. Legry, V.; van Rooyen, D.M.; Lambert, B.; Sempoux, C.; Poekes, L.; Español-Suñer, R.; Molendi-Coste, O.; Horsmans, Y.; Farrell, G.C.; Leclercq, I.A. Endoplasmic reticulum stress does not contribute to steatohepatitis in obese and insulin-resistant high-fat-diet-fed foz/foz mice. Clin. Sci. 2014, 127, 507-518. [CrossRef] [PubMed]

37. Ribas, V.; García-Ruiz, C.; Fernández-Checa, J.C. Glutathione and mitochondria. Front. Pharmacol. 2014, 5, 151. [CrossRef] [PubMed]

38. Marí, M.; Caballero, F.; Colell, A.; Morales, A.; Caballeria, J.; Fernandez, A.; Enrich, C.; Fernandez-Checa, J.C.; García-Ruiz, C. Mitochondrial free cholesterol loading sensitizes to TNF- and Fas-mediated steatohepatitis. Cell Metab. 2006, 4, 185-198. [CrossRef] [PubMed]

39. Schrauwen, P.; Schrauwen-Hinderling, V.; Hoeks, J.; Hesselink, M.K.C. Mitochondrial dysfunction and lipotoxicity. BBA-Mol. Cell Biol. Lipids 2010, 1801, 266-271. [CrossRef] [PubMed]

40. Bellanti, F.; Mitarotonda, D.; Tamborra, R.; Blonda, M.; Iannelli, G.; Petrella, A.; Sanginario, V.; Iuliano, L.; Vendemiale, G.; Serviddio, G. Oxysterols induce mitochondrial impairment and hepatocellular toxicity in non-alcoholic fatty liver disease. Free Radic. Biol. Med. 2014, 75, S16-S17. [CrossRef] [PubMed]

41. Vejux, A.; Guyot, S.; Montange, T.; Riedinger, J.M.; Kahn, E.; Lizard, G. Phospholipidosis and down-regulation of the PI3-K/PDK-1/Akt signalling pathway are vitamin E inhibitable events associated with 7-ketocholesterol-induced apoptosis. J. Nutr. Biochem. 2009, 20, 45-61. [CrossRef] [PubMed]

42. Ikegami, T.; Hyogo, H.; Honda, A.; Miyazaki, T.; Tokushige, K.; Hashimoto, E.; Inui, K.; Matsuzaki, Y.; Tazuma, S. Increased serum liver $\mathrm{X}$ receptor ligand oxysterols in patients with non-alcoholic fatty liver disease. J. Gastroenterol. 2012, 47, 1257-1266. [CrossRef] [PubMed]

43. Serviddio, G.; Blonda, M.; Bellanti, F.; Villani, R.; Iuliano, L.; Vendemiale, G. Oxysterols and redox signaling in the pathogenesis of non-alcoholic fatty liver disease. Free Radic. Res. 2013, 47, 881-893. [CrossRef] [PubMed]

44. Ioannou, G.N.; Haigh, W.G.; Thorning, D.; Savard, C. Hepatic cholesterol crystals and crown-like structures distinguish NASH from simple steatosis. J. Lipid Res. 2013, 54, 1326-1334. [CrossRef] [PubMed]

45. Tsuchida, T.; Friedman, S.L. Mechanisms of hepatic stellate cell activation. Nat. Rev. Gastroenterol. Hepat. 2017, 14, 397-411. [CrossRef] [PubMed]

46. Ferré, N.; Martínez-Clemente, M.; López-Parra, M.; González-Périz, A.; Horrillo, R.; Planagumà, A.; Camps, J.; Joven, J.; Tres, A.; Guardiola, F.; et al. Increased susceptibility to exacerbated liver injury in hypercholesterolemic ApoE-deficient mice: Potential involvement of oxysterols. Am. J. Physiol. Gastr. Liver Physiol. 2009, 296, G553-G562. [CrossRef] [PubMed] 
47. Ioannou, G.N.; Morrow, O.B.; Connole, M.L.; Lee, S.P. Association between dietary nutrient composition and the incidence of cirrhosis or liver cancer in the United States population. Hepatology 2009, 50, 175-184. [CrossRef] [PubMed]

48. Musso, G.; Gambino, R.; de Michieli, F.; Cassader, M.; Rizzetto, M.; Durazzo, M.; Fagà, E.; Silli, B.; Pagano, G. Dietary habits and their relations to insulin resistance and postprandial lipemia in nonalcoholic steatohepatitis. Hepatology 2003, 37, 909-916. [CrossRef] [PubMed]

49. Yasutake, K.; Nakamuta, M.; Shima, Y.; Ohyama, A.; Masuda, K.; Haruta, N.; Fujino, T.; Aoyagi, Y.; Fukuizumi, K.; Yoshimoto, T.; et al. Nutritional investigation of non-obese patients with non-alcoholic fatty liver disease: The significance of dietary cholesterol. Scand. J. Gastroenterol. 2009, 44, 471-477. [CrossRef] [PubMed]

50. Lee, H.Y.; Jun, D.W.; Kim, H.J.; Oh, H.; Saeed, W.K.; Ahn, H.; Cheung, R.C.; Nguyen, M.H. Ezetimibe decreased nonalcoholic fatty liver disease activity score but not hepatic steatosis. Korean J. Int. Med. 2018. [CrossRef] [PubMed]

51. He, X.X.; Wu, X.L.; Chen, R.P.; Chen, C.; Liu, X.G.; Wu, B.J.; Huang, Z.M. Effectiveness of omega-3 polyunsaturated fatty acids in non-alcoholic fatty liver disease: A meta-analysis of randomized controlled trials. PLoS ONE 2016, 11, e0162368. [CrossRef] [PubMed]

(C) 2018 by the authors. Licensee MDPI, Basel, Switzerland. This article is an open access article distributed under the terms and conditions of the Creative Commons Attribution (CC BY) license (http:/ / creativecommons.org/licenses/by/4.0/). 
Article

\title{
Non-Alcoholic Fatty Liver Disease in Overweight Children: Role of Fructose Intake and Dietary Pattern
}

\author{
Anika Nier ${ }^{1}$, Annette Brandt ${ }^{1}$, Ina Barbara Conzelmann ${ }^{2}$, Yelda Özel ${ }^{2}$ and Ina Bergheim ${ }^{1, *}$ \\ 1 Department of Nutritional Sciences, Molecular Nutritional Science, University of Vienna, A-1090 Vienna, \\ Austria; anika.nier@univie.ac.at (A.N.); annette.brandt@univie.ac.at (A.B.) \\ 2 Department of Nutritional Medicine, (180), University of Hohenheim, D-70599 Stuttgart, Germany; \\ ina.conzelmann@gmx.de (I.B.C.); yelda@directbox.com (Y.Ö.) \\ * Correspondence: ina.bergheim@univie.ac.at; Tel.: +43-1-4277-54981
}

Received: 26 July 2018; Accepted: 17 September 2018; Published: 19 September 2018

\begin{abstract}
The role of nutrition and diet in the development of non-alcoholic fatty liver disease (NAFLD) is still not fully understood. In the present study, we determined if dietary pattern and markers of intestinal permeability differ between overweight children with and without NAFLD. In addition, in a feasibility study, we assessed the effect of a moderate dietary intervention only focusing on nutrients identified to differ between groups on markers of intestinal barrier function and health status. Anthropometric data, dietary intake, metabolic parameters, and markers of inflammation, as well as of intestinal permeability, were assessed in overweight children $(n=89$, aged 5-9) and normal-weight healthy controls $(n=36$, aged 5-9). Sixteen children suffered from early signs of NAFLD, e.g., steatosis grade 1 as determined by ultrasound. Twelve children showing early signs of NAFLD were enrolled in the intervention study ( $n=6$ intervention, $n=6$ control). Body mass index (BMI), BMI standard deviation score (BMI-SDS), and waist circumference were significantly higher in NAFLD children than in overweight children without NAFLD. Levels of bacterial endotoxin, lipopolysaccharide-binding protein (LBP), and proinflammatory markers like interleukin 6 (IL-6) and tumor necrosis factor $\alpha$ (TNF $\alpha$ ) were also significantly higher in overweight children with NAFLD compared to those without. Total energy and carbohydrate intake were higher in NAFLD children than in those without. The higher carbohydrate intake mainly resulted from a higher total fructose and glucose intake derived from a significantly higher consumption of sugar-sweetened beverages. When counseling children with NAFLD regarding fructose intake (four times, 30-60 min within 1 year; one one-on-one counseling and three group counselings), neither alanine aminotransferase (ALT) nor aspartate aminotransferase (AST) activity in serum changed; however, diastolic blood pressure $(p<0.05)$ and bacterial endotoxin levels $(p=0.06)$ decreased markedly in the intervention group after one year. Similar changes were not found in uncounseled children. Our results suggest that a sugar-rich diet might contribute to the development of early stages of NAFLD in overweight children, and that moderate dietary counseling might improve the metabolic status of overweight children with NAFLD.
\end{abstract}

Keywords: children; overweight; NAFLD; fructose; dietary pattern; dietary intervention

\section{Introduction}

Non-alcoholic fatty liver disease (NAFLD) comprises a wide spectrum of diseases ranging from simple steatosis afflicted with fat accumulation to steatohepatitis, fibrosis, and even cirrhosis or hepatocellular carcinoma [1]. Results of epidemiological studies suggest that NAFLD is, by now, the most prevalent liver disease in the world [2]. Contrary to many other liver diseases, NAFLD is not a disease only found in adults, but, with a still increasing prevalence, is estimated to also affect $8 \%$ of normal-weight and up to $34 \%$ of overweight children and adolescents [3-5]. Despite intense research 
efforts, mechanisms underlying the onset and progression of the disease are still not fully understood, and therapies other than lifestyle interventions are not yet available. However, lifestyle interventions, be it general caloric restriction diets, or low-fat or low-carbohydrate diets with or without increased physical activity, are frequently afflicted with low compliance and high drop-out, as well as relapse rates, in both children and adults [6-8].

Similar to the results in epidemiological and clinical studies [9-12], results of animal studies analyzing the role of diet in the development of NAFLD, as well as mechanisms involved, suggest that both general overnutrition and a diet rich in certain macronutrients like saturated fat and/or sugars like fructose are critical in the onset of NAFLD [13-16]. For example, it was shown that not only obese (ob/ob) mice, but also mice fed a diet rich in saturated fats and/or fructose develop liver steatosis and early signs of hepatic inflammation within several weeks [17-19]. Furthermore, it was repeatedly shown in these dietary models that the development of NAFLD was associated with alterations of intestinal barrier function and elevated bacterial endotoxin levels. In line with these findings, results of our own group and others suggest that, in adults and children with NAFLD, the development of the disease is not only associated with general overnutriton, but also, frequently, an elevated intake of fructose and fat, as well as alterations of markers of intestinal barrier function [10,12,20-22]. However, whether or not general overnutriton or the intake of a specific dietary pattern is critical in the development of NAFLD and associated intestinal barrier dysfunction is yet to be fully clarified.

Starting from this background, the aim of the present study was to determine if the dietary pattern and lifestyle of overweight children without NAFLD differs from overweight children showing early signs of NAFLD. Accordingly, overweight children randomly recruited in primary schools, sports clubs, and kindergartens were stratified by results of liver ultrasounds in overweight children with and without NAFLD. For comparison, a group of normal-weight healthy children were enrolled in the study. Furthermore, to determine if moderate dietary counseling, focusing only on parameters identified to be critical in the dietary pattern of overweight children with NAFLD, is an approach to improve the health status of overweight children with NAFLD, some of the overweight children with NAFLD were enrolled in a feasibility study.

\section{Materials and Methods}

\subsection{Subjects}

All children were recruited from the so-called "Hohenheim Fructose Intervention (HoFI) study" registered at http:/ / clinicaltrials.gov (NCT01306396). The ethics committee of the Landesärztekammer Baden-Württemberg (Stuttgart, Germany) approved the present study, which was then performed in accordance with the ethical standards laid down in the Declaration of Helsinki (2008). Participants were recruited between April 2009 and December 2010 primarily through elementary schools in Stuttgart and the greater area of Stuttgart, Southern Germany. All subjects and their guardians gave written informed consent to participate in the study. A total of 92 overweight children aged 5-9 years without any known signs of NAFLD or other metabolic diseases before being enrolled in the study were included, while 3 children had to be excluded from the baseline analysis due to underreporting (overweight children without NAFLD: $n=2$; overweight children with NAFLD: $n=1$ ). Of the 17 overweight children identified to suffer from early stages of NAFLD, all agreed to participate in the feasibility study detailed below. Eight of the overweight children with NAFLD were selected to be in the intervention group and nine agreed to serve as controls. Despite selecting the groups, four of the 17 children enrolled in the intervention study dropped out of the study for personal reasons (three controls and one intervention child), and one child had to be excluded from the final analysis of the feasibility study as it was identified as a case of underreporting (intervention group). In addition, data from 36 healthy normal-weight children aged 5-9 reported in previously published studies [21,23] were included in the study for comparison. None of the children included had a known history of (i) steatohepatitis, (ii) renal insufficiency, (iii) diabetes type 1 and 2, (iv) chronic diseases of the 
gastrointestinal tract, or (v) taking lipid-lowering drugs or drugs affecting lipid metabolism. All children enrolled in the study were prepubertal as defined by Marshall and Tanner [24,25]. Pubertal status was assessed as described by Maier et al. [26]. None of the children enrolled in the feasibility study changed pubertal status throughout the study.

\subsection{Laboratory Measurements, Blood Pressure, and Abdominal Ultrasound}

From all participants, the following parameters were assessed in a routine laboratory (Sindelfingen, Germany) as described previously [27]: alanine aminotransferase (ALT), aspartate aminotransferase (AST), fasting blood glucose, blood lipids, and uric acid in serum, as previously described in a fasting venous blood sample. In addition, concentrations of active plasminogen activator inhibitor 1 (PAI-1; LOXO, Dossenheim, Germany), lipopolysaccharide-binding protein (LBP; Abnova, Taipei City, Taiwan), leptin and insulin (both Hölzel GmbH, Wildberg, Germany), tumor necrosis factor $\alpha$ (TNF $\alpha$; IBL international GmbH, Hamburg, Germany), adiponectin (TECOmedical AG, Sissach, Switzerland), c-reactive protein (CRP; DRG Instruments GmbH, Marburg, Germany), and interleukin 6 (IL-6; R\&D Systems, Abingdon, UK) were determined in plasma using commercially available ELISA kits. Systolic and diastolic blood pressure, as well as liver status using ultrasound, were measured and defined as detailed previously [27].

\subsection{Bacterial Endotoxin}

Plasma endotoxin levels were determined by an endpoint enzymatic assay based on limulus amebocyte lysate, as detailed previously [12].

\subsection{Glucose Metabolism}

Glucose tolerance was determined as detailed previously [26]. The homeostasis model assessment for insulin resistance $(\mathrm{HOMA}-\mathrm{IR})$ index $(\mathrm{HOMA}-\mathrm{IR}=($ fasting insulin $(\mu \mathrm{IU} / \mathrm{mL}) \times$ fasting glucose $(\mathrm{mmol} / \mathrm{L})) / 22.5)$ was used to determine insulin resistance.

\subsection{Assessment of Dietary Intake and Leisure Time Activities}

Dietary intake using two separated 24-h recalls, including one weekend day, as well as sportive and sitting leisure time activities, was assessed as previously described [28]. For analyzing nutritional intake, the EBISpro software was used. This software contains the German Nutrient Database (in German: Bundeslebensmittelschlüssel, see also https: / / www.blsdb.de) which includes data of about 10,000 foods and covers average nutritional values, including free fructose (137 constituent data per food item). Dietary underreporting was determined by calculating the ratio of reported total energy intake and predicted individual basal metabolic rate, as described previously by others [29]. Only recalls with ratios above the age- and sex-specific cut-off values being 1.04 for boys and 1.01 for girls were included in the nutritional analysis [30]. Based on this calculation, three underreporters were identified (overweight children without NAFLD: $n=2$, overweight children with NAFLD: $n=1$ ) and excluded from the final analysis and the analysis of the feasibility study, respectively.

\subsection{Anthropometric Measurements and Socio-Demographic Data}

Anthropometric parameters were assessed by a nutritionist as detailed by Maier et al. [26]. Furthermore, socio-demographic characteristics such as age, gender, and ethnicity were recorded.

\subsection{Dietary Intervention Study}

In children enrolled in the feasibility study, all measurements assessing health status and anthropometry were repeated after one year. As the feasibility study required a high compliance of participants in the intervention group, e.g., attending regular meetings (one one-on-one meeting and three group meetings), and altering dietary patterns and intake throughout the year of study, study 
participants and their guardians were allowed to choose study arms to avoid further drop-out (for study design, see also Figure 1). Based on daily fructose intake as determined in two independently performed 24-h recalls before the study, children enrolled in the intervention group were advised to reduce their daily fructose intake by $\sim 50 \%$ in a personal nutritional counseling taking place at the beginning of the study in the presence of their respective guardians. Counseling was then repeated every three months in small groups (see Figure 1). Children, as well as guardians, were counseled independently. Training of both children and guardians focused primarily on a change in dietary pattern and included strategies to identify fructose-rich foods, e.g., foods containing marked amounts of sucrose or fructose, such as lemonades, chocolates, cookies, cakes, and candies, as well as foods containing large amounts of free fructose, such as juices, certain fruits, and vegetables, and to replace these foods with foods containing less fructose of the same food category (e.g., exchanging cookies with rusk, replacing sucrose with glucose when making cakes or cookies, or to consume diet lemonades instead of sugar lemonades (for details, see Reference [27])). In brief, before the study, several games, quizzes, and experiments suitable for training children were developed and their feasibility was assessed in a pilot study [27]. For example, to illustrate the effect of sugar-sweetened beverages on dental health, a tooth was put into a glass containing a sugar-sweetened beverage for two days. Furthermore, children were asked to guess the number of sugar cubes contained in different foods (e.g., ketchup, chocolate bars, and sugar-sweetened beverages) by selecting glasses filled with different numbers of sugar cubes to enhance the awareness of sugar content in commercially available products. Additionally, a small supermarket with food dummies was built to practice children's behavior in the supermarket. Children were then asked to pick their favorite foods, and, together with a trained nutritionist, foods with high amounts of sugars were identified and alternative foods with lower sugar content were selected. Upon request, participants and their respective guardians enrolled in the control group received one dietary counseling based on general recommendations for a healthy nutrition as recommended by the German Nutrition Society.
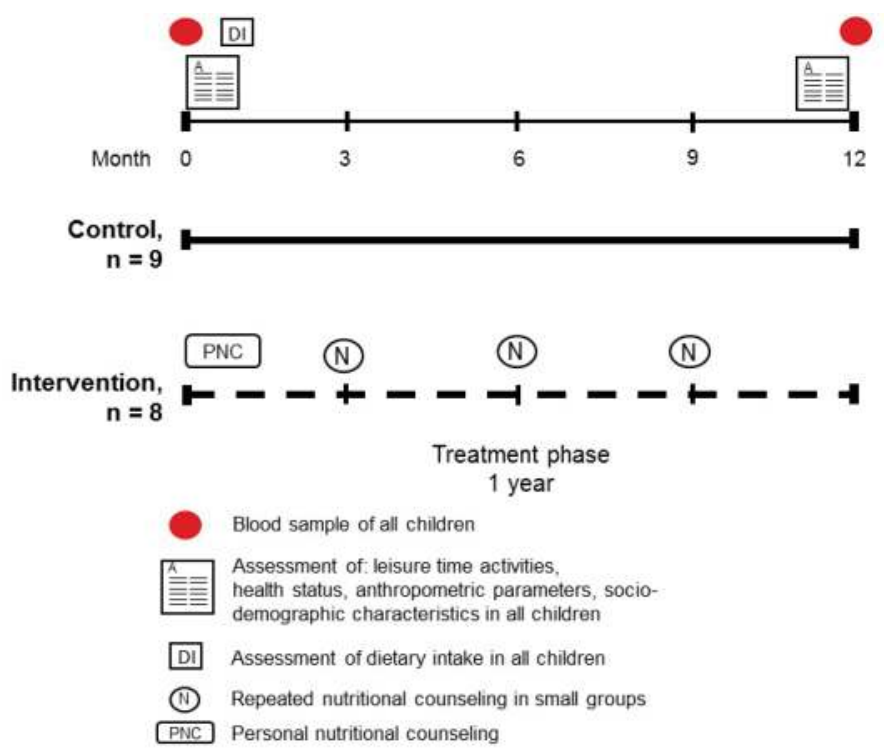

Figure 1. Study design of the feasibility study assessing the effect of a moderate dietary intervention focusing only on the reduction of fructose intake $(-50 \%)$ on the health status of overweight children with non-alcoholic fatty liver disease (NAFLD). 


\subsection{Statistical Analyses}

Data were analyzed using the $t$-test and Mann-Whitney U test for comparing overweight children with and without NAFLD and to compare baseline and end values of children enrolled in the pilot feasibility study. Healthy, normal-weight children were not included in the statistical analysis, but are shown for comparison. The Wilcoxon test was used to compare baseline and end values within the two groups enrolled in the feasibility study. Fisher's exact test was used for comparing gender and ethnicity (GraphPad Prism, version 7.03, 2017, GraphPad Software Inc., San Diego, CA, USA). Identified outliers using Grubbs' test were excluded from baseline analyses. As mentioned above, children identified as underreporters were removed from the final analyses and the analysis of the feasibility study. A $p$-value $\leq 0.05$ was defined as the level of significance.

\section{Results}

\subsection{Characteristics of the Study Participants}

As shown in Table 1, neither age nor distribution of gender or ethnicity differed between overweight children with and without early signs of NAFLD. Body weight was significantly higher in overweight children with NAFLD than in those without, while height was similar. Accordingly, body mass index (BMI), BMI standard deviation score (BMI-SDS), and waist circumference were also significantly higher in overweight children with NAFLD (see Table 1 and Figure 2). Neither leptin nor adiponectin, nor ratio of leptin and adiponectin differed between overweight groups. Still, as expected, all of these parameters differed markedly from those of normal-weight children who were included in the study for comparison only (see Table 1).

Table 1. Characteristics of normal-weight healthy children and overweight children with and without non-alcoholic fatty liver disease (NAFLD).

\begin{tabular}{cccc}
\hline & NW & OW & NAFLD \\
\hline$n$ & 36 & 73 & 16 \\
Sex (male/female) & $20 / 16$ & $33 / 40$ & $6 / 10$ \\
Ethnicity (Caucasian/Asian) & $27 / 9$ & $54 / 19$ & $8 / 8$ \\
Age (years) & $7.3 \pm 0.2$ & $7.6 \pm 0.1$ & $7.8 \pm 0.3$ \\
Weight (kg) & $27 \pm 1$ & $36 \pm 1$ & $40 \pm 2^{*}$ \\
Height (cm) & $1.26 \pm 0.01$ & $1.29 \pm 0.01$ & $1.33 \pm 0.02$ \\
BMI (kg/m ${ }^{2}$ ) & $16.7 \pm 0.2$ & $21.2 \pm 0.2$ & $22.4 \pm 0.5^{*}$ \\
BMI-SD score & $0.43 \pm 0.09$ & $1.90 \pm 0.05$ & $2.13 \pm 0.11^{*}$ \\
Waist circumference (cm) & $59 \pm 1$ & $72 \pm 1$ & $77 \pm 2^{*}$ \\
Leptin (ng/mL) & $2.3 \pm 0.3$ & $12.2 \pm 1.0$ & $9.2 \pm 1.4$ \\
Adiponectin $(\mu \mathrm{m} / \mathrm{mL})$ & $11.4 \pm 0.9$ & $10.4 \pm 0.5$ & $11.9 \pm 2.2$ \\
Leptin/Adiponectin & $0.3 \pm 0.03$ & $1.46 \pm 0.17$ & $1.21 \pm 0.26$ \\
ALT (U/L) & $19 \pm 1$ & $23 \pm 1$ & $24 \pm 2$ \\
AST (U/L) & $33 \pm 1$ & $31 \pm 1$ & $35 \pm 2^{*}$ \\
Systolic blood pressure (mmHg) & $103 \pm 1$ & $108 \pm 1$ & $111 \pm 3$ \\
Diastolic blood pressure (mmHg) & $62 \pm 1$ & $67 \pm 1$ & $70 \pm 2$ \\
Triglycerides (mg/dL) & $57 \pm 3$ & $81 \pm 4$ & $75 \pm 6$ \\
HDL cholesterol (mg/dL) & $57 \pm 1$ & $52 \pm 1$ & $48 \pm 3$ \\
LDL cholesterol (mg/dL) & $100 \pm 3$ & $117 \pm 2$ & $105 \pm 6^{*}$ \\
Total cholesterol (mg/dL) & $170 \pm 4$ & $185 \pm 3$ & $169 \pm 6^{*}$ \\
Uric Acid (mg/dL) & $3.6 \pm 0.1$ & $4.3 \pm 0.1$ & $4.1 \pm 0.2$ \\
Insulin ( $\mu$ IU/mL) & $9 \pm 0.4$ & $12 \pm 0.6$ & $16 \pm 2.3$ \\
Fasting glucose (mg/dL) & $85 \pm 1$ & $87 \pm 1$ & $85 \pm 2$ \\
HOMA-IR & $1.9 \pm 0.1$ & $2.5 \pm 0.1$ & $2.9 \pm 0.4$ \\
Physical activity (h/week) & $15 \pm 1$ & $14 \pm 1$ & $15 \pm 2$ \\
Sedentary activity (h/week) & $16 \pm 2$ & $22 \pm 1$ & $25 \pm 4$ \\
\hline
\end{tabular}

Data are shown as absolute numbers or means $\pm \mathrm{SEM},{ }^{*} p<0.05$ compared to overweight children, NW children were not included in the statistical analysis but are shown for comparison. BMI: body mass index; BMI-SD score: BMI standard deviation score; ALT: alanine aminotransferase, AST: aspartate aminotransferase; HDL: high-density lipoprotein; LDL: low-density lipoprotein; HOMA-IR: homeostatic model assessment for insulin resistance; NW: normal-weight healthy children; OW: overweight children without NAFLD; NAFLD: overweight children with NAFLD. Underreporters were excluded from the analysis. 
a

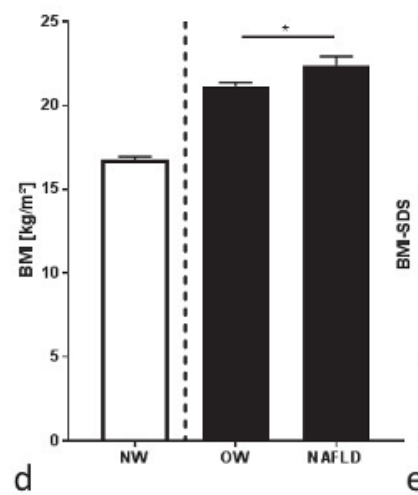

b

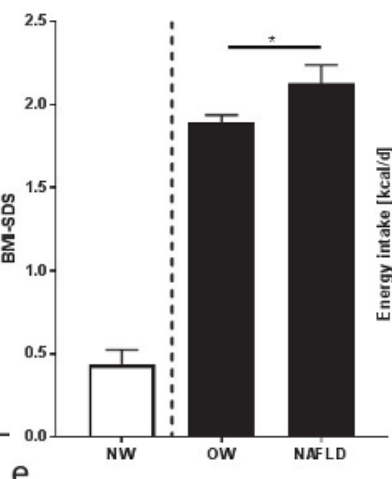

C
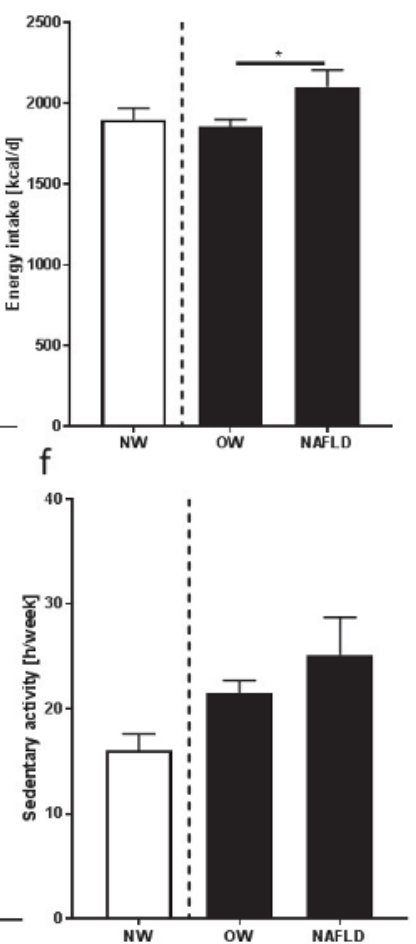

Figure 2. (a) Body mass index (BMI), (b) BMI standard deviation score (BMI-SDS), (c) energy, (d) total fructose intake (free fructose and fructose derived from sucrose), and (e) physical and (f) sedentary activities of normal-weight (NW) children, overweight children without NAFLD (OW), and overweight children with NAFLD (NAFLD). Data are means \pm standard error of the mean (SEM), ${ }^{*} p<0.05$ overweight children in comparison to overweight children with NAFLD; NW children were not included in the statistical analysis, but are shown for comparison. Underreporters were excluded from the analysis.

Despite showing early signs of NAFLD, e.g., fatty liver grade 1, ALT activity in serum was similar between overweight children with and without NAFLD, while AST activity in serum was significantly higher by $\sim 4 \mathrm{U} / \mathrm{L}(p<0.05)$. Systolic and diastolic blood pressures were similar between groups of overweight children. Twelve of the overweight children without NAFLD and seven of the overweight children with NAFLD were diagnosed as suffering from hypertension. Triglyceride, high-density lipoprotein (HDL), and uric acid concentrations in serum were also similar between the two overweight groups with 21 overweight children and four overweight children with NAFLD suffering from dyslipidemia. However, low-density lipoprotein (LDL) and total cholesterol were significantly higher in overweight children without NAFLD than in overweight children with NAFLD. Fasting insulin, glucose, and HOMA-IR were also similar between overweight groups. Furthermore, three overweight children and one overweight child with NAFLD suffered from an impaired glucose tolerance according to the reference levels of the German diabetes association [31]. As expected and similar to anthropometric parameters, metabolic parameters were markedly higher, or, in the case of HDL, lower in overweight children with and without NAFLD than in normal-weight children shown for comparison (see Table 1). 


\subsection{Nutritional Intake, Dietary Pattern, and Leisure Time Activities}

In line with the findings for body weight, BMI, and BMI-SDS, total caloric intake of overweight children with NAFLD was significantly higher than that of overweight children without NAFLD ( $250 \mathrm{kcal} /$ day). Total fat, protein, and fiber intakes were similar between overweight groups (see Table 2 and Figure 2). Total intake of carbohydrates was by trend higher in overweight children with NAFLD than in those without $(\sim 120 \mathrm{kcal} / \mathrm{day}, p=0.06)$. As results of others and our own group suggest that intake of carbohydrates and, herein, especially mono- and disaccharides may be critical in the development of NAFLD [10,32,33], the composition of the carbohydrates was further analyzed. While the intake of complex carbohydrates was similar between overweight groups, intakes of total fructose and total glucose (free fructose and glucose, respectively, as well as fructose and glucose derived from sucrose) were significantly higher in overweight children with NAFLD than in those without (see Table 2 and Figure 2).

Table 2. Nutritional intake of normal-weight healthy children and overweight children with and without NAFLD.

\begin{tabular}{cccc}
\hline & NW & OW & NAFLD \\
\hline$n$ & 36 & 73 & 16 \\
Total energy intake (kcal/day) & $1900 \pm 70$ & $1853 \pm 47$ & $2101 \pm 105^{*}$ \\
Total fat intake (g/day) & $78 \pm 4$ & $77 \pm 3$ & $86 \pm 6$ \\
Total protein intake (g/day) & $59 \pm 3$ & $63 \pm 2$ & $68 \pm 4$ \\
Total CHO intake (g/day) & $242 \pm 10$ & $227 \pm 7$ & $257 \pm 16$ \\
Fructose (g/day) a $^{\text {Glucose (g/day) }}$ & $55 \pm 3$ & $47 \pm 2$ & $61 \pm 6^{*}$ \\
Fiber intake (g/day) & $49 \pm 2$ & $42 \pm 2$ & $53 \pm 6^{*}$ \\
\hline
\end{tabular}

Data are shown as means \pm standard error of the mean (SEM); ${ }^{*} p<0.05$ compared to overweight children; NW children were not included in the statistical analysis, but are shown for comparison. CHO: carbohydrate; NW: normal-weight healthy children; OW: overweight children without NAFLD; NAFLD: overweight children with NAFLD, ${ }^{a}$ free fructose and fructose derived from sucrose; ${ }^{b}$ free glucose and glucose derived from sucrose. Underreporters were excluded from the analysis.

To further determine dietary sources of higher carbohydrate, and especially, monosaccharide intake, we analyzed the dietary pattern of study participants (see Table 3). With the exception of cereals being consumed by $\sim 47 \%$ of overweight children without NAFLD and only $\sim 13 \%$ of overweight children with NAFLD ( $p<0.05)$, the intake of food groups was similar between overweight groups (data not shown). However, when further analyzing the amount consumed of the different food groups of those children reporting consumption of these foods, overweight children with NAFLD were found to consume significantly more sweetened beverages including soft drinks and fruit juices than overweight children without NAFLD. Intake of all other food groups was similar between groups (see Table 3).

Times spent with physical and sedentary activities were similar between overweight groups. Interestingly, the intake of total calories and macronutrients, as well as time spent with physical activities, of normal-weight children was rather similar to that of overweight children with NAFLD, while time spent with sedentary activities was markedly shorter in normal-weight children than in overweight ones (see Table 1 and Figure 2). Again, underreporters were excluded from the analysis. 
Table 3. Dietary pattern of normal-weight children and overweight children with and without NAFLD.

\begin{tabular}{cccc}
\hline & NW & OW & NAFLD \\
\hline Beverages (kcal/day) & $161 \pm 18$ & $132 \pm 10$ & $208 \pm 35^{*}$ \\
Fruits/dried fruits (kcal/day) & $86 \pm 12$ & $80 \pm 7$ & $130 \pm 30$ \\
Vegetables/legumes (kcal/day) & $27 \pm 5$ & $30 \pm 4$ & $24 \pm 5$ \\
Potatoes/pasta/rice (kcal/day) & $210 \pm 33$ & $170 \pm 14$ & $201 \pm 36$ \\
Bread (kcal/day) & $259 \pm 22$ & $257 \pm 18$ & $262 \pm 48$ \\
Spreads (kcal/day) & $141 \pm 23$ & $103 \pm 10$ & $84 \pm 18$ \\
Bakery goods (kcal/day) & $206 \pm 28$ & $217 \pm 27$ & $167 \pm 33$ \\
Cereals (kcal/day) & $127 \pm 35$ & $113 \pm 12$ & $146 \pm 5$ \\
Meat (kcal/day) & $216 \pm 24$ & $205 \pm 18$ & $249 \pm 46$ \\
Milk and dairy (kcal/day) & $161 \pm 16$ & $144 \pm 11$ & $168 \pm 37$ \\
Cheese and quark (kcal/day) & $92 \pm 15$ & $104 \pm 11$ & $77 \pm 12$ \\
Oils, margarines, and butter (kcal/day) & $94 \pm 12$ & $103 \pm 9$ & $136 \pm 15$ \\
Sweets and sugar (kcal/day) & $154 \pm 26$ & $178 \pm 15$ & $145 \pm 17$ \\
Desserts (kcal/day) & $157 \pm 38$ & $111 \pm 21$ & $173 \pm 80$ \\
Convenience food (kcal/day) & $231 \pm 28$ & $324 \pm 37$ & $317 \pm 63$ \\
\hline
\end{tabular}

Data are shown as means \pm SEM; ${ }^{*} p<0.05$ compared to overweight children without NAFLD; NW children were not included in the statistical analysis, but are shown for comparison. NW: normal-weight healthy children; OW: overweight children without NAFLD; NAFLD: overweight children with NAFLD.

\subsection{Markers of Inflammation and Intestinal Permeability}

Protein concentrations of TNF $\alpha$ and IL-6 were significantly higher in plasma of overweight children with NAFLD than in those without, while concentrations of CRP and active PAI-1 in plasma were similar between overweight groups (see Figure 3). Bacterial endotoxin levels in peripheral plasma and protein levels of LBP were both significantly higher in overweight children with NAFLD than in those without (see Figure 3).

a

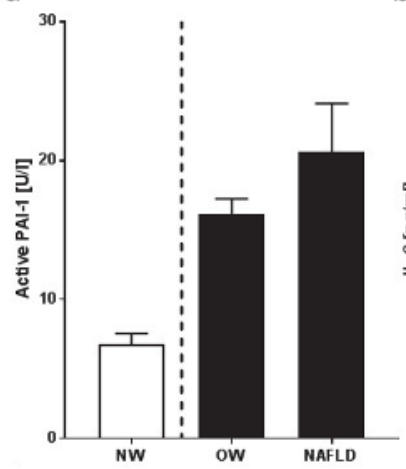

b

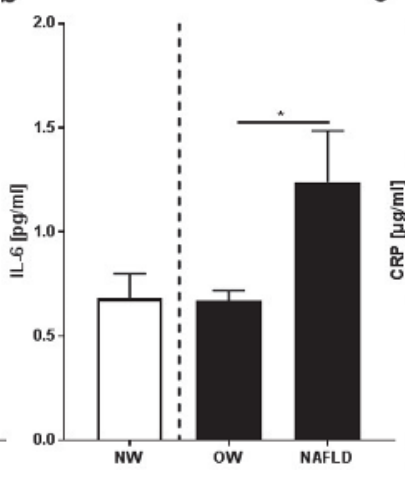

C

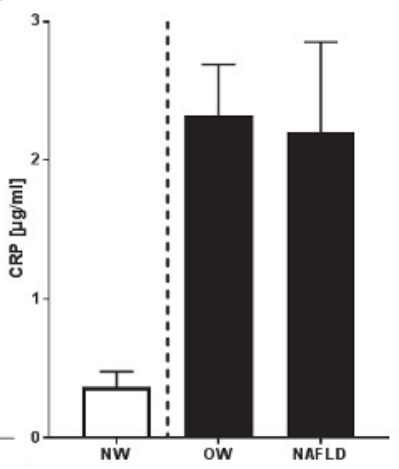

Figure 3. Cont. 

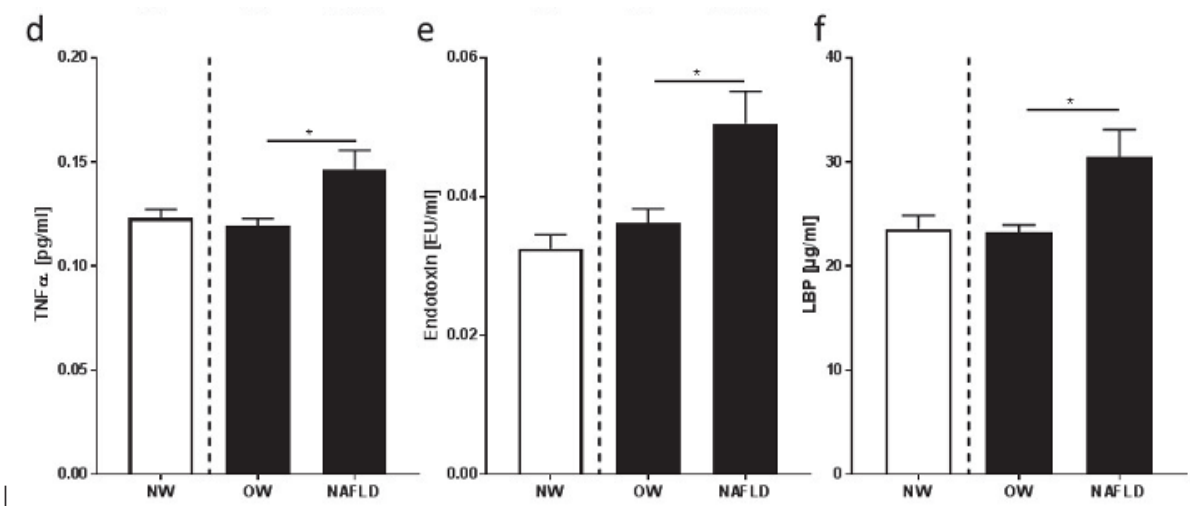

Figure 3. (a) Plasma active plasminogen activator inhibitor 1 (PAI-1), (b) interleukin 6 (IL-6), (c) serum c-reactive protein (CRP), (d) tumor necrosis factor $\alpha(\mathrm{TNF} \alpha)$, (e) endotoxin, (f) lipopolysaccharidebinding protein (LBP) plasma concentrations of normal-weight (NW) children, overweight children without NAFLD (OW), and overweight children with NAFLD (NAFLD). Data are means \pm SEM; * $p<0.05$ overweight children in comparison to overweight children with NAFLD; NW children were not included in the statistical analysis, but are shown for comparison. Underreporters were excluded from the analysis.

\subsection{Feasibility Study: Characteristics of Study Participants, Nutritional Intake, and Metabolic Parameters}

Four children dropped out of the feasibility study due to personal reasons, and one child was excluded as an underreporter. Therefore, only six children in the control group and six children in the intervention group were included in the final analysis. Despite being allowed to select study arms to enhance compliance and to avoid the loss of children, at baseline, none of the parameters assessed differed between the overweight children with NAFLD selecting the intervention arm and those who chose to participate in the feasibility study as controls, with the exception of TNF $\alpha$ levels in plasma (see Figure 4). TNF $\alpha$ levels in plasma were significantly higher in children in the intervention group than in controls. Additionally, total intakes of energy, macronutrients, and sugars (total fructose and total glucose) per day were similar between groups (see Supplementary Materials, Table S1) at baseline. Data of normal-weight children in Table 4 and Figure 4 are shown for comparison, but were not included in the statistical analysis. While both groups had lower BMI-SDS at the end of the study compared to the beginning of the study (control: $p<0.05$; intervention: $p=0.16$ ), waist circumference was significantly higher in the intervention group at the end of the study (see Table 4). A similar increase in waist circumference was not found in the controls. Nonetheless, waist circumference did not differ between groups at the end of the study. In line with the findings for waist circumference, neither adiponectin nor leptin plasma concentrations nor leptin/adiponectin ratio differed at baseline. While leptin levels remained unchanged, adiponectin concentrations in the plasma of children in the intervention group were by trend lower $(p=0.06)$ at the end of the study when compared to baseline, whereas leptin/adiponectin ratio was significantly higher in these children. 
Table 4. Anthropometric and metabolic parameters of overweight children with NAFLD before and after dietary intervention.

\begin{tabular}{|c|c|c|c|c|c|}
\hline & \multirow{3}{*}{$\begin{array}{c}\text { Healthy Children } \\
\text { NW }(n=36)\end{array}$} & \multicolumn{4}{|c|}{ NAFLD Children } \\
\hline & & \multicolumn{2}{|c|}{ Control $(n=6)$} & \multicolumn{2}{|c|}{ Intervention $(n=7)$} \\
\hline & & Baseline & After 1 Year & Baseline & After 1 Year \\
\hline Sex (male/female) & $20 / 16$ & \multicolumn{2}{|c|}{$3 / 3$} & \multicolumn{2}{|c|}{$4 / 2$} \\
\hline Ethnicity (Caucasian/Asian) & $27 / 9$ & \multicolumn{2}{|c|}{$2 / 4$} & \multicolumn{2}{|c|}{$5 / 1$} \\
\hline Age (years) & $7.3 \pm 0.2$ & $8.0 \pm 0.3$ & $9.0 \pm 0.5 *$ & $7.5 \pm 0.4$ & $8.7 \pm 0.4$ * \\
\hline BMI-SD score & $0.43 \pm 0.09$ & $1.9 \pm 0.1$ & $1.6 \pm 0.2 *$ & $2.2 \pm 0.2$ & $2.0 \pm 0.3$ \\
\hline Waist circumference $(\mathrm{cm})$ & $59 \pm 1$ & $77 \pm 4$ & $77 \pm 4$ & $76 \pm 3$ & $83 \pm 3^{*}$ \\
\hline Leptin $(\mathrm{ng} / \mathrm{mL})$ & $2.3 \pm 0.3$ & $9.5 \pm 3.0$ & $16.6 \pm 4.8$ & $8.8 \pm 2.5$ & $17.5 \pm 5.7$ \\
\hline Adiponectin $(\mu \mathrm{g} / \mathrm{mL})$ & $11.4 \pm 0.9$ & $7.6 \pm 1.4$ & $6.9 \pm 0.7$ & $18.5 \pm 4.3$ & $8.3 \pm 1.9$ \\
\hline Leptin/adiponectin & $0.3 \pm 0.03$ & $1.6 \pm 0.6$ & $2.5 \pm 0.8$ & $0.7 \pm 0.3$ & $2.0 \pm 0.3 *$ \\
\hline $\operatorname{LBP}(\mu \mathrm{g} / \mathrm{mL})$ & $23.6 \pm 1.3$ & $28.3 \pm 4.0$ & $30.2 \pm 3.2$ & $28.8 \pm 4.5$ & $25.6 \pm 2.6$ \\
\hline Active PAI-1 (U/L) & $6.8 \pm 0.8$ & $18.2 \pm 5.6$ & $18.2 \pm 8.6$ & $17.0 \pm 4.0$ & $9.6 \pm 1.4$ \\
\hline $\operatorname{ALT}(\mathrm{U} / \mathrm{L})$ & $19 \pm 1$ & $26 \pm 4$ & $26 \pm 3$ & $20 \pm 1$ & $23 \pm 3$ \\
\hline AST $(\mathrm{U} / \mathrm{L})$ & $33 \pm 1$ & $38 \pm 5$ & $30 \pm 2$ & $32 \pm 3$ & $33 \pm 3$ \\
\hline Systolic blood pressure (mmHg) & $103 \pm 1$ & $111 \pm 5$ & $113 \pm 5$ & $107 \pm 4$ & $105 \pm 5$ \\
\hline Diastolic blood pressure ( $\mathrm{mmHg}$ ) & $62 \pm 1$ & $69 \pm 3$ & $70 \pm 6$ & $69 \pm 4$ & $65 \pm 4^{*}$ \\
\hline Triglycerides $(\mathrm{mg} / \mathrm{dL})$ & $57 \pm 3$ & $80 \pm 7$ & $85 \pm 13$ & $74 \pm 15$ & $89 \pm 27$ \\
\hline HDL cholesterol (mg/dL) & $57 \pm 1$ & $48 \pm 3$ & $52 \pm 4$ & $56 \pm 4$ & $57 \pm 5$ \\
\hline LDL cholesterol (mg/dL) & $100 \pm 3$ & $109 \pm 8$ & $115 \pm 10$ & $102 \pm 13$ & $116 \pm 11 *$ \\
\hline Total cholesterol (mg/dL) & $160 \pm 4$ & $176 \pm 7$ & $174 \pm 12$ & $170 \pm 12$ & $187 \pm 10 *$ \\
\hline Insulin $(\mu \mathrm{IU} / \mathrm{mL})$ & $9 \pm 0.4$ & $13 \pm 1$ & $15 \pm 3$ & $13 \pm 4$ & $16 \pm 4$ \\
\hline Fasting glucose (mg/dL) & $85 \pm 1$ & $78 \pm 4$ & $86 \pm 3$ & $85 \pm 2$ & $86 \pm 3$ \\
\hline HOMA-IR & $1.9 \pm 0.1$ & $2.5 \pm 0.3$ & $3.3 \pm 0.8$ & $2.8 \pm 0.9$ & $3.4 \pm 0.9$ \\
\hline Physical activity (h/week) & $15 \pm 1$ & $17 \pm 4$ & $17 \pm 5$ & $14 \pm 2$ & $14 \pm 2$ \\
\hline Sedentary activity (h/week) & $16 \pm 2$ & $23 \pm 7$ & $25 \pm 4$ & $24 \pm 4$ & $18 \pm 4$ \\
\hline
\end{tabular}

Data are shown as absolute numbers or means \pm SEM; ${ }^{*} p<0.05$ compared to the respective baseline value. BMI-SD score: BMI standard deviation score; LBP: lipopolysaccharide binding protein; PAI-1: plasminogen activator inhibitor-1; ALT: alanine aminotransferase; AST: aspartate aminotransferase; HDL: high-density lipoprotein; LDL: low-density lipoprotein; HOMA-IR: homeostatic model assessment for insulin resistance. NW children were not included in the statistical analysis, but are shown for comparison. Underreporters were excluded from the analysis.

a

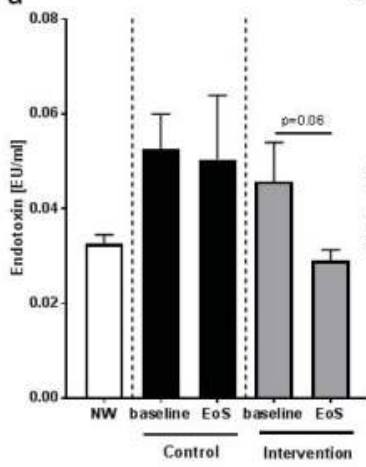

b

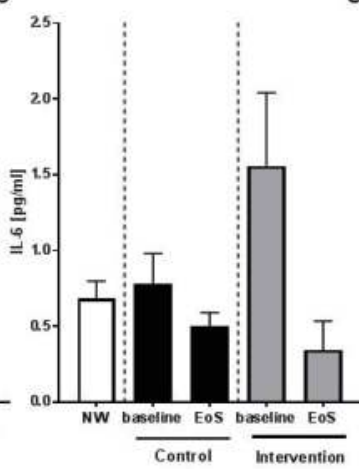

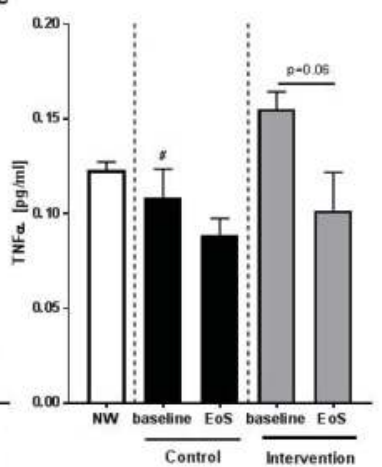

Figure 4. (a) Plasma endotoxin, (b) serum IL-6, and (c) plasma TNF $\alpha$ concentrations of children with NAFLD enrolled in the control (Control) and intervention (Intervention) group at baseline (baseline) and at the end of the study (EoS). Data are means \pm SEM; ${ }^{*} p<0.05$ values at baseline between both groups; NW children were not included in the statistical analysis, but are shown for comparison. Underreporters were excluded from the analysis.

ALT and AST activities, as well as concentrations of triglycerides and HDL in serum, were not altered throughout the intervention study and did not differ between NAFLD groups. Fasting insulin and glucose concentrations, as well as HOMA-IR, were also similar between groups at the beginning of the study and were not changed throughout the study (see Table 4). However, LDL and total cholesterol levels increased significantly throughout the study in the sera of children in the intervention group. 
LDL concentrations in serum also increased in the control group; however, as data varied considerably within these groups, differences did not reach statistical significance. The systolic blood pressure of children was similar between groups at the beginning of the study and was also not changed. In contrast, diastolic blood pressure being similar between groups at baseline was significantly lower in children in the intervention group at the end of the study, whereas a similar decrease was not found in control NAFLD children (see Table 4).

\subsection{Feasibility Study: Inflammatory Markers and Indices of Intestinal Permeability}

Concentrations of active PAI- 1 and IL-6 were similar between groups at the beginning of the study and were not altered. While changes in concentration of these inflammatory markers were not significant within groups or between groups, at the end of the feasibility study, concentrations of most parameters were at the level of normal-weight children (see Table 4 and Figure 4). In contrast, plasma TNF $\alpha$ levels, being significantly higher in the intervention group at baseline compared to controls, decreased markedly $(p=0.06)$ throughout the study almost to the level of normal-weight control children (see Figure 4). Bacterial endotoxin and LBP concentrations in plasma were also similar between groups at the beginning of the study. LBP concentration in plasma was unchanged in both groups at the end of the study (see Table 4), whereas the concentration of bacterial endotoxin in the peripheral blood of children in the intervention group was lower by trend at the end of the study $(p=0.06$; see Figure 4).

\section{Discussion}

With a still increasing prevalence, NAFLD is, by now, thought to be the most prevalent liver disease worldwide [2]. Overweight and insulin resistance are among the key risk factors for the development of NAFLD [34,35]; however, despite intense research efforts, the question as to why some overweight individuals develop NAFLD and others do not is yet to be fully answered. In the present study, employing a cohort of randomly selected overweight children with no known signs of liver disease or other metabolic diseases before the study, it was shown that children with early signs of NAFLD, e.g., fatty liver grade 1 as assessed by ultrasound, had a higher BMI, BMI-SDS, and waist circumference than overweight children without NAFLD. Also, while still being within the normal range, AST activity was higher in the sera of overweight children with NAFLD, whereas neither ALT activity nor markers of glucose metabolism nor other metabolic markers, such as blood pressure and triglycerides, differed between groups. Somewhat contrasting the findings for waist circumference, the ratio of adiponectin to leptin, suggested to be indicative of visceral fat volume and NAFLD in obese adolescents [36], was similar between overweight groups. However, results of a recently published study by Dhaliwal et al. [37] using computed tomography to assess hepatic steatosis and the abdominal fat area suggest that, while abdominal subcutaneous adipose tissue per se is greater in children with hepatic steatosis than in those without, increases in visceral adipose tissue area seem to be related to the presence of steatosis in older children ( $\geq 9.8$ years). Therefore, the apparent lack of relation of waist circumference and the ratio of adiponectin to leptin might be related to the rather young age of study participants ( $<9$ years) and to differences in subcutaneous adipose tissue mass rather than visceral adipose tissue. Furthermore, results of others also suggested that overweight children with hepatic steatosis have a higher BMI and waist circumference when compared to overweight children without signs of NAFLD [5,38,39]. Previous studies of others also reported that transaminase activities in the sera of overweight children were also similar to those of overweight children serving as controls [38]; however, in contrast to the findings of the present study, in studies of others, a strong association of the presence of NAFLD in overweight children with increased markers of insulin resistance, dyslipidemia, and the presence of metabolic abnormalities was found $[5,38,39]$. Indeed, in the present study, total cholesterol in serum was even found to be higher in overweight children without NAFLD than in those with NAFLD. Differences between the results of these studies ( $>9$ years old) and the present study ( $<9$ years old) might have resulted from 
differences in the age of study participants, as well as in the severity of steatosis (in the present study, only grade I vs. minimal-to-severe fatty liver or even beginning non-alcoholic steatohepatitis (NASH) in other studies) and obesity of participants enrolled $[5,38]$. The study setting (here, children recruited in schools vs. hospitals in most other studies) and markers used to assess insulin resistance (in the present study, fasting glucose and insulin vs. oral glucose tolerance tests) were also markedly different between these studies and the present study $[5,38,39]$. Reasons for the significantly higher levels of total cholesterol and LDL cholesterol in the sera of overweight children without NAFLD have to be delineated in future studies.

Also, while overweight children with NAFLD only showed very early signs of the disease, IL-6 and TNF $\alpha$ levels in plasma were both higher in children with NAFLD than in overweight controls, whereas CRP and active PAI-1 levels in plasma-both markedly higher than in normal-weight controls-did not differ between overweight groups. In line with these findings, others showed that concentrations of IL-6 and TNF $\alpha$ in serum are closely related to the prevalence of NALFD [40]. Results of others also suggest that, in overweight children, CRP and active PAI-1 levels may be elevated independently of the presence of NAFLD [41,42]. Indeed, while active PAI-1 levels were shown to be related to severe stages of the disease, e.g., manifest steatosis or NASH [43], this acute-phase protein was also shown to be related to HOMA-IR by others [44], and it was similarly higher in both overweight groups in the present study when compared to controls. Taken together, results of the present study suggest that, in overweight children, very early stages of NAFLD are associated with higher body weight, greater waist circumference, and elevated proinflammatory cytokine levels while, markers of insulin resistance are not different. However, the results of the present study by no means preclude that an impaired glucose tolerance or insulin resistance contributes to the onset of NAFLD. Indeed, in adults and mouse models, it was shown that both fasting insulin and glucose levels can still be within the normal range in peripheral blood, while, in liver tissue, the expressions of insulin receptor and insulin receptor substrate were markedly lower [45,46]. Therefore, it could be that, in the present study, overweight children with NAFLD may have suffered from impairments of insulin signaling and glucose metabolism in liver tissue, while fasting glucose and insulin concentrations in peripheral blood were still within the normal range. This needs to be addressed in future studies.

\subsection{Absolute Energy Intake, Nutritional Intake, and Dietary Pattern of Overweight Children with and without NAFLD Differ}

Results of animal studies suggest that not only general overnutrition, but also the composition of the diet, e.g., the proportion of saturated fatty acids and sugars, and herein, especially of fructose, may be critical in the development of NAFLD [19,47]. In a cohort of children with NAFLD, Mosca et al. [33] recently showed that dietary fructose intake is independently associated with NASH. Furthermore, it was shown that children with NAFLD absorb and metabolize fructose more effectively than normal-weight children [48]. In the present study, overweight children with early signs of NAFLD had a significantly higher mean daily total energy intake when compared to overweight children without NAFLD ( $250 \mathrm{kcal} /$ day) which mainly seemed to result from a higher daily total fructose (free fructose and fructose derived from sucrose) and total glucose (free glucose and glucose derived from sucrose) intake originating from a markedly higher soft-drink and juice intake. Results of the present study are in line with the findings of others, showing that both children and adults with NAFLD have a higher mean fructose intake mainly resulting from a higher consumption of soft drinks and fruit juices [10,49-51]; however, in most of these studies, normal-weight healthy individuals were compared with overweight patients with NAFLD [10,12]. Indeed, the number of human studies comparing the nutritional intake and dietary pattern of overweight individuals, and even more so, weight-matched individuals with and without NAFLD is rather limited. In line with the findings of the present study, Ouyang et al. and Assy et al. [52,53] showed that adult patients with NAFLD drank more soft drinks and juices. 
Interestingly, normal-weight children enrolled for comparison almost had similar daily total energy, monosaccharide, and disaccharide intakes without showing any signs of NAFLD or other metabolic diseases when compared to overweight children with NAFLD. Yet, normal weight children, on average, were $\sim 16 \mathrm{~h} /$ week ( $140 \mathrm{~min} /$ day) sedentarily active doing handcrafting, drawing, reading, and watching TV or playing video games, while overweight children with NAFLD, on average, spent $25 \mathrm{~h} /$ week ( $215 \mathrm{~min} /$ day) with these activities. Indeed, results of Felix et al. [54] suggest that, in overweight children, the intake of refined carbohydrates and the lack of physical activity were associated with a higher risk of developing NAFLD, further suggesting that protection against the development of NAFLD, and probably, overweight in normal-weight children was strongly dependent upon their physical activity in the present study. In support of this hypothesis, lifestyle changes not only focusing on changes in dietary habits, but also on increasing aerobic exercise were suggested to improve aminotransferase activity levels in children and adolescents with NAFLD [50,55].

4.2. Overweight Children with NAFLD Have Higher Bacterial Endotoxin and LBP Levels in Peripheral Blood Than Overweight Children without NAFLD, Which Were Lowered by Moderate Dietary Counseling

In the present study, both bacterial endotoxin and LBP levels were significantly higher in overweight children with NAFLD than in those without. These findings are in line with the results of several human and animal studies, suggesting that alterations of intestinal barrier function, and subsequently, an increased translocation of bacterial endotoxin are critical in the development of NAFLD $[10,12,17,21,56-58]$. While data derived from animal studies suggest that these alterations may be related to the intake of fructose $[14,19,58]$, results of human studies are somewhat contradictory. Indeed, in some studies, it was shown that both the intake of dietary fructose and bacterial endotoxin levels in peripheral blood were elevated in patients with NAFLD; however, frequently, not only fructose intake, but also total caloric intake of patients was significantly higher than that of controls $[49,53]$. So far, studies focusing on a reduction of fructose intake suggest that, in adults with steatosis and steatohepatitis, this kind of counseling is associated with an improvement in liver status, a reduction in bacterial endotoxin levels, and improved intestinal barrier function [59]. In the present feasibility study, while only slightly affecting overall weight and metabolic status, the moderate dietary counseling focusing only on a reduction in dietary fructose intake was associated with a reduction in bacterial endotoxin and TNF $\alpha$ levels, almost to the level of normal-weight controls, in overweight children with NAFLD. The apparent reduction in adiponectin levels from baseline to the end of study in children with NAFLD enrolled in the intervention arm might have resulted from the slightly, but not significantly, younger age of these children when compared to controls at baseline (7.5 vs. 8.0 years). Indeed, it was shown before that adiponectin plasma levels decrease between the ages of five and eight years [60]. Furthermore, studies of Murphy et al. [60] also showed that total cholesterol plasma levels increase over time in children aged between five and eight years, in line with findings of the present study. In contrast, despite lowering their BMI-SDS and maintaining their waist circumference, the bacterial endotoxin levels of overweight controls with NAFLD were unchanged. Taken together, these data suggest that the total intake of sugar-rich foods and bacterial endotoxin levels both may be critical in the development of NAFLD in overweight children. However, our results do not preclude that other factors such as genetic predisposition, intake of other nutrients, and sedentary lifestyle are also critical in the development of NAFLD. Rather, our data suggest that, at least in some children, targeting intestinal barrier function through dietary fructose intake may be beneficial in the prevention and therapy of this liver disease.

\subsection{Limitations}

Our study is not without limitations which have to be considered when interpreting the results. Overweight children with and without NAFLD were not weight-matched; however, children were randomly recruited and enrolled in non-clinical settings, and, at the time of recruitment, had no known history of metabolic or liver diseases. Therefore, both overweight groups included metabolically 
healthy and unhealthy children. Thus, results might differ in larger and more homogeneous clinical studies. Nonetheless, as we aimed to study the early onset of the disease, this approach seemed to be the most feasible. Furthermore, the sample size of the intervention study was rather small, as the intervention focused only on children with NAFLD and five children were lost due to personal reasons or underreporting. Furthermore, as the focus of the intervention study was to show feasibility of this kind of moderate dietary intervention, no power calculation was performed to determine the number of subjects needed to be included for statistically significant outcomes. Thus, the characteristics of the feasibility study are rather explorative, and the effect of a moderate dietary intervention on metabolic and inflammatory markers needs to be assured in a larger randomized population. However, despite the small sample size, our findings are in line with others showing that dietary counseling might be beneficial in improving metabolic parameters in children [61]. Furthermore, a selection bias cannot be ruled out as control and intervention groups were self-selected due to incompliance of many guardians for randomization. Indeed, due to drop out and underreporting, the number of Asian participants deciding to undergo nutritional counseling was lower than the number of Asian children in the control group. However, as we were highly dependent upon the willingness of parents and children to attend the regular counseling meetings, from our perspective, this was the most feasible way of avoiding an even higher drop-out rate. Indeed, when enrolling children into the study, guardians repeatedly pointed out their unwillingness to participate in regular meetings, suggesting that it felt too straining. Accordingly, it was also not possible to obtain valid data regarding nutritional intake and dietary pattern at the end of the intervention. Therefore, it is not clear if the beneficial effects on bacterial endotoxin levels found at the end of the study resulted from a change in fructose intake or dietary pattern, or other factors. The role and impact of fructose on the beneficial effects found in the intervention group will have to be addressed in future studies. Reasons for this incompliance to participate in meetings might have been that our study was not situated in a clinical setting, and that children were thought to be healthy with the exception of being overweight/ obese before the study. Another limitation is the reduction of BMI-SDS in both groups of the intervention study. This, in part, might have resulted from the fact that guardians became aware of the potential health issues of their children. Indeed, some of the families might have changed additional dietary habits, and prolonged time spent physically active, while time spent sedentary active was reduced, without bringing this to our attention. Furthermore, in the region of Germany where our study was situated, "healthy" nutrition is part of the curriculum in elementary school and sometimes even in kindergarten. Therefore, it cannot be ruled out that at least some of the children in the intervention study and probably also their parents, e.g., during parent-teacher conferences and school enrollment, might have received additional training in regards to avoiding sugar-rich foods and to following a healthy lifestyle. Furthermore, in the present study, physical and sedentary activities were only acquired by questionnaires rather than activity monitors. Additionally, no follow-up was carried out to assess sustainability of the intervention on weight status, metabolic disorders, and associated proinflammatory alterations. However, we thought that the length of the study would be sufficient to test the principal hypothesis that, in children, a diet focusing only on a reduction in fructose intake may be a sufficient measure for reducing bacterial endotoxin levels and concentrations of proinflammatory cytokines. Long-term effects will have to be determined in larger randomized studies with a longer duration and follow-up.

\section{Conclusions}

Taken together, results of the present study suggest that body weight, dietary pattern, and especially, the intake of sweetened beverages may be critical in the development of NAFLD in overweight children. Our data also suggest that changes in intestinal barrier function are also associated with the development of NAFLD in children. Results of the present study also suggest that targeting sugar or fructose intake even with moderate measures may be beneficial for overall health status of overweight children with NAFLD. Still, the concept of moderate lifestyle interventions only focusing on a limited number of changes and the long-term effects of interventions like the one used in the 
present study need to be assessed in larger randomized studies in the future, as, in the present study, the sample size was quite small and participants were allowed to self-select their groups. Also, future studies should maybe aim to include more family members in the intervention, so as to improve the motivation to participate in and attend regular meetings, as well as to translate information and advice provided during the counseling and regular meetings to daily life, subsequently leading to a better compliance and greater health effects. In addition, employing new dietary counseling tools not requiring physical presence in a study center may also be an option for future studies. Furthermore, mechanisms underlying the elevated bacterial endotoxin levels found in the present study, as well as other studies, remain to be determined.

Supplementary Materials: The following are available online at http:/ / www.mdpi.com/2072-6643/10/9/1329/ s1, Table S1: Nutritional intake of overweight children with NAFLD enrolled in the feasibility study at baseline.

Author Contributions: Conceptualization, I.B. Data curation, A.N., A.B., I.B.C., and Y.Ö. Formal analysis, A.N., A.B., I.B.C., and Y.Ö. Funding acquisition, I.B. Investigation, I.B.C. and Y.Ö. Project administration, I.B. Supervision, I.B. Validation, I.B. Visualization, A.N. Writing—original draft, A.N. and I.B. Writing—review and editing, I.B.

Funding: This research was funded by the German Ministry of Education and Science (BMBF), FKZ: 01EA1305 (IB).

Conflicts of Interest: The authors declare no conflicts of interest. The funders had no role in the design of the study; in the collection, analyses, or interpretation of data; in the writing of the manuscript, and in the decision to publish the results.

\section{References}

1. Angulo, P.; Lindor, K.D. Non-alcoholic fatty liver disease. J. Gastroenterol. Hepatol. 2002, 17, S186-S190. [CrossRef] [PubMed]

2. Younossi, Z.; Anstee, Q.M.; Marietti, M.; Hardy, T.; Henry, L.; Eslam, M.; George, J.; Bugianesi, E. Global burden of nafld and nash: Trends, predictions, risk factors and prevention. Nat. Rev. Gastroenterol. Hepatol. 2018, 15, 11-20. [CrossRef] [PubMed]

3. Alkassabany, Y.M.; Farghaly, A.G.; El-Ghitany, E.M. Prevalence, risk factors, and predictors of nonalcoholic fatty liver disease among schoolchildren: A hospital-based study in alexandria, egypt. Arab. J. Gastroenterol. 2014, 15, 76-81. [CrossRef] [PubMed]

4. Anderson, E.L.; Howe, L.D.; Jones, H.E.; Higgins, J.P.T.; Lawlor, D.A.; Fraser, A. The prevalence of non-alcoholic fatty liver disease in children and adolescents: A systematic review and meta-analysis. PLoS ONE 2015, 10, e0140908. [CrossRef] [PubMed]

5. Denzer, C.; Thiere, D.; Muche, R.; Koenig, W.; Mayer, H.; Kratzer, W.; Wabitsch, M. Gender-specific prevalences of fatty liver in obese children and adolescents: Roles of body fat distribution, sex steroids, and insulin resistance. J. Clin. Endocrinol. Metab. 2009, 94, 3872-3881. [CrossRef] [PubMed]

6. Denzer, C.; Reithofer, E.; Wabitsch, M.; Widhalm, K. The outcome of childhood obesity management depends highly upon patient compliance. Eur. J. Pediatr. 2004, 163, 99-104. [CrossRef] [PubMed]

7. McManus, K.; Antinoro, L.; Sacks, F. A randomized controlled trial of a moderate-fat, low-energy diet compared with a low fat, low-energy diet for weight loss in overweight adults. Int. J. Obes. Relat. Metab. Disord. 2001, 25, 1503-1511. [CrossRef] [PubMed]

8. Reinehr, T. Effectiveness of Lifestyle Intervention in Overweight Children. Proc. Nutr. Soc. 2011, 70, 494-505. [CrossRef] [PubMed]

9. Sullivan, S. Implications of diet on nonalcoholic fatty liver disease. Curr. Opin. Gastroenterol. 2010, 26, 160-164. [CrossRef] [PubMed]

10. Thuy, S.; Ladurner, R.; Volynets, V.; Wagner, S.; Strahl, S.; Konigsrainer, A.; Maier, K.P.; Bischoff, S.C.; Bergheim, I. Nonalcoholic fatty liver disease in humans is associated with increased plasma endotoxin and plasminogen activator inhibitor 1 concentrations and with fructose intake. J. Nutr. 2008, 138, 1452-1455. [CrossRef] [PubMed]

11. Toshimitsu, K.; Matsuura, B.; Ohkubo, I.; Niiya, T.; Furukawa, S.; Hiasa, Y.; Kawamura, M.; Ebihara, K.; Onji, M. Dietary habits and nutrient intake in non-alcoholic steatohepatitis. Nutrition 2007, 23, 46-52. [CrossRef] [PubMed] 
12. Volynets, V.; Kuper, M.A.; Strahl, S.; Maier, I.B.; Spruss, A.; Wagnerberger, S.; Konigsrainer, A.; Bischoff, S.C.; Bergheim, I. Nutrition, intestinal permeability, and blood ethanol levels are altered in patients with nonalcoholic fatty liver disease (nafld). Dig. Dis. Sci. 2012, 57, 1932-1941. [CrossRef] [PubMed]

13. Ackerman, Z.; Oron-Herman, M.; Grozovski, M.; Rosenthal, T.; Pappo, O.; Link, G.; Sela, B.A. Fructose-induced fatty liver disease: Hepatic effects of blood pressure and plasma triglyceride reduction. Hypertension 2005, 45, 1012-1018. [CrossRef] [PubMed]

14. Bergheim, I.; Weber, S.; Vos, M.; Kramer, S.; Volynets, V.; Kaserouni, S.; McClain, C.J.; Bischoff, S.C. Antibiotics protect against fructose-induced hepatic lipid accumulation in mice: Role of endotoxin. J. Hepatol. 2008, 48, 983-992. [CrossRef] [PubMed]

15. Kanuri, G.; Spruss, A.; Wagnerberger, S.; Bischoff, S.C.; Bergheim, I. Fructose-induced steatosis in mice: Role of plasminogen activator inhibitor-1, microsomal triglyceride transfer protein and nkt cells. Lab. Investig. 2011, 91, 885-895. [CrossRef] [PubMed]

16. Spruss, A.; Bergheim, I. Dietary fructose and intestinal barrier: Potential risk factor in the pathogenesis of nonalcoholic fatty liver disease. J. Nutr. Biochem. 2009, 20, 657-662. [CrossRef] [PubMed]

17. Brandt, A.; Jin, C.J.; Nolte, K.; Sellmann, C.; Engstler, A.J.; Bergheim, I. Short-term intake of a fructose-, fatand cholesterol-rich diet causes hepatic steatosis in mice: Effect of antibiotic treatment. Nutrients 2017, 9. [CrossRef] [PubMed]

18. Kanuri, G.; Bergheim, I. In vitro and in vivo models of non-alcoholic fatty liver disease (nafld). Int. J. Mol. Sci. 2013, 14, 11963-11980. [CrossRef] [PubMed]

19. Sellmann, C.; Priebs, J.; Landmann, M.; Degen, C.; Engstler, A.J.; Jin, C.J.; Garttner, S.; Spruss, A.; Huber, O.; Bergheim, I. Diets rich in fructose, fat or fructose and fat alter intestinal barrier function and lead to the development of nonalcoholic fatty liver disease over time. J. Nutr. Biochem. 2015, 26, 1183-1192. [CrossRef] [PubMed]

20. Hashemi Kani, A.; Alavian, S.M.; Esmaillzadeh, A.; Adibi, P.; Azadbakht, L. Dietary quality indices and biochemical parameters among patients with non alcoholic fatty liver disease (nafld). Hepat. Mon. 2013, 13, e10943. [CrossRef] [PubMed]

21. Nier, A.; Engstler, A.J.; Maier, I.B.; Bergheim, I. Markers of intestinal permeability are already altered in early stages of non-alcoholic fatty liver disease: Studies in children. PLoS ONE 2017, 12, e0183282. [CrossRef] [PubMed]

22. Shi, L.; Liu, Z.W.; Li, Y.; Gong, C.; Zhang, H.; Song, L.J.; Huang, C.Y.; Li, M. The prevalence of nonalcoholic fatty liver disease and its association with lifestyle/dietary habits among university faculty and staff in chengdu. Biomed. Environ. Sci. 2012, 25, 383-391. [CrossRef] [PubMed]

23. Engstler, A.J.; Aumiller, T.; Degen, C.; Durr, M.; Weiss, E.; Maier, I.B.; Schattenberg, J.M.; Jin, C.J.; Sellmann, C.; Bergheim, I. Insulin resistance alters hepatic ethanol metabolism: Studies in mice and children with non-alcoholic fatty liver disease. Gut 2016, 65, 1564-1571. [CrossRef] [PubMed]

24. Marshall, W.A.; Tanner, J.M. Variations in pattern of pubertal changes in girls. Arch. Dis. Child. 1969, 44, 291-303. [CrossRef] [PubMed]

25. Marshall, W.A.; Tanner, J.M. Variations in the pattern of pubertal changes in boys. Arch. Dis. Child. 1970, 45, 13-23. [CrossRef] [PubMed]

26. Maier, I.B.; Ozel, Y.; Engstler, A.J.; Puchinger, S.; Wagnerberger, S.; Hulpke-Wette, M.; Bischoff, S.C.; Bergheim, I. Differences in the prevalence of metabolic disorders between prepubertal boys and girls from 5 to 8 years of age. Acta Paediatr. 2014, 103, e154-e160. [CrossRef] [PubMed]

27. Maier, I.B.; Stricker, L.; Ozel, Y.; Wagnerberger, S.; Bischoff, S.C.; Bergheim, I. A low fructose diet in the treatment of pediatric obesity: A pilot study. Pediatr. Int. 2011, 53, 303-308. [CrossRef] [PubMed]

28. Maier, I.B.; Ozel, Y.; Wagnerberger, S.; Bischoff, S.C.; Bergheim, I. Dietary pattern and leisure time activity of overweight and normal weight children in germany: Sex-specific differences. Nutr. J. 2013, 12, 14. [CrossRef] [PubMed]

29. Schofield, W.N. Predicting basal metabolic rate, new standards and review of previous work. Hum. Nutr. Clin. Nutr. 1985, 39 (Suppl. 1), 5-41. [PubMed]

30. Sichert-Hellert, W.; Kersting, M.; Schoch, G. Underreporting of energy intake in 1 to 18 year old german children and adolescents. Z. Ernahrungswiss. 1998, 37, 242-251. [CrossRef] [PubMed]

31. DDG. Evidenzbasierte Leitlinie der Deutschen Diabetes-Gesellschaft. Available online: www.deutschediabetes-gesellschaft.de (accessed on 20 July 2018). 
32. Mager, D.R.; Patterson, C.; So, S.; Rogenstein, C.D.; Wykes, L.J.; Roberts, E.A. Dietary and physical activity patterns in children with fatty liver. Eur. J. Clin. Nutr. 2010, 64, 628-635. [CrossRef] [PubMed]

33. Mosca, A.; Nobili, V.; De Vito, R.; Crudele, A.; Scorletti, E.; Villani, A.; Alisi, A.; Byrne, C.D. Serum uric acid concentrations and fructose consumption are independently associated with nash in children and adolescents. J. Hepatol. 2017, 66, 1031-1036. [CrossRef] [PubMed]

34. D'Adamo, E.; Impicciatore, M.; Capanna, R.; Loredana Marcovecchio, M.; Masuccio, F.G.; Chiarelli, F.; Mohn, A.A. Liver steatosis in obese prepubertal children: A possible role of insulin resistance. Obesity 2008, 16, 677-683. [CrossRef] [PubMed]

35. Nath, P.; Singh, S.P. Nonalcoholic fatty liver disease: Time to take the bull by the horns. Eur. J. Hepatol.-Gastroenterol. 2018, 8, 47-51. [CrossRef] [PubMed]

36. Angin, Y.; Arslan, N.; Kuralay, F. Leptin-to-adiponectin ratio in obese adolescents with nonalcoholic fatty liver disease. Turk. J. Pediatr. 2014, 56, 259-266. [PubMed]

37. Dhaliwal, J.; Chavhan, G.B.; Lurz, E.; Shalabi, A.; Yuen, N.; Williams, B.; Martincevic, I.; Amirabadi, A.; Wales, P.W.; Lee, W.; et al. Hepatic steatosis is highly prevalent across the paediatric age spectrum, including in pre-school age children. Aliment. Pharmacol. Ther. 2018. [CrossRef] [PubMed]

38. Papandreou, D.; Karavetian, M.; Karabouta, Z.; Andreou, E. Obese children with metabolic syndrome have 3 times higher risk to have nonalcoholic fatty liver disease compared with those without metabolic syndrome. Int. J. Endocrinol. 2017, 2017, 2671692. [CrossRef] [PubMed]

39. Prokopowicz, Z.; Malecka-Tendera, E.; Matusik, P. Predictive value of adiposity level, metabolic syndrome, and insulin resistance for the risk of nonalcoholic fatty liver disease diagnosis in obese children. Can. J. Gastroenterol. Hepatol. 2018, 2018, 9465784. [CrossRef] [PubMed]

40. Alisi, A.; Manco, M.; Devito, R.; Piemonte, F.; Nobili, V. Endotoxin and plasminogen activator inhibitor-1 serum levels associated with nonalcoholic steatohepatitis in children. J. Pediatr. Gastroenterol. Nutr. 2010, 50, 645-649. [CrossRef] [PubMed]

41. Assuncao, S.N.F.; Sorte, N.; Alves, C.A.D.; Mendes, P.S.A.; Alves, C.R.B.; Silva, L.R. Inflammatory cytokines and non-alcoholic fatty liver disease (nafld) in obese children and adolescents. Nutr. Hosp. 2018, 35, 78-83. [CrossRef] [PubMed]

42. Estelles, A.; Dalmau, J.; Falco, C.; Berbel, O.; Castello, R.; Espana, F.; Aznar, J. Plasma pai-1 levels in obese children-Effect of weight loss and influence of pai-1 promoter $4 \mathrm{~g} / 5 \mathrm{~g}$ genotype. Thromb. Haemost. 2001, 86, 647-652. [PubMed]

43. Fitzpatrick, E.; Dew, T.K.; Quaglia, A.; Sherwood, R.A.; Mitry, R.R.; Dhawan, A. Analysis of adipokine concentrations in paediatric non-alcoholic fatty liver disease. Pediatr. Obes. 2012, 7, 471-479. [CrossRef] [PubMed]

44. Masquio, D.C.; de Piano, A.; Campos, R.M.; Sanches, P.L.; Corgosinho, F.C.; Carnier, J.; Oyama, L.M.; do Nascimento, C.M.; de Mello, M.T.; Tufik, S.; et al. Saturated fatty acid intake can influence increase in plasminogen activator inhibitor-1 in obese adolescents. Horm. Metab. Res. 2014, 46, 245-251. [CrossRef] [PubMed]

45. Jin, C.J.; Sellmann, C.; Engstler, A.J.; Ziegenhardt, D.; Bergheim, I. Supplementation of sodium butyrate protects mice from the development of non-alcoholic steatohepatitis (nash). Br. J. Nutr. 2015, 114, 1745-1755. [CrossRef] [PubMed]

46. Kanuri, G.; Ladurner, R.; Skibovskaya, J.; Spruss, A.; Konigsrainer, A.; Bischoff, S.C.; Bergheim, I. Expression of toll-like receptors 1-5 but not th 6-10 is elevated in livers of patients with non-alcoholic fatty liver disease. Liver. Int. 2015, 35, 562-568. [CrossRef] [PubMed]

47. Kim, T.H.; Choi, D.; Kim, J.Y.; Lee, J.H.; Koo, S.H. Fast food diet-induced non-alcoholic fatty liver disease exerts early protective effect against acetaminophen intoxication in mice. BMC Gastroenterol. 2017, 17, 124. [CrossRef] [PubMed]

48. Sullivan, J.S.; Le, M.T.; Pan, Z.; Rivard, C.; Love-Osborne, K.; Robbins, K.; Johnson, R.J.; Sokol, R.J.; Sundaram, S.S. Oral fructose absorption in obese children with non-alcoholic fatty liver disease. Pediatr. Obes. 2015, 10, 188-195. [CrossRef] [PubMed]

49. Abdelmalek, M.F.; Suzuki, A.; Guy, C.; Unalp-Arida, A.; Colvin, R.; Johnson, R.J.; Diehl, A.M.; Clini, N.S. Increased fructose consumption is associated with fibrosis severity in patients with nonalcoholic fatty liver disease. Hepatology 2010, 51, 1961-1971. [CrossRef] [PubMed] 
50. Utz-Melere, M.; Targa-Ferreira, C.; Lessa-Horta, B.; Epifanio, M.; Mouzaki, M.; Mattos, A.A. Non-alcoholic fatty liver disease in children and adolescents: Lifestyle change-A systematic review and meta-analysis. Ann. Hepatol. 2018, 17, 345-354. [CrossRef] [PubMed]

51. Zelber-Sagi, S.; Nitzan-Kaluski, D.; Goldsmith, R.; Webb, M.; Blendis, L.; Halpern, Z.; Oren, R. Long term nutritional intake and the risk for non-alcoholic fatty liver disease (nafld): A population based study. J. Hepatol. 2007, 47, 711-717. [CrossRef] [PubMed]

52. Assy, N.; Nasser, G.; Kamayse, I.; Nseir, W.; Beniashvili, Z.; Djibre, A.; Grosovski, M. Soft drink consumption linked with fatty liver in the absence of traditional risk factors. Can. J. Gastroenterol. 2008, 22, 811-816. [CrossRef] [PubMed]

53. Ouyang, X.; Cirillo, P.; Sautin, Y.; McCall, S.; Bruchette, J.L.; Diehl, A.M.; Johnson, R.J.; Abdelmalek, M.F. Fructose consumption as a risk factor for non-alcoholic fatty liver disease. J. Hepatol. 2008, 48, 993-999. [CrossRef] [PubMed]

54. Félix, D.R.; Costenaro, F.; Gottschall, C.B.A.; Coral, G.P. Non-alcoholic fatty liver disease (nafld) in obese children- effect of refined carbohydrates in diet. BMC Pediatr. 2016, 16, 187. [CrossRef] [PubMed]

55. Medrano, M.; Cadenas-Sanchez, C.; Alvarez-Bueno, C.; Cavero-Redondo, I.; Ruiz, J.R.; Ortega, F.B.; Labayen, I. Evidence-based exercise recommendations to reduce hepatic fat content in youth- a systematic review and meta-analysis. Prog. Cardiovasc. Dis. 2018. [CrossRef] [PubMed]

56. Guercio Nuzio, S.; Di Stasi, M.; Pierri, L.; Troisi, J.; Poeta, M.; Bisogno, A.; Belmonte, F.; Tripodi, M.; Di Salvio, D.; Massa, G.; et al. Multiple gut-liver axis abnormalities in children with obesity with and without hepatic involvement. Pediatr. Obes. 2016. [CrossRef]

57. Harte, A.L.; da Silva, N.F.; Creely, S.J.; McGee, K.C.; Billyard, T.; Youssef-Elabd, E.M.; Tripathi, G.; Ashour, E.; Abdalla, M.S.; Sharada, H.M.; et al. Elevated endotoxin levels in non-alcoholic fatty liver disease. J. Inflamm. 2010, 7, 15. [CrossRef] [PubMed]

58. Ouelaa, W.; Jegatheesan, P.; M’Bouyou-Boungou, J.; Vicente, C.; Nakib, S.; Nubret, E.; De Bandt, J.P. Citrulline decreases hepatic endotoxin-induced injury in fructose-induced non-alcoholic liver disease: An ex vivo study in the isolated perfused rat liver. Br. J. Nutr. 2017, 117, 1487-1494. [CrossRef] [PubMed]

59. Volynets, V.; Machann, J.; Kuper, M.A.; Maier, I.B.; Spruss, A.; Konigsrainer, A.; Bischoff, S.C.; Bergheim, I. A moderate weight reduction through dietary intervention decreases hepatic fat content in patients with non-alcoholic fatty liver disease (nafld): A pilot study. Eur. J. Nutr. 2013, 52, 527-535. [CrossRef] [PubMed]

60. Murphy, M.J.; Hosking, J.; Metcalf, B.S.; Voss, L.D.; Jeffery, A.N.; Sattar, N.; Williams, R.; Jeffery, J.; Wilkin, T.J. Distribution of adiponectin, leptin, and metabolic correlates of insulin resistance: A longitudinal study in british children; 1: Prepuberty (earlybird 15). Clin. Chem. 2008, 54, 1298-1306. [CrossRef] [PubMed]

61. Chen, A.K.; Roberts, C.K.; Barnard, R.J. Effect of a short-term diet and exercise intervention on metabolic syndrome in overweight children. Metabolism 2006, 55, 871-878. [CrossRef] [PubMed]

(C) 2018 by the authors. Licensee MDPI, Basel, Switzerland. This article is an open access article distributed under the terms and conditions of the Creative Commons Attribution (CC BY) license (http:/ / creativecommons.org/licenses/by/4.0/). 


\title{
Communication \\ Gut Permeability Might be Improved by Dietary Fiber in Individuals with Nonalcoholic Fatty Liver Disease (NAFLD) Undergoing Weight Reduction
}

\author{
Marcin Krawczyk ${ }^{1,2}{ }^{(D}$, Dominika Maciejewska ${ }^{3}$, Karina Ryterska ${ }^{3}{ }^{\oplus}$, \\ Maja Czerwińka-Rogowska ${ }^{3} \mathbb{D}$, Dominika Jamioł-Milc ${ }^{3}$, Karolina Skonieczna-Żydecka ${ }^{3}$ (D), \\ Piotr Milkiewicz ${ }^{4,5}$, Joanna Raszeja-Wyszomirska ${ }^{4}$ and Ewa Stachowska ${ }^{3, *(D)}$ \\ 1 Department of Medicine II, Saarland University Medical Center, Saarland University, \\ 66421 Homburg, Germany; marcin.krawczyk@uks.eu \\ 2 Laboratory of Metabolic Liver Diseases, Centre for Preclinical Research, Department of General, Transplant \\ and Liver Surgery, Medical University of Warsaw, 02-097 Warsaw, Poland \\ 3 Department of Biochemistry and Human Nutrition, Pomeranian Medical University, \\ 71-210 Szczecin, Poland; domi.maciejka@wp.pl (D.M.); ryterska.karina@gmail.com (K.R.); \\ majaczerwinska89@gmail.com (M.C.-R.); dominikajamiol@interia.pl (D.J.-M.); karzyd@pum.edu.pl (K.S.-Ż.) \\ 4 Liver and Internal Medicine Unit, Department of General, Transplant and Liver Surgery of the Medical \\ University of Warsaw, 02-097 Warsaw, Poland; p.milkiewicz@wp.pl (P.M.); jorasz@gmail.com (J.R.-W.) \\ 5 Translational Medicine Group, Pomeranian Medical University, 71-210 Szczecin, Poland \\ * Correspondence: ewa.stachowska@pum.edu.pl or ewast@pum.edu.pl
}

Received: 1 September 2018; Accepted: 10 November 2018; Published: 18 November 2018

\begin{abstract}
Introduction: Zonulin (ZO) has been proposed as a marker of intestinal permeability. Only a few studies have analyzed to date how diet influences the serum concentration of $\mathrm{ZO}$ among patients with non-alcoholic fatty liver disease (NAFLD). We performed a six-month dietetic intervention to evaluate the association between fiber intake and $\mathrm{ZO}$ concentration in 32 individuals with NAFLD. (2) Methods: Fiber content in the diet was estimated by Food Frequency Questionnaire (FFQ) and by analyzing 72-h nutritional diaries. ZO concentrations in serum were measured before and after the intervention by immunoenzymatic assay (ELISA). Fatty liver was quantified using the Hamaguchi score before and after the dietetic intervention. (3) Results: During the intervention, the dietary fiber intake increased from $19 \mathrm{~g} /$ day to the $29 \mathrm{~g} /$ day concomitant with an increase in the frequency of fiber consumption. All patients experienced significant (all $p<0.05$ ) improvements in serum aspartate aminotransferase (AST), alanine aminotransferase (ALT) and gamma-glutamyltransferase (GGTP) activities. We also detected decreased serum triglycerides $(p=0.036)$, homeostatic model assessment insulin resistance (HOMA-IR $(p=0.041)$ and insulin content $(p=0.34)$, and improvement of fatty liver status according to the Hamaguchi score $(p=0.009)$. $\mathrm{ZO}$ concentration in serum decreased by nearly $90 \%(7.335 \pm 13.492 \mathrm{vs} .0 .507 \pm 0.762 \mathrm{ng} / \mathrm{mL}$, $p=0.001)$ and correlated with the amount of dietary fiber intake $(p=0.043)$ as well as the degree of fatty liver $(p=0.037)$. (4) Conclusion: Increasing nutritional fiber results in reduced serum ZO levels, reduced liver enzymes and improved hepatic steatosis in patients with NAFLD, possibly by altering intestinal permeability. Increased dietary fiber intake should be recommended in patients with NAFLD.
\end{abstract}

Keywords: NAFLD; zonulin; fiber; diet

\section{Introduction}

The gut epithelium represents a key protective barrier separating internal organs from the adverse environment of the gut lumen [1]. Its permeability is controlled by several multiprotein adhesive 
complexes including tight junctions (TJ), subjacent adherens junctions (AJ), and desmosomes [1]. The intestinal barrier is a highly dynamic structure shaped by interactions with internal and external stimuli, such as cytokines, growth factors, and bacteria [1-3]. Diet-derived substances also interact with the barrier and modulate its permeability. For instance, glutamine [4,5] and tryptophan [6], peptides derived from cheese and milk [7,8], and polyphenols [9] act as positive regulators of epithelial permeability, whereas other compounds such as gliadin [10] or medium-chain fatty acids [11,12] negatively regulate intestinal barrier permeability. Zonulin (ZO) is a $47-\mathrm{kDa}$ protein that regulates intestinal permeability by modulating intracellular TJ structure and function [13]. The secretion of ZO into serum is believed to be regulated by several factors, including components of the diet $[14,15]$.

Nonalcoholic fatty liver disease (NAFLD) is now the most common cause of chronic liver disease. It is currently detected in over $20 \%$ of Europeans, and its prevalence is predicted to increase over the years $[16,17]$. NAFLD is considered the hepatic manifestation of the metabolic syndrome, and the steatotic phenotype appears to be worsened by disruptions in the host gut microbiota [18,19]. An increasing body of evidence demonstrates that NAFLD correlates positively with the phenomenon of "leaky gut", the mechanism encompassing TJ damage [20] in both young patients [13] and adults [21,22]. The disturbances of the gut-liver axis might explain, at least to a certain extent, the phenotype of fatty liver [23]. For example, gut dysbiosis and skewed production of inflammatory markers, among others lipopolysachcaride, might modulate the progression of NAFLD. This is underscored by the association between endotoxemia and severe forms of fatty liver [24]. Moreover, a dysbiotic gut ecosystem might result in increased production of endogenous ethanol, which could disrupt the integrity of the intestinal barrier and further increase liver injury in the setting of increased hepatic fat accumulation [25]. Several studies have reported that ZO concentrations increase significantly with the severity of steatosis $[13,20,21]$ and positively correlate with body mass index (BMI), liver histopathology, fasting insulin, and HOMA-IR as well as concentrations of inflammatory markers (e.g., serum IL-6) [21]. To the best of our knowledge, there is only one study that has described how a reduction diet among NAFLD patients might influence the serum concentration of $\mathrm{ZO}$ [13]. Here we present the impact of a six-month dietetic intervention, comprising an increased intake of fiber and a decreased intake of fat and simple sugars, on the liver status and ZO serum concentrations in a cohort of 32 patients with NAFLD.

\section{Materials and Methods}

\subsection{Study Design}

In the present study we included subjects selected from the Nutrient-Induced Insulin Output Ratio (NIOR) study, which was intended to investigate nutritional strategies for the individualized treatment of NAFLD [22]. In brief, this study was designed as a randomized controlled intervention trial to compare the influence of three nutrigenetic dietary strategies on fatty liver during a six-month intervention [26]. The exclusion criteria were: Diabetes mellitus (DMII); chronic and acute liver diseases other than NAFLD; high levels of physical activity ( $>3000 \mathrm{kcal} /$ week in leisure-time physical activity); changes in physical activity during the dietary intervention; use of statins; any condition that could limit the mobility of the participant; not being able to attend control visits; vegetarianism or a need for other special diets; excessive consumption of alcohol (more than $20 \mathrm{~g}$ in women and more than $30 \mathrm{~g}$ in men, per day); and other drug addiction. Compliance with the diet was evaluated at 3 control points (at 1, 2, and 6 months after commencement of the diet) (Figure 1). All subjects were instructed to maintain their usual physical activity (metabolic equivalent of the task; MET), which was controlled by a 2-h physical activity questionnaire. Initially, 171 Caucasian adults were enrolled in the NIOR study; however, 5 subjects did not meet the inclusion criteria. After the first month of the study further 29 individuals were excluded from the study: Either they did not comply with the prescribed diet, engaged in too much physical activity or they failed to complete the dietary intake questionnaire. 
At the second control meeting (i.e., 2 months after the beginning of the diet), 14 participants were excluded from the study for similar reasons. During the final visit (after 6 months), a further 13 participants were excluded. As a result, a total of 110 participants were adherent to the intervention protocol and remained in the study (Figure 1). We used an online randomization tool [26] to select patients for biochemical analyses. Serum samples from 32 participants (22 males and 10 females) were available for the current analysis. The fiber consumption was analyzed (soluble fiber as well as insoluble fiber) with the help of the Fineli base (https: / fineli.fi/fineli/en/index) and the Diet program (IZZ Poland). The study protocol was approved by the ethics committee of the Pomeranian Medical University and conformed to the ethical guidelines of the 1975 Declaration of Helsinki (Szczecin, Poland, 25012010 KB-0012/09/10). Participants provided written informed consent before the study.

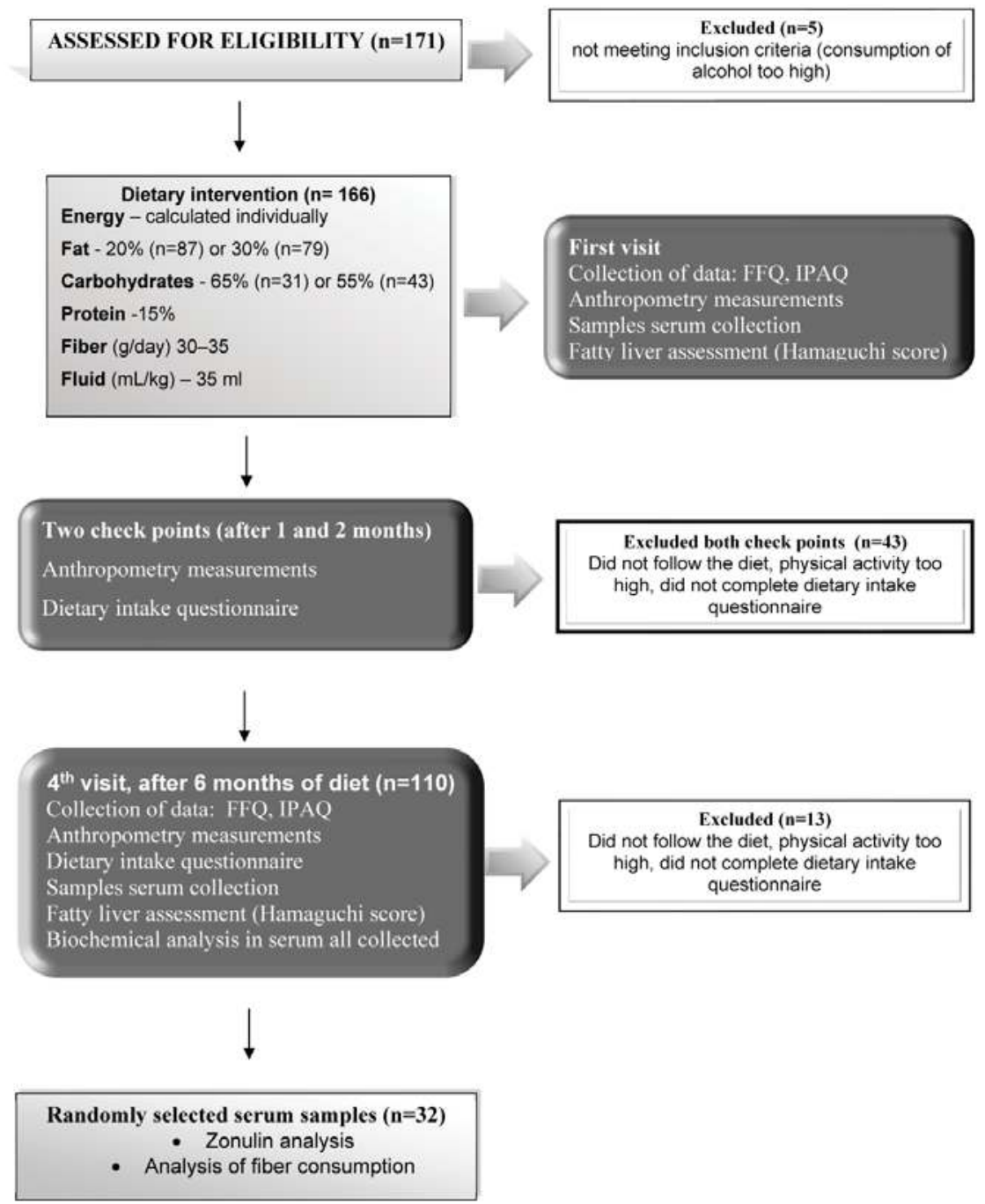

Figure 1. Flowchart for the inclusion of individuals in the study. 


\subsection{The Nutritional Intervention and Control}

The NIOR diet was modified on the basis of the of the American Heart Association (AHA) guidelines, including 3-5 meals/day, modified by the genetic result of NIOR as described in detail [27]. Menus were prepared as a daily plan for the 7 days of the week and included guidance on the daily timing of the five meals.

Nutrition patterns were analyzed with a Food Frequency Questionnaire (FFQ) and a 72-h food diary (including 2 working days and one day free of work) [27]. Fiber content (soluble and insoluble) was estimated by means of two methods: frequency of consumption by means of the FFQ and by analyzing the 72 nutritional diaries (from the three control points) via the Diet 5 program (IZZ Poland) and Fineli (the Finnish database). Dietary content, nutritional sources and the products used are presented in Table 1. Three portions of vegetables and two portions of fruit were introduced to the patients' diet. The recommended sources of insoluble fiber were as follows: Whole wheat bread, whole-wheat pasta, cereal and brown rice, whole rye bread, and graham bread. Recommended vegetables were: Tomatoes, savoy cabbages (fresh and pickled), napa cabbages, green beans, paprika, fresh and pickled cucumbers, onions, chives, red beans, lentils, spinach, carrots, leeks, celery, broccoli, lettuce, and dill. Recommended fruits were: apples, plums, and apricots (including dried). Finally, recommended seeds and nuts were: pumpkin seeds and walnuts. The level of physical activity was assessed using the questionnaire technique with validated International Physical Activity Questionnaire (IPAQ). Physical exercise was estimated with MET units (min/week). During the first and final visit, the degree of fatty liver was evaluated by a trained physician according to the Hamaguchi score [28], using a high-resolution B-mode abdominal ultrasound scanner (Acuson X300). Hamaguchi score $\geq 2.0$ was set as the cut-off for NAFLD [28].

Table 1. The nutritional products used in the diet.

\begin{tabular}{|c|c|c|}
\hline Variable & Recommendation & Recommended Sources \\
\hline Energy & \multicolumn{2}{|c|}{ Calculated Individually } \\
\hline Fiber (g/day) & $30-35$ & $\begin{array}{l}3 \text { portions of vegetables a day; } 2 \text { portions of } \\
\text { fruit as: fresh, fermented or boiled, wheat } \\
\text { bread, whole-wheat pasta, cereal and brown } \\
\text { rice }\end{array}$ \\
\hline Fat as percentage of total calories (\%) & $20-30$ & $\begin{array}{l}\text { Vegetable fats with a predominance of } \\
\text { rapeseed oil and olive oil. }\end{array}$ \\
\hline $\begin{array}{l}\text { Carbohydrates (low and medium IG), } \\
\text { percentage of total calories (\%) }\end{array}$ & $55-65$ & $\begin{array}{l}\text { Low and medium glycemic index as: wheat } \\
\text { and mix rye/wheat bread, whole-pasta, } \\
\text { cereal and brown rice, fruit and vegetables. }\end{array}$ \\
\hline $\begin{array}{c}\text { Simple carbohydrate, percentage of } \\
\text { total calories }(\%)\end{array}$ & $5-10$ & Dry fruits \\
\hline Protein $(\%)$ & 15 & $\begin{array}{l}\text { Poultry (chicken and turkey), fish (oily fish } 3 \\
\text { times a week), fermented dairy products ( } 2 \\
\text { times a day), eggs ( } 4-5 \text { a week), lean cottage } \\
\text { cheese, cheese with reduced fat content. }\end{array}$ \\
\hline Fluid (mL/kg) & 35 & $\begin{array}{c}\text { Water, coffee (1-2 cup a day) tea (black, } \\
\text { green). }\end{array}$ \\
\hline Vitamins and minerals & $\begin{array}{l}\text { Consistent with recommended } \\
\text { daily allowance }\end{array}$ & Natural sources from vegetables and fruit \\
\hline
\end{tabular}

\subsection{Laboratory Analyses}

Venous fasted blood samples were collected into tubes containing anticoagulant Ethylenediaminetetraacetic acid (EDTA). Blood samples were centrifuged at $3500 \mathrm{rpm}$ for $10 \mathrm{~min}$ at $4{ }^{\circ} \mathrm{C}$ within $2 \mathrm{~h}$ of collection. Standard blood biochemical analyses were carried out at the University Hospital Laboratory (Szczecin, Poland). Plasma ZO concentrations were measured by ELISA (Immundiagnostik AG, Bensheim, Germany) according to manufacturer's instructions. 


\subsection{Statistics}

For statistical analyses, R software 3.0.2 was used and all results were expressed as mean and standard deviation. Since the distribution in most cases deviated from normality (Shapiro-Wilk test), non-parametric paired tests were used (Wilcoxon signed-rank test) and $p<0.05$ was considered as statistically significant. The correlations between quantitative variables were calculated using the Spearman's correlation test.

\section{Results}

\subsection{The Amount of Zonulin in Serum Decreases with the Improvement of Fatty Liver}

As shown in Figure 2, the six-month dietetic intervention resulted in a significant improvement of liver injury markers in the studied group of 32 individuals as reflected by decreases of serum AST $(p=0.001)$, ALT $(p=0.001)$ and GGTP $(p=0.03)$. As shown in Table 2 , it also led to a reduction of the Hamaguchi score $(2.87 \pm 0.59$ vs. $1.40 \pm 0.93 ; p=0.009)$. Furthermore, serum triglyceride concentrations $(p=0.036)$, HOMA-IR $(p=0.041)$ index as well serum insulin $(p=0.34)$ decreased as a result of the dietetic intervention. Notably, we also detected a significant $(p=0.001)$, almost $90 \%$ reduction of serum ZO concentration as shown in Figure 2A. Table 3 demonstrates that serum ZO correlated with the degree of fatty liver (i.e., with Hamaguchi score $(p=0.037)$ serum AST $(p=0.041)$ and $\operatorname{ALT}(p=0.043)$ ), total lipids $(p=0.041)$, as well as low-density lipoprotein $(p=0.029)$. Interestingly, we detected a negative correlation between high-density lipoprotein and serum ZO contents (Table 3 ) but no significant link between ZO concentration and BMI or glucose metabolism parameters (all $p>0.05$ ).
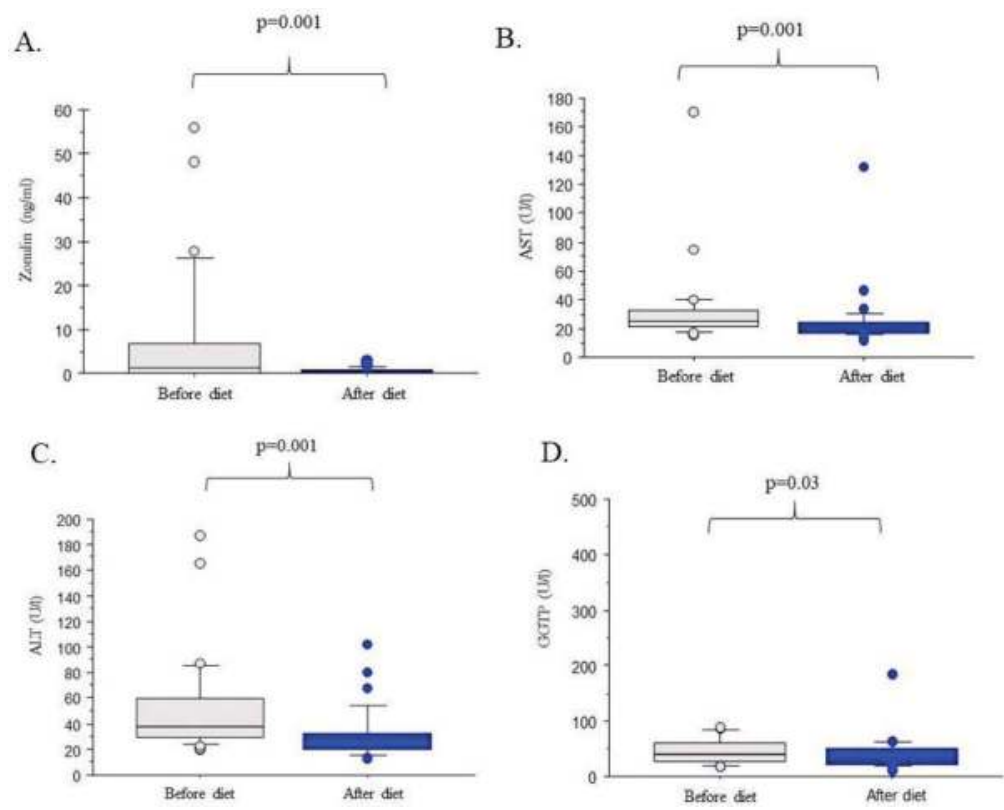

Figure 2. Zonulin concentration and liver function tests before and after the dietetic intervention. (A) Zonulin concentration. (B) Aspartate aminotransferase (AST) activity. (C) Alanine aminotransferase (ALT) activity. (D) Gamma-glutamyltransferase (GGTP) activity. 
Table 2. Baseline characteristics of the study cohort $(n=32)$, as well as effects of the dietetic intervention.

\begin{tabular}{cccc}
\hline Parameters & Before Diet & After Diet & $p$ Value \\
\hline Age (year) & $48.03 \pm 13.13$ & - & - \\
Body Mass $(\mathrm{kg})$ & $98.76 \pm 19.80$ & $91.64 \pm 16.60$ & $\mathrm{NS}$ \\
BMI $\left(\mathrm{kg} / \mathrm{m}^{2}\right)$ & $33.19 \pm 5.71$ & $30.91 \pm 5.45$ & $\mathrm{NS}$ \\
Fat mass $(\mathrm{kg})$ & $38.08 \pm 6.06$ & $32.84 \pm 9.41$ & $\mathrm{NS}$ \\
Lean body mass $(\mathrm{kg})$ & $60.93 \pm 12.90$ & $58.48 \pm 12.04$ & $\mathrm{NS}$ \\
Water content $(\mathrm{kg})$ & $45.25 \pm 9.16$ & $43.81 \pm 8.07$ & $\mathrm{NS}$ \\
Fatty liver (Hamaguchi score) & $2.87 \pm 0.60$ & $1.40 \pm 0.93$ & 0.009 \\
Triacylglycerols $(\mathrm{mg} / \mathrm{dL})$ & $219.09 \pm 326.01$ & $166.31 \pm 219.71$ & 0.036 \\
Total cholesterol (mg/dL) & $206.06 \pm 52.54$ & $200.56 \pm 45.44$ & $\mathrm{NS}$ \\
High density lipoprotein (mg/dL) & $44.84 \pm 10.09$ & $49.47 \pm 14.47$ & $\mathrm{NS}$ \\
Low density lipoprotein (mg/dL) & $121.15 \pm 33.43$ & $122 . \pm 60.32$ & $\mathrm{NS}$ \\
Total lipids (mg/dL) & $731.06 \pm 414.63$ & $706.44 \pm 300.43$ & $\mathrm{NS}$ \\
Glucose (mg/mL) & $104.50 \pm 20.01$ & $100.78 \pm 10.72$ & $\mathrm{NS}$ \\
Insulin (U/mL) & $17.90 \pm 11.44$ & $7.47 \pm 5.34$ & 0.034 \\
HOMA-IR & $4.85 \pm 3.14$ & $1.89 \pm 1.36$ & 0.041 \\
\hline
\end{tabular}

Abbreviation: BMI, body mass index; HOMA-IR, homeostasis model assessment of insulin resistance; NS, not significant.

Table 3. Correlations between serum zonulin content and patient characteristics.

\begin{tabular}{ccc}
\hline Parameters & RHO & $p$ Value \\
\hline Age (years) & 0.05 & $\mathrm{NS}$ \\
Body Mass $(\mathrm{kg})$ & $<0.01$ & $\mathrm{NS}$ \\
BMI $\left(\mathrm{kg} / \mathrm{m}^{2}\right)$ & $(-) 0.18$ & $\mathrm{NS}$ \\
Fat mass $(\mathrm{kg})$ & $(-) 0.13$ & $\mathrm{NS}$ \\
Fat content $(\%)$ & $(-) 0.17$ & $\mathrm{NS}$ \\
Lean body mass $(\mathrm{kg})$ & 0.09 & $\mathrm{NS}$ \\
Water content $(\mathrm{kg})$ & 0.13 & $\mathrm{NS}$ \\
Aspartate transaminase (U/L) & 0.35 & 0.041 \\
Alanine transaminase (U/L) & 0.36 & 0.043 \\
Gamma-glutamyltransferase (U/L) & 0.19 & $\mathrm{NS}$ \\
Triacylglycerols (mg/dL) & 0.17 & $\mathrm{NS}$ \\
Total cholesterol (mg/dL) & 0.22 & $\mathrm{NS}$ \\
High density lipoprotein (mg/dL) & $(-) 0.30$ & 0.029 \\
Low density lipoprotein (mg/dL) & 0.37 & 0.032 \\
Total Lipids $(\mathrm{mg} / \mathrm{dL})$ & 0.31 & 0.041 \\
Glucose (mg/mL) & $(-) 0.07$ & $\mathrm{NS}$ \\
Insulin (U/mL) & $(-) 0.10$ & $\mathrm{NS}$ \\
Fatty liver (Hamaguchi score) & 0.33 & 0.037 \\
\hline
\end{tabular}

\subsection{The Content of Zonulin in the Serum May be Related to the Increase in Fiber Consumption}

The analysis of nutritional diaries showed that all patients included in the analysis followed the suggested nutritional recommendations. No significant deviations from the proposed energy supply or key nutrients intake (proteins, carbohydrates and fats) were observed. Recruited patients consumed on average $29.24 \pm 10.97 \mathrm{~g}$ fiber per day, which is higher than the fiber intake as estimated for the Polish population i.e., $19 \mathrm{~g}$ per day [29]. This included: soluble fiber $(6.69 \pm 3.21 \mathrm{~g}$ per day) and insoluble fiber (17.44 $\pm 7.11 \mathrm{~g}$ per day). These calculations were done based on a 72-h recall diary subjected to the Fineli base. According to the database of the Institute of Nutrition in Poland (IZZ), the calculated fiber content was slightly lower: $28.18 \mathrm{~g}$ per day. We estimated that the frequency of consumption of fruits and vegetables rose among study participants from a few portions per week to a few portions per day (Table 4). The Spearman's rank analysis indicated the existence of a correlation between the amount of fiber in the diet and the concentration of ZO in blood ( $p=0.043)$ (Table 5). No other analyzed food constituent proved to correlate with serum ZO $(p>0.05)$. 
Table 4. Changes in patients dietary patterns during the intervention.

\begin{tabular}{cccc}
\hline Frequency of Consumption According FFQ & Before Intervention & During the Diet & Change in Frequency \\
\hline Dairy products & Every day & Every day & No change \\
Cereal products & A few times a day & A few times a day & No change \\
Fats & Every day & Every day & No change \\
Fruits & A few times a week & A few times a day & Increased \\
Vegetables and grains & A few times a week & A few times a day & Increased \\
Poultry, meet and fish & Every day & Every day & No change \\
\hline
\end{tabular}

Abbreviation: FFQ, food frequency questionnaire.

Table 5. Correlations between diet compounds and the concentration of serum zonulin.

\begin{tabular}{ccc}
\hline Parameters & RHO & $p$ Value \\
\hline Energy of diet (kcal) & 0.01 & NS \\
Protein (\%) & $(-) 0.01$ & NS \\
Fat (\%) & $(-) 0.07$ & NS \\
Saturated fatty acids (\%) & $(-) 0.02$ & NS \\
Monounsaturated fatty acids (\%) & $(-) 0.03$ & NS \\
Polyunsaturated fatty acids (\%) & $(-) 0.20$ & NS \\
Carbohydrates (\%) & 0.07 & NS \\
Fiber (\%) & $(-) 0.30$ & 0.043 \\
\hline
\end{tabular}

\section{Discussion}

NAFLD is turning into the most frequent liver condition worldwide, and diet is regarded as one of the major drivers of the steatotic phenotype. In our study we reconstructed the amount and frequency of dietary fiber consumption in patients with NAFLD and detected a correlation between $\mathrm{ZO}$ concentrations and fiber intake. Previously Pacifico et al. [13] demonstrated that serum ZO is elevated in the blood of obese children with NAFLD: ZO values correlated with steatosis, but not with fibrosis score, or the presence of nonalcoholic steatohepatitis (NASH). The results presented in the study by Hendy et al. [21] suggest that ZO levels could be used for a quick and noninvasive diagnosis of liver inflammation. It was observed that once ZO serum concentration in a patient with NAFLD exceeds $8.3 \mathrm{ng} / \mathrm{mL}$, the risk of developing NASH rises considerably. This might be significant for the development of non-invasive biomarker panels that could substitute liver biopsy [21].

In our study, we examined whether $\mathrm{ZO}$ concentrations decreased during the introduction of calorie restriction. Damms-Machado et al. [30] evaluated ZO contents in the stool of patients who underwent a 12-month dietary intervention and showed that $\mathrm{ZO}$ concentrations in stool are not influenced by weight reduction therapy and do not correlate with anthropometric variables. In our study we did not detect any significant correlations between serum $\mathrm{ZO}$ concentrations and the reduction of body mass parameters either. Similarly to Damms-Machado et al. [30], we observed a correlation between ZO and lipid parameters, enzymatic markers of liver injury and the degree of steatosis. Damms-Machado et al. observed that $\mathrm{ZO}$ concentrations among patients did not change significantly with steatosis over the course of the study [30]. They concluded that the lack of fecal ZO alterations after the intervention was a result of ZO-independent regulation of TJ occurring during the course of weight reduction, which leads to the differences in gut permeability. We previously described a similar phenomenon (ZO-independent regulation) in a study in which we analyzed the permeability of the barrier after the use of colostrum [15].

We speculate that changes in fiber intake were responsible for the reduction of $\mathrm{ZO}$ in the blood of our patients. Indeed, the level of dietary fiber rose in the diet of our patients regardless of the supply of other nutrients (i.e., fat or carbohydrates). Our patients were subjected to control visits four times during the 6-month dietary intervention, and only those patients who significantly increased their frequency of consumption and supply of soluble and insoluble fiber completed the study. A recently published study found that fruit fiber consumption was associated with improved liver health [31]. According to these results it was postulated that higher insoluble fiber consumption ( $\geq 7.5 \mathrm{~g} /$ day) 
leads to improvements in fatty liver parameters. Notably, a regression model proved a relationship between liver status and fruit derived fiber [31]. Our study has some limitations that should be kept in mind when interpreting the data. First of all, included patients were not biopsied, hence we did not have data on the presence of NASH. For the same reason, we did not have data on the degree of liver fibrosis in our patients. We focused on the measurements of zonulin, however other markers of intestinal permeability such as bacterial endotoxin levels, LBP protein concentration or serum citrulin might also provide additional insights into observed changes during diet. Finally, the studied cohort was relatively small and the Hamaguchi score [28], which we applied to quantify NAFLD, has not been thoroughly validated in Caucasians.

Why might the above results be of significance? Fibers fermenting in the intestine provide short-chain fatty acids that fulfill a protective and nourishing role for colonocytes, ensuring the preservation of the intestinal barrier [32]. Plant fiber provides nourishment for intestinal microbiota, which are of prime importance to the preservation of the intestinal barrier integrity [31]. The results of our study imply that appropriate fiber intake helps to maintain the proper structure and function of the intestinal barrier. The lifestyle recommendations for successful management of NAFLD promote fruit and vegetables in the diet of NAFLD patients. As our results show, the increased supply of fiber positively influences the NAFLD-associated parameters and it should be promoted among the specialists designing the diets for patients with fatty liver.

Author Contributions: Conceptualization, E.S., P.M. and M.K.; Methodology E.S., J.R.W.; Software, D.M.; Validation, K.R.; Formal Analysis, M.K., E.S., P.M.; Investigation D.J.M., M.C.R., J.R.; Resources, K.R., E.S.; Data Curation, K.R.; Writing-Original Draft Preparation, E.S., Writing-Review \& Editing, M.K.; Visualization, D.M.; Supervision, P.M.; Project Administration, K.R.; Funding Acquisition, E.S.

Funding: This research was funded by NCN, No. N404 150539.

Acknowledgments: We are grateful to Małgorzta Standowicz, Anna Sabinicz, Małgorzata Napierała for their dietary supervision as well as to Roman Liebe for the proofreading of the manuscript.

Conflicts of Interest: The authors declare no conflict of interest.

\section{References}

1. Lechuga, S.; Ivanov, A.I. Disruption of the epithelial barrier during intestinal inflammation: Quest for new molecules and mechanisms. Biochim. Biophys. Acta 2017, 7, 1183-1194. [CrossRef] [PubMed]

2. Choi, W.; Yeruva, S.; Turner, J.R. Contributions of intestinal epithelial barriers to health and disease. Exp. Cell Res. 2017, 358, 71-77. [CrossRef] [PubMed]

3. Shimizu, M. Interaction between food substances and the intestinal epithelium. Biosci. Biotechnol. Biochem. 2010, 74, 232-241. [CrossRef] [PubMed]

4. Peng, X.; Yan, H.; You, Z.; Wang, P.; Wang, S. Effects of enteral supplementation with glutamine granules on intestinal mucosal barrier function in severe burned patients. Burns 2004, 30, 135-139. [CrossRef] [PubMed]

5. Ding, L.A.; Li, J.S. Effects of glutamine on intestinal permeability and bacterial translocation in TPN-rats with endotoxemia. World J. Gastroenterol. 2003, 9, 1327-1332. [CrossRef] [PubMed]

6. Watanabe, J.; Fukumoto, K.; Fukushi, E.; Sonoyama, K.; Kawabata, J. Isolation of tryptophan as an inhibitor of ovalbumin permeation and analysis of its suppressive effect on oral sensitization. Biosci. Biotechnol. Biochem. 2004, 68, 59-65. [CrossRef] [PubMed]

7. Yasumatsu, H.; Tanabe, S. The casein peptide Asn-Pro-Trp-Asp-Gln enforces the intestinal tight junction partly by increasing occludin expression in Caco-2 cells. Br. J. Nutr. 2010, 104, 951-956. [CrossRef] [PubMed]

8. Hashimoto, K.; Nakayama, T.; Shimizu, M. Effects of beta-lactoglobulin on the tight-junctional stability of Caco-2-SF monolayer. Biosci. Biotechnol. Biochem. 1998, 62, 1819-1821. [CrossRef] [PubMed]

9. Suzuki, T. Regulation of intestinal epithelial permeability by tight junctions. Cell. Mol. Life Sci. 2013, 70, 631-659. [CrossRef] [PubMed]

10. Hollon, J.; Puppa, E.L.; Greenwald, B.; Goldberg, E.; Guerrerio, A.; Fasano, A. Effect of gliadin on permeability of intestinal biopsy explants from celiac disease patients and patients with non-celiac gluten sensitivity. Nutrients 2015, 7, 1565-1576. [CrossRef] [PubMed] 
11. Usami, M.; Muraki, K.; Iwamoto, M.; Ohata, A.; Matsushita, E.; Miki, A. Effect of eicosapentaenoic acid (EPA) on tight junction permeability in intestinal monolayer cells. Clin. Nutr. 2001, 20,351-359. [CrossRef] [PubMed]

12. Usami, M.L.; Komurasaki, T.; Hanada, A.; Ohata, A.; Matsushita, E.; Miki, A. Effect of gamma-linolenic acid or docosahexaenoic acid on tight junction permeability in intestinal monolayer cells and their mechanism by protein kinase C activation and/or eicosanoid formation. Nutrition 2003, 19, 150-166. [CrossRef]

13. Pacifico, L.; Bonci, E.; Marandola, L.; Romaggioli, S.; Bascetta, S.; Chiesa, C. Increased circulating zonulin in children with biopsy-proven nonalcoholic fatty liver disease. World J. Gastroenterol. 2014, 20, 17107-17114. [CrossRef] [PubMed]

14. Fasano, A. Zonulin, regulation of tight junctions, and autoimmune diseases. Ann. N. Y. Acad. Sci. 2012, 1258, 25-33. [CrossRef] [PubMed]

15. Hałasa, M.; Maciejewska, D.; Baśkiewicz-Hałasa, M.; Machaliński, B.; Safranow, K.; Stachowska, E. Oral Supplementation with Bovine Colostrum Decreases Intestinal Permeability and Stool Concentrations of Zonulin in Athletes. Nutrients 2017, 9, e370. [CrossRef] [PubMed]

16. European Association for the Study of the Liver (EASL); European Association for the Study of Diabetes (EASD); European Association for the Study of Obesity (EASO). EASL-EASD-EASO Clinical Practice Guidelines for the management of non-alcoholic fatty liver disease. J. Hepatol. 2016, 64, 1388-1402. [CrossRef] [PubMed]

17. Estes, C.; Anstee, Q.M.; Arias-Loste, M.T.; Bantel, H.; Bellentani, S.; Caballeria, J.; Colombo, M.; Craxi, A.; Crespo, J.; Day, C.P.; et al. Modeling NAFLD Disease Burden in China, France, Germany, Italy, Japan, Spain, United Kingdom, and United States for the period 2016-2030. J. Hepatol 2018, in press. [CrossRef] [PubMed]

18. Henao-Mejia, J.; Elinav, E.; Jin, C.; Hao, L.; Mehal, W.Z.; Strowig, T.; Thaiss, C.A.; Kau, A.L.; Eisenbarth, S.C.; Jurczak, M.J.; et al. Inflammasome-mediated dysbiosis regulates progression of NAFLD and obesity. Nature 2012, 482, 179-185. [CrossRef] [PubMed]

19. Shen, F.; Zheng, R.D.; Sun, X.Q.; Ding, W.J.; Wang, X.Y.; Fan, J.G. Gut microbiota dysbiosis in patients with non-alcoholic fatty liver disease. Hepatobiliary Pancreat. Dis. Int. 2017, 16, 375-381. [CrossRef]

20. Miele, L.; Marrone, G.; Lauritano, C.; Cefalo, C.; Gasbarrini, A.; Day, C.; Grieco, A. Gut-liver axis and microbiota in NAFLD: Insight pathophysiology for novel therapeutic target. Curr. Pharm. Des. 2013, 19, 5314-5324. [CrossRef] [PubMed]

21. Hendy, O.M.; Elsabaawy, M.M.; Aref, M.M.; Khalaf, F.M.; Oda, A.M.A.; El Shazly, H.M. Evaluation of circulating zonulin as a potential marker in the pathogenesis of nonalcoholic fatty liver disease. Metabolism 2017, 69, 43-50. [CrossRef] [PubMed]

22. Wybranska, I.; Malczewska-Malec, M.; Partyka, L.; Kiec-Wilk, B.; Kosno, K.; Leszczynska-Golabek, I.; Zdzienicka, A.; Gruca, A.; Kwasniak, M.; Dembinska-Kiec, A. Evaluation of genetic predisposition to insulin resistance by nutrient-induced insulin output ratio (NIOR). Clin. Chem. Lab. Med. 2007, 45, 1124-1132. [CrossRef] [PubMed]

23. Poeta, M.; Pierri, L.; Vajro, P. Gut-Liver Axis Derangement in Non-Alcoholic Fatty Liver Disease. Children 2017, 4, 66. [CrossRef] [PubMed]

24. Pang, J.; Xu, W.; Zhang, X.; Wong, G.H.; Chan, A.H.; Chan, H.Y.; Tse, C.H.; Shu, S.T.; Choi, P.L.; Chan, H.Y.; et al. Significant positive association of endotoxemia with histological severity in 237 patients with non-alcoholic fatty liver disease. Aliment. Pharmacol. Ther. 2017, 46, 175-182. [CrossRef] [PubMed]

25. Engstler, A.J.; Aumiller, T.; Degen, C.; Dürr, M.; Weiss, E.; Maier, I.B.; Schattenberg, J.M.; Jin, C.J.; Sellmann, C.; Bergheim, I. Insulin resistance alters hepatic ethanol metabolism: Studies in mice and children with non-alcoholic fatty liver disease. Gut 2016, 65, 1564-1571. [CrossRef] [PubMed]

26. QuickCalcs. Available online: https://www.graphpad.com/quickcalcs/randomselect2 (accessed on 2 November 2016).

27. Stachowska, E.; Ryterska, K.; Maciejewska, D.; Banaszczak, M.; Milkiewicz, P.; Milkiewicz, M.; Gutowska, I.; Ossowski, P.; Kaczorowska, M.; Jamioł-Milc, D.; et al. Nutritional Strategies for the Individualized Treatment of Non-Alcoholic Fatty Liver Disease (NAFLD) Based on the Nutrient-Induced Insulin Output Ratio (NIOR). Int. J. Mol. Sci. 2016, 17, 1172. [CrossRef] [PubMed] 
28. Hamaguchi, M.; Kojima, T.; Itoh, Y.; Harano, Y.; Fujii, K.; Nakajima, T.; Kato, T.; Takeda, N.; Okuda, J.; Ida, K.; et al. The severity of ultrasonographic findings in nonalcoholic fatty liver disease reflects the metabolic syndrome and visceral fat accumulation. Am. J. Gastroenterol. 2007, 102, 2708-2715. [CrossRef] [PubMed]

29. Laskowski, W. Wartość Odżywcza Diety Polaków Oraz jej Zmiany; Współczesne Kierunki Działań Prozdrowotnych, red. A.; Wolska-Adamczyk, WSIiZ: Warszawa, Poland, 2015.

30. Damms-Machado, A.; Louis, S.; Schnitzerm, A.; Volynets, V.; Rings, A.; Basrai, M. Gut permeability is related to body weight, fatty liver disease, and insulin resistance in obese individuals undergoing weight reduction. Am. J. Clin. Nutr. 2017, 105, 127-135. [CrossRef] [PubMed]

31. Cantero, I.; Abete, I.; Monreal, J.I.; Martinez, J.A.; Zulet, M.A. Fruit Fiber Consumption Specifically Improves Liver Health Status in Obese Subjects under Energy Restriction. Nutrients 2017, 9, e667. [CrossRef] [PubMed]

32. Rinella, M.E.; Sanyal, A.J. Management of NAFLD: A stage-based approach. Nat. Rev. Gastroenterol. Hepatol. 2016, 13, 196-205. [CrossRef] [PubMed]

(C) 2018 by the authors. Licensee MDPI, Basel, Switzerland. This article is an open access article distributed under the terms and conditions of the Creative Commons Attribution (CC BY) license (http:/ / creativecommons.org/licenses/by/4.0/). 
MDPI

St. Alban-Anlage 66

4052 Basel

Switzerland

Tel. +41616837734

Fax +41 613028918

www.mdpi.com

Nutrients Editorial Office

E-mail: nutrients@mdpi.com

www.mdpi.com/journal/nutrients

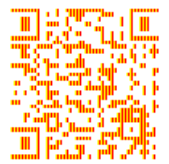



MDPI

St. Alban-Anlage 66

4052 Basel

Switzerland

Tel: +41 616837734

Fax: +41 613028918

www.mdpi.com 